MIT/WHOI

Massachusetts Institute of Technology Woods Hole Oceanographic Institution

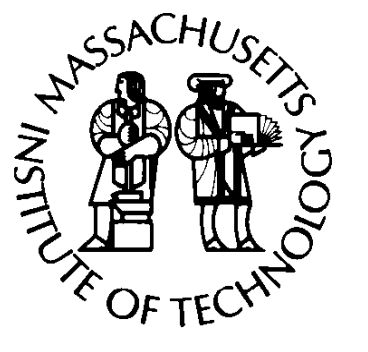

\author{
Joint Program \\ in Oceanographyl \\ Applied Ocean Science \\ and Engineering
}

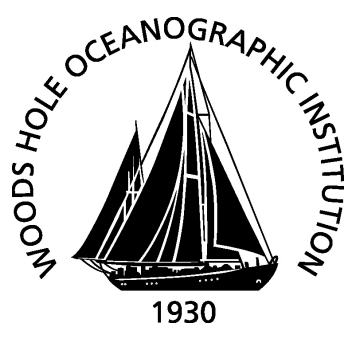

\title{
DOCTORAL DISSERTATION
}

Computational analysis of the biophysical controls on Southern Ocean phytoplankton ecosystem dynamics

Tyler W. Rohr

February 2019 


\title{
COMPUTATIONAL ANALYSIS OF THE BIOPHYSICAL CONTROLS ON SOUTHERN OCEAN PHYTOPLANKTON ECOSYSTEM DYNAMICS
}

\author{
by \\ Tyler W. Rohr \\ B.S.E., Duke University (2012) \\ Submitted in partial fulfillment of the requirements for the degree of \\ Doctor of Philosoplyy \\ at the \\ MASSACHUSETTS INSTITUTE OF TECHNOLOGY \\ and the \\ WOODS HOLE OCEANOGRAPHIC INSTITUTION
}

February 2019

C)2019 Tyler W. Rohr. All rights reserved.

The author hereby grants to MIT and WHOI permission to reproduce and to distribute publicly paper and electronic copies of this thesis document in whole or in part in any medium now known or hereafter created.

Author .

foint Program in Oceanography/Applied Ocean Science \& Engineering Massacluusetts Institute of Technology \& Woods Hole Oceanographic Institution December 19, 2018

Certified by

Scott Doney Joe D. and Helen J. Kington Professor in Environmental Change University of Virginia Thesis Supervisor

Certified by

David Nicholson Associate Scientist

Woods Hole Oceanographic Institution Thesis Supervisor

Accepted by .-

Shuhei Ono

Chair, Joint Committee for Chemical Oceanography

Massachusetts Institute of Technology

Woods Hole Ocemographic Institution 


\title{
COMPUTATIONAL ANALYSIS OF THE BIOPHYSICAL CONTROLS ON SOUTHERN OCEAN PHYTOPLANKTON ECOSYSTEM DYNAMICS by \\ Tyler W. Rohr
}

\begin{abstract}
Submitted to the MIT-WHOI Joint Program in Oceanography and Applied Ocean Science and Engineering on December 19, 2018, in partial fulfillment of the requirements for the degree of Doctor of Philosophy in Chemical Oceanography
\end{abstract}

\begin{abstract}
Southern Ocean net community productivity plays an out sized role in regulating global biogeochemical cycling and climate dynamics. The structure of spatial-temporal variability in phytoplankton ecosystem dynamics is largely governed by physical processes but a variety of competing pathways complicate our understanding of how exactly they drive net population growth. Here, I leverage two coupled, 3-dimensional, global, numerical simulations in conjunction with remote sensing data and past observations, to improve our mechanistic understanding of how physical processes drive biology in the Southern Ocean. In Chapter 2, I show how different mechanistic pathways can control population dynamics from the bottom-up (via light, nutrients), as well as the top-down (via grazing pressure). In Chapters 3 and 4, I employ a higher resolution, eddy resolving, integration to explicitly track and examine closed eddy structures and address how they modify biomass at the mesoscale. Chapter 3 considers how simulated eddies drive bottom-up controls on phytoplankton growth and finds that division rates are, on average, amplified in anticyclones and suppressed in cyclones. Anomalous division rates are predominately fueled by an anomalous vertical iron flux driven by eddy-induced Ekman Pumping. Chapter 4 goes on to describe how anomalous division rates combine with anomalous loss rates to drive anomalous net population growth. Biological rate-based mechanisms are then compared to the potential for anomalies to evolve strictly via physical transport (i.e. dilution, stirring, advection). All together, I identify and describe dramatic regional and seasonal variability in when, where, and how different mechanisms drive phytoplankton growth throughout the Southern Ocean. Better understanding this variability has broad implications to our understanding of how oceanic biogeochemisty will respond to, and likely feedback into, a changing climate. Specifically, the uncertainty associated with this variability should temper recent proposals to artificially stimulate net primary production and the biological pump via iron fertilization. In Chapter $5 \mathrm{I}$ argue that Southern Ocean Iron Fertilization fails to meet the basic tenets required for adoption into any regulatory market based framework.
\end{abstract}

Thesis Supervisor: Scott Doney

Title: Joe D. and Helen J. Kington Professor in Environmental Change

University of Virginia

Thesis Supervisor: David Nicholson

Title: Associate Scientist

Woods Hole Oceanographic Institution 
Dedicated to my father, Dr. James Rohr 


\section{ACKNOWLEDGMENTS}

Like all things, an end and a beginning, but now also a sense of time and life catching up with one another. These sort of milestones don't come often, and they certainly don't come alone.

Scott, your gentle wisdom and unconditional support have guided me the whole way through. In science, your counsel has always come with a seed of insight and the space to let it grow, and in life, you have been endlessly accommodating to my strange geography. Matt, you consistently provided me with the resources, advice, and collaboration to forge ahead. Hugh, amongst other things, you took me to the end of the world and back. I will never forget how the soft Antarctic dusk would slip seamlessly into the somehow softer light of dawn. It was so beautiful that it barely seemed fair. Steph, you have been kind and insightful throughout, and Roo, you graciously stepped up when I needed someone to co-advise me and you never missed a beat. Finally Cheryl, you more or less adopted me and I couldn't be more fortunate for it. I never enjoy talking about science more than when I'm talking with you. Thank you all. It is both trite and true to say I could not have done it without you.

To all my friends, from $T I$ to $G C$ to $J P$ to $L J$, thank you for always being there to indulge my odd fascinations and laugh the ghosts away. You blur the line between community and communion and reiterate the ancient truth that church is really just where ever you find it. That sort of spiritual clarity is a strong elixir for long days or hard years.

Recently, I've begun to find more and more of my church in the mountains. More than anywhere else, the mountains teach me how to feel happy and scarred at the same time. Measured against the hardly justifiable pangs of well-financed millennial anxiety, it can be a quiet miracle to feel brave without wondering if I deserve to. So, it is with immense gratitude and my whole heart when I thank everyone who has ever had the mind to tie in with me. It is a bond that is not soon forgotten. Where ever we have been - in the clouds on Pico Cão Grande, in the stars on Astroman, below the threat of storms on the Diamond, or above the flight of condors in Patagonia - thank you all for trusting me, and letting me trust you.

It may seem strange, but I would be remiss to not acknowledge the music that has accompanied, and at times defined, some of the most important pieces of my last half decade. How could I forget listening to Sunday Candy [the Rapper, C., 2016] while cramming with the JP Chem cohort to keep from going mad, then privately blasting Never Quite Free [Darnielle, J., 2016] in the moments where I thought maybe I had. In Antarctica, and then Patagonia, it was Jewels, R. T. [2016], Byrne, J. [2016], and XX, T. [2016] shepherding me through my great Southern Adventure. Last year, it was Ocean, F. [2016], Elverum, P. [2001, 2017] and Wrens, T. [2003], who kept me afloat in the moments that felt almost impossible. Everywhere else in between, from the crowded belly of Great Scott (e.g. Planet, D., 2015; Fathers, Y., 2016), to the backroom of a Reykjavik hostel [Baker, J., 2016], to the rain-soaked festival grounds (e.g. House, B. [2016]; Vernon, J. [2017]), to the old-grove cradle of California [Lekman, J., 2018], a steady drip of live music has brought me joy and shown me grace. And now, as I write this, and ponder the waning hours of a weight being lifting, What It's Like [Russel, A, $<1992)$ ] plays on the stereo and I think about just that.

And of course, my parents. Mom, you are fearless and strong and have inspired me more than you know. Watching you tack carefully between an impassioned engineer and a gypsy daydream reminds me how to split the difference between what I wish and what I want. 
Whether you like or not, the wild in you weaves through the wild in me and I wouldn't have it any other way.

And Dad, you taught me how to be proud of this crazy life. Sure, nobody gets it right, but we're all supposed to try, and that can be enough to exercise the things that haunt the hallways of wasted time. You always insisted that whatever I do, I do it with conviction, and then, at the end, you showed me how. For months you cast incantations with a subversive smile against our fears, refusing to give traction to the shape of things to come. Even deep in the trenches, with a head full of tumors, you kept it together. You kept laughing, kept teaching, kept loving, and most spectacularly kept yourself. Before you left, you explained in hopeful gasps that the aperture keeps getting smaller, but the focus stays the same.

This much, at least, is clear: I miss you, I love you, I am from you, and this thesis is, obviously, for you.

The CESM project is supported by the National Science Foundation and the Office of Science (BER) of the U.S. Department of Energy. Computing resources were provided by the Climate Simulation Laboratory at NCAR's Computational and Information Systems Laboratory (CISL), sponsored by the National Science Foundation and other agencies. This research was enabled by CISL compute and storage resources. TR was supported by an NDSEG graduate fellowship. TR and SCD acknowledge support from the National Aeronautics and Space Administration Ocean Biology and Biogeochemistry Program (NNX14AL86G). TR, SCD and MTK acknowledge support from the National Science Foundation Polar Programs award 1440435 (Antarctic Integrated System Science) to the Palmer LTER program. Please contact trohr@mit.edu for further questions or to access to data. 


\section{Contents}

1 Introduction $\quad 17$

1.1 Motivation . . . . . . . . . . . . . . . . . . . . 18

1.1.1 Marine primary production, the carbon cycle, and phytoplankton ecosystem dynamics . . . . . . . . . . . . . . . . . . . 18

1.1.2 The Southern Ocean . . . . . . . . . . . . . . . . . . . . . . 19

1.2 Mechanistic drivers of net population growth and bloom phenology . . . . . . . 20

1.2.1 Mixed layer depth . . . . . . . . . . . . . . . . . 20

1.2 .2 Eddies . . . . . . . . . . . . . . . . . . . . 21

1.2 .3 Sea Ice . . . . . . . . . . . . . . . . . . . . . . . . 22

1.2.4 Human intervention and Ocean Iron Fertilization . . . . . . . . . . . . . 22

1.3 Observational challenges and the role for models . . . . . . . . . . . . . . 23

1.4 Thesis Overview . . . . . . . . . . . . . . . . . . . . 24

2 Variability in the mechanisms controlling Southern Ocean phytoplankton bloom phenology in an ocean model and satellite observations $\quad 27$

2.1 Introduction . . . . . . . . . . . . . . . . . . . . . . . . . . 28

2.2 Methods . . . . . . . . . . . . . . . . . . . . . . . 31

2.2 .1 Numerical Experiments . . . . . . . . . . . . . . . . . . . . 31

2.2.2 Remote sensing and reanalysis data . . . . . . . . . . . . . . . . . 33

2.2.3 Quantification of relevant rates and metrics . . . . . . . . . . . . . 33

2.2.4 Model Skill Metrics . . . . . . . . . . . . . . . . . . . . . . . 36

2.2 .5 Regional Bins . . . . . . . . . . . . . . . . . . . 36

2.3 Results . . . . . . . . . . . . . . . . . . . . . 37

2.3.1 Large-scale Southern Ocean patterns . . . . . . . . . . . . . . . . . . 37

2.3.2 CESM Regional Case Studies: Zonal and Latitudinal Comparisons . . . 38

2.3.3 Remote Sensing/Reanalysis Zonal Comparison _ . . . . . . . . . . . . . 40

2.4 Discussion . . . . . . . . . . . . . . . . . . . . 41

2.5 Conclusions . . . . . . . . . . . . . . . . . . . . 45 
3 Simulated eddy induced bottom-up controls on phytoplankton growth in $\begin{array}{ll}\text { the Southern Ocean } & 57\end{array}$

3.1 Introduction . . . . . . . . . . . . . . . . . . . . . . . 59

3.2 Methods . . . . . . . . . . . . . . . . . . . . . . 61

3.2 .1 Numerical simulation . . . . . . . . . . . . . . . . . . . . . 61

3.2.2 Description of diagnostic variables . . . . . . . . . . . . 64

3.2.3 Depth extrapolation . . . . . . . . . . . . . . . . . 66

3.2.4 Anomaly and Climatology Fields . . . . . . . . . . . . . . . . 67

3.2.5 Eddy identification and tracking . . . . . . . . . . . . . 67

3.2 .6 Eddy Subsets . . . . . . . . . . . . . . . . . . . . . . . . . 69

3.3 Results . . . . . . . . . . . . . . . . . . . . . . 70

3.3.1 Simulated and observed eddy track demographics . . . . . . . . . . . . 70

3.3 .2 Mixing and light limitation . . . . . . . . . . . . . . . . . 71

3.3.3 Iron availability, limitation, and sources . . . . . . . . . . . . . 72

3.3 .4 Anomalous division rates . . . . . . . . . . . . . . . . . 74

3.3.5 Seasonal variability in deep mixing ACC eddies . . . . . . . . . . . . . 75

3.4 Discussion . . . . . . . . . . . . . . . . . . . . . . . . . 75

3.4.1 Comparison of theoretical, simulated and observed mixed layer depth

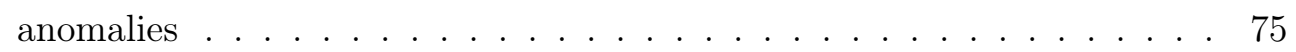

3.4.2 Comparison of theoretical, simulated and observed iron transport . . . . 77

3.4.3 Comparison of theoretical, simulated and observed anomalous division rates . . . . . . . . . . . . . . . . . . . . 78

3.4 .4 Basin scale iron budget f . . . . . . . . . . . . . . . . 79

3.5 Conclusions . . . . . . . . . . . . . . . . . . . . . . 81

3.6 Acknowledgments . . . . . . . . . . . . . . . . . . . . . . . . . . . 81

4 The simulated biological response to Southern Ocean eddies via biological rate modification and physical transport $\quad 95$

4.1 Introduction . . . . . . . . . . . . . . . . . . 97

4.2 Methods . . . . . . . . . . . . . . . . . . . . . 100

4.2 .1 Numerical Simulation . . . . . . . . . . . . . . . . . . . . 100

4.2 .2 Description of Diagnostic Tracers . . . . . . . . . . . . . . . . . . 102

4.2 .3 Depth Extrapolation . . . . . . . . . . . . . . . 105

4.2.4 Anomaly and climatology Fields . . . . . . . . . . . . . . . . 105

4.2.5 Eddy identification and tracking . . . . . . . . . . . . . . . . 105

4.2 .6 Eddy Subsets . . . . . . . . . . . . . . . . . . . 106

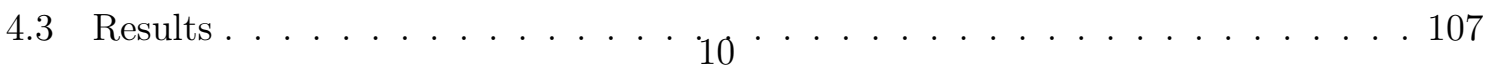


4.3.1 Rate-based mechanisms . . . . . . . . . . . . . . . . 107

4.3.2 Physical transport mechanisms . . . . . . . . . . . . . . . 109

4.3.3 Spatial and seasonal variability in biomass anomalies . . . . . . . . . . 110

4.3.4 Depth resolved seasonal variability . . . . . . . . . . . . . . . . 111

4.4 Discussion . . . . . . . . . . . . . . . . . . . . . . . . . 113

4.4.1 Seasonal variability in relative contribution from various mechanisms . . 113

4.4.2 Comparison of simulated and observed basin scale biomass distributions 116

4.4 .3 Notes on export . . . . . . . . . . . . . . . . . . . . . . 118

4.5 Conclusions . . . . . . . . . . . . . . . . . . . . . . . . . . . 119

4.6 Acknowledgments . . . . . . . . . . . . . . . . . . . . . 120

5 Southern Ocean Iron Fertilization: An argument against commercialization but for continued research amidst lingering uncertainty. 133

5.1 Introduction/Background . . . . . . . . . . . . . . . . . . . . . 134

5.2 The case against commercialization . . . . . . . . . . . . . . 136

5.2 .1 Will it work? . . . . . . . . . . . . . . . . . 137

5.2 .2 Auditing: Can it be measured? . . . . . . . . . . . . . . . . . 141

5.2.3 Safety: Will it have adverse side effects? . . . . . . . . . . . . . . . . . . 142

5.2.4 Managing uncertainty and market failures . . . . . . . . . . . . . . 145

5.3 The case for continued research . . . . . . . . . . . . . . . . 146

5.3.1 Prospects for commercialization on voluntary offset markets . . . . . . . 146

5.4 Conclusions . . . . . . . . . . . . . . . . . . . . . . . 150

6 Conclusion $\quad 155$

6.1 General results . . . . . . . . . . . . . . . . . . 156

6.2 Comments on modelling limitations . . . . . . . . . . . . . . . . . 157

6.2.1 Phytoplankton scheme . . . . . . . . . . . . . . . 157

6.2 .2 Zooplankton scheme . . . . . . . . . . . . . . . . 159

6.2 .3 Sea-ice biogeochemistry . . . . . . . . . . . . . . . . . 161

6.3 Moving forward . . . . . . . . . . . . . . . . . 161

A CESM surface concentration correction model 165

A.1 Introduction . . . . . . . . . . . . . . . . . 166

A.2 CESM surface concentration correction factor . . . . . . . . . . 166

A.3 Correction factor model . . . . . . . . . . . . . . . . . 166

A.4 Test of model skill . . . . . . . . . . . . . . . . . . . . . 167 
B Supplemental seasonal distributions of climatologies and eddy anomalies from Chapter 3

C Supplemental seasonal distributions of climatologies and eddy anomalies from Chapter 4

D References 


\section{List OF FigURES}

2-1 Peak bloom size and timing . . . . . . . . . . . . . . . . . 47

2-2 Relative size and timing of simulated depth-integrated phytoplankton specific growth rates . . . . . . . . . . . . . . . . . . . . . . 48

2-3 Regionally averaged, seasonal climatologies simulated by CESM; A1 and P1 . . 49

2-4 Simulated seasonal climatologies of phytoplankton rate and limitation terms; Bin $\mathrm{P} 1$ and $\mathrm{A} 1 \ldots \ldots \ldots \ldots$

2-5 Regionally averaged, seasonal climatologies simulated by CESM; P1, P2, and P3 51

2-6 Simulated seasonal climatologies of phytoplankton rate and limitation terms;

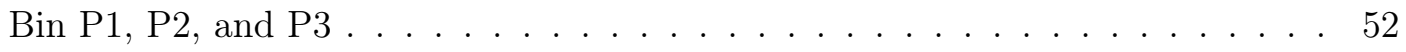

2-7 Observational and simulated regional climatologies; Bins P1 and A1 . . . . . 53

2-8 The frequency distribution of $\frac{\mu_{\Sigma} \text { at peak } r_{\Sigma}}{\text { peak } \mu_{\Sigma}} \ldots \ldots \ldots \ldots \ldots \ldots$

2-9 Simulated bio-physical correlations . . . . . . . . . . . . . . . 55

3-1 Pathways for eddy influence on biomass . . . . . . . . . . . . . . . 82

3-2 Simulated and observed eddy demographics . . . . . . . . . . . . . . . 83

3-3 Seasonal distribution of eddy tracks and biomass inventory . . . . . . . . . . . . 84

3-4 Variability in eddy anomalies . . . . . . . . . . . . . . . . . 85

3-5 Seasonal $M L D$ climatology and eddy anomalies. . . . . . . . . . . . . . 86

3-6 Seasonal $[\mathrm{Fe}]_{\Sigma}$ climatology and eddy anomalies. . . . . . . . . . . . . . 87

3-7 Seasonal $\mu_{\Sigma}$ climatology and eddy anomalies. . . . . . . . . . . . . . . 88

3-8 Vertical iron transport profiles . . . . . . . . . . . . . . . . . . . 89

$3-9$ Sources of anomalous iron . . . . . . . . . . . . . . . . . . 90

3-10 Seasonal variability of eddy anomalies in the deep mixing ACC . . . . . . . . 91

4-1 Pathways for eddy influence on biomass . . . . . . . . . . . . . . . . . 121

4-2 Hövmoller distributions of anomalous biological rate terms in Pacific eddies . . 122

4-3 Variability in rate anomalies as a function of season and background mixed layer depth . . . . . . . . . . . . . . . . . . . . . . 123

$4-4$ Distributions of anomalous rate terms . . . . . . . . . . . . . . 124

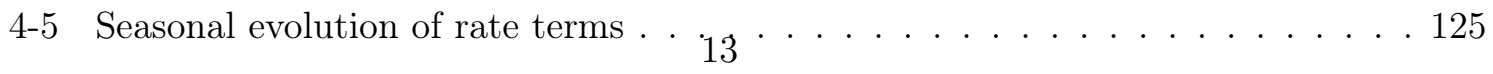


4-6 Anomalous biomass flux and concentration associated with vertical transport mechanisms . . . . . . . . . . . . . . . . . 126

4-7 Hövmoller distributions of anomalous physical transport mechanisms in Pacific eddies . . . . . . . . . . . . . . . . . . . . . . 127

4-8 Hövmoller distributions of anomalous biomass in Pacific eddies . . . . . . . . 128

4-9 Seasonal variability of depth resolved eddy anomalies in the deep mixing Pacific

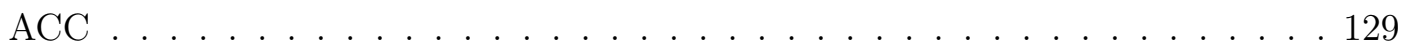

4-10 Hövmoller distributions of the correlation between anomalous surface biomass and the underlying mechanisms in Pacific eddies . . . . . . . . . . 130

$5-1 \quad$ Ocean iron fertilization schematic . . . . . . . . . . . . . . . 152

5-2 Flow Chart: The case against commercialization . . . . . . . . . . . 153

A-1 Cor Factor model fit . . . . . . . . . . . . . . . . . . 168

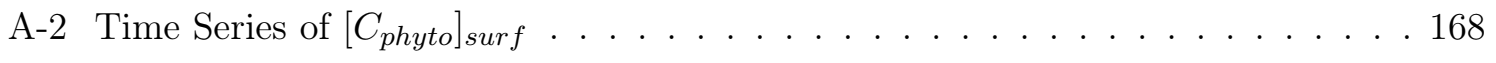

A-3 Model Skill . . . . . . . . . . . . . . . . . . . . . . . . . . 169

B-1 Seasonal $L_{\Sigma}^{I_{P A R}}$ climatology and eddy anomalies. . . . . . . . . . . . . . 172

B-2 Seasonal $L_{\Sigma}^{F e}$ climatology and eddy anomalies. . . . . . . . . . . . . . . 173

B-3 Seasonal $\frac{d[F e]}{d t}{ }_{M i x, \Sigma}$ climatology and eddy anomalies. . . . . . . . . . . . . . . 174

B-4 Seasonal $\frac{d[F e]}{d t}{ }_{W, \Sigma}$ climatology and eddy anomalies. . . . . . . . . . . . . . . . . . . . . . . . . . . . . . . . . . .

B-5 Seasonal $\Omega_{E k}$ climatology and eddy anomalies. . . . . . . . . . . . 176

C-1 Seasonal $\mu_{\Sigma}$ climatology and eddy anomalies. . . . . . . . . . . . . 178

C-2 Seasonal $l_{\Sigma}$ climatology and eddy anomalies. . . . . . . . . . . . . . 179

C-3 Seasonal $g r_{\Sigma}$ climatology and eddy anomalies. . . . . . . . . . . . . . 180

C-4 Seasonal mort $t_{\Sigma}$ climatology and eddy anomalies. . . . . . . . . . . . . . 181

C-5 Seasonal $a g g_{\Sigma}$ climatology and eddy anomalies. . . . . . . . . . . . . . 182

C-6 Seasonal $r_{\Sigma}$ climatology and eddy anomalies. . . . . . . . . . . . . . 183

C-7 Seasonal Mix $x_{C, S}$ climatology and eddy anomalies. . . . . . . . . . . . . . 184

C-8 Seasonal $W_{C, S}$ climatology and eddy anomalies. . . . . . . . . . . . 185

C-9 Seasonal $\left[C_{P h y t o}\right]_{S} S T I R$ climatology and eddy anomalies. . . . . . . . . . 186

C-10 Seasonal $\left[C_{P h y t o}\right]_{S} T R A P$ climatology and eddy anomalies. . . . . . . . . . 187

C-11 Seasonal $\left[C_{P h y t o}\right]_{S}$ climatology and eddy anomalies. $\ldots \ldots \ldots$. . . . . . 188

C-12 Seasonal $\Sigma C_{\text {Phyto }}$ climatology and eddy anomalies. . . . . . . . . . . . 189 


\section{LIST OF TABLES}

3.1 Frequency and magnitude of eddy anomalies . . . . . . . . . . . . . . . . 92

3.2 Spatially averaged anomalous iron supply rate from advection . . . . . . . . . 93

4.1 Seasonal distribution of frequency of eddies with positive anomalies . . . . . . . 131

4.2 Correlation between rate terms . . . . . . . . . . . . . . . . . 131

4.3 Spatially integrated anomalous biomass . . . . . . . . . . . . . . 132

A.1 Model Skill . . . . . . . . . . . . . . . . . . . . . . . . . 167 
Chapter 1

\section{INTRODUCTION}




\subsection{Motivation}

\subsubsection{Marine primary production, the carbon cycle, and phytoplankton ecosystem dynamics}

Phytoplankton, a broad classification for all autotrophic marine microorganisms, collectively form a vast aquatic forest that stretches across the ocean and helps sustain life on earth. Like terrestrial plants, phytoplankton fix inorganic carbon from carbon dioxide $\left(\mathrm{CO}_{2}\right)$ to form organic carbon compounds and release oxygen molecules $\left(\mathrm{O}_{2}\right)$ in the process as a byproduct. Although phytoplankton only constitute about $1 \%$ of global autotrophic biomass, they are responsible for almost $50 \%$ of the net primary productivity on the planet [Falkowski et al. 2000]. Marine primary production is in turn responsible for producing half the $\mathrm{O}_{2}$ we breathe [Falkowski et al. 2000] and populating the base of the oceanic food chain which supports the fisheries that millions of people rely on for food [Stock et al. 2017; Watson et al. 2013].

Further, the balance between carbon that is reduced by photosynthesis and oxidized by respiration known as the biological carbon cycle [Riebeek 2011] is responsible for regulating atmospheric $\mathrm{CO}_{2}$ levels and climate variability on glacial-interglacial timescales [Berner 1991; Sigman and Boyle 2000]. Variability in the biological carbon cycle is largely driven by marine bioegeochemistry and the biological pump [Berner 1991; Sigman and Boyle 2000], a pathway for medium-to-long term oceanic carbon sequestration [de la Rocha 2006]. Most of the carbon fixed by phytoplankton is rapidly recycled and released back to the atmosphere, but a small fraction $(\sim 15 \%)$ sinks and escapes the euphotic zone where it can remain sequestered in the deep ocean for hundreds of years before it is reminerialized [Laws et al. 2000].

Although phytoplankton clearly play an integral role in maintaining an oxygen rich atmosphere, a sustainable food supply, and a stable climate, there remains a great deal of mystery surrounding what controls variability phytoplankton ecosystem dynamics [Falkowski 1997]. Unlike terrestrial plants, phytoplankton rapidly turn over their population in a matter of weeks [Falkowski 2002], making them particularly sensitive to changes in their environment. Moreover, environmental variability is much more dramatic in the ocean than on the land. Physical and biogeochemical processes combine to constantly modify the growth conditions and grazing pressure that phytoplankton communities are subject to [Behrenfeld et al. 2013]. In turn, net phytoplankton population growth is often limited to short seasonal blooms [Longhurst 2006] but the mechanisms that shape the size and timing of these blooms highly variable and not fully understood [Rohr et al. 2017].

As climate change reshapes the physical [Jang et al. 2011] and biogeochemical [Feely et al. 2009] landscape of the oceans, the phenology of blooms and shape of marine ecosystems will also change [Sarmiento et al. 2004]. Better understanding the controls on phytoplankton 
ecosystem dynamics will help us understand whether or not marine primary production will feedback to buffer or amplify a changing climate, and ultimately how higher trophic levels and fisheries will respond [Chassot et al. 2010]. A variety of mechanisms compete to control phytoplankton ecosystem dynamics [Rohr et al. 2017], complicating our ability to draw direct lines of causality as to how and why blooms form and hampering our ability to predict how a changing environment will modify them. Here, with a focus on the Southern Ocean, I employ a suite of computational methods to study numerical simulations and remote sensing data to help disentangle the biophysical controls on phytoplankton ecosystem dynamics.

\subsubsection{The Southern Ocean}

Once the crown jewel of human exploration [Elzinga 1993], by the mid 1900s excitement over the success of the International Geophysical Year had begun to transform Antarctica, and the Southern Ocean writ large, into a cradle for international scientific collaboration [Howkins 2010]. Under protection by the newly established Antarctic Treaty System (ATS), Antarctica was formally codified as a continent for peace and science [Handbook of the Antarctic Treaty System n.d.]. Since the ATS was ratified in 1959, sweeping interdisciplinary research has revealed the Southern Ocean as a critical geophysical and biogeochemical node, together acting as a major control on earth's climate system [Turner and Bindschadler 2010].

Geophysically, the Southern Ocean is integral in mixing the global oceans and thereby transporting heat, salt, and nutrients across the world. Laterally, the Antarctic Circumpolar Current is the only substantial connection between earth's major ocean basins [Rintoul et al. 2001] and vertically, deep water formation in coastal polynas plays a critical role in driving global overturning circulation [Marinov et al. 2008]. Biogeochemically, Southern Ocean photo-

synthetic net community productivity is central to oceanic carbon storage and cycling [Treguer and Pondaven 2002]. Together, the Southern Ocean is capable of regulating earth's changing climate in a major way [Watson et al. 2014] and providing a direct feedback into the melting of the Western Antarctic Ice Sheet, which has already begun to lose mass and alone could contribute up 3 meters to global sea level rise [Feldmann and Levermann 2015].

Phytoplankton ecosystem dynamics are particularly interesting in the Southern Ocean. Unlike many ocean basins, phytoplankton division rates are limited by the micro-nutrient Iron, rather than the macro-nutrient Nitrogen [Boyd 2002; Martin et al. 1990]. Further, primary production is often limited by seasonal sea-ice coverage that dims the surface ocean for large portions of the year [Arrigo and van Dijken 2011], and strong grazing pressure by zooplankton [Dubischar and Bathmann 1997]. Together, Southern Ocean phytoplankton growth is often limited to short, seasonal blooms seasonal blooms [Longhurst 2006]. Nevertheless, Southern primary produvity is thought to play a critical role in regulating the global "biological pump" 
[Hauck et al. 2015], oceanic carbon storage [Marinov et al. 2008] and ultimately global climate dynamics [Chisholm 2000].

\subsection{Mechanistic drivers of net population growth and bloom phenology}

The size [Sullivan et al. 1993] and timing [Racault et al. 2012; Thomalla et al. 2011] of phytoplankton blooms is highly variable across the Southern Ocean. Understanding the role of Southern Ocean phytoplankton dynamics on larger scale biogeochemical and climate cycles is contingent on a better mechanistic understanding of why phytoplankton blooms emerge when and where they do. Phytoplankton bloom dynamics are controlled by a suite of interconnected physical, chemical, and biological mechanisms, which can drive phytoplankton population growth from the bottom-up and top-down. Bottom-up controls refer to anything that affects division rates such as nutrient abundance, light availability, or temperature limitation. In the Southern Ocean the dominant bottom-up controls are light [Nelson and Smith 1991] and iron limitation [Carranza and Gille 2015]. Top-down controls refer to anything that affects loss rates such as grazing, disease, viruses, mortality and aggregation (i.e. sinking). In the Southern Ocean grazing is particularly important [Deppeler and Davidson 2017; Rohr et al. 2017]. Net population growth is driven by subtle differences between two much larger competing rate terms [Behrenfeld et al. 2013]. In turn, the size and timing of biomass accumulation, is highly sensitive to a variety of mechanisms that can modify phytoplankton population dynamics both from the bottom-up and top-down. Untangling the influence of these controls, and uncovering where and when certain processes dominate, is instrumental in understanding how marine primary productivity will change in response to the shifting physical landscape of the Southern Ocean [Böning et al. 2008; Gille 2002].

\subsubsection{Mixed layer depth}

The predominant bio-physical control on phytoplankton growth is the seasonal mixing cycle, which can include mixed layer depths hundreds of meters deep in Southern Ocean during the winter [Dong et al. 2008]. The classical assumption has held that blooms are light limited, triggered by a shoaling of the mixed layer above some critical level of mean photosynthetically active radiation (PAR), thus eliminating light stress and allowing for high levels of community photosynthesis that exceed losses [Gran and Braarud 1935; Riley 1946; Sverdrup 1953]. This framework emphasizes 'bottom-up' controls, with growth conditions, namely light availability, primarily responsible for regulating variability in net population growth.

Observations of bloom initiation, occurring well before the mixed layer shoaled above this 
critical depth [Behrenfeld 2010; Boss and Behrenfeld 2010; Dale et al. 1999; Townsend et al. 1992] have, however, challenged the canonical association between the mixed layer depth, light limitation, and bloom initiation. For starters, deeper investigations into turbulent mixing rates [Huisman et al. 1999; Taylor and Ferrari 2011] reveal that the mixed layer depth is not always the best proxy for what is actively mixing, calling into question the actual residence time of phytoplankton cycling through the euphotic zone. Moreover, although deep mixing reduces light availability, it also can entrain high concentrations of iron from depth which may be needed to trigger Southern Ocean, iron-limited, blooms [Carranza and Gille 2015]. Finally, new evidence has suggested that deep mixing can trigger blooms from the top-down by diluting phytoplankton concentrations and reducing grazing efficiency [Behrenfeld 2010; Behrenfeld et al. 2013]. Behrenfeld et al. [2013] proposed that a bloom can be initiated during deep mixing even if growth conditions are deteriorating from exacerbated light stress, provided that grazing

rates are declining even faster as phytoplankton populations are diluted with plankton-free water entrained from depth.

\subsubsection{Eddies}

The Southern Ocean is characterized by a high degree of mesoscale activity [Meredith 2016; Stevens and Killworth 1992] and an abundance of coherent eddy features [Frenger et al. 2015]. Observational [Doney et al. 2003; Large 1998; McGillicuddy et al. 2007] and modeling [Anderson et al. 2011; Song et al. 2018] work agree that mesoscale processes help regulate biological productivity and dominate spatio-temporal variability in phytoplankton distributions [Doney et al. 2003; Glover et al. 2018]. Eddies can influence the biology within them through a variety of, often competing, processes that can both transport biomass and stimulate or suppress biological rates. [McGillicuddy 2016]. From a horizontal perspective, eddies can stir biomass as they rotate [Chelton et al. 2011; Doney et al. 2003; Glover et al. 2018] and trap biomass as they propagate [Early et al. 2011; Flierl 1981; Lehahn et al. 2011]. From a vertical perspective eddies can modify light and nutrient availability by modifying the mixed layer depth through isopycnal displacement [Hausmann et al. 2017; McGillicuddy 2016; Song et al. 2018] and induce upwelling or downwelling via eddy pumping [Falkowski et al. 1991; Franks et al. 1986] and eddy-induced Ekman pumping [Dewar and Flierl 1987; Gaube et al. 2014b]. Together, the confluence of various competing mechanisms leads to tremendous spatio-temporal variability in the size and direction of anomalous biomass within eddies [Frenger et al. 2018; Gaube et al. 2014b] and makes it difficult to constrain when and where different mechanisms dominate. 


\subsubsection{Sea Ice}

At high latitudes the introduction of seasonal sea ice can also regulate bloom dynamics. Sea ice imposes severe light limitation during the winter by attenuating incoming solar radiation before it reaches the surface ocean [Arrigo and van Dijken 2011], but also improves light availability during spring by stratifying the water column and reducing the mixed layer depth as it melts [Smith and Comiso 2008; Smith and Nelson 1985]. Further, the melt water flux can provide an impulse of nutrients, particularly iron, which can accumulate in the ice over the winter [Fennel et al. 2003; Lancelot et al. 2009; Sedwick and DiTullio 1997]. Together, it is not immediately clear if more ice and longer seasonal coverage should be expected to amplify or suppress a bloom, complicating our understanding of what will happen as sea-ice dynamics change [Stammerjohn et al. 2012]. The answer appears to vary with the strength of background wind conditions and the mean annual ice concentration [Montes-Hugo et al. 2009].

\subsubsection{Human intervention and Ocean Iron Fertilization}

In lieu of a dedicated mitigation effort to combat climate change [Clark 2012; Hovi et al. 2009; Shear 2018], some have suggested that we should use geoengineering to deliberately manipulate the climate system [Lynas 2011]. One proposed method for geoengineering the earth's climate is known as Ocean Iron Fertilization (OIF), which seeks to leverage inefficiencies in Southern Ocean phytoplankton productivity to increase oceanic uptake of atmospheric carbon dioxide $\left(\mathrm{CO}_{2}\right)$ and thereby sequester anthropogenic carbon for 10-100s of years [Gnanadesikan et al. 2003]. The idea is predicated on observations that in the iron-limited Southern Ocean, a relatively small amount of iron can stimulate a large increase in net primary production [Martin and Fitzwater 1988; Yoon et al. 2016]. The hope is that by strategically enriching the Southern Ocean with iron, a rampant increase in productivity could translate into a stronger biological pump, increased carbon exported to depth, and significant long term carbon storage. While, this notion is admittedly seductive, it is hardly advisable. Upper estimates of carbon storage suggest that perfectly efficient OIF could sequester as much as 50-100 ppm $\mathrm{CO}_{2}$ [Aumont and Ворр 2006], but it is unlikely much of the increased productivity stimulated by iron addition would be routed into long term export [Yoon et al. 2016]. Worse, any positive effects would likely be compensated by non-local effects once now macro-nutrient depleted water is upwelled at lower latitudes [Aumont and Bopp 2006; Gnanadesikan et al. 2003; Oschlies et al. 2010; Sarmiento and Orr 1991]. 


\subsection{Observational challenges and the role for models}

Relative to its size, the Southern Ocean shoulders a disproportionate role in regulating global biogeochemical [Marinov et al. 2008] and climate cycles [Chisholm 2000], yet remains largely understudied relative to lower latitude ocean basins. This stems directly from a host of challenges that befuddle traditional observational platforms [Abrahamsen 2014]. Harsh conditions characterized by high seas and strong storms coupled to remote locations far from support reduce the opportunity for shipboard observations throughout the year. Without major shipping lanes it is not possible to put passive monitoring equipment on commercial vessels and targeted oceanographic expeditions are much more logistically and financially demanding. In the winter, heavy sea ice coverage typically prevents shipboard observations altogether, leaving a massive seasonal hole in observations. The recent proliferation of ARGO floats [Gould et al. 2004] and drifters (e.g. Webb et al. [2001]) has begun to fill in the gaps but most floats and drifters are still subject to many of the same constraints, such as the inability to surface under ice. To date, the best bet to observe the Southern Ocean has been from space, nevertheless, satellite-based remote sensing observations are also constrained by their own set of challenges and limitations, particularly at high latitudes.

For starters, several factors work to blind satellite sensors from the oceans surface. Seasonal sea ice, cloud cover, and the positioning of the sun all work to prevent satellites from seeing the surface ocean, especially during the winter when ice and clouds are abundant and long nights combined with a sharp solar zenith angle at high latitudes keep satellites in the dark [Dierssen and Smith 2000]. Even when satellites can see the surface ocean unobstructed, there are limitations regarding what can be inferred about the biological system [Xavier et al. 2016]. Ocean color, observable passively from space, provides a reliable proxy for chlorophyll concentrations, but fails to consider variability in particle size distributions [Dierssen and Smith 2000], photo-acclimation [Sakshaug and Holm-Hansen 1986], or community composition, which all can change the chlorophyll to carbon ratio and bias estimates of biomass inferred from chlorophyll [Dierssen and Smith 2000]. More recently, direct estimates of carbon biomass have been made from particle back-scattering and absorption coefficients using the GSM spectral matching algorithm [Garver and Siegel 1997] and the carbon based productivity model [Behrenfeld et al. 2005; Westberry et al. 2008], however these are subject to their own set of biases and assumptions and have not yet been tested as thoroughly against in-situ observation as other chlorophyll-based products (e.g. Saba et al. [2011]).

Further, even if surface concentrations are assumed to be reliable, they are not always a good proxy for the total phytoplankton population, especially during deep mixing periods when dilution can reduce surface concentrations without changing the depth-integrated inventory [Behrenfeld et al. 2013; Rohr et al. 2017]. It is possible to infer the depth-integrated population 
by extrapolating surface concentrations over the mixed layer depth, but estimations of the mixed layer depth can not be observed remotely. In-situ observations have become more prevalent, but local data must be stitched together using state estimates (e.g. Mazloff et al. [2010]) or re-analysis products (e.g. Milutinović et al. [2009]), that are likely to miss much of the high frequency variability in seasonal and spatial mixing dynamics. Moreover, even once a mixed layer estimation is established, it is still often problematic to assume biomass is distributed uniformly across it. Results can be biased by biomass below a shallow mixed layer

[Behrenfeld et al. 2013], nonuniform biomass profiles and/or subsurface maxima [McGillicuddy et al. 2007; Siegel et al. 1999].

While observations are obviously foundational to our understanding of the Southern Ocean, the challenges detailed above limit their capacity to fully constrain Southern Ocean biogeochemical cycling and phytoplankton ecosystem dynamics, especially during the winter and at depth. In turn, many have pointed to the need to leverage numerical models to simulate what can not be observed (e.g. Frenger et al. [2018] and Gaube et al. [2014a]). Models, of course, can never perfectly describe nature and are limited by the processes and scales that are resolved. Nevertheless, numerical simulations are valuable in their ability to fill in gaps in the observational record, make predictions, and explicitly resolve the processes and mechanisms that can not be directly observed. In turn, models are clearly a necessary to compliment incomplete observations but must be tempered with an understanding of what is prescribed, parameterized and able to be resolved at the resolution of a particular integration. As the skill of coupled general circulations models continues to evolve in parallel with advances in the computing capacity needed to power them and the observational understanding needed to inform them, numerical simulations and broader computational methods will only become an increasingly important piece of Southern Ocean biogeochemical research.

\subsection{Thesis Overview}

Here, the state of the art Community Earth System Model (CESM) is used in conjunction with observations to help disentangle the controls on Southern Ocean phytoplankton ecosystem dynamics. First, in Chapter 2 the relative roles of bottom-up controls (division rates) and top-down controls (grazing rates) are examined in the context of net population growth rates and bloom phenology across the Southern Ocean. Results, simulated explicitly and supported by observations, demonstrate that blooms can be triggered by a variety of mechanisms, including mixed layer shoaling, mixed layer deepening, and sea ice retreat. Moreover, blooms do not always coincide with annually optimized division rates. In Chapter $\mathbf{3}$ and Chapter 4 eddies are identified and tracked in a global, multi-year, eddy-resolving integration of CESM. In Chapter 3 the mechanisms by which eddies can variably modify light and iron to stim24 
ulate or suppress the division rates of phytoplankton populations within them are examined. Results find that simulated eddies amplify divisions rates in Southern Ocean anticyclones, primarily by transporting iron from depth via eddy-induced Ekman Pumping. The opposite was found in cyclones. Next, in Chapter 4 the relative effects of eddy modified division rates, loss rates, net population growth rates, and physical transport mechanisms are all considered in the context of the biomass anomalies they combine to create. Different mechanisms are found to dominate at different times and different places, leading to tremendous spatio-temporal variability in the size and direction of eddy-induced biomass anomalies across the Southern Ocean. In Chapter 5 the merits of Ocean Iron Fertilization (OIF) are considered in light of the established complexity associated with the mechanistic underpinning of Southern Ocean phytoplankton blooms. An extensive review of the best-available science, international policy framework, and developing market forces confirms that lingering uncertainty coupled to concerns over efficacy, safety, and measurement prohibit a viable commercialization strategy. Finally, conclusions and future directions are presented in Chapter 6. 
CHAPter 2

\section{VARIABILITY IN THE MECHANISMS CONTROLLING SOUTHERN OCEAN PHYTOPLANKTON BLOOM PHENOLOGY IN AN OCEAN MODEL AND SATELLITE OBSERVATIONS}

This chapter was originally published as Rohr, T.R., Long, M.C., Kavanaugh, M.T., Lindsay, K., Doney, S.C. (2017). Variability in the mechanisms controlling Southern Ocean phytoplankton bloom phenology in an ocean model and satellite observations. Global Biogeochemical Cycles 31: 922-40.

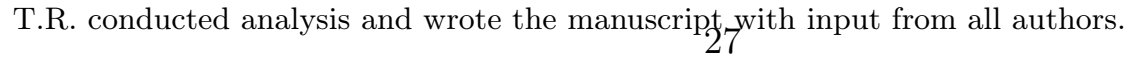




\begin{abstract}
A coupled global numerical simulation (conducted with the Community Earth System Model) is used in conjunction with satellite remote sensing observations to examine the role of topdown (grazing pressure) and bottom-up (light, nutrients) controls on marine phytoplankton bloom dynamics in the Southern Ocean. Phytoplankton seasonal phenology is evaluated in the context of the recently proposed 'disturbance-recovery' hypothesis relative to more traditional, exclusively 'bottom-up' frameworks. All blooms occur when phytoplankton division rates exceed loss rates to permit sustained net population growth, however the nature of this decoupling period varies regionally in CESM. Regional case studies illustrate how unique pathways allow blooms to emerge despite very poor division rates or very strong grazing rates. In the Subantarctic, southeast Pacific small spring blooms initiate early co-occurring with deep mixing and low division rates, consistent with the 'disturbance-recovery' hypothesis. Similar systematics are present in the Subantarctic, southwest Atlantic during the spring, but are eclipsed by a subsequent, larger summer bloom that is coincident with shallow mixing and the annual maximum in division rates, consistent with a 'bottom-up', light limited framework. In the model simulation, increased iron stress prevents a similar summer bloom in the southeast Pacific. In the simulated Antarctic zone $\left(70^{\circ} \mathrm{S}-65^{\circ} \mathrm{S}\right)$ seasonal sea-ice acts as a dominant phytoplankton-zooplankton decoupling agent, triggering a delayed but substantial bloom as ice recedes. Satellite ocean color remote sensing and ocean physical reanalysis products do not precisely match model predicted phenology, but observed patterns do indicate regional variability in mechanism across the Atlantic and Pacific.
\end{abstract}

\title{
Key Points
}

- CESM explicitly simulates variable pathways for Southern Ocean bloom formation driven by both bottom-up and top-down controls

- Simulated blooms can initiate amidst both strong or poor cell division rates depending on iron/light availability and trophic decoupling

- Implicit evidence in the remote sensing data supports these unique mechanistic pathways as well as a strong but variable grazing control

\section{$2.1 \quad$ Introduction}

Southern Ocean (SO) net community production (NCP) and the associated 'biological pump' play a critical role in oceanic carbon storage [e.g., Marinov et al. [2008]; Hauck et al. [2015]], 
carbon cycling [Siegel et al. 2014] and thus global climate dynamics [Chisholm 2000; Tréguer and Pondaven 2002]. In the SO, iron limitation [Boyd 2002; Martin et al. 1990], seasonal sea-ice cover [Arrigo and van Dijken 2011] and trophic dynamics constrain the net population growth of phytoplankton to seasonal blooms [Longhurst 2006]. Understanding these seasonal cycles in phytoplankton ecosystem dynamics is critical to our understanding of spatial and temporal variability in Southern Ocean NCP but is hampered by the complexity of interconnected physical, chemical, and biological controls.

Phytoplankton bloom dynamics are governed by bottom-up factors that control cell division rates, as well as top-down factors that control grazing and other loss rates. The net effect on seasonal net population growth is a balance between a number of different, and often opposing, bottom-up and top-down factors, and as a result, the timing of bloom initiation can vary substantially geographically and from year to year.

From a bottom-up perspective, light limitation can suppress division rates during deep winter mixing when depth averaged photosynthetically active radiation (PAR) over the mixed layer is low. For a bloom to occur, the springtime mixed layer depth must shoal above some critical level for depth-averaged PAR to drive levels of community photosynthesis above losses [Gran and Braarud 1935; Riley 1946; Sverdrup 1953]. In this context, phytoplankton biomass concentration is assumed to be well coupled to the depth-integrated inventory, and bloom initiation is typically estimated as the point in time when surface concentrations begin to increase substantially [Siegel et al. 2002]. However, some observations indicate that depth integrated biomass can increase before the mixed layer shoaled above this critical depth [Dale et al. 1999; Ellertsen 1993; Townsend et al. 1992] implying that bloom inception may be influenced by other mechanisms including dilution (or disturbance)-recovery [Fischer et al. 2014]. Mixed layer deepening can mediate 'top-down' controls on bloom development [Behrenfeld 2010; Behrenfeld et al. 2013] and decouple concentration-based and inventory-based growth rates. Bloom initiation, instead defined as the switch to positive net growth of the column-integrated population rather than surface concentration, can be triggered by subtle disequilibria in trophic coupling that decrease predation by zooplankton and promote sustained increases in phytoplankton biomass integrated over the mixed layer. This disequilibrium is modulated, in part, by entrainment during the fall and winter of phytoplankton depleted water during deep mixing, diluting the phytoplankton concentration on which grazing rates depend. Since grazing scales with prey concentrations, dilution can cause loss rates to drop below division rates; thus, deep mixing can actually maintain (or increase) the vertically integrated phytoplankton inventory [Behrenfeld 2010; Behrenfeld et al. 2013]. Under this 'disturbance-recovery' framework, bloom initiation can occur even while phytoplankton cell division rates are deteriorating (i.e., via increased light limitation from mixed layer deepening) so long as loss rates are declining at a faster rate (i.e., via dilution effects on predation). 
From a bottom-up perspective, deep mixing can also entrain nutrients from depth, replenishing surface levels of macro- and micronutrients [Carranza and Gille 2015], which are known to limit net primary productivity [Moore et al. 2013b]. In particular, throughout the Southern Ocean, iron is largely thought to be the dominant limiting nutrient, leading to expansive High Nitrate, Low Chlorophyll (HNLC) regions [Boyd and Ellwood 2010; de Baar et al. 1995, 2005; Martin et al. 1990]. However, the degree of iron limitation across the Southern Ocean is quite variable in space and time. Regional inputs from atmospheric dust deposition, anoxic coastal sediments, river runoff, and sea/glacial ice provide exogenous iron sources of varying significance [Boyd et al. 2012; Moore and Braucher 2008] that can modify bloom size and duration, and regulate the importance of deep mixing as an iron source.

Sea-ice can both hinder high latitude bloom development through severe surface light limitation [Arrigo and van Dijken 2011] and support cell division following ice melt via increased vertical stratification [Smith and Comiso 2008; Smith and Nelson 1985] and nutrient enrichment (particularly Fe) [Fennel et al. 2003; Lancelot et al. 2009; Sedwick and DiTullio 1997]. Note, however, nutrient enrichment from ice melt is not incorporated into many global-scale ocean biogeochemical models including the one used in this analysis (see 2.2.1). As Southern Ocean sea-ice dynamics are modified by climate change [Stammerjohn et al. 2012] these competing processes can lead to dramatically different effects on bloom dynamics. Depending on regional wind conditions and total ice concentration, decreased sea-ice could dampen or amplify the bloom [Montes-Hugo et al. 2009].

This complex suite of controls drives variability in the size [Moore and Abbott 2000; Sullivan et al. 1993] and timing [Racault et al. 2012; Thomalla et al. 2011] of phytoplankton blooms, which can affect trophic transfer and carbon export. Disentangling these controls is critical in understanding present, and predicting future, spatio-temporal variability in Southern Ocean NCP, and ultimately the role of marine autotrophs in a changing climate. In this study, model results and remote sensing observations are used to showcase how physical and biogeochemical controls can produce blooms via different mechanisms. In doing so, we test the suitability of the 'disturbance-recovery' hypothesis relative to more traditional, strictly 'bottom-up' systematics.

We first highlight the observed and simulated spatial variability in bloom size and timing across the Southern Ocean (see 2.3.1). Next we analyze how well coupled peak cell division rates are to peak net population growth rates and peak biomass inventory within the model to help infer the strength of bottom-up controls (see 2.3.1). Different mechanistic pathways are then explicitly examined by comparing the simulated climatologies of the relevant physics and biogeochemistry for four spatially averaged regional case studies simulated in CESM (see 2.3.2). Finally, the two model regions with the most dramatic differences and most complete remote sensing coverage are compared to the observations (see 2.3.3). 


\subsection{Methods}

\subsubsection{Numerical Experiments}

The Community Earth System Model (CESM) is a fully coupled, global climate model capable of simulating past, present, and future climate scenarios [Hurrell et al. 2013]. Here we utilize a preindustrial simulation with a recently improved treatment of photosynthesis under sea-ice [Long et al. 2015]. The new treatment better represents the effect of subgrid-scale heterogeneity in sea-ice thickness and water column irradiance on the non-linear (concave downward [Geider et al. 1998]) photosynthesis-irradiance curve, leading to a more realistic phenology and bloom magnitude. This 30-year simulation has been branched off a longer control simulation, and most model output was saved at high temporal resolution as daily means.

Except for the new sea-ice treatment, the component set up is identical to that of the 1850 control used in the CESM Large Ensemble project and described in detail by Kay et al. [2014]. The ocean component has nominal horizontal resolution of 1 degree and 10m vertical grid cells down to $250 \mathrm{~m}$. Sea-ice is treated using the CICE4 component [Hunke and Lipscomb 2008]. The ice model does not sequester iron or resolve any biogeochemistry. All atmospheric dust deposition over sea-ice is deposited directly into the surface water. The ocean biogeochemistry component (BEC) detailed by [Moore et al. 2013a] has been tested and validated against global datasets and shown to capture basin-scale spatial distributions in production, nutrient and chlorophyll concentrations [Doney et al. 2009; Moore et al. 2004, 2013a; Moore et al. 2013b], and key aspects of oceanic iron [Moore and Braucher 2008] and carbon cycling [Lima et al. 2014; Long et al. 2013; Moore et al. 2013a]. BEC features a single class of zooplankton and three phytoplankton functional types: diatoms, small phytoplankton, and diazotrophs. Diazotrophs, however, are strongly limited by temperature and therefore exist only in negligible concentrations in the Southern Ocean. Phytoplankton carbon biomass, $C_{\text {phyto }}($ mmol $C)$, is tracked in terms of grid cell concentration, $\left[\begin{array}{llll}C_{\text {phyto }}\end{array}\right]\left(\right.$ mmol $\left.C \mathrm{~m}^{-3}\right)$. Phytoplankton net population growth $\left(\frac{d\left[C_{\text {phyot }}\right]}{d t}\right)$ is governed by a photosynthetic net primary productivity term, $P_{\text {phyto }}$, and opposed by losses due to a grazing, $G_{\text {phyto }}$, linear mortality, mort $_{\text {phyto }}$, and quadratic mortality/aggregation, $a g g_{\text {phyto }}$, term, such that,

$$
\frac{d\left[C_{\text {phyto }}\right]}{d t}=P_{\text {phyto }}-G_{\text {phyto }}-\text { mort }_{\text {phyto }}-a g g_{\text {phyto }}
$$

where all terms are in $\left(\frac{m m o l C}{m^{3} d^{1}}\right)$ and phyto represents either of the two regionally dominant pools of autotrophic plankton resolved in the simulation, diatoms or small phytoplankton.

Grid cell net primary productivity $\left(P_{\text {phyto }}\right)$ is equal to a volumetric specific photosynthetic division rate, $\mu\left(d^{-1}\right)$, multiplied by the biomass concentration $\left(P_{\text {phyto }}=\mu\left[C_{\text {phyto }}\right]\right)$. The specific photosynthetic division rate is subject to temperature dependence $\left(L^{T}\right)$, multi-nutrient 
$(N, P, S i, F e)$ limitation $\left(L^{n}\right)$ and light availability $\left(L^{I_{P A R}}\right)$. Individual nutrient limitation terms vary from 0 to 1 as a nonlinear function of the available nutrient concentration and a class-specific nutrient specific half saturation coefficient. Multi-nutrient limitation is treated following Liebig's Law of the minimum [van der Ploeg et al. 1999] such that the maximum specific photosynthetic division rate $\left(\mu_{\text {phyto }}^{\max }\right)$ is only scaled by the most limiting nutrient limitation term rather than a multiplicative function of each. Note that because the nutrient limitation term scales the maximum growth rate lower values translate to greater nutrient stress. Nutrient stress is systematically less for small phytoplankton than for diatoms given the same nutrient concentration because of differences in the parameterization of their respective half saturation coefficients chosen to account for differences in their size and physiology [Sunda and Huntsman 1995]. Light limitation $\left(L^{I_{P A R}}\right)$ scales as a nonlinear function of photosynthetically available radiation, a dynamic $C h l: C$ ratio and the most constraining nutrient limitation term. Both the $C h l: C$ ratio and nutrient limitation terms are computed differentially for individual phytoplankton pools resulting in a species specific light limitation term. Together,

$$
\mu=\mu_{p h y t o}^{\max } L^{T} l^{n} L^{I_{P A R}}
$$

Grazing occurs on individual phytoplankton pools and is governed by a temperature dependent, non-linear function (Holling type III; [Holling 1959]) of the phytoplankton concentration such that,

$$
G_{\text {phyto }}=\mu_{z o o ; p h y t o}^{\max } L^{T}\left(\frac{\left[C_{\text {phyto }}\right]^{2}}{\left[C_{\text {phyto }}\right]^{2}+g_{\text {phyto }}^{2}}\right)\left[Z_{C}\right],
$$

where $\mu_{z o o ; p h y t o}^{\max }\left(d^{-1}\right)$ is the maximum zooplankton specific grazing rate on phytoplankton class phyto, $L^{T}$ is the dimensionless temperature dependency term, $\left[C_{\text {phyto }}\right]$ is the class-specific

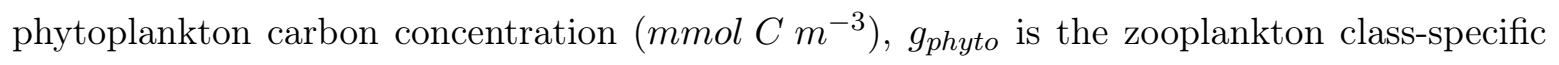

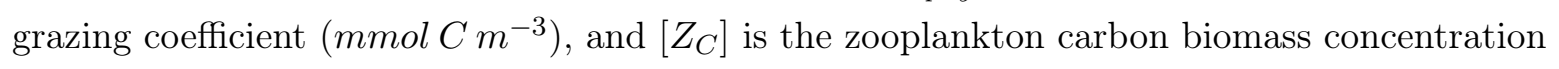
$\left(\mathrm{mmol} \mathrm{Cm}^{-3}\right)$. The class specification of the grazing parameterization ensures that zooplankton are more effective grazers on the small phytoplankton class, affording a relatively complex trophic control on phytoplankton at an affordable computational cost. Zooplankton is in turn governed by differential grazing on all three phytoplankton populations weighted by a nondimensional ingestion coefficient and a temperature dependent loss term. The nonlinearities of the grazing equation ensure that zooplankton become more efficient grazers as phytoplankton concentrations increase, eventually saturating towards a maximum grazing rate. 


\subsubsection{Remote sensing and reanalysis data}

We analyze ocean color remote sensing records compiled at 8-day resolution from 2005-2014 from the MODIS/Aqua satellite program. Observational indicators of phytoplankton abundance and growth include MODIS surface chlorophyll records, as well as phytoplankton carbon biomass and cell division rates estimated from particle back-scattering and absorption coefficients using the GSM spectral matching algorithm [Garver and Siegel 1997] and the carbon based productivity model (CbPM) outlined by Behrenfeld et al. [2005] and later improved and validated against global data sets by Westberry et al. [2008]. Observationally-based mixed layer depth estimates are sourced from HYCOM and FNMOC reanalysis projects [Milutinović et al. 2009]. HYCOM and FNMOC data sets are merged for improved spatial and temporal coverage. All aforementioned data has been sourced from the Oregon State Ocean Productivity web page [O'Malley 2015]. Daily sea-ice fractional coverage is estimated at $25 \mathrm{~km}$ resolution using the GSFC Bootstrap SMMR-SSM/I Version 2 time series [Comiso 2011; Comiso et al. 1997; Shang et al. 2010].

\subsubsection{Quantification of relevant rates and metrics}

\section{Phytoplankton metrics}

All modeled phytoplankton metrics (unless otherwise noted) are calculated for the sum of the two regionally dominant phytoplankton functional types, small phytoplankton and diatoms (See 2.2.1). CESM resolves both carbon and chlorophyll concentrations for each phyotoplakton pool using a dynamic Chl:C ratio to account for photoacclimation. In our analysis, phytoplankton abundance is quantified in carbon units (as opposed to chlorophyll) to avoid biases introduced by photoacclimiation. Note, however, that phytoplankton chlorophyll and carbon biomass have been shown to be well correlated in the Southern Ocean [Arrigo et al. 2008; Behrenfeld et al. 2005; Quéré et al. 2002]. To first order, qualitatively similar patterns in bloom size and timing appear in biomass estimated from the CbPM (see Fig. 2-1) and chlorophyll as observed by MODIS as well as the Coastal Zone Color Scanner and SeaWiFS [Moore and Abbott 2000; Sullivan et al. 1993]. During strong dilution events, however, it is possible that chlorophyll-based metrics will overestimate biomass as low light and photoacclimation increase the Chl:C ratio

Daily modeled and observed $\left[C h l_{\text {phyto }}\right]_{\text {surf }}$ values are used in our diagnostic analysis to approximate the euphotic depth following the empirical relationship developed by [Morel and Berthon 1989]. In the prognostic simulation, the full chlorophyll profile is used to calculate light penetration, however only surface chlorophyll concentrations $\left(\left[C h l_{\text {phyto }}\right]_{\text {surf }}\right)$ are saved at daily-mean resolution. 
Model simulated, depth resolved profiles of $\left[C_{p h y t o}\right]$ were only saved as monthly means, and therefore exact values of phytoplankton carbon inventory $\left(\Sigma C_{\text {phyto }}\right)$ integrated to timevarying mixed layer depth or euphotic depth can only be computed from monthly averages of biomass and depth. However, daily-mean simulated values are available for the phytoplankton carbon inventory integrated over the upper $100 \mathrm{~m}$ of the water column $\left(\Sigma C_{\text {phyto }}^{100}, \mathrm{mmolCm}^{-2}\right)$. Assumptions regarding the vertical distribution of biomass allow us to approximate both daily total water column inventory and surface concentrations. Following Behrenfeld et al. [2013], we assume that phytoplankton biomass is homogenously distributed across the greater of the mixed layer depth or euphotic depth $\left(Z_{\text {eu }}\right)$, referred to as the Profile Depth, $Z_{\text {profile }}=$ $\max \left(M L D, Z_{e u}\right)$. Biomass is assumed to drop to 0 below this depth. This assumption holds well for an actively mixing mixed layer that is deeper than the euphotic depth, though could be problematic for shallow mixed layers where phytoplankton biomass can persist in stratified water below the mixed layer depth [Behrenfeld et al. 2013; Boss and Behrenfeld 2010; Boss et al. 2008] or when there is little active mixing [Franks et al., 2015]. Specifically in the Southern Ocean, [Uitz et al. 2006] concluded from in-situ data sets that it was reasonable to assume a uniform distribution in deep, well-mixed water.

The water column phytoplankton carbon inventory is thus assumed equal to the vertical integral over $Z_{\text {profile }}\left(\Sigma C_{\text {phyto }}=\int_{\text {surface }}^{Z_{\text {profile }}}\left[C_{\text {phyto }}\right] d Z\right.$, mmol $\left.C^{-2}\right)$. For the model analysis, we use the daily-mean $100 \mathrm{~m}$ integral to approximate the inventory $\left(\Sigma C_{\text {phyto }}=\Sigma C_{\text {phyto }}^{100}\right)$ when $Z_{\text {profile }}<100 \mathrm{~m}$, recognizing that non-zero biomass in the depth range $Z_{\text {profile }}-100 \mathrm{~m}$ may violate our distribution assumption; however this assumption does not bias our approximation of total water column inventory. Additional biomass below $100 \mathrm{~m}$ is unaccounted for but unlikely to be significant given a shallow $Z_{\text {profile. }}$. When $Z_{\text {profile }}>100 \mathrm{~m}$ we extrapolate from $100 m$ to $Z_{\text {profile }}$ using the mean $0-100 m$ concentration $\left(\Sigma C_{\text {phyto }}=\left(\frac{Z_{\text {profile }}}{100} \Sigma C_{\text {phyto }}^{100}\right)\right)$. Here the assumption of a uniform profile is reasonable, particularly in CESM, given a deep, well-mixed layer that likely exceeds the euphotic depth.

Modeled surface carbon biomass concentrations $\left(\left[C_{\text {phyto }}\right]_{\text {surf }}, \mathrm{mmol} \mathrm{Cm}^{-3}\right)$ also were saved only as monthly means. Daily surface concentrations are first approximated by assuming a uniform depth profile across $Z_{\text {profile }}$ and dividing inventory by profile depth $\left(\left[C_{\text {phyto }}\right]_{\text {surf }}=\right.$ $\left.\Sigma C_{\text {phyto }} / Z_{\text {profile }}\right)$. This approximation can be problematic for shallow mixed layers where biomass may exist in the euphotic zone below the mixed layer depth. To improve our first approximation for shallow profiles $\left(Z_{\text {profile }}<100 m\right)$, estimated surface concentrations are further weighted by a spatially and profile depth dependent correction factor modeled from the simulated monthly surface concentration data (see Appendix A.1-A.3). Testing the skill of this approach with the monthly data demonstrated a strong match between the corrected approximate surface concentrations and true-modeled values across the Southern Ocean (see Appendix A.4). 
Remote sensing observations provide only surface concentrations, which are extrapolated to $Z_{\text {profile }}$ following [Behrenfeld et al. 2013], under the same uniform depth distribution assumption.

Volumetric phytoplankton specific net population growth rates, $r\left(d^{-1}\right)$, can be related to the specific photosynthetic division rate, $\mu\left(d^{-1}\right)$, by

$$
\frac{1}{\left[C_{\text {phyto }}\right]} \frac{d\left[C_{\text {phyto }}\right]}{d t}=r=\mu-l,
$$

where $l\left(d^{-1}\right)$ is the total specific loss rate composed of grazing $\left(l_{G r}\right.$, phyto $)$, phytoplankton mortality $\left(l_{\text {mort }}\right.$, phyto $)$, and aggregation $\left(l_{\text {agg, phyto }}\right)$ (see Eq. 2.1). Volumetric specific rate terms $(\mu, l)$ for a grid cell are calculated by normalizing the rate terms as they appear in Eq. 2.1 by the biomass concentration $\left(\mu=\frac{P_{\text {phyto }}}{\left[C_{\text {phyto }}\right.} ; l=\frac{l_{\text {Gr,phyto }}+l_{\text {mort }, \text { phyto }}+l_{\text {agg, }, \text { hhyto }}}{\left[C_{\text {phyto }}\right]}\right)$

Vertically-integrated population specific rates $\left(\mu_{\Sigma}, l_{\Sigma, \text { Gr,phyto }}, l_{\Sigma, \text { mort }, \text { phyto }}, l_{\Sigma, a g g, p h y t o} ; d^{-1}\right)$ are calculated by dividing the water column integrated daily rate terms $\left(\Sigma P_{\text {phyto }}, \Sigma G r_{\text {phyto }}\right.$, $\Sigma m o r t_{\text {phyto }}, \Sigma a g g_{\text {phyto }}$; mmol $\left.C m^{-2} d^{-1}\right)$ by the depth integrated phytoplankton carbon inventory $\left(\Sigma C_{\text {phyto }}\right)$. The total population specific loss rate $\left(l_{\Sigma}\right)$ is the sum of its components $\left(l_{\Sigma}=l_{\Sigma G r, p h y t o}+l_{\Sigma m o r t, p h y t o}+l_{\Sigma a g g, p h y t o}\right)$.

In the model, rate terms $\left(P_{\text {phyto }}, G r_{\text {phyto }}\right.$, mort $\left._{\text {phyto }}, a g g_{\text {phyto }}\right)$ are saved as daily mean $150 \mathrm{~m}$ depth resolved profiles. If $Z_{\text {profile }}>150 \mathrm{~m}$, we assume that $\operatorname{rates}(z>150 \mathrm{~m})=\operatorname{rates}(z=$ $150 \mathrm{~m})$. The population specific net growth rate for the depth-integrated inventory, $r_{\Sigma}\left(d^{-1}\right)$, can thus be explicitly calculated as

$$
r_{\Sigma}=u_{\Sigma}-l_{\Sigma}
$$

Without explicitly resolved rate terms, in the remote sensing data $r_{\Sigma}\left(d^{-1}\right)$, between two time points, $\mathrm{t}=0$ and $\mathrm{t}=1$, is calculated from temporal changes in phytoplankton biomass as follows,

$$
\begin{gathered}
r_{\Sigma}=\ln \left(\Sigma C_{\text {phyto-1 }} / \Sigma C_{\text {phyto-0 }}\right) / \Delta t, \text { if MLD is deepening and }>Z_{e u} \\
r_{\Sigma}=\ln \left(\left[C_{\text {phyto-1 }}\right]_{\text {surf }} /\left[C_{\text {phyto-0 }}\right]_{\text {surf }}\right) / \Delta t, \text { if MLD is shoaling or }<Z_{\text {eu }}
\end{gathered}
$$

where either concentration or vertical inventory is used, depending on mixed layer dynamics, to take into account dilution effects by entrainment of phytoplankton free water from below during deep mixed layer deepening. Bloom initiation is in turn defined as the onset of positive population net growth rates, $r_{\Sigma}>0$. A more comprehensive explanation can be found in [Behrenfeld 2010; Behrenfeld et al. 2013]. Note, the model $r_{\Sigma}$ as derived diagnostically from 
the simulation (Eq. 2.5) is able to explicitly isolate only biogeochemical factors (division and loss terms); however the formulation for the remote sensing data (Eq. 2.6) implicitly assigns all changes in population size to biological factors and thus neglects contributions from physical transport terms. Spatially averaged regional binning in 2.3.2 should, however, largely average out the effect of physical processes in the remote sensing data.

All nutrient and light limitation terms are explicitly resolved in the model and saved as daily mean depth profiles down to $150 \mathrm{~m}$. Mean community representative limitation terms are averaged over the $Z_{\text {profile }}$. Consistent with the rate term depth profiles, the deepest saved limitation term is extrapolated to depth if $Z_{\text {profile }}>150 \mathrm{~m}$.

\section{Sea-ice metrics}

The day of sea-ice retreat in a grid cell is defined as the day fractional ice coverage falls below 15\% [e.g. [Stammerjohn et al. 2008]] and remains below this threshold for at least 30 days; if this condition is not met, there is considered to be no retreat for that grid cell in that year. Alternatively, if annual ice does not exceed 15\% fractional coverage for at least 50 days there is considered no advance. Interannual correlations calculated with the day of retreat only consider years with a well-defined retreat date and grid cells with a minimum of ten such years.

\subsubsection{Model Skill Metrics}

CESM solutions are compared to observational values inferred from the remote sensing and reanalysis products. Various metrics are used to test model skill and are computed independently at each grid cell between $45^{\circ} \mathrm{S}$ and $55^{\circ} \mathrm{S}$. Ice coverage and higher solar zenith angles at higher latitudes lead to unsatisfactory seasonal remote sensing coverage relative to the lower latitudes. The model error is computed as the root mean square deviation of CESM and observational climatologies normalized by the observed maximum. The model peak magnitude/timing biases are computed as the difference between the maxima of CESM and observed climatologies. The peak magnitude bias is further normalized by the observed climatological maximum. Positive(negative) biases mean the model magnitude is over(under) estimated and timing is delayed(early). Correlation coefficients between CESM and observed climatologic time series are computed at each grid cell with zero lag. Metrics are reported as spatial averages and 'circumpolar' values refer to the mean of all grid cells between $45 \mathrm{~S}^{\circ}$ and $55 \mathrm{~S}^{\circ}$.

\subsubsection{Regional Bins}

We focus on the dynamics of four regionally averaged case studies chosen to provide explicit examples of some of the variability in bloom structure and mechanism simulated and observed 
in the Southern Ocean. All reported metrics are computed first at individual grid cells prior to spatial averaging. We examine a zonal comparison between the temperate SE Pacific (Bin $\mathrm{P} 1: 55^{\circ} \mathrm{S}-45^{\circ} \mathrm{S}, 100^{\circ} \mathrm{W}-80^{\circ} \mathrm{W}$ ) and SW Atlantic (Bin A1: $55^{\circ} \mathrm{S}-45^{\circ} \mathrm{S}, 60^{\circ} \mathrm{W}-40^{\circ} \mathrm{W}$ ) as well as a latitudinal progression from the temperate SE Pacific though the sub-polar SE Pacific into the seasonal sea-ice zone (Bins P1, P2: $65^{\circ} \mathrm{S}-55^{\circ} \mathrm{S}, 100^{\circ} \mathrm{W}-80^{\circ} \mathrm{W}$, and P3: $70^{\circ} \mathrm{S}-65^{\circ} \mathrm{S}, 100^{\circ} \mathrm{W}-$ $80^{\circ} \mathrm{W}$, respectively). All four regional bins are outlined in Fig. 2-1 \& 2-2. Our selection is justified in Sec. 2.3.2 in the context of results from 2.3.1.

\subsection{Results}

\subsubsection{Large-scale Southern Ocean patterns}

\section{Peak bloom size and timing}

Southern Ocean distributions of remotely sensed (Fig. 2-1a) and model simulated (Fig. 2-1c) maximum $\left[C_{\text {phyto }}\right]_{\text {surf }}$ display some important qualitative similarities. Both showcase dramatic spatial variability in bloom magnitude and a notable increase in peak $\left[C_{\text {phyto }}\right]_{\text {surf }}$ moving poleward, along regions of coastal upwelling, and in the southwest Atlantic basin (Fig. 2-1a, c). Quantitatively, the circumpolar model error is 66\%. The CESM biomass maxima are systematically too high (note scale of color bars), with a circumpolar peak magnitude bias of $143 \%$, and miss some smaller scale spatial structure likely related to mesoscale features of the Antarctic Circumpolar Current. In particular, the model error is worst in the SW Atlantic (180\% in Bin A1, see Fig. 2-1), however the amplification of the SW Atlantic basin relative to the SE Pacific is coherent between the simulation and observations. Accordingly, we focus here on qualitative differences in bloom development as opposed to absolute magnitudes, addressing these quantitative discrepancies further in 2.3.3.

Spatial variability is also apparent in peak bloom timing, with peak $\left[C_{\text {phyto }}\right]_{\text {surf }}$ generally occurring later at higher latitudes in both observations (Fig. 2-1b) and the simulation (Fig. 2-1d). However, the model predicts substantially earlier maxima in the open ocean than the observations. In particular, blooms in the SE Pacific (Bin P1 in Fig. 2-1) are biased by -53 days. The implications of this discrepancy in the context of bloom mechanics are also addressed in 2.3.3.

Depth integrated inventories provide a valuable, complementary picture of bloom dynamics. The circumpolar model error for $\Sigma C_{\text {phyto }}$ is $38 \%$. In both model and observations (see 2.3.3) simulated peak $\Sigma C_{\text {phyto }}$ does not necessarily align with the size or timing of peak $\left[C_{\text {phyto }}\right]_{\text {surf }}$. Notably, in the model many open-ocean regions exhibit peak $\Sigma C_{\text {phyto }}$ prior to peak $\left[C_{\text {phyto }}\right]_{\text {surf }}$ (e.g. $\sim 3$ weeks earlier in Bin P1 - Fig. 2-1d, f), while in the sea-ice zone (black contour - Fig. 2-1 - $2-37$ ) and parts of the SW Atlantic the peaks 
co-occur. While a larger magnitude of peak $\left[C_{\text {phyto }}\right]_{\text {surf }}$ typically corresponds with larger peaks in inventory, closer examination reveals this relationship is not linear. The ratio of $\operatorname{peak}\left(\Sigma C_{\text {phyto }}\right): \operatorname{peak}\left(\left[C_{\text {phyto }}\right]_{\text {surf }}\right)$ is highly variable, ranging from $10-30 \mathrm{~m}$ in the SW Atlantic (e.g. Bin A1) to upwards of $100 \mathrm{~m}$ in the SE Pacific (e.g. Bin P1) despite similar mean peak mixing depths between A1 (156m) and P1 (164m) indicating differences in timing and/or mechanism.

\section{Cell Division and Net Population Growth Rates}

Variability in the simulated co-evolution of cell division and net population growth rates reveals implicit differences in the underlying mechanisms controlling the variability in simulated bloom phenology displayed in Fig. 2-1. In a theoretically idealized system completely controlled from the bottom-up, population specific loss rates would be invariant in time and all variability in $r_{\Sigma}$ would be described by $\mu_{\Sigma}$. Fig. 2-2, however, shows a large degree of variability in the degree of coherence between $\mu_{\Sigma}$ and $r_{\Sigma}$ across the SO in CESM suggesting that a variety of different mechanisms control net population growth $\left(r_{\Sigma}\right)$.

In the SW Atlantic (in particular Bin A1) $r_{\Sigma}$ peaks just before peak $\mu_{\Sigma}$ (Fig. 2-2c), when $\mu_{\Sigma}$ exceeds $80 \%$ of the annual maximum (Fig. 2-2a). Following peak $\mu_{\Sigma}$ and $r_{\Sigma}$, biomass continues to accumulate for weeks (Fig. 2-2d) until $\mu_{\Sigma}$ drops below $\sim 50 \%$ of the annual maximum (Fig. 2-2b), finally receding below $l_{\Sigma}$ and terminating the bloom. This is consistent with a strong bottom-up control in which $r_{\Sigma}$ and $\mu_{\Sigma}$ tend to co-vary.

Similarly, in the seasonally ice-covered region (Fig. 2-2 - 30\% mean annual ice contour; Bin P3 - Fig. 2-2) peak $r_{\Sigma}$ and $\mu_{\Sigma}$ are well aligned (Fig. 2-2a, c). However, peak $\Sigma C_{\text {phyto }}$ occurs while $\mu_{\Sigma}$ is still quite high $(\sim>70 \%$ of annual maximum - Fig $\mathbf{2 - 2} \mathbf{b})$ indicating the stronger role of increasing loss rates and top-down controls in bloom shut down, opposed to $\mu_{\Sigma}$ simply receding below less variable $l_{\Sigma}$.

In contrast over much of the simulated open Southern Ocean, and in particular Bin P1, peak $r_{\Sigma}$ (Fig. 2-2c) and $\Sigma C_{\text {phyto }}$ (Fig. 2-2d) occur in quick succession when $\sim<50 \%$ of annual maximum and months before peak $\mu_{\Sigma}$. It follows that peak $r_{\Sigma}$ can be, but is not always, largely decoupled from the strict evolution of cell division rates - a direct reflection of bottom-up growth conditions. The decoupling of $r_{\Sigma}$ and $\mu_{\Sigma}$ emphasizes the importance of temporally variable loss rates and top-down controls.

\subsubsection{CESM Regional Case Studies: Zonal and Latitudinal Comparisons}

We highlight the explicit climatologies of several of the qualitatively different mechanisms implied in Fig. 2-2. Four regional case studies are chosen on the basis of 1 . Providing a zonal comparison and latitudinal gradient, 2. Exhibiting strong variability in bloom size and 
timing in the simulation and observations (see Fig. 2-1), 3. Exhibiting strong variability in the simulated coherence between $\mu_{\Sigma}$ and $r_{\Sigma}$ (see Fig. 2-2), 4. Highlighting two mechanistic pathways subject to debate in the current literature (The Critical Depth and DisturbanceRecovery Hypotheses) and 5. Falling in proximity to two Ocean Observatory Initiative (OOI) sampling sites to enable the possibility of future in-situ comparisons.

\section{Zonal Comparison: Temperate, Open-Ocean SE Pacific (Bin P1) vs. Temperate, Open-ocean SW Atlantic (Bin A1)}

The simulated temperate SE Pacific (P1) and SW Atlantic (A1) bins exhibit similar spring bloom dynamics. In both regions, a small spring bloom with localized peak $\Sigma C_{\text {phyto }}$ occurs in October after a positive division-loss decoupling $\left(r_{\Sigma}>0\right.$, Fig. 2-3d; $\mu_{\Sigma}>l_{\Sigma}$, Fig. 2-4a, b) in September. The early spring decoupling is coincident with the period of maximum deep mixing and strong light limitation (Fig. 2-4c, d). Despite favorable iron availability (relative to the annual cycle), phytoplankton division rates are flat or even deteriorating during bloom initiation and remain low and only slightly improving throughout the decoupling period (Fig. 2-4).

The behavior of the regions diverges over the late spring and summer, with much larger Atlantic $\left[C_{\text {phyto }}\right]_{\text {surf }}$ (Fig. 2-3c) and (Fig. 2-3e). These summertime biomass differences arise despite broadly similar patterns of seasonal mixing (Fig. 2-3a) and $\mu_{\Sigma}$ (Fig. 2-3f) and reflect the cumulative effect of a second positive division-loss decoupling during November and December in the Atlantic $\left(r_{\Sigma}>0\right.$, Fig. 2-3d; $\mu_{\Sigma}>l_{\Sigma}$, Fig. 2-4b) versus more closely coupled conditions in the Pacific $\left(r_{\Sigma} \sim 0\right.$, Fig. 2-3d; $\mu_{\Sigma} \sim l_{\Sigma}$, Fig. 2-4a). The regional variations may arise because the Atlantic bin experiences weaker spring-early summer iron limitation (Fig. 2-4c, d) and thus elevated $\mu_{\Sigma}$ during shoaling (Fig. 2-3c; Fig. 2-4a, b). Division rates eventually fall back below declining loss rates by early January in part because of self-shading and elevated light limitation (Fig. 2-4d) leading to bloom termination $\left(r_{\Sigma}<0\right.$, Fig. 2-3d; $\mu_{\Sigma}<l_{\Sigma}$, Fig. 2-4b)

\section{Latitudinal Progression: Sub-polar SE Pacific (Bin P2) \& Seasonal Ice Zone SE Pacific (Bin P3)}

Peak and annual mean $\mu_{\Sigma}$ progressively deteriorate poleward (from P1 to P3; Fig. 2-5f) due to colder temperatures and stronger annual mean light limitation (Fig. 2-4d, Fig. 2-6c, d) caused by the combined effects of weakening incoming solar irradiation, deeper mixing (P2) and/or seasonal sea-ice coverage (P3). Despite worsening cell division conditions, peak $\Sigma C_{\text {phyto }}$ increases at higher latitudes (from P1 to either P2 or P3) due to significantly longer and larger decoupling periods $\left(r_{\Sigma}>0 ; \mu_{\Sigma} l_{\Sigma}\right)$. In the sub-polar SE Pacific (P2) the sizable 
spring decoupling period is qualitatively similar to that of $\mathrm{P} 1$ but notably longer, allowing to continue to slightly outpace $l_{\Sigma}$ through December (Fig 2-6a). Although the sub-polar SE Pacific bloom peaks in early summer when division rates are high (like A1), it is initiated while cell division conditions are poor and eventually shutdown by increasing grazing rates opposed to deteriorating division rates (like P1).

In the higher latitudes (Bin P3, Fig. 2-5; Fig. 2-6) seasonal sea-ice coverage drives division (and in turn grazing) rates towards zero during the winter leading to standing phytoplankton stocks lower than in other bins. As ice recedes, improved surface PAR is accompanied by a rapidly shoaling mixed layer stabilized by fresh melt water input, together acting to improve light availability. Grazing rates (hampered by a small initial zooplankton population) lag significantly behind the surge in division rates (Fig. 2-6c) leading to a long decoupling period and the largest sustained population specific net growth rates of any analyzed bin. The bloom culminates in the largest peak $\Sigma C_{\text {phyto }}$ and $\left[C_{\text {phyto }}\right]_{\text {surf }}$ of all Pacific bins before grazing rates rebound and surpass division rates just prior to optimal division rates (Fig. 2-6b).

\subsubsection{Remote Sensing/Reanalysis Zonal Comparison}

The CESM simulation allows for the exploration of possible mechanisms decoupling division and loss rates to permit a bloom, but does not necessarily imply that these mechanisms are representative of dynamics in the real ocean in these specific regions. It is informative, therefore, to compare the regional variability between bin P1 and A1 in the model to that of the observations (Fig. 2-7) in a mechanistic context.

The model error in Bin P1, A1 and the complete circumpolar band is respectively 31\%, 21\% and $27 \%$ for $M L D ; 21 \%, 86 \%$ and $38 \%$ for $\Sigma C_{\text {phyto }} ; 20 \%, 180 \%$ and $66 \%$ for $\left[C_{\text {phyto }}\right]_{\text {surf }} ; 30 \%$, $44 \%$ and $35 \%$ for $r_{\Sigma} ; 25 \%, 35 \%$ and $35 \%$ for $\mu_{\Sigma}$. The correlation coefficient between CESM and observed climatologies averaged over $\mathrm{A} 1$ and $\mathrm{P} 1$ is .86 for $M L D, .52$ for $\left[C_{\text {phyto }}\right]_{\text {surf }}$, and .45 for $\Sigma C_{\text {phyto }}$. Model error is significant, but within the observed range, and despite high frequency variability of the relevant processes, CESM is able to capture some of the variability in important state variable at a fine daily resolution.

The largest model-data discrepancies in our regional analysis are in the bloom phenology of the temperate SE Pacific (Bin P1) and bloom magnitude of temperate SW Atlantic (Bin A1). In the temperate SE Pacific (P1), model $\left[C_{\text {phyto }}\right]_{\text {surf }}$ peak much earlier than in the observations (-64 day bias). Model $\Sigma C_{\text {phyto }}$ also peaks earlier than observations, but less so (-22 day bias). This can likely be attributed to discrepancies in simulated mixing dynamics, which resolve a shallower maxima (-43\% bias) and earlier minimum (-34 day bias); other physical factors, such as incident $P A R$ and $S S T$ seasonal climatologies, are generally consistent between model and

observations for P1 and A1 bins. Nevertheless, the mechanisms seem to align: peak inventory 
occurs while mixing is deeper and is followed by a delayed peak in concentration once mixing shoals.

In the temperate SW Atlantic (A1) the phenology of the summer $\Sigma C_{\text {phyto }}$ bloom is well aligned (-13 day bias); however the peak magnitude is much larger in the model (201\% bias). Interestingly though, this overshoot occurs despite dramatically higher observed division rates, implicitly necessitating much higher loss rates as well.

Discrepancies not-withstanding, some important similarities in the relative phenology and magnitude of P1 and A1 underscore the variability in mechanism seen in CESM. In both the observations and the simulation, the temperate SE Pacific (P1) has a larger ratio of peak $\Sigma C_{\text {phyto }}$ to peak $\left[C_{\text {phyto }}\right]_{\text {surf }}$ (Fig. 2-7e, c) than the temperate SW Atlantic (A1). Observations agree that the bloom is much closer to the mixing maxima and further from optimal division rates $\left(\mu_{\Sigma}\right.$ in P1 than in A1; Averaged over all observed individual years and relevant grid cells, peak $\Sigma C_{\text {phyto }}$ is 48 days closer to peak mixing and 32 days further from peak $\mu_{\Sigma}$ in $\mathrm{P} 1$ than $\mathrm{A} 1$.

Finally, observations show implicit evidence of a dynamic loss term supplementing bottomup processes as a critical control on biomass. Cell division rates are only $9 \%$ (in P1) and $12 \%$ (in A1) of their annual maximum during observed peak net population growth, implicitly requiring loss rates to be acting as an important control.

\subsection{Discussion}

The seasonal accumulation of depth-integrated biomass, referred to as a bloom, is ultimately dependent on a disequilibrium between cell division rates and loss rates. As many physical and biogeochemical factors interact to regulate a bloom, multiple mechanistic pathways can theoretically affect the magnitude, duration, and phasing of decoupling throughout the course of the year. Simulations, supported by observational indicators, illustrate variability in the type of mechanistic pathways possible throughout the SO.

The frequency distributions of $\frac{\mu_{\Sigma} \text { at peak } r_{\Sigma}}{\operatorname{peak} \mu_{\Sigma}}$ for all CESM SO grid cells (Fig. 2-8) reveals a wide range over which the delicate balance between $\mu_{\Sigma}$ and $l_{\Sigma}$ can yield peak net population growth rates - not just when $\mu_{\Sigma}$ is climaxing or $l_{\Sigma}$ is bottoming out as a singularly bottom-up or top-down paradigm would predict.

Case studies were selected to explicitly examine contrasting regional dynamics, but are not intended to precisely prescribe the entire set of SO dynamics. Our selection of openocean bins does however provide a diverse sample of the overall distribution (Fig. 2-8); The mean $\frac{\mu_{\Sigma} \text { at peak } r_{\Sigma}}{\text { peak } \mu_{\Sigma}}$ across our regional bins is 1.4 standard deviations below (P1), 1.6 standard deviations above (A1), and within .1 standard deviation (P2) of the distribution mean. P3 falls near the mean ( .3 standard deviations) of the distribution of ice covered grid cells. 
Practical considerations to omit in depth climatological analysis from the rest of the SO leave open the possibility of regions exhibiting dynamics distinct from those presented; however, this would only strengthen our argument that variable pathways can control bloom phenology.

In all open ocean bins (P1, P2, \& A1), CESM simulates an early spring bloom that initiates amidst poor cell division conditions and terminates while division rates are still improving. This early season bloom is concurrent with deep mixing and driven by a dilution-decoupling mechanism. In the North Atlantic, Behrenfeld et al. [2013] attributed strong positive interannual correlations between peak phytoplankton inventory and peak mixing depths in both observations and simulations as evidence of dilution-driven trophic decoupling. The same correlation computed from our model run across the Southern Ocean (Fig. 2-9a) implies that this mechanism is prevalent but does not dominate everywhere within the model domain. A strong positive correlation is found in $\mathrm{P} 1$ and $\mathrm{P} 2$ where the seasonal cycle is limited to a single, dilution driven bloom. In P2, where the correlation is slightly higher, a longer and larger deep mixing period (relative to P1) increases the magnitude of initial decoupling, thereby yielding a longer and larger early bloom, even as the mixed layer proceeds to shoal (Fig. 2-5; Fig. 2-6). The entrainment of nutrient rich deep water [Carranza and Gille 2015] and subsequent reduction of iron stress (Fig. 2-4c) helps bolsters very low, light-limited division rates (Fig. $\mathbf{2 - 4 a ) , ~ a n d ~ s u s t a i n s ~ t h i s ~ d e c o u p l i n g ~ p e r i o d ~ f r o m ~ t h e ~ b o t t o m - u p ; ~ h o w e v e r , ~ t h e ~ d i s p a r i t y ~ b e t w e e n ~}$ $\mu_{\Sigma}$ and $r_{\Sigma}$ necessitates the critical role of dilution effects. Most regions displaying a strong positive correlation between deep mixing and peak biomass (Fig. 2-9a) also overcome low cell division rates during peak net population growth (Fig. 2-2a) implying dilution driven processes are relevant elsewhere throughout the Southern Ocean.

In the A1 region of CESM, the correlation between peak inventory and mixing is notably weaker than in the Pacific sector; indeed, the simulation shows negative correlation in places (Fig. 2-8a) because a second, dominant summer bloom effectively overwhelms the contribution of the local dilution-driven spring bloom to the annual signal. This larger bloom initiates amidst favorable cell division conditions and does not shut down until cell division rates are in decline. It is concurrent with shallow mixing and resembles a more traditional, bottom-up, light limited bloom. Despite a disproportionate increase in summertime biomass the absolute magnitude of $\mu_{\Sigma}$ is only slightly elevated relative to P1 (a $3 \%$ increase in the annual peak and $7 \%$ increase in the annual mean - see Fig. 2-4a), necessitating the additional role for variable trophic interactions in distinguishing A1 and P1. The emergence of this summer bloom in the $\mathrm{A} 1$ region relative to $\mathrm{P} 1$ is attributed to a greater supply of iron which elevates division rates just enough to remain slightly decoupled from grazing during the light-favorable, shallow summer mixing period.

Both modeling [Misumi et al. 2014] and observational [Tagliabue et al. 2014] studies have found vertical mixing to be the dominant supply mechanism of new iron into the euphotic zone 
in the Southern Ocean. However, episodic iron inputs from atmospheric deposition [Gaiero et al. 2003] and heterogeneous sedimentary fluxes that supplement deep-water iron stocks [Moore and Braucher 2008; Tagliabue et al. 2009] contribute to regional variability in iron availability. Bin A1 experiences strong exogenous sources of iron relative to the Pacific from both seafloor sediments and aeolian dust deposition in nature [Gaiero et al. 2003; Moore and Braucher 2008; Tagliabue et al. 2009] and in the CESM. As a result, modeled iron limitation is consistently reduced in the Atlantic relative to the Pacific, with the most severe seasonal iron stress in A1 (Fig. 2-4d - black traces) being quantitatively on par with the most growth-conducive iron conditions in P1 (Fig. 2-4c). Reduced iron stress in Bin A1 keeps division rates slightly higher than in P1 during mixed layer shoaling, compensating for increasing self-shading and grazing as a large summer bloom accumulates in A1 but is absent in P1.

Transitioning in CESM from the perennially open ocean to the seasonally ice covered Antarctic zone, the distribution of $\frac{\mu_{\Sigma} \text { at peak } r_{\Sigma}}{\operatorname{peak} \mu_{\Sigma}}$ (see Fig. 2-8) has a much smaller range than in the open-ocean and is skewed toward higher values, indicative of a stronger bottom-up forcing driven by seasonal sea-ice. In the simulated SE Pacific (bin P3) bloom phenology is more in phase with ice than mixing dynamics. There is a clear mechanistic pathway for seasonal sea-ice to first suppress a bloom - via light limitation - before triggering a bloom during retreat - via relieved light limitation, melt water input, water column restratification, and potential trophic decoupling. Figure 2-9b shows the interannual correlation between the days of ice retreat and peak bloom inventory. The correlation changes from positive (i.e. a longer ice season correlating a with larger peak bloom) at low latitudes in the marginal ice zone to strongly negative further south, suggesting a shift in the balance between the dominance of the suppression and stimulation pathways. A similar latitudinal transition was observed by Montes-Hugo et al. [2009] along the Western Antarctic Peninsula, where declining sea-ice severity has led to opposite ecosystem responses in the north (smaller blooms) and the south (larger blooms).

Significant bias and error exist in both the remote sensing data and model output. Both the model and observations have a relatively coarse resolution that may average out more intricate ecosystem dynamics. Passive remote sensing data are forced to interpolate over cloudcovered days and reflect only daytime values. SO remote sensing is particularly challenging, with biases associated with ice, solar angle, seasonal coverage and changes in particle size distribution [Dierssen and Smith 2000]. Further, data are subject to the many assumptions implicit in the NPP and biomass algorithms [Behrenfeld et al. 2005, 2013; Westberry et al. 2008].

Model solutions are of course limited by computational constraints on the number of processes and constituents that can be resolved and the uncertainty associated with the complexity and parameterization of those that are. In particular, CESM lumps all zooplankton species 
into a single pool and underestimates SO winter mixing depths [Moore et al. 2013a]. Both could account for the discrepancies between observed and simulated bloom timing (P1) and magnitude (A1) seen in 2.3.3. Feedbacks within over-simplified food webs could alter the timing of trophic interactions, while the mixing bias could decrease the flux of deep nutrients, permit higher concentrations of overwintering zooplankton, and relax community light limitation [Doney et al. 2009]. Such simplifications can certainly influence phytoplankton division and population growth rates, limiting the ability of CESM to precisely predict robust regional estimates of $N P P$.

Nevertheless, simulated division and population growth rates fall within range of those observed in situ in the SO. Division rates derived from a variety of techniques (e.g. radiotracers, direct cell counts, etc.) ranged from roughly 1 to $1\left(d^{-1}\right)$ [Sakshaug and Holm-Hansen 1986; Smith et al. 1999; Spies 1987]. Net population growth rates measured by Boyd et al. [2001] were roughly an order of magnitude smaller and ranged from -.05 to $.1\left(d^{-1}\right)$.

Further, confidence in the qualitative nature of simulated bio-physical interactions (e.g. the grazing functional form [Doney et al. 1996; Fasham et al. 1990], species specific grazing rates, species specific light [Geider et al. 1998] and nutrient [van der Ploeg et al. 1999] limitation) insulate our more general qualitative conclusions about how bottom-up and top-down controls can respond to environmental forcing to yield a bloom in different ways. While our results may not offer a precise map of where mechanistic differences emerge in nature, they do provide a theoretical underpinning of how and why mechanisms may diverge across the Southern Ocean.

Despite significant quantitative disagreements, qualitative observational indicators in the remote sensing data support the model finding of regional variability in the mechanisms controlling bloom phenology. Observations and simulation agree that peak biomass in the temperate SE Pacific occurs much closer to the mixing maxima and cell division rate minimum than in the temperate SW Atlantic, where biomass inventory does not peak until cell division rates are much higher, and closer to their annual maximum, highlighting the significance of both top-down and bottom-up controls and strengthening the veracity of theoretical differences in how and why blooms can form. This message is not intended to diminish the importance of bottom up controls but rather to caution against neglecting the other term in the equation: $r_{\Sigma}=\mu_{\Sigma}-l_{\Sigma}$

Disentangling the role of these competing rate terms will prove only more important as climate change alters the landscape of the SO. In an intermodel comparison study [Laufkötter et al. 2015] found that 4 of 9 models predicted decreasing NPP under RCP 8.5 despite improving division rates resulting from declining biomass driven by stronger grazing rates. In turn, Laufkötter et al. [2015] stress the need to better understand the phenological and trophic coupling mismatches that can regulate phytoplankton biomass (and thus NPP) from the top down. Moving forward it is important to not only better understand these mechanisms but 
to understand which environmental landscapes support them, otherwise we risk making qualitatively backwards assumptions about the effect climate change will have on net community production.

\subsection{Conclusions}

Throughout the simulated Southern Ocean we observe several distinct mechanistic pathways underlying the spatial variability in bloom phenology, supported by indicators in the observational record. While bottom-up factors are clearly important, discrepancies in the seasonal evolution of division rates and net population growth rates in model and observations stress the equally important role of temporally varying loss rates. Both bottom-up and top-down controls play a significant, but variable role, with some blooms climaxing amidst annually optimal cell division rates and others amidst very poor cell division rates but even weaker grazing rates.

The 'disturbance-recovery' hypothesis [Behrenfeld et al., 2013] examined in the North Atlantic certainly appears relevant in the simulated Southern Ocean as well. In all CESM case study regions, population specific loss rates and division rates co-vary for much of the year; Periods of subtle disequilibria permit positive net population growth rates that are typically an order of magnitude lower than either the growth or loss terms. The dilution-recoupling mechanism looks to be prevalent during deep mixing in the simulated open ocean, however it is evident that other mechanistic decoupling pathways exist as well.

Despite some discrepancies between model and observations, there is ample and coherent evidence to support the notion that blooms can form via several distinct pathways across the Southern Ocean. Still, because results derive largely from a single model integration it is important to focus not on precisely where certain mechanisms emerge but rather on the theoretical implications of how these different pathways can inform the role of SO biogeochemistry in a changing climate. We hope this work will encourage other modeling groups to examine more carefully the biophysical mechanisms governing seasonal phenology and to routinely archive key model metrics at the required higher temporal resolution.

\section{Acknowledgements}

The CESM project is supported by the National Science Foundation and the Office of Science (BER) of the U.S. Department of Energy. Computing resources were provided by the Climate Simulation Laboratory at NCAR's Computational and Information Systems Laboratory (CISL), sponsored by the National Science Foundation and other agencies. This research was enabled by CISL compute and storage resources. TR was supported by an NDSEG graduate 
fellowship. SCD and MTK acknowledge support from the National Aeronautics and Space Administration Ocean Biology and Biogeochemistry Program (NNX14AL86G) and the National Science Foundation Polar Programs award 1440435 (Antarctic Integrated System Science) to the Palmer LTER program. Please contact trohr@mit.edu for further questions or to access to data. 


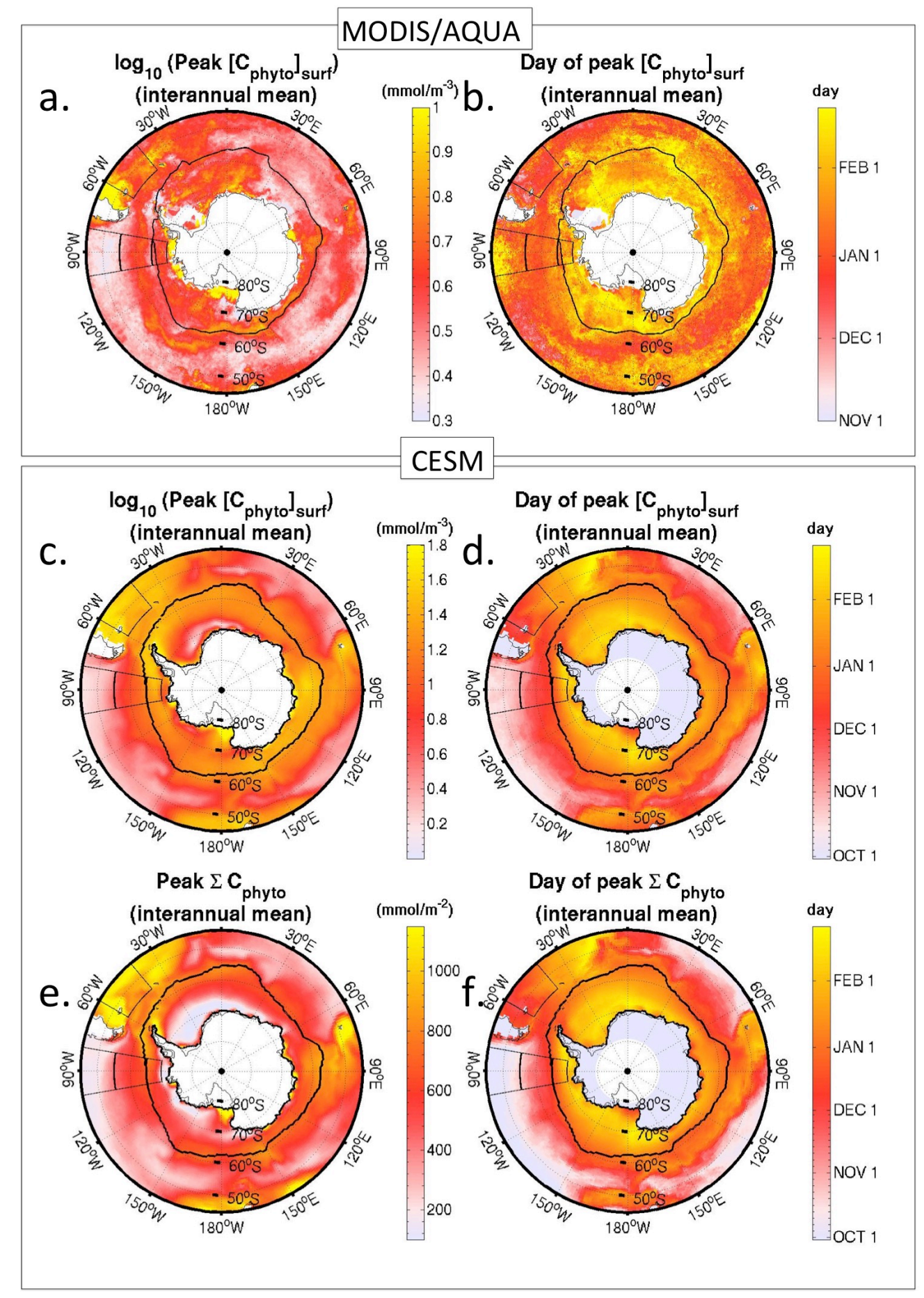

Figure 2-1: Peak bloom size and timing. Climatological mean phytoplankton (a) peak surface carbon biomass concentration and (b) day of peak surface carbon biomass concentration plotted from the MODIS/AQUA satellite record. (c) and (d) plot the same metrics from the CESM simulation. Note that subplots $(\mathbf{a})$ and (c) are plotted in $\log _{10}$ scale to better capture the spatial distribution. (e) and (f) plot the magnitude and timing of peak carbon biomass inventory as simulated by CESM. In all subplots, the region that sees, on average, at least $15 \%$ mean annual fractional ice coverage is outlined, along with regional bins $\mathrm{P} 1\left(55^{\circ} \mathrm{S}-45^{\circ} \mathrm{S}, 100^{\circ} \mathrm{W}-80^{\circ} \mathrm{W}\right), \mathrm{P} 2\left(65^{\circ} \mathrm{S}-55^{\circ} \mathrm{S}, 100^{\circ} \mathrm{W}-80^{\circ} \mathrm{W}\right)$, and P3 $\left(70^{\circ} \mathrm{S}-65^{\circ} \mathrm{S}, 100^{\circ} \mathrm{W}-80^{\circ} \mathrm{W}\right)$ in the Pacific and bin $\mathrm{A} 1\left(55^{\circ} \mathrm{S}-45^{\circ} \mathrm{S}, 60^{\circ} \mathrm{W}-40^{\circ} \mathrm{W}\right)$ in the Atlantic. 

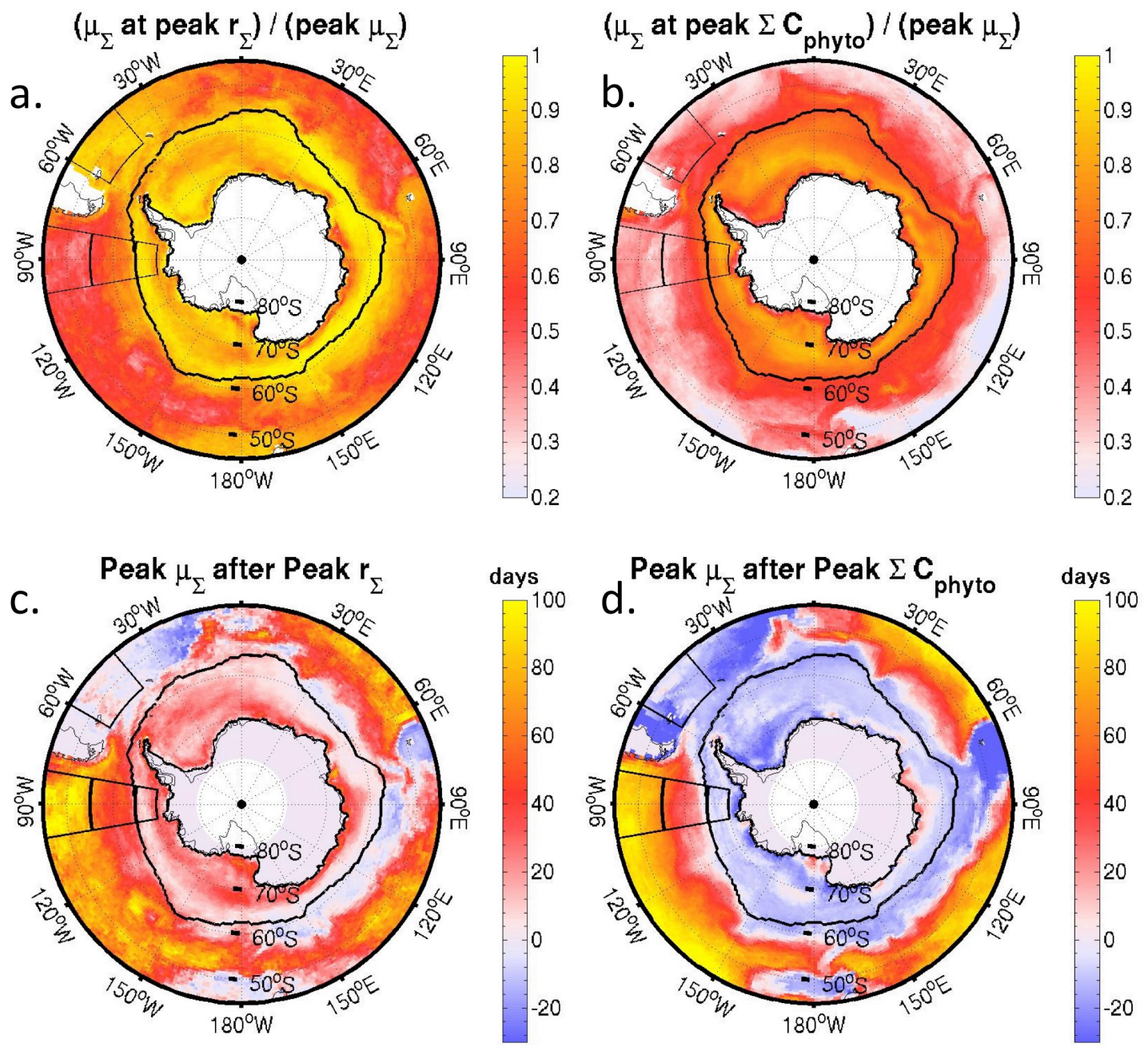

Figure 2-2: Relative size and timing of depth-integrated phytoplankton specific growth rates as simulated in CESM. Ratio of population specific division rate $\left(\mu_{\Sigma}\right)$ to seasonal peak $\mu_{\Sigma}$ at time of (a) peak net population specific growth rate $r_{\Sigma}$ and (b) peak biomass inventory $\left(\Sigma C_{\text {phyto }}\right)$. Number of days $\mu_{\Sigma}$ occurs after (c) peak $r_{\Sigma}$ and (d) peak $\Sigma C_{\text {phyto. }}$. Time series of $\mu_{\Sigma}$ and $r_{\Sigma}$ are first smoothed with a 7-day Hanning window, and metrics are averaged over 30 years of model output. 

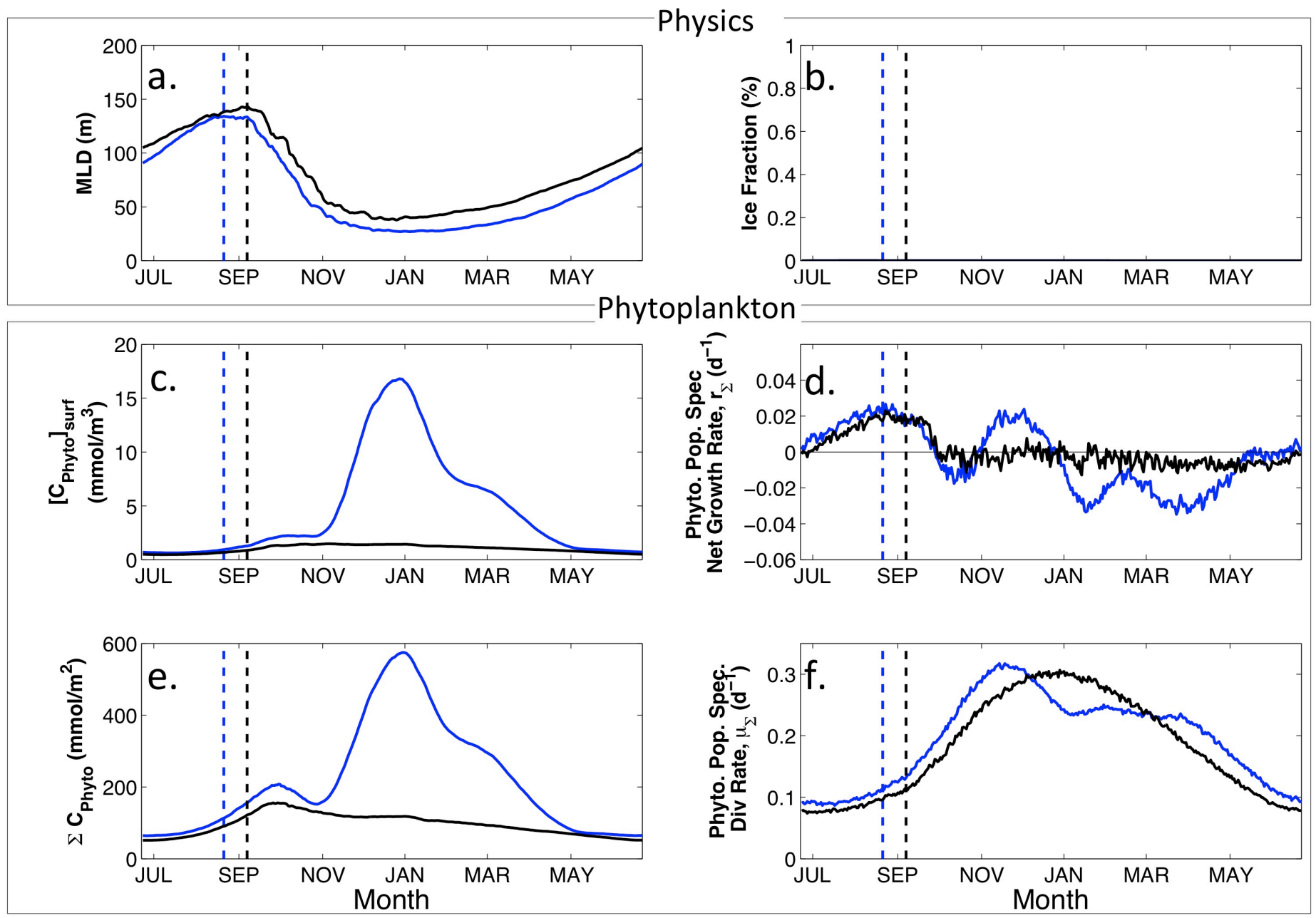

Region P1 (45-55S x 100-80W)—Region A1 (45-55S x 40-60W)

Figure 2-3: Regionally averaged, seasonal climatologies simulated by CESM for A1 and P1. (a) $M L D$ (b) ice fraction (not applicable) (c) $\left[C_{\text {phyto }}\right]_{\text {surf }}$ (d) $r_{\Sigma}(\mathbf{e}) \Sigma C_{\text {phyto }}$ (f) $\mu_{\Sigma}$. The SE Pacific bin $\left(\mathrm{P} 1: 55^{\circ} \mathrm{S}-45^{\circ} \mathrm{S}, 100^{\circ} \mathrm{W}-80^{\circ} \mathrm{W}\right)$ is plotted in black, and the SW Atlantic Bin (A1: $55^{\circ} \mathrm{S}-45^{\circ} \mathrm{S}$, $60^{\circ} \mathrm{W}-40^{\circ} \mathrm{W}$ ) in blue. The timing of the climatological maximum mixed layer depth is marked by a dashed line of the according color. 
Region P1

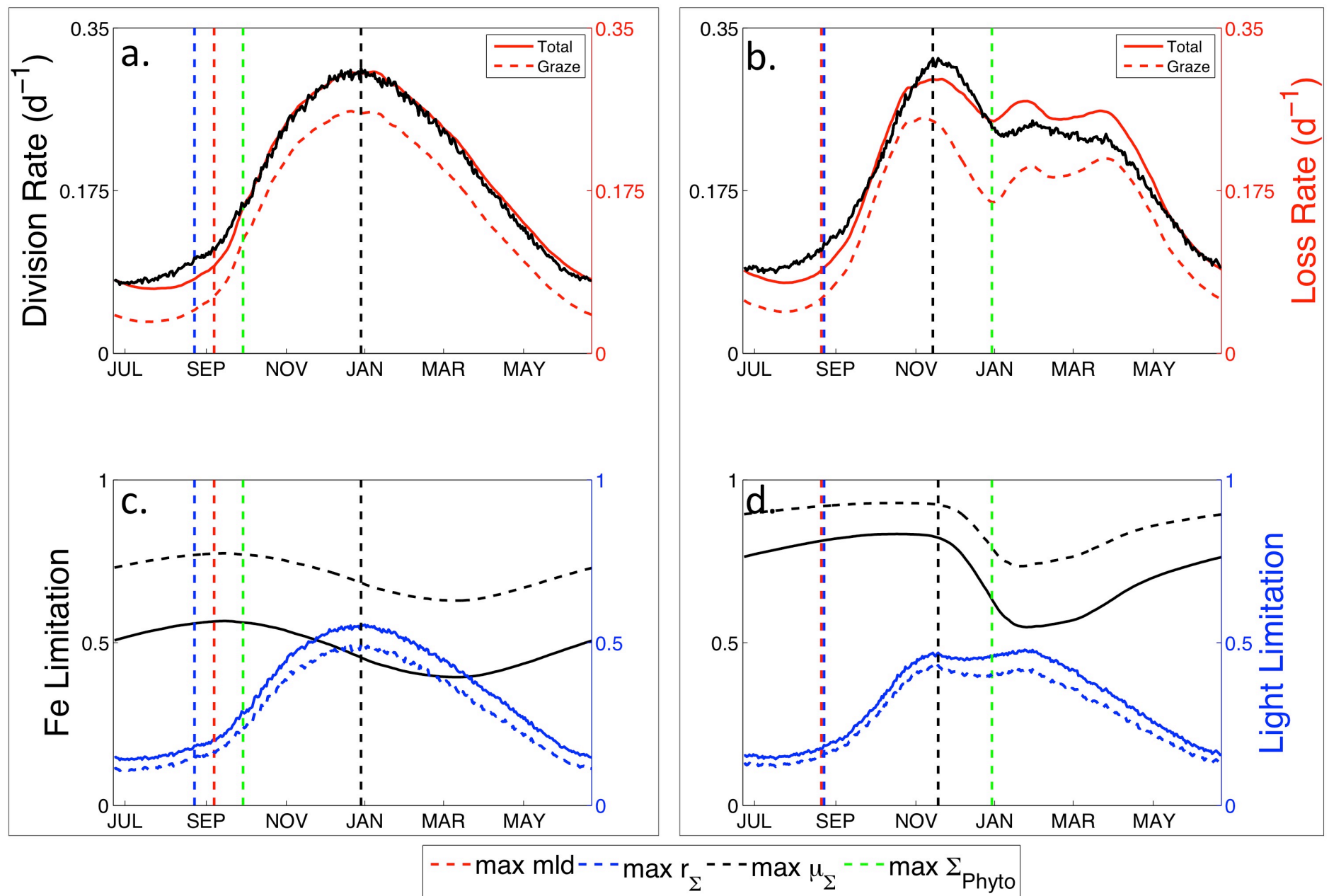

Figure 2-4: Seasonal climatologies of phytoplankton rate $(\mathbf{a}, \mathbf{b})$ and limitation $(\mathbf{c}, \mathbf{d})$ terms simulated by CESM averaged over Bin P1 $(\mathbf{a}, \mathbf{c})$ and A1 $(\mathbf{b}, \mathbf{d})$. (a, b) Population specific cell division rate (black), total loss rate (red solid), and grazing rate (red dashed). (c, d) Limitation terms averaged over the profile depth $\left(Z_{\text {profile }}\right)$ for iron (black; consistently the most limiting nutrient) and light (blue) with diatom (solid) and small phytoplankton (dashed). Vertical dashed lines refer to the climatologic timing of the maximum $M L D$ (red), population specific net growth rates (blue), population specific division rates (black) and phytoplankton biomass inventory (green). 


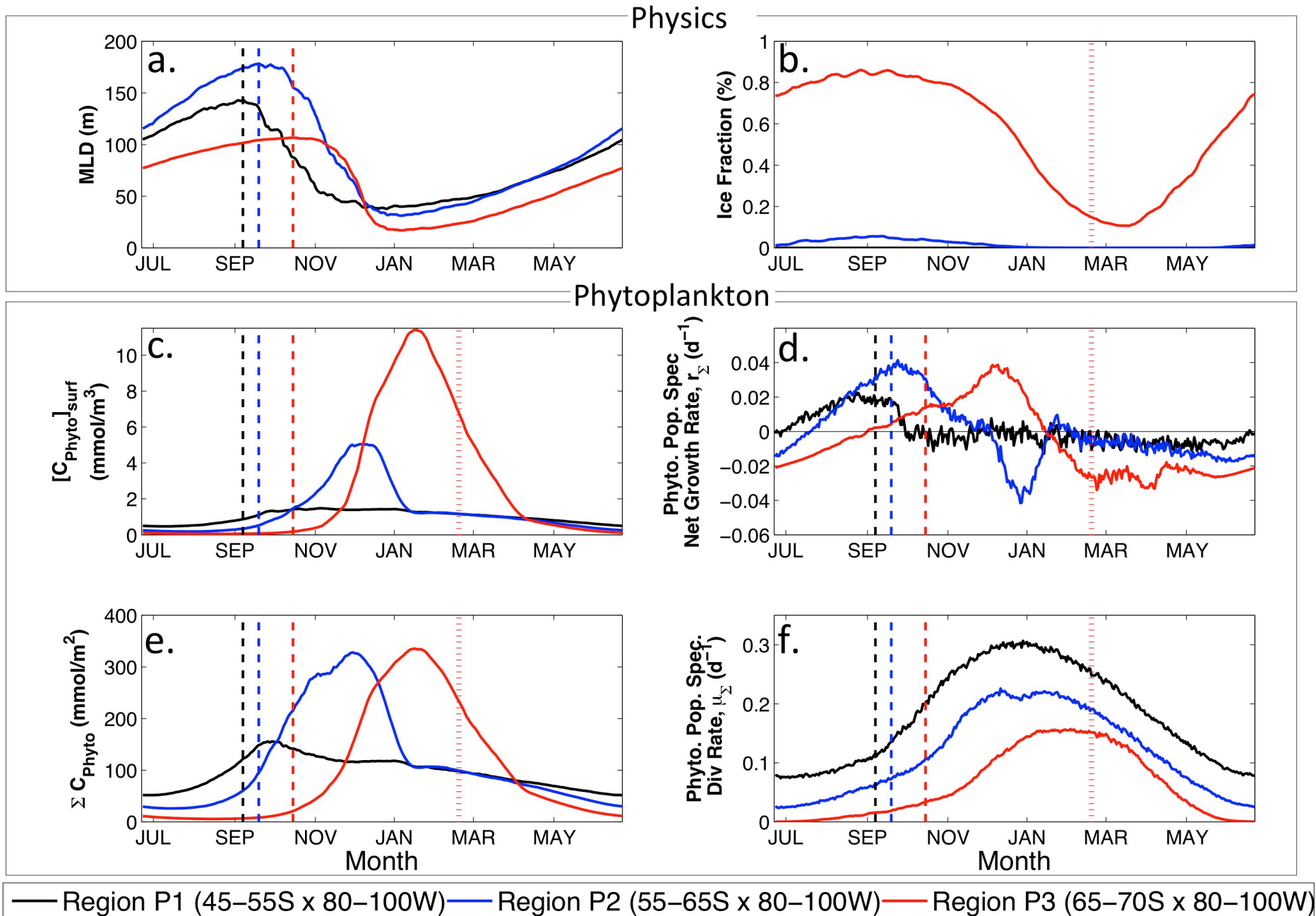

Figure 2-5: Regionally averaged, seasonal climatologies simulated by CESM for P1, P2, and P3. (a) $M L D$ (b) ice fraction (not applicable) $(\mathbf{c})\left[C_{\text {phyto }}\right]_{\text {surf }}(\mathbf{d}) r_{\Sigma}(\mathbf{e}) \Sigma C_{\text {phyto }}(\mathbf{f}) \mu_{\Sigma}$. The lowest latitude bin $\left(\mathrm{P} 1: 55^{\circ} \mathrm{S}-45^{\circ} \mathrm{S}, 100^{\circ} \mathrm{W}-80^{\circ} \mathrm{W}\right)$ is plotted in black, the 'middle' latitude bin $\left(\mathrm{P} 2: 65^{\circ} \mathrm{S}-55^{\circ} \mathrm{S}\right.$, $\left.100^{\circ} \mathrm{W}-80^{\circ} \mathrm{W}\right)$ in blue and the highest latitude bin $\left(70^{\circ} \mathrm{S}-65^{\circ} \mathrm{S}, 100^{\circ} \mathrm{W}-80^{\circ} \mathrm{W}\right)$ in black. The timing of the climatological maximum mixed layer depth is marked by a dashed line of the according color, and a color coated dotted line marks the climatologic timing of sea-ice retreat where applicable (here only in Region P3). 
Region P2

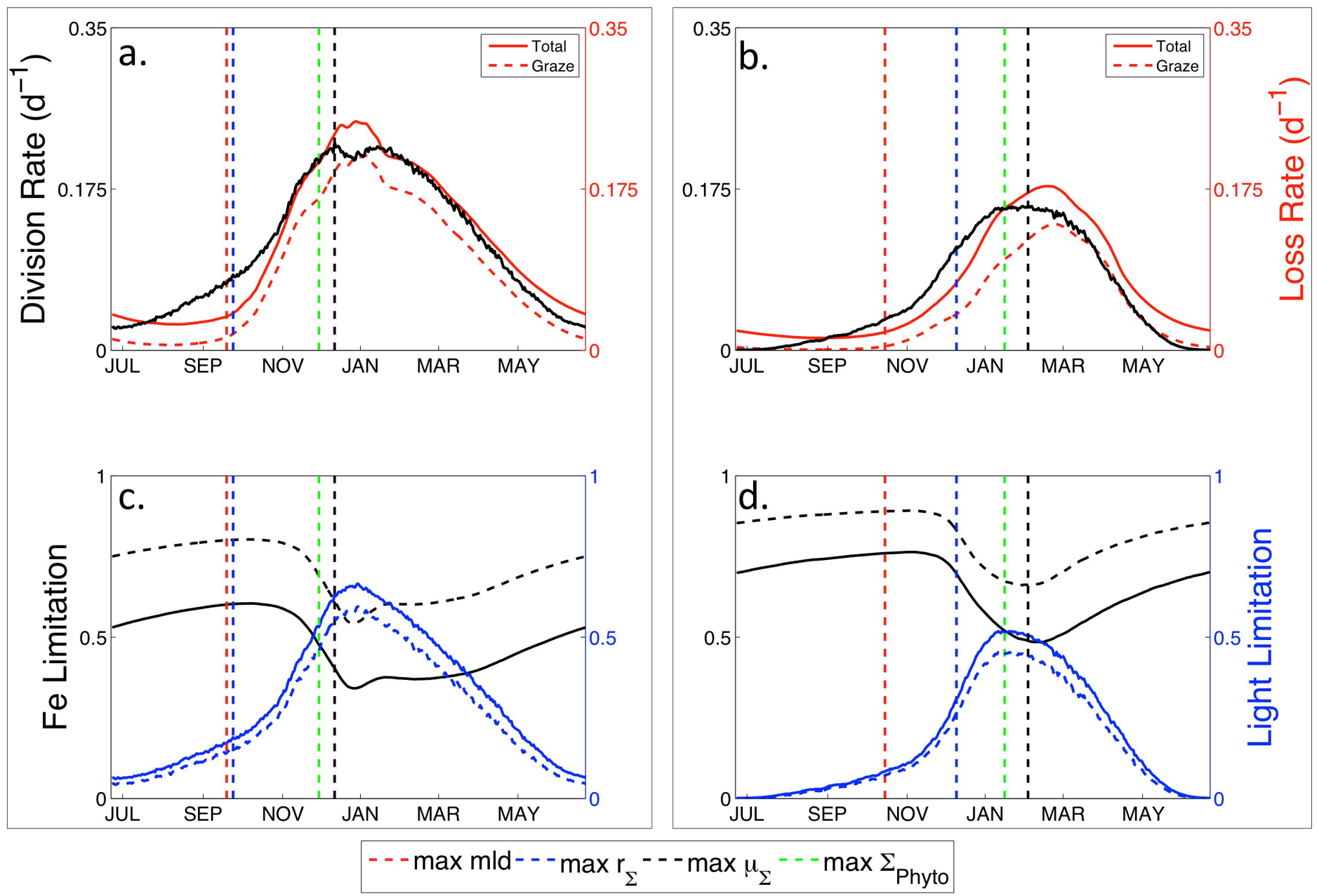

Figure 2-6: Seasonal climatologies of phytoplankton rate $(\mathbf{a}, \mathbf{b})$ and limitation $(\mathbf{c}, \mathbf{d})$ terms simulated by CESM averaged over Bin P1 $(\mathbf{a}, \mathbf{c})$ and A1 $(\mathbf{b}, \mathbf{d})$. (a, b) Population specific cell division rate (black), total loss rate (red solid), and grazing rate (red dashed). (c, d) Limitation terms averaged over the profile depth $\left(Z_{\text {profile }}\right)$ for iron (black; consistently the most limiting nutrient) and light (blue) with diatom (solid) and small phytoplankton (dashed). Vertical dashed lines refer to the climatologic timing of the maximum $M L D$ (red), population specific net growth rates (blue), population specific division rates (black) and phytoplankton biomass inventory (green). 


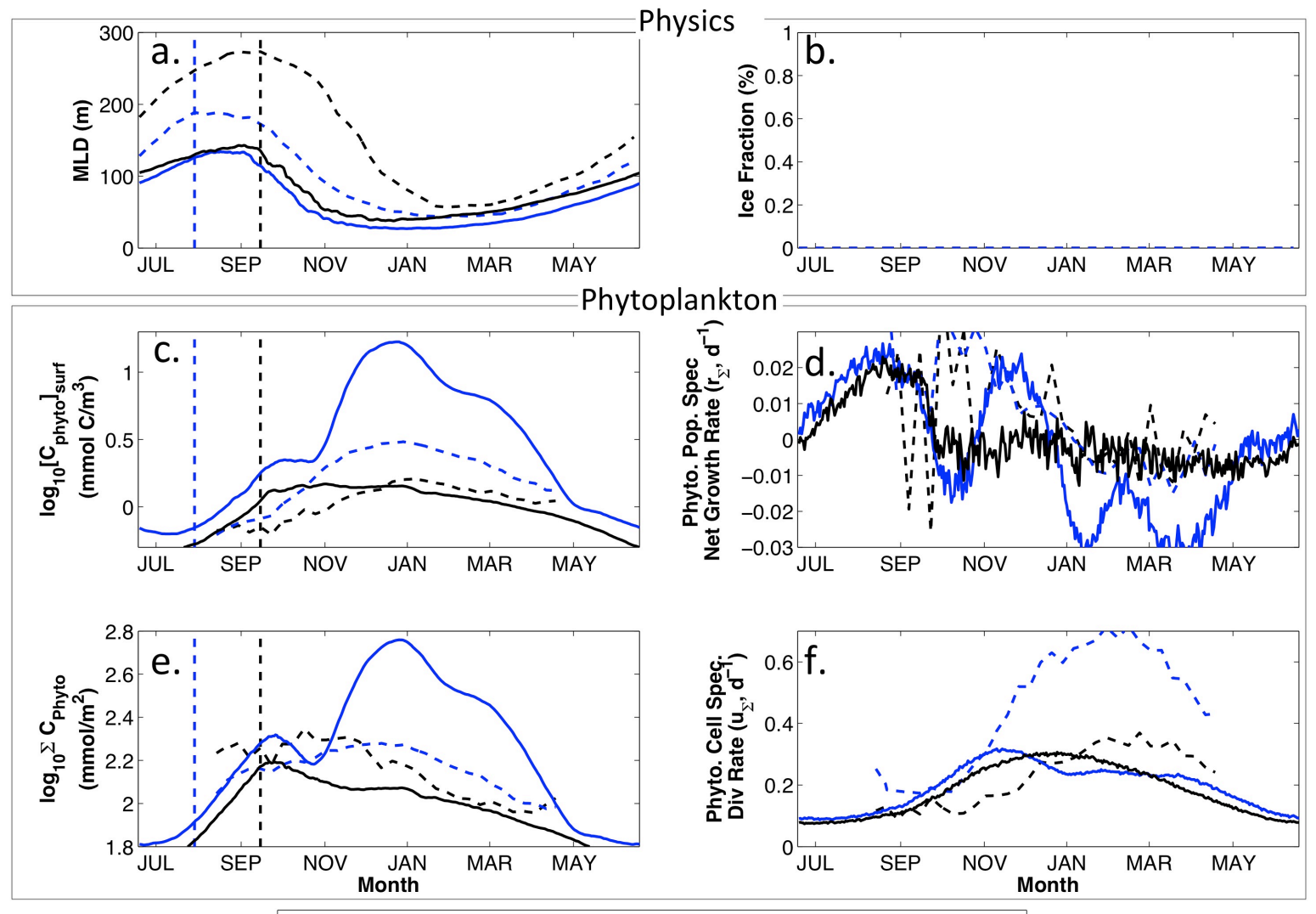

-P1 - CESM-A1 - CESM--- P1-Obs--- A1-Obs

Figure 2-7: Observational and simulated regional climatologies for P1 (Black) and A1(Blue) (a) HYCOM/FNMOC reanalyzed (dashed) and simulated (solid) MLD, MODIS observed (dashed) and simulated (solid) phytoplankton (c) biomass concentrations, (d) population specific net growth rates, (e) inventories, (f) and population specific division rates. A dashed line of the according color marks the timing of the climatologic HYCOM/FNMOC reanalyzed maximum mixed layer depth in a, $\mathbf{c}$ and e. Simulated traces are identical to Fig. 3, however concentration and inventory are now plotted on $\log$ scale. 


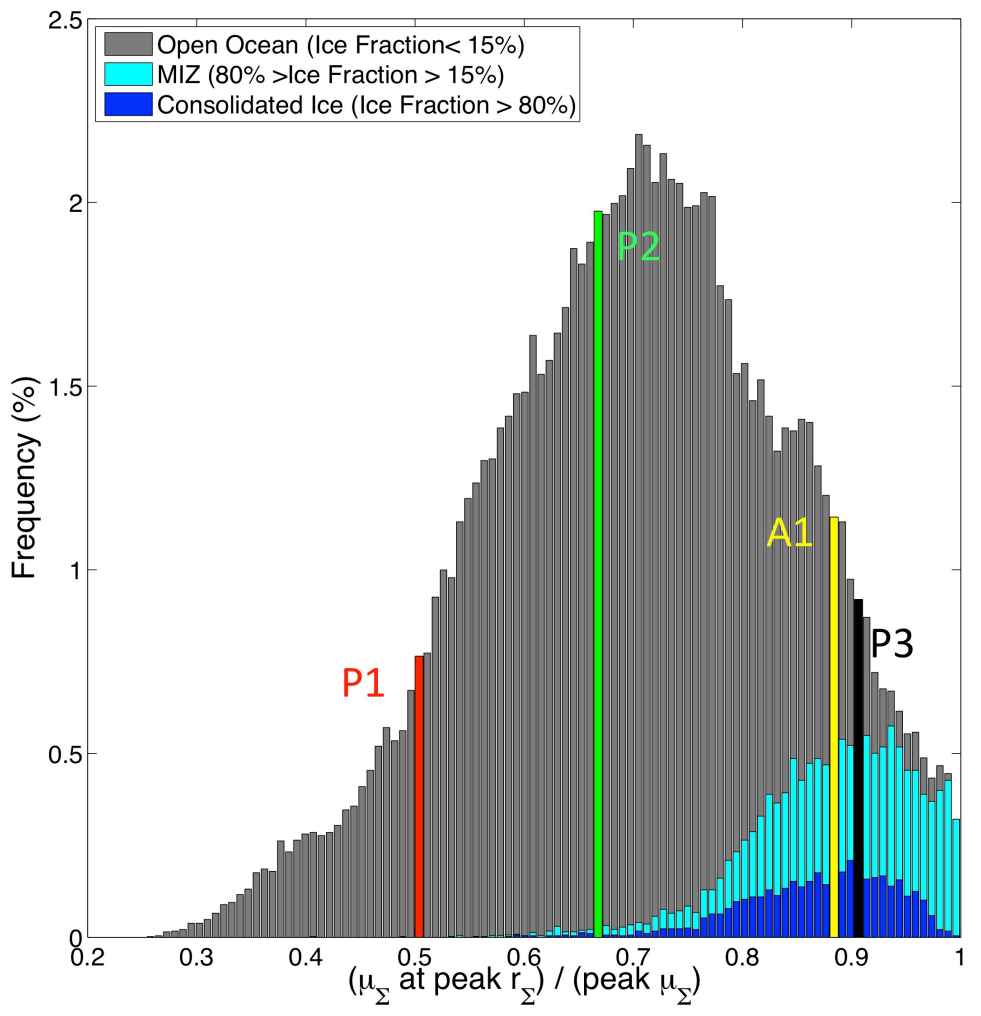

Figure 2-8: The frequency distribution of $\frac{\mu_{\Sigma} \text { at peak } r_{\Sigma}}{\operatorname{peak} \mu_{\Sigma}}$ for all 47,314 Southern Ocean grid cells plotted in Fig. 2-2a. The corresponding bins for the regional mean $\frac{\mu_{\Sigma} \mathrm{at} \text { peak } r_{\Sigma}}{\text { peak } \mu_{\Sigma}}$ of grid cells in P1 (red), P2 (green), P3 (black), and A1 (yellow) is delineated and color-coded for reference. Each bin is decomposed into the fraction of grid cells within the consolidated ice zone (blue), the $M I Z$ (cyan), and open-ocean (grey). Following [Stroeve et al. 2016], the $M I Z$ is defined as a mean annual ice fraction between $15 \%$ and $80 \%$, the consolidated ice zone as a mean annual fraction above $80 \%$, and open-ocean as a mean annual ice fraction below $15 \%$. 

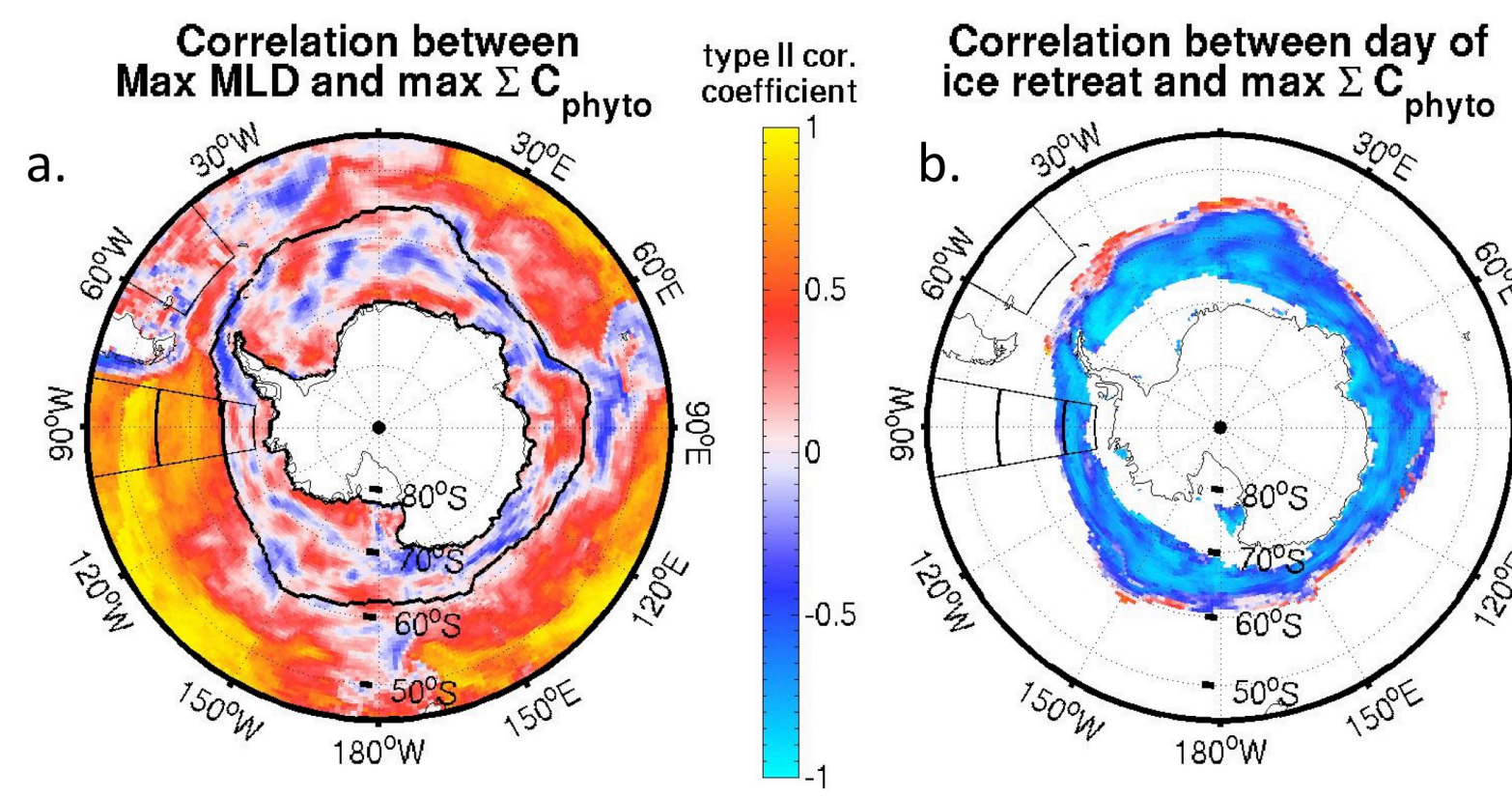

type II cor. coefficient
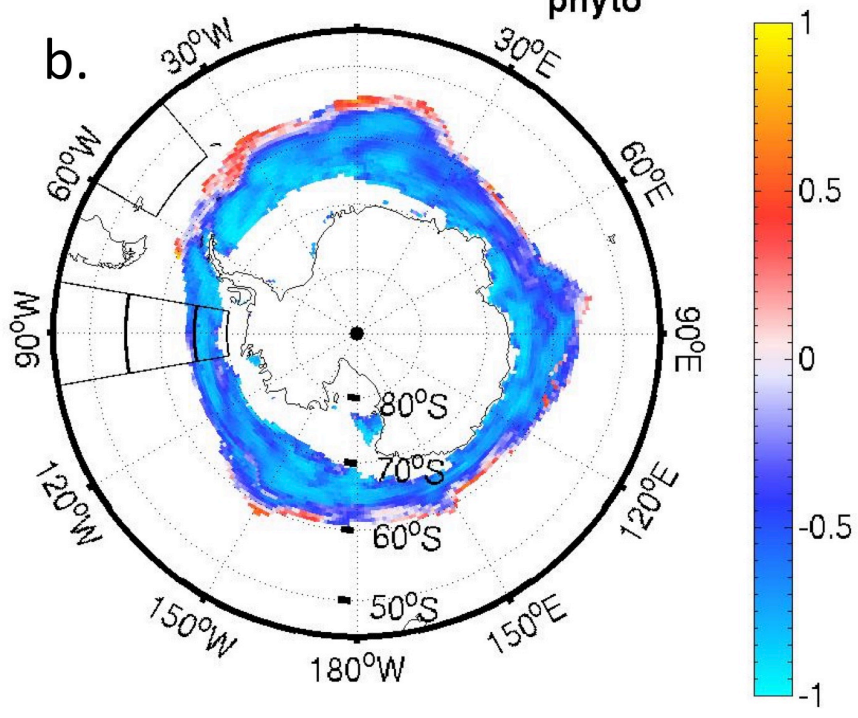

Figure 2-9: The simulated interannual correlation between (a) the maximum mixed layer depth and maximum $\Sigma C_{\text {phyto }}$ and (b) the day of sea-ice retreat (which co-varies with total annual ice volume) and maximum $\Sigma C_{\text {phyto }}$ across all years of the simulation computed independently at each model grid cell. The region that sees, on average, at least $15 \%$ mean annual fractional ice coverage is outlined in (a), along with regional bins P1, P2, P3, and A1 in both subplots. 
Chapter 3

\section{SIMULATED EDDY INDUCED BOTTOM-UP CONTROLS ON PHYTOPLANKTON GROWTH IN THE SOUTHERN OCEAN}




\begin{abstract}
Observational and modeling work point to the important role of mesoscale eddy processes in modulating biological productivity and ecosystem dynamics, but the relationship is subject to a great deal of regional and mechanistic variability which is, as of yet, poorly understood, particularly in the Southern Ocean. We examine the effects of Southern Ocean eddies in global, multi-year, eddy-resolving, 3-D ocean simulation of the Community Earth System Model. We first identify and track eddies in the simulation and validate their distribution and demographics against observed tracks. Next, we examine how simulated cyclones and anticyclones differentially modify iron availability, light limitation, and ultimately population specific division rates to understand how eddies regulate phytoplankton populations from the bottom-up. We use an eddy-centric, depth-averaged framework to explicitly examine the average dynamics of the entire phytoplankton population within an eddy. We find that eddy-induced Ekman pumping increases the upward advection of iron and improves population-averaged iron availability in anticyclones throughout the year. During the winter, in regions with deep background mixed layers, anticyclones induce anomalously deep mixed layers, which further supply new iron from depth via an increased upward mixing flux. This additional contribution, however, comes at the price of deteriorating light availability as biomass is distributed deeper in the water column. In turn, population-averaged specific division rates are predominately elevated in Southern Ocean anticyclones throughout most of the year, but decline in magnitude during the winter as background mixed layers deepen. In some regions wintertime mixed layers are deep enough that light limitation dominates over improved iron availability and division rates become depressed in anticyclones. This seasonal reversal is evident in the deep mixing Pacific sector of the Antarctic Circumpolar Current. The opposite is true in cyclones, which exhibit a consistently symmetric physical and biogeochemical response relative to anticyclones; cyclones predominately suppress division rates by reducing iron availability unless mixed layer anomalies are shallow enough to sufficiently relieve light limitation.
\end{abstract}

\title{
Key Points
}

- Mixed layer depths are anomalously deep in anticyclones and shallow in cyclones provided that the eddy is large and the background mixed layer is deep. Otherwise mixed layer depth anomalies are not necessarily aligned with polarity.

- Cyclones and anticyclones preferentially exhibit depressed and enhanced iron concentrations, respectively, regardless of the direction of their mixed layer depth anomaly.

- Eddy-induced Ekman pumping is the dominant vertical transport mechanism for iron, 
except in deep mixing winter regions, where anomalous entrainment via mixed layer modification is equally important, but generally in the same direction.

- Cyclones and anticyclones exhibit depressed and enhanced division rates, respectively, unless sufficiently deep wintertime background mixed layer depths allow the effects of light limitation to dominate those of iron limitation and the direction of division rate anomalies is briefly reversed.

\subsection{Introduction}

Globally, mesoscale scale phenomena account for roughly $25 \%$ of the surface ocean's area [Chaigneau et al. 2009] and 90\% of its kinetic energy [Ferrari and Wunsch 2008]. Observations [Doney et al. 2003; Frenger et al. 2018; Gaube et al. 2014a; Large 1998; McGillicuddy et al. 2007] and models [Anderson et al. 2011; Song et al. 2018] agree that mesoscale activity helps regulate spatial and temporal variability in biological productivity. The Southern Ocean is replete with mesoscale activity [Meredith 2016; Stevens and Killworth 1992] and a key contributor to the global "biological pump" [Hauck et al. 2015], but recent work (i.e. Dawson et al. [2018], Frenger et al. [2018], and Song et al. [2018]) has emphasized that the biophyisical relationship between Southern Ocean eddies and productivity is not fully understood. Mesoscale variability is thought to dominate spatio-temporal variability in phytoplankton distributions [Doney et al. 2003; Glover et al. 2018], meaning that a better understanding of eddy-mediated modifications to phytoplankton growth is critical to constraining Southern Ocean primary productivity, carbon storage [Marinov et al. 2008] and ultimately global climate dynamics [Chisholm 2000].

Closed rotating vortices, referred to as eddies, can force a biological response via many physical pathways [Gaube et al. 2014b; McGillicuddy 2016]. Eddies with different polarities (i.e. cyclonic vs. anticyclonic) are expected to induce a symmetric physical response. In the Southern Hemisphere cyclones rotate clockwise, dome isopycnals, and induce a cold sea surface temperature anomaly $\left(-S S T^{\prime}\right)$ and negative sea surface height anomaly $\left(-S S H^{\prime}\right)$. Anticyclones rotate counterclockwise, depress isopycnals, and induce warm $S S T^{\prime}$ and positive $S S H^{\prime}$. The biogeochemical reaction to this physical perturbation is complicated by various vertical and horizontal processes that compete to dominate the response.

Several processes are capable of modifying light and nutrient availability in the vertical direction. First, during eddy formation eddy pumping caused by the deformation of isopycnals can lead to nutrient upwelling in cyclones and downwelling in anticyclones [Falkowski et al. 1991]. The opposite response occurs during eddy decay [Franks et al. 1986]. Next, rotating eddy driven surface currents asymmetrically modify the relative wind stress and induce Ekman 
downwelling in cyclones and Ekman upwelling in anticyclones [Dewar and Flierl 1987; Gaube et al. 2014b]. The magnitude of eddy-induced Ekman pumping is thought to be smaller than that of eddy pumping, but eddy-induced Ekman pumping acts continuously, over the entire lifetime of an eddy [Gaube et al. 2013; McGillicuddy et al. 2007] whereas eddy pumping is a transient event isolated to eddy intensification and decay [McGillicuddy et al. 1998]. Finally, isopycnal displacement in conjunction with $S S T^{\prime}$ can modify local stratification and in turn the mixed layer depth within eddies relative to surrounding waters [Dufois et al. 2016; Song et al. 2018]. Cyclones, associated with cold $S S T^{\prime}$ and domed isopycnals, have been observed to exhibit shallow mixed layer depth anomalies throughout the Southern Ocean [Hausmann et al. 2017] as well as at lower latitudes [Dufois et al. 2014; Gaube et al. 2013]. Deep mixed layer depth anomalies are found in anticyclones. In the Southern Ocean, anomalous mixed layer depths can drive primary productivity in opposite directions by modifying deep iron availability [Carranza and Gille 2015] and population-averaged light availability, as phytoplankton are redistributed within the water column [Nelson and Smith 1991].

Eddies can also laterally advect biogeochemical tracers across a large-scale gradient by stirring water along their periphery as they rotate [Chelton et al. 2011a; Doney et al. 2003; Glover et al. 2018] and trapping water at their core as they propagate [Early et al. 2011; Flierl 1981; Lehahn et al. 2011]. Here we consider the potential contribution of lateral advective processes, but focus on vertical processes which are able to supply new nutrients to the euphotic zone and are more likely to stimulate/depress new production and carbon export. A more thorough discussion of horizontal transport mechanisms can be found in Frenger et al. [2018].

The existence of multiple pathways through which eddies can modify biology makes it difficult to diagnosis the precise cause of biogeochemical anomalies within them using observations alone. Observations have shown dramatic regional and seasonal variability in the correlation between $S S H^{\prime}$ (a proxy for eddies) and surface chlorophyll anomalies $\left([\mathrm{Chl}]_{S}^{\prime}\right.$ a proxy for biomass) [Gaube et al. 2014a, 2013; Song et al. 2018]. This variability implies that different mechanistic pathways can dominate at different times [Gaube et al. 2014b]. Unfortunately, remote sensing studies are limited in their ability to assess depth-integrated population dynamics, biological rate terms, and many in-situ biogeochemical processes (i.e. nutrient transport), often leaving uncertainty in the mechanisms driving these correlative relationships. Frenger et al. [2018], for instance, observed that anticyclones in the Antarctic Circumpolar Current (ACC) are associated with anomalously high $[C h l]_{S}$ in the shallow mixing summer months and anomalously low $[\mathrm{Chl}]_{S}$ in the deep mixing winter months, but could not fully constrain the underlying drivers. Depressed $[C h l]_{S}$ during winter in anticyclones, for example, may be explained by deeper mixing imposing harsher light limitation, intense eddy pumping (downwelling) suppressing the vertical nutrient supply, the dilution of the biomass profile, or lateral advection. Modeling work [Song et al. 2018] has gone on to propose that 60 
this seasonal flip hinges on the relative dominance of iron versus light limitation driven by changes in the background mixed layer depth, but does not consider coherent, rotating, eddy structures or explicitly examine how population-averaged division rates are modified.

Ambiguity in the cause of eddy-driven $C h l$ anomalies in eddies throughout the Southern Ocean could have profound implications on our understanding of larger biogeochemical cycling, depending on whether eddies are inducing/stifling new production or simply moving biomass around. To move forward, past work has underscored the need to leverage numerical simulations to directly address the effect of eddies on the entire biomass profile [Gaube et al. 2014b], quantify the source and consequence of anomalous nutrient/light limitation [Song et al. 2018], and explicitly resolve biological rate terms [Frenger et al. 2018]. The first step in better constraining the biogeochemical response to eddies is to understand how eddies modify their phytoplankton division rates from the bottom-up modulation of growth conditions. The combined effect of modified division rates and loss rates on net population growth is addressed and compared to the influence of physical transport mechanisms on the composite biomass anomaly in Chapter 4.

To do this, we employ an eddy-centric framework and weight depth-averaged variables by the biomass profile to accurately capture the mean state of the entire phytoplankton population within the boundaries of closed eddy features. Using the same simulation employed by Song et al. [2018], we first track individual eddies, identified as closed contours in $S S H^{\prime}$ [Faghmous et al. 2015] and compare these model-based eddy tracks to observations (3.3.1). Next we examine the dominant processes that could drive anomalous division rates in the Southern Ocean by regulating light [Fauchereau et al. 2011] and iron [Boyd 2002]. In 3.3.2 we address variability in light limitation imposed by anomalous mixed layer depths. In 3.3.3 we address variability in iron limitation and consider which sources dominate the anomalous supply of iron. Next we examine the combined effect of eddies on population averaged division rates (3.3.4). Finally, in addition to providing a complete Southern Ocean perspective, we highlight the intense seasonal variability seen in the ACC (3.3.5). Together, we provide a statistical context and step-by-step breakdown of the pathways by which simulated of eddies of variable size, season, region, and polarity modify division rates across the Southern Ocean (Fig. 3-1).

\subsection{Methods}

\subsubsection{Numerical simulation}

We analyze a global, eddy-resolving, numerical simulation integrated with the ocean [Smith et al. 2010], ice [Hunke and Lipscomb 2008] and biogeochemistry [Moore et al. 2013] components of the the Community Earth System Model (CESM1) [Hurrell et al. 2013], forced with 
atmospheric data from the Coordinated Ocean_ice Reference Experiment (CORE I) "normal year" [Griffies et al. 2009; Large and Yeager 2004]. This simulation was run for 5-years after initialization (see Harrison et al. [2018] for details on initialization), and model output was saved as 5-day means.

\section{Physics}

The physical oceans component is based on the Parallel Ocean Program version 2 (POP) [Smith et al. 2010] and was integrated on a global tri-pole grid with nominal horizontal spacing of $0.1^{\circ}$ and 62 vertical levels. Vertical resolution is $10 \mathrm{~m}$ over the euphotic zone $(0-150 \mathrm{~m})$ and then decreases with depth. High spatial resolution permits mesoscale dynamics and the prognostic development of ocean eddies [Harrison et al. 2018; Song et al. 2018]. Mesoscale variability compares well to Aviso satellite products and captures the intense variability observed in the Southern Ocean [Harrison et al. 2018]. A validation of eddy demographics with observed eddy tracks is presented here in 3.3.1.

Vertical mixing is based on the K-Profile Parameterization (KPP) developed by Large et al. [1994]. The KPP scheme has been shown to capture globally observed seasonal variability in mixed layer depths within 10m [Moore et al. 2013]. Historically, however, climate models have had trouble replicating winter mixed layer depths in the Southern Ocean [Downes et al. 2015; Sallée et al. 2013]. Coarser resolutions of CESM1, have underestimated deep winter mixing in by 100s of meters [Moore et al. 2013; Weijer et al. 2011]. The high resolution integration employed here, however, has been shown to capture deeper, more realistic winter mixed layers and improved spatial patterns throughout the Southern Ocean [Harrison et al. 2018]. Note that while the KPP scheme is employed in both the high and low resolution simulations, it is sensitive to the changes to lateral mixing experienced in the high resolution simulation. While biases still exist, the mean simulated climatologic winter mixed layer depth is over 300 meters across much of the ACC, particularly in the South Pacific and South Indian Oceans. This compares favorably with observations of 300-400m average mixed layer depths in winter throughout the same regions [Buongiorno Nardelli et al. 2017].

Sea ice is treated using the CICE4 component [Hunke and Lipscomb 2008]. The ice model does not sequester iron or resolve biogeochemistry. All atmospheric dust deposition over sea ice is deposited directly into the surface water.

\section{Biogeochemistry}

Biogeochemistry is treated with the Biogeochemical Element Cycle (BEC) model [Moore et al. 2013]. Global solutions, integrated at a coarser resolution, have been widely validated against global data sets and shown to capture basin-scale spatial distributions in production, nutrient 
and chlorophyll concentrations [Doney et al. 2009; Moore et al. 2001, 2004, 2013] in addition to key aspects of oceanic iron [Moore and Braucher 2008] and carbon cycling [Lima et al. 2014; Long et al. 2013; Moore et al. 2013]. More recently, this high resolution run has been shown to compare favorably to global chlorophyll distributions [Harrison et al. 2018] and match the observed Southern Ocean mesoscale variability in the correlation between $[C h l]_{S}^{\prime}$ and $S S H^{\prime}$ [Song et al. 2018].

BEC features a single class of zooplankton and three classes phytoplankton: diatoms, small phytoplankton, and diazotrophs. Phytoplankton carbon biomass, $C_{\text {phyto }}(\mathrm{mmol} C)$, is resolved independently for each phytoplankton pool (phyto) and tracked in terms of grid cell concentration, $\left[C_{\text {phyto }}\right]\left(\frac{m m o l C}{m^{3}}\right)$. Class specific phytoplankton net population growth $\left(\frac{d\left[C_{p h y t o}\right]}{d t}\right)$ is governed by a photosynthetic net primary productivity term, $P_{\text {phyto }}\left(\frac{m m o l C}{m^{3} d}\right)$, and opposed by a loss term, $L_{\text {phyto }}\left(\frac{m m o l C}{m^{3} d}\right)$, such that

$$
\frac{d\left[C_{\text {phyto }}\right]}{d t}=P_{\text {phyto }}-\text { Loss }_{\text {phyto }} .
$$

Loss $_{\text {phyto }}$ is composed of a nonlinear grazing, linear mortality, and quadratic mortality/ aggregation term.

$P_{\text {phyto }}$ is equal to a volumetric specific photosynthetic division rate, $\mu_{\text {phyto }}\left(d^{-1}\right)$, multiplied by the biomass concentration $\left(P_{\text {phyto }}=\mu_{\text {phyto }} *\left[C_{\text {phyto }}\right]\right)$. This division rate is subject to temperature dependence $\left(L^{T}\right)$, multi-nutrient $(N, P, S i, F e)$ limitation $\left(L^{N}\right)$ and light availability $\left(L_{\text {phyto }}^{I_{\text {par }}}\right)$ such that,

$$
\mu_{\text {phyto }}=\mu_{\text {phyto }}^{\max } * L^{T} * L_{\text {phyto }}^{N} * L_{\text {phyto }}^{I_{\text {par }}},
$$

where $\mu_{\text {phyto }}^{\max }$ is the maximum class-specific, volumetric-specific division rate. Unitless limitation terms vary from $0-1$ and scale the maximum division rate. In turn, lower values translate to greater stress. The model and observations agree that Southern Ocean productivity is primarily limited by light [Fauchereau et al. 2011] and iron [Boyd 2002]. Note that CESM1-BEC is able to reproduce large High Nitrate Low Chlorophyll (HNLC) regions in the Southern Ocean, subarctic and equatorial Pacific [Harrison et al. 2018; Moore and Braucher 2008; Moore et al. 2004].

Light limitation is computed following a modified form of the growth model developed by Geider et al. [1998] and is functionally dependent on photosynthetically available radiation, the most constraining nutrient limitation term, and a dynamic $C h l$ to $C$ ratio. Class specific nutrient limitation terms $\left(L_{\text {phyto }}^{F e}, L_{\text {phyto }}^{P}, L_{\text {phyto }}^{N}, L_{\text {phyto }}^{S i}\right)$ vary as a Holling-III nonlinear function of the available nutrient concentration and the class-specific half saturation coefficient. Multinutrient limitation is treated following Liebig's Law of the Minimum [van der Ploeg et al. 1999] such that the maximum specific division rate $\left(\mu_{\text {phyto }}^{\max }\right)$ is only scaled by the most limiting 
nutrient limitation term. Light and nutrient limitation, however, are co-limiting.

Iron is supplied to the ocean via atmospheric and benthic sedimentary sources following Moore and Braucher [2008]. The physical transport of dissolved inorganic iron $(F e)$ is dominated by fluxes $\left(\mu \mathrm{mol} / \mathrm{m}^{2} / \mathrm{s}\right)$ from horizontal advection $\left(U_{F e}, V_{F e}\right)$, vertical advection $\left(W_{F e}\right)$, and vertical diabatic mixing $\left(M i x_{F e}\right)$. All flux terms are averaged over the 5 day time step over which model output is saved. Vertical fluxes are positive in the upward direction.

\subsubsection{Description of diagnostic variables}

\section{Biomass-weighted, depth averaged variables}

Throughout the Southern Ocean, surface biomass is not always well correlated with the depthintegrated biomass inventory [Rohr et al. 2017]. Rather than focusing on surface concentrations (as done in Frenger et al. [2018], Gaube et al. [2014b], and Misumi et al. [2014], etc.), we express biogeochemically relevant variables as biomass-weighted depth averages to capture the complete biological response to eddy perturbation. In the context of strictly biological variables (i.e. limitation terms and division rates), this is simply a depth-averaged population mean. In the context of environmental variables (i.e. iron and iron supply rates) this represents the mean environmental state of the population. By considering the entire depth averaged phytoplankton population results are sensitive to variability in community composition, profile distribution, dilution during deep mixing [Behrenfeld et al. 2013; Rohr et al. 2017], biomass that may exist below a shallow mixed layer [Behrenfeld et al. 2013], and the possibility of nonuniform biomass profiles or subsurface maxima [McGillicuddy et al. 2007; Siegel et al. 1999]. Biomass-weighted, depth averaged variables are identified with the subscript $(\Sigma)$, and are computed as follows,

$$
\begin{aligned}
L_{\Sigma}^{F e} & =\sum_{z=0 m}^{\text {water column }} \sum_{\text {phyto }=\text { sp }, \text { diat }} L_{z, \text { phyto }}^{F e} \frac{\left[C_{\text {phyto }}\right]_{z} * h}{\text { Biomass Inventory }}, \\
L_{\Sigma}^{I_{P A R}} & =\sum_{z=0 m}^{\text {water column }} \sum_{\text {phyto }=\text { sp }, \text { diat }} L_{z, \text { phyto }}^{I_{P A R}} \frac{\left[C_{\text {phyto }}\right]_{z} * h}{\text { Biomass Inventory }}, \\
\mu_{\Sigma} & =\sum_{z=0 m}^{\text {water column }} \sum_{\text {phyto }=\text { sp }, \text { diat }} \mu_{z, \text { phyto }} \frac{\left[C_{\text {phyto }}\right]_{z} * h}{\text { Biomass Inventory }}, \\
{[F e]_{\Sigma} } & =\sum_{z=0 m} \sum_{\text {water column }}[F e]_{z, \text { phyto }} \frac{\left[C_{\text {phyto }}\right]_{z} * h}{\text { Biomass Inventory } y},
\end{aligned}
$$

where $C_{\text {phyto,z }}$ is the class specific biomass concentration at a given grid cell and depth 
$\left(\frac{m m o l}{m^{3}}\right), h$ is the height of the grid cell $(m)$, and the Biomass Inventory is the depthintegrated sum of diatom and small phytoplankton biomass $\left(\frac{m m o l}{m^{2}}\right)$. Note that only the two regionally dominant phytoplankton pools are considered, diatoms (diat) and small phytoplankton $(s p)$. Iron concentrations only include the dissolved inorganic pool because it is the dominant form of iron and including other pools did not change the results.

Biomass-weighted, depth averaged iron supply rates $\left(\mu \mathrm{mol} / \mathrm{m}^{3} / \mathrm{s}\right)$ capture the average rate at which iron is supplied to all phytoplankton across the water column by either vertical mixing or vertical advection. They are computed by averaging the vertical flux divergence at each grid cell across the water column and weighting by the fraction of the biomass inventory contained at each depth as follows;

$$
\frac{d[F e]}{d t}_{M i x, \Sigma}=\sum_{z=0 m}^{\text {water column }} \sum_{\text {phyto=sp,diat }}\left(M i x_{F e, \text { bottom }}-M i x_{F e, t o p}\right) \frac{1}{h} \frac{\left[C_{\text {phyto }}\right]_{z} * h}{\text { Biomass Inventory }}
$$

$$
\frac{d[F e]}{d t}_{W, \Sigma}=\sum_{z=0 m}^{\text {water column }} \sum_{\text {phyto }=\text { sp }, \text { diat }}\left(W_{F e, \text { bottom }}-W_{F e, \text { top }}\right) \frac{1}{h} \frac{\left[C_{\text {phyto }}\right] \text { z } * h}{\text { Biomass Inventory }} .
$$

Iron supply rates are defined as positive if there is a net flux into the grid cell. This framework captures the degree of iron that is transported to where phytoplankton actually are and ensures results are not biased by iron supplied to grid cells without any biomass. The total time-integrated supply of iron from either vertical mixing or advection is computed by integrating the supply rate between eddy formation and any given realization during its lifetime $\left(\int_{\text {formation }}^{\text {realization }} \frac{d[\mathrm{Fe}]}{d t} \operatorname{Mix}_{\mathrm{\Sigma}} \mathrm{\Sigma} d t\right.$, etc $)$.

\section{Horizontal iron transport potential}

It is difficult to attribute the precise contribution of lateral advection to the iron anomaly field, particularly as iron is simultaneously upwelled, consumed, and regenerated, but following Frenger et al. [2018] we can provide an estimate based on environmental conditions and eddy characteristics. The size and direction of anomalies that can be created by lateral advection is bound by the size and direction of the horizontal iron gradient as well as the size, polarity, and propagation of the eddy. In turn we can estimate the potential for an anomaly to arise exclusively from either Trapping or Stirring by computing how an isolated eddy with known properties would perturb the climatological gradient (see 3.2.4). Briefly, the trapping potential represents the anomaly that would be created if the source water was simply transported 
across the gradient as it propagates. The stirring potential represents the anomaly that could be created from stirring across the meridional gradient while propagating westward relative to the mean flow. See Frenger et al. [2018] for a more detailed description.

\section{Eddy induced Ekman pumping}

The velocity of eddy-induced Ekman pumping $(\mathrm{m} / \mathrm{s})$ is estimated diagnostically from the curl of the relative surface stress $(\nabla \times \tau)$ as follows,

$$
\Omega_{E k}=\frac{\nabla \times \tau}{\rho_{o} f}
$$

where $\tau$ is the relative surface stress between the wind and surface currents, $\rho_{o}$ is the density of water, and $\mathrm{f}$ is the Coriolis parameter $(f=2 \Omega \cos \theta)$ at latitude $\theta$ with a global rotation rate $\Omega$. Within eddies, the curl of the relative surface stress and thus eddy-induced Ekman pumping is modified directly by rotating surface currents [Gaube et al. 2014a; McGillicuddy 2016]. In nature, eddy-induced Ekman pumping is further modified eddy-induced SST anomalies which in turn modify wind speeds [Chelton et al. 2004; O'Neill et al. 2010] and a nonlinear contribution to vertical transport induced by the vorticity gradient. These two processes are not resolved in this simulation, however, the $S S T$ contribution should act in the same direction as the contribution from rotating surface currents, and the nonlinear contribution is likely to average out across the sub-mesoscale [Gaube et al. 2014b].

\subsubsection{Depth extrapolation}

Some model output is extrapolated at depth as storage limitations prevent saving the full-

depth profiles of all prognostic tracers. In this simulation biomass $\left(\left[C_{\text {phyto }}\right]_{z}\right)$, division rates $\left(\mu_{\text {phyto,z }}\right)$, and limitation terms $\left(L_{\text {phyto,z }}^{F e}\right.$ and $\left.L_{\text {phyto,z }}^{I_{p a r}}\right)$ were archived to $150 \mathrm{~m}$. Dissolved iron $\left([\mathrm{Fe}]_{z}\right)$ and all component fluxes were saved to the ocean floor.

If the the mixed layer depth $(M L D)$ is shallower than $150 \mathrm{~m}$ it is reasonable to assume that biomass below $150 \mathrm{~m}$ cannot access the euphotic depth and is likely negligible. In turn, we assume biomass profiles drop to zero below $150 \mathrm{~m}$. During the winter, however, deep mixing can penetrate well below $150 \mathrm{~m}$ leading to the existence of biomass beneath what has been explicitly saved. In order to diagnostically characterize the complete biological response during deep mixing events $(M L D>150 \mathrm{~m})$, all relevant profiles are linearly extrapolated to the $M L D$. Biomass and it's associated limitation and rate terms are assumed to drop to zero immediately below the $M L D$. A linear extrapolation is reasonable considering that biomass profiles have been observed to be well-approximated by a uniform distribution in deep mixed layers below the euphotic zone specifically in the Southern Ocean [Uitz et al. 2006], as well 
as in the North Atlantic [Behrenfeld et al. 2013; Boss and Behrenfeld 2010]. Light limitation is expected to decay exponentially [Geider et al. 1998] but should already be asymptotically approaching it's minimum $\left(L^{I_{p a r}}=0\right)$ prior to linear extrapolation below $150 \mathrm{~m}$.

\subsubsection{Anomaly and Climatology Fields}

Anomaly fields for each relevant variable $\left(v a r^{\prime}\right)$ are strictly spatial and are computed by removing the mean field $(\overline{v a r})$ from the raw field (var), such that,

$$
v a r^{\prime}=v a r-\overline{v a r}
$$

Mean fields were created by smoothing the raw fields with a $2 \mathrm{D}$ low pass loess filter. Following Gaube et al. [2014b], a half power cutoff of $20^{\circ}$ in longitude and $10^{\circ}$ in latitude was used to create the sea surface height anomaly $\left(S S H^{\prime}\right)$, and a $6^{\circ} \times 6^{\circ}$ filter was used for all biogeochemically relevant fields to best target mesoscale variability. Additional filters (notably a $20^{\circ} \mathrm{x} 10^{\circ}$ version for biogeochemical fields) were tested but did not qualitatively effect the nature of the results. Three-dimensional anomaly fields were computed independently at each depth.

Background climatology fields $\left(\overline{v a r}_{C l i m}\right)$ were computed by averaging over the 5-year model run at each time step (5-days). To help remove lingering mesoscale variability left from the relatively short model run, climatologies were smoothed with a 30-day moving average in time and a $6^{\circ} \times 6^{\circ}$ loess filter in space. Filters were chosen carefully to retain large-scale seasonal and geographic variability. Normalized fields $\left(v a r^{\prime \prime}\right)$ were computed by dividing anomaly fields by the corresponding climatology such that,

$$
\operatorname{var}^{\prime \prime}=\frac{\operatorname{var}^{\prime}}{\overline{v a r}_{C l i m}}
$$

\subsubsection{Eddy identification and tracking}

Unlike much of the prior correlative work (e.g. Gaube et al. [2014b], Song et al. [2018]) our eddy-centric approach specifically operates on coherent mesoscale structures rather than individual grid cells that may have a strong $S S H^{\prime}$ signal but are less likely to be enclosed by a rotating vortex. Here, eddies are identified as closed contours in the sea surface height anomaly $\left(S S H^{\prime}\right)$ field independently at each time step using the parameter-less algorithm originally developed by Chelton et al. [2011b] and adapted by Faghmous et al. [2015]. Using closed contours in the $S S H^{\prime}$ field to isolate eddies reduces the probability of including spurious, non-rotating mesoscale features and helps isolate the mechanisms that are unique to actual eddies. Song et al. [2018], for instance, estimate that 33\% of analyzed data are not enclosed by an $S S H^{\prime}$ contour at all. Eddies here are explicitly defined as the outermost closed contour 
surrounding a single extrema. Excluding eddies with multiple local maxima or minima reduces the problem of mistakenly merging nearby eddies associated with the Chelton et al. [2011b] methodology. For cyclones this extrema is a local minimum and for anticyclones it is a local maximum. The amplitude is defined as the difference between the extrema and outermost contour. The speed-based eddy radius $\left(L_{S}\right)$ is approximated as the radius of a circle with an area equal to that enclosed by the $S S H^{\prime}$ contour around which the average geostrophic speed is maximized [Faghmous et al. 2015].

This geometric implementation of satellite-based altimetry to identify eddies offers a large improvement over older methods that employed proxy variables such as ocean color [Pegau et al. 2002] or sea surface temperature [Dong et al. 2011], which are correlated with eddies but influenced by a number of other mechanisms. Note that altimetric data can also be used to identify eddies on a physical basis. The most common physical basis for identifying eddies is the Okubo-Weiss (W) parameter which relates the degree of rotation quantified by the relative vorticity to the degree of strain [Okubo 1970; Weiss 1991]. The problem with these sort of methods is that they require an expert defined, often regionally-specific, threshold [Isern-Fontanet et al. 2003], and suffer from the introduction of addition noise as the ' $\mathrm{W}$ ' parameter is derived from the derivative of the velocity field [Azevedo Correia de Souza et al. 2011]. Geometric methods, such as the Faghmous et al. [2015] method employed here introduce less noise and notably do not require any expert defined thresholding, making them more suitable for larger spatial and temporal scales [Azevedo Correia de Souza et al. 2011]. Newer techniques have sought to explicitly identify coherent lagranigian structures, which isolate eddies that necessarily trap fluid at their core [Beron-Vera et al. 2008; Prants et al. 2011; Samelson 2013]. These methods likely reduce the number of type II errors but are much more computationally expensive. While it is true that the Faghmous et al. [2015] methodology is susceptible to misidentifying meanders, or other mesoscale features, as eddies, it is computationally efficient, spatially robust in that it eliminates the need for regionally specific thresholds, and has been validated with a composite analysis of identified features as well as a direct comparison to eddies manually identified by experts [Faghmous et al. 2015]. We conducted extensive additional testing to isolate "true" eddies by further filtering for properties such as shape, size, and propagation distance, however, due to the dramatic physical variability amongst "true" eddies [Fu et al. 2010], any filter is apt to remove both spurious and real features. Ultimately, although some strict filtering produced larger anomalies, no filtering scheme qualitatively altered the nature of the results.

After identification, eddies are associated between time-steps to create a portfolio of eddy tracks. Eddies at time-step $\mathrm{t}$ are associated with the closest eddy realization at time-step $\mathrm{t}+1$ within a predefined search radius, bounded by the maximum theoretical distance the eddy could have traveled between time $\mathrm{t}$ and $\mathrm{t}+1$ [Faghmous et al. 2015]. Once the closest 
realization has been identified at time $t+1$ it is checked to confirm both features are similar in size and amplitude. This process is iterated until no eddy is found within the search window for more than two time steps. Eddy tracks that last less than 60 days or never cross south of $40^{\circ} \mathrm{S}$ were not considered. The Weddell Sea region, where the simulation develops a large polynya with unrealistically deep mixed layer depths, was excluded from consideration. No further filtering was imposed unless explicitly mentioned.

Each simulated eddy track is in turn composed of a time series of eddy "realizations" identified at 5 -day intervals in the observations. Note that realizations younger than 60 days from tracks that are longer than 60 days are included in the analysis to ensure we consider the formation phase of eddies. A simulated eddy track with a lifetime of 100 days, for example, contains 20 saved realizations. Eddy-averaged values were created for all realizations by co-locating each relevant variable with the footprint of a given eddy realization defined by the closed $S S H^{\prime}$ contours identified in the tracking algorithm. By spatially averaging biomass-weighted depth averaged fields, values are representative of the mean state of all phytoplankton within an eddy realization. Eddy-averaged values from all realizations contribute independently to our analysis.

In 3.3.1 the distribution and demographics of simulated eddy tracks are compared to observed eddy tracks originally reported by Chelton et al. [2011b] and more recently updated by Chelton and Schlax [2016] using improved altimeter observation described by Pujol et al. [2016] and a variation of the tracking methodology described by Williams et al. [2011]. Observational realizations are recorded at daily intervals rather than the 5-day time step required in the simulation.

\subsubsection{Eddy Subsets}

To better understand how different types of eddies perturb their environment, eddies are often binned on the basis of their size, background environment, or geographic location. Eddies are classified as large if their radius is greater than $50 \mathrm{~km}$ and their amplitude is greater than $5 \mathrm{~cm}$ $\left(L_{s}>50 \mathrm{~km}, A m p .>5 \mathrm{~cm}\right)$ or small if their radius is smaller than $50 \mathrm{~km}$ and their amplitude is smaller than $5 \mathrm{~cm}$. Environmentally, eddies with a background climatologic mixed layer depth

$\left(\overline{M L D}_{\text {Clim }}\right)$ deeper or shallower than $100 \mathrm{~m}$ are defined as deep mixing or shallow mixing respectively. Geographically, the ACC is dynamically defined as between the $-20 \mathrm{~cm}$ and $-80 \mathrm{~cm}$ contours in the $S S H$ climatology $\left(\overline{S S H}_{C l i m}\right)$ following [Frenger et al. 2018]). The deepest mixing, Pacific sector of the ACC is further constrained between $80^{\circ} \mathrm{W}$ and $180^{\circ} \mathrm{W}$. 


\subsection{Results}

\subsubsection{Simulated and observed eddy track demographics}

A similar number of Southern Ocean eddies are identified in the simulated and observed eddy tracks each year. 288,120 individual realizations were found over the 5 year model run at 5-day intervals compared to 5,696,000 realizations found over 22 year observation record at daily intervals. On an average day, this translates to roughly 789 eddy realizations in the simulation and 708 in the observations. These realizations comprise 13,414 unique eddy tracks in the simulation (or 2,682 per year) and 39,912 tracks in the observational record (or 1,814 per year). Although there are fewer unique eddy tracks per year in the observations, observed eddies tend to last longer, with an average lifespan of 147 days compared to 109 days in simulated eddies. This discrepancy may be explained by the coarser time step on which simulated eddies were tracked (5 days vs. 1 days), which increases the likelihood of misidentifying a single long lived track as two shorter lived tracks. For instance, tracking observed eddies at a weekly resolution as been shown to statistically decrease the lifetime of identified lifetime of eddies (http://wombat.coas.oregonstate.edu/eddies/Pino_figures_ for_Daily_Eddy_Dataset_Jan_1993-Apr_2015.pdf).

Variability in the location, timing, and size of simulated and observed eddies are compared in Fig. 3-2. The zonal distribution of eddies compares favorably between simulation and observation (Fig. 3-2a), however, meridionally there is a bias for increased eddy activity at high latitudes, often below sea ice, in the simulation (Fig. 3-2b). Seasonally, eddies are more likely to occur in the summer in the observations than the simulation (Fig. $\mathbf{3 - 2 b}$ ). Observed eddies tend to be larger in radius (Fig. 3-3e) and amplitude (Fig. 3-3f) than simulated eddies. Note that simulated eddies south of $60^{\circ} \mathrm{S}$, where the model overestimates the number of eddies, tend to be much smaller $\left(L_{S}=45 \mathrm{~km} ;\right.$ Amplitude $\left.=4.2 \mathrm{~cm}\right)$ than those at lower latitudes $\left(L_{S}=66 \mathrm{~km} ; A m p=7.5 \mathrm{~cm}\right)$ where the rossby radius is much larger, likely accounting for the bias in small simulated eddies. Despite this bias, the distribution of large eddies is generally consistent between the simulation and observations (Fig. $\mathbf{3 - 3} \mathbf{g}, \mathbf{h}$ ). For this reason, large eddies are often considered independently (see 3.2.6).

Maps of the geographic distribution of simulated eddy tracks during each season are provided in Fig. 3-3. Maps of the distributions of observed tracks can be found at http: //wombat.coas.oregonstate.edu/eddies/index.html [Chelton and Schlax 2016]. Simulated biomass distributions are included to provide context for where simulated eddies travel relative to regions of high or low biomass but eddy-induced anomalous biomass is address in

\section{Chapter 4.}




\subsubsection{Mixing and light limitation}

\section{Anomalous mixed layer depth anomalies}

Simulated eddies are likely to deform the mixed layer depth $(M L D)$ in the theoretically expected direction if they are large and/or subject to deep background mixing (Tab. 3.1). Within the subset of large, deep mixing eddies, $72 \%$ of cyclones exhibit shallower mixed layers than surrounding waters $\left(-M L D^{\prime}\right)$ while $78 \%$ of anticyclones exhibit deeper mixed layers $\left(+M L D^{\prime}\right)$. On the other hand, the direction of $M L D^{\prime}$ in small, shallow mixing eddies, often contradicts theory (i.e. $+M L D^{\prime}$ in cyclones), but the magnitude of these anomalies $(\sim 4 m)$ is on average one sixth the size of $M L D^{\prime}$ in large, deep mixing eddies $(\sim 28 m)$. As both eddy size and background mixing increase, so too does the magnitude of $M L D^{\prime}$ and the likelihood that it is in the theoretically expected direction (Fig. 3-4a - c). See Table $\mathbf{3 . 1}$ for detailed statistics.

The signature of this variability is clearly evident in the spatial and seasonal distribution of $M L D^{\prime}$ (see Fig. 3-5). During the weak mixing summer months (J,F,M) $M L D^{\prime}$ is small and inconsistent in direction (Fig. 3-5g - i). During the deep mixing winter months (J,A,S) $M L D^{\prime}$ is on average $\sim 8$ times larger in magnitude than in summer (or $\sim 3 \mathrm{x}$ when normalized by the climatology) and much more likely to be in the theoretically expected direction (Fig. 3-5a - c). This is particularly evident along the wintertime ACC, where background mixing is very deep. North of the ACC, in the quiescent subtropical basins, the magnitude of anomalies are reduced. South of the ACC, in the Marginal Ice Zone where eddy size is statistically smaller and background mixing is shallow, the direction of $M L D^{\prime}$ is the most consistently contradictory to theory.

\section{Anomalous light limitation}

Anomalous light limitation $\left(L_{\Sigma}^{I_{P A R^{\prime}}}\right)$ is controlled by eddy-induced changes in vertical mixing, and to a lesser extent, self-shading. $L_{\Sigma}^{I_{P A R}}$ is anti-correlated with both $M L D^{\prime}(r=-.53)$, which increases light stress as the population is mixed into poorly-lit deep water, as well as the anomalous biomass inventory $(r=-.29)$, which can increase light stress via self shading. The anti-correlation between $\left(L_{\Sigma}^{I_{P A R \prime}}\right)$ and $M L D^{\prime}$ is stronger because the relationship between $\left(L_{\Sigma}^{I_{P A R^{\prime}}}\right)$ and biomass is complicated by the direct effect of $\left(L_{\Sigma}^{I_{P A R^{\prime}}}\right)$ on division rates. Overall, $\left(L_{\Sigma}^{I_{P A R^{\prime}}}\right)$ maps well onto the distribution of $M L D^{\prime}$ (Fig. B-1, 3-5), particularly in large, deep mixing eddies where the anti-correlation between $\left(L_{\Sigma}^{I_{P A R^{\prime}}}\right)$ and $M L D^{\prime}$ is the strongest $(r=-.87)$. Within this subset, the population averaged light limitation anomaly is $11 \%$ higher (less stress) than climatologic values in cyclones and $12 \%$ lower (more stress) in anticyclones. This relationship is not as strong in shallow mixing waters where $\left(L_{\Sigma}^{I_{P A R} \prime}\right)$ is increasingly 
dominated by self-shading rather than the dilution of the biomass profile. The seasonal and regional distribution of $\left(L_{\Sigma}^{I_{P A R^{\prime}}}\right)$ is provided in Appendix B, Figure B-1 and additional statistics are provided in Table 3.1.

\subsubsection{Iron availability, limitation, and sources}

\section{Anomalous iron concentrations}

There is a clear discrepancy in the iron available to phytoplankton within simulated eddies; cyclones preferentially decrease the iron concentration available to phytoplankton populations within them $\left([\mathrm{Fe}]_{\Sigma}\right)$ while anticyclones increase it (Table 3.1). The magnitude of these anomalies is on average greater than $\sim 10 \%$ of co-located climatologic values and tends to increase with eddy size and the intensity of background mixing (Figs. 3-4e - g). Seasonal variability in the magnitude of $[F e]_{\Sigma}^{\prime}$ is not as strong as seen in the simulated mixed layer

depth anomalies (Figs. 3-5 \& 3-6), with the magnitude of $[\mathrm{Fe}]_{\Sigma}^{\prime}$ only $48 \%$ larger (or $5 \%$ if normalized by the climatology) in the winter than the summer. Geographically, there is also less variability in the direction of anomalies. The only notable exception occurs north of the $\mathrm{ACC}$ in the SE Pacific where only about half of anticyclones exhibit elevated $[\mathrm{Fe}]_{\Sigma}$ despite mostly deeper mixed layer anomalies. (Figs. 3-5 \& 3-6). The magnitude of these anomalies is, however, very low. Unlike $M L D^{\prime}$, heavy ice coverage does not systematically change the sign of $[\mathrm{Fe}]_{\Sigma}^{\prime}$. Note that the aolian deposition of iron above model sea-ice is passed straight through to the surface ocean.

\section{Anomalous iron Limitation}

Iron limitation $\left(L_{\Sigma}^{F e \prime}\right)$ is directly controlled by the ambient dissolved iron concentration, which is consistently the most limiting nutrient. Accordingly, the distribution of population averaged iron limitation anomalies $\left(L_{\Sigma}^{F e \prime}\right)$ maps very well onto the distribution of $[F e]_{\Sigma}^{\prime}($ Fig. B-2, 3-6), with iron limitation largely relieved in anticyclones and exacerbated in cyclones. The seasonal and regional distribution of $\left(L_{\Sigma}^{F e \prime}\right)$ is provided in Appendix B, Figure B-2 and additional statistics are provided in Table 3.1.

\section{Sources of anomalous iron}

Anomalous mixed layer depths and continuous eddy-induced Ekman pumping both preferentially enhance the vertical iron supply to phytoplankton inside of anticyclones and suppress it in cyclones (Fig. 3-8). The transient process of eddy pumping during formation acts in the opposite direction, however, does not play a dominant role here. Together, the anomalous iron derived from vertical transport is similar in magnitude to the potential contribution 
from lateral processes (Fig. 3-9), suggesting that a meaningful fraction of anomalous iron concentrations simulated in eddies is related to the availability of new iron from depth which is capable of fueling or suppressing new production.

Deeper mixed layers increase access to deep iron-rich water leading to an increase in the anomalous upward mixing flux of iron in eddies $\left(M i x_{F e}^{\prime}\right)$ as both background and anomalous mixed layer depths increase (Figs. 3-8a - d). In turn, the biomass-weighted depth averaged iron supply rate from vertical mixing $\left(\frac{d[\mathrm{Fe}]}{d t} \operatorname{Mix}, \Sigma\right)$ is elevated in the majority of anticyclones (68\%), particularly in the ACC $(81 \%)$ where $M L D$ anomalies are very deep. The ensuing anomalous population averaged iron availability $\left([F e]_{\Sigma}^{\prime}\right)$, however, in not very well correlated with $M L D^{\prime}(r=.14)$ or ${\frac{d[F e]^{\prime}}{d t}}_{M i x, \Sigma}(r=.15)$. In fact, the majority of anticyclones $(71 \%)$ with anomalously shallow mixed layers still contain more iron $\left(+[F e]_{\Sigma}^{\prime}\right)$ than the deeper mixing waters surrounding them and often exhibit an enhanced upward mixing flux of iron $\left(+M i x_{F e}^{\prime}\right)$ despite a shallower $M L D$ (Fig. 3-8d). It is clear that another mechanism is also modifying the vertical iron profile and ultimately the population averaged iron availability within eddies.

Eddy pumping, however, cannot help explain the distribution of $[F e]_{\Sigma}^{\prime}$. If eddy pumping dominated, anticyclones would experience downwelling that depresses the upward advection of iron for a transient period during formation and is followed by reduced iron concentrations. Instead anticyclones predominately experience enhanced upward advection of iron $\left(+W_{F e}^{\prime}\right)$ that is maintained throughout their lifetime (Fig. 3-8f) and coincident with elevated iron concentrations (Figs. 3-6, B-4). The opposite is true of cyclones (Fig. 3-8e). A small contribution from eddy pumping may explain the slight reduction in the magnitude of $W_{F e}^{\prime}$ apparent over the first month of eddy formation (Figs. 3-8e - f), but is not large enough to affect the sign of the anomaly.

Eddy-induced Ekman pumping, on the other hand, operates in a direction that is consistent with $W_{F e}^{\prime}$ and appears to dominate the anomalous vertical advection of iron in eddies. Anomalous vertical velocities induced by rotating surface currents $\left(\Omega_{E k}^{\prime}\right)$ are negative (downward) in $89 \%$ of cyclones and positive (upward) in $88 \%$ of anticyclones (Fig. B-5). The size and direction of $\Omega_{E k}^{\prime}$ is commensurate with anomalies in the total vertical velocity field (not shown), emphasizing the contribution of eddy-induced Ekman pumping to variability in vertical advection. As Ekman-induced vertical velocities increase, so too does the flux of iron via vertical advection (Figs. 3-8g - h). Together, Eddy-induced Ekman pumping appears to increase the rate at which vertical advection supplies iron to phytoplankton $\left(\frac{d[F e]}{d t}{ }_{W, \Sigma}\right)$ in $75 \%$ of anticyclones and decrease it in $74 \%$ of cyclones (Fig. B-5).

The bulk contribution of anomalous iron from vertical advection is on average larger than the contribution from vertical mixing. The total anomalous time-integrated supply of iron to phytoplankton in eddies from both vertical transport pathways is plotted in Fig. 3-9. Distributions only include large eddies in the $\underset{73}{\mathrm{ACC}}$ where $M L D$ anomalies are the most con- 
sistent with theory. In the average anticyclone vertical advection has cumulatively supplied $.015 \mu \mathrm{mol} / \mathrm{m}^{3}$ more iron to the grid cell of the average phytoplankton since formation than in surrounding waters. Vertical mixing, on the other hand, only supplies an extra $.005 \mu \mathrm{mol} / \mathrm{m}^{3}$ of anomalous iron, however, the relative contribution of vertical mixing increases when exclusively considering the deepest mixing winter months or Pacific sector of the ACC. The opposite is true of cyclones. For context, vertical processes collectively modify iron concentrations by about $20 \%$ of background levels $\left(\sim 0.10 \mu \mathrm{mol} / \mathrm{m}^{3}\right)$, and because background concentrations are below the modelled half saturation coefficient for diatoms $\left(\sim 0.15 \mu \mathrm{mol} / \mathrm{m}^{3}\right)$, the effect on iron limitation is more pronounced than in iron replete waters.

To confirm that anomalous vertical iron transport is not dwarfed by the horizontal advection of iron, we estimate the potential for anomalies to arise exclusively from lateral processes (see 3.2.2) and find that vertical and horizontal processes account for similar contributions to the anomalous iron concentrations. Estimates for the potential anomaly that could be created by both lateral Trapping and Stirring are included in Fig. 3-9 and operate in the same direction at a similar magnitude to vertical pathways. While these estimates are not part of the actual iron budget, it appears that anomalously high iron concentrations in anticyclones are composed of a roughly similar contribution of iron transported from depth as iron that was horizontally advected.

\subsubsection{Anomalous division rates}

Biomass-weighted, depth averaged division rates $\left(\mu_{\Sigma}\right)$ are widely depressed in cyclones and elevated in anticyclones, with anomalies on average $6.5 \%$ the size of co-located climatologies (Fig. 3-7; Tab. 3.1). Variability in anomalous division rates is predominately associated with the depth of the background mixed layer $\left(\overline{M L D}_{C l i m}\right)$ which modifies the balance between the competing effects of light and iron limitation. When background mixed layers are shallow eddy-induced $M L D$ anomalies are small, plankton are largely iron limited, and anomalous division rates are primarily modified by iron transported via eddy-induced Ekman pumping. As background mixed layers deepen the size of eddy-induced $M L D$ anomalies increases, plankton become increasingly light limited, and the influence of iron is dampened by the competing effect of increasing light limitation, reducing the magnitude of anomalous division rates. Eventually, once background mixing is deep enough $(\sim 150 \mathrm{~m}), \mu_{\Sigma}^{\prime}$ flips sign and division rates become more likely to increase in cyclones and decrease in anticyclones. See Fig. 3-4k.

The prevalence of this reversal in the direction of $\mu_{\Sigma}^{\prime}$ is evident seasonally when the frequency of elevated division rates in iron-rich anticyclones drops from $85 \%$ to $67 \%$ between summer and winter months (Fig. 3-7). During the summer, mixed layers are more likely to

be shallow and anomalous division rates are not well correlated with anomalous light limita- 
tion $(r=-.17)$, but during the deeper mixing winter months the strength of this correlation increases substantially $(r=.64)$, leading to more anticyclones with depressed division rates dominated by harsh light limitation. Geographically, this is most evident in the Pacific sector $\left(180^{\circ} \mathrm{W}<\mathrm{LON}<80^{\circ} \mathrm{W}\right)$ of the ACC, where deep winter mixing is correlated with depressed division rates in anticyclones and elevated division rates in cyclones.

\subsubsection{Seasonal variability in deep mixing ACC eddies}

Large eddies within the deep mixing Pacific sector of the ACC (see 3.2.6) exhibit a striking seasonal cycle. In Fig. 3-10 the seasonal progression of anomalous light and iron limitation, vertical iron transport, division rates and biomass is plotted both as both depth-averaged population means (upper axis) as well as fully resolved depth profiles that are not weighted by biomass (lower axis). Biomass anomalies $\left(\left[C_{p h y t o}\right]^{\prime}\right)$ are not discussed in detail (see instead, Ch. 4) but are included here to better understand how the redistribution of biomass influences other biomass-weighted variables.

Eddies modify the vertical advective flux of iron $\left(W_{F e}^{\prime}\right)$ throughout the year (Fig. 3-10a, b) but only induce a strong vertical mixing flux $\left(M i x_{F e}^{\prime}\right)$ during the winter (Fig. 3-10c, d) when $M L D^{\prime}$ is the largest. In turn, the iron limitation anomaly $\left(L_{\Sigma}^{F e \prime}\right)$ is supported throughout the year and across the water column by eddy-induced Ekman pumping, but maximized in the winter with the additional contribution from vertical mixing (Fig. 3-10e, f). Population averaged light limitation $\left(L_{\Sigma}^{I_{P A R} \prime}\right)$ is only meaningfully impacted during the winter and spring (Fig. 3-10g, h). During the winter, light stress increases (i.e. $L_{\Sigma}^{I_{P A R}}$ is more negative) in anticyclones as deeper mixing dilutes biomass across poorly-lit deep water (Fig. 3-10j). During the spring, well-lit surface biomass concentrations increase, but increased light limitation is sustained through increased self-shading.

Together, the role of iron dominates for much of the year, fueling anomalously high division rates $\left(\mu^{\prime}\right)$ in anticyclones (Fig. 3-10i). The exception is during winter, when even though iron concentrations are elevated and local division rates are elevated at any given depth, the redistribution of the biomass across a deeper mixed layer yields suppressed biomass-weighted depth averaged division rates. The opposite is true of cyclones.

\subsection{Discussion}

\subsubsection{Comparison of theoretical, simulated and observed mixed layer depth anomalies}

Theoretically, cyclones and anticyclones should preferentially induce shallower and deeper

mixed layers respectively, however, this is not apparent in the overall distribution of simulated 
eddies. This is largely because small eddies passing through regions with shallow background mixed layers preferentially exhibit "backwards" $M L D^{\prime}$. This may be partially explained by mode-water eddies, which are not distinguished from anticyclones in the tracking algorithm [Faghmous et al. 2015]. Mode-water eddies dome the seasonal pycnocline but depress the main pycnocline, inducing a $+S S H^{\prime}$ anomaly like anticyclones, but stratifying the surface ocean like cyclones [McGillicuddy 2016; McGillicuddy et al. 2007]. This could explain how some mode-water eddies, misidentified as anticyclones, induce negative $M L D^{\prime}$, but does not explain concurrent positive $M L D^{\prime}$ induced in some cyclones. From a numerical perspective, however, it makes sense that smaller eddies with shallower mixed layers are not resolved as well as larger, deeper eddies. The magnitude of $M L D^{\prime}$ in these eddies is on average half the size $(4.5 \mathrm{~m})$ of the model's vertical resolution $(10 \mathrm{~m})$ and the size cutoff for the radius of "small" eddies $(50 \mathrm{~km})$ is only 5 times larger than the model's nominal horizontal resolution $(10 \mathrm{~km})$. Finally, despite inconsistencies in direction, the magnitude of $M L D^{\prime}$ in these less energetic eddies is still quite small, which agrees with observations of low magnitude anomalies in quiescent Southern Ocean eddies [Hausmann et al. 2017] and the theoretical physical underpinning that smaller eddies should not dramatically deform isopycnals.

The most consistently "backwards" eddies are found under heavy ice cover (fractional coverage above .8; Fig. 3-7b). These under-ice eddies are small $(A m p=2.2 \mathrm{~cm})$, subject to weak background mixing $\left(\overline{M L D}_{C l i m}=69 m\right)$ and typically $(76 \%)$ deform the mixed layer in a direction contradictory to theory. Geographically, they coincide with the high latitude regions where the model overestimates the frequency of eddy activity relative to observations (Fig. 3-3b). Together, it is likely that eddies are not well represented underneath sea ice in the simulation.

Larger eddies with deep background mixing do, however, agree with theory [McGillicuddy 2016] and observations [Hausmann et al. 2017]. Peak $M L D^{\prime}$ is simulated and observed [Hausmann et al. 2017] to occur in the late-winter along Southern Ocean current systems and increase with eddy amplitude. The magnitude of simulated anomalies reported in Fig. 3-5 \& Tab. 3.1 is smaller than observed, but this is largely because anomalies here are averaged over the entire lateral profile of eddies rather than just their core where $M L D^{\prime}$ is maximized [Hausmann et al. 2017]. If the simulated eddy cores are isolated (1/2 the eddy radius), then anomaly magnitudes increase; peak anomalies are on average $59 \mathrm{~m}$ in the core of simulated, deep mixing, late-winter anticyclones, compared to $60 \mathrm{~m}$ in observed late-winter anticyclones within energetic Southern Ocean regions (delineated using the standard deviation of $S S H$ ) [Hausmann et al. 2017]). The same subset of simulated cyclones, however, exhibits anomalies of $-63 \mathrm{~m}$, rather than the less extreme $-30 \mathrm{~m}$ found in the observations. It is unclear why the model does not reproduce this asymmetry in the magnitude of $M L D^{\prime}$, but unlikely that the qualitative nature of light and iron modification in cyclones is fundamentally biased. 


\subsubsection{Comparison of theoretical, simulated and observed iron transport}

Historically, observations have often attributed eddy-induced chlorophyll anomalies to eddy pumping during formation [Falkowski et al. 1991; McGillicuddy et al. 1998; Siegel et al. 1999]. More recent work has, however, identified substantial regional variability in its importance [Gaube et al. 2014a,b] and highlighted regions [Gaube and McGillicuddy 2017; Zhang et al. 2018] and case studies [McGillicuddy et al. 2007] where eddy-induced Ekman pumping instead appears to dominate. McGillicuddy et al. [2007], for example, measured in-situ physical and biological properties of an Atlantic mode-water eddy and found upward vertical velocities of $40 \mathrm{~cm} / d$ that coincided with increased productivity at the core of the eddy. The magnitude of simulated vertical velocities associated with eddy-induced Ekman pumping in the Southern Ocean is on average $16 \mathrm{~cm} / \mathrm{d}$, but exceeds $67 \mathrm{~cm} / \mathrm{d}$ in the strongest 5 percent of eddies. This is in line with a globally observed mean of roughly $10 \mathrm{~cm} / \mathrm{d}$ [Gaube et al. 2014a], and upper bound on the order of $100 \mathrm{~cm} / \mathrm{d}$ [Martin and Richards 2001]. Although observed vertical velocities induced by eddy-pumping in the open ocean can also reach $100 \mathrm{~cm} / d$ [Gaube et al. 2014a; Siegel et al. 1999], they quickly subside after formation. Thus it is not surprising that continuous eddy-induced Ekman pumping can dominate anomalous vertical advection, particularly in the Southern Ocean where wind speeds are strong. Finally, it has been observed that nonlinear submesoscale processes on the periphery of an eddy could also induce unpwelling in anticyclones [Brannigan 2016; Mahadevan et al. 2008]. These effects are not resolved in this simulation, however, they are thought to averaged out over the breadth of an eddy [McGillicuddy et al. 2008] and are would not be expected qualitatively change the direction of anomalous iron transport [Mahadevan et al. 2008].

More surprising is the relative contribution of iron from the vertical mixing flux. The amplified role of eddy-induced Ekman pumping relative to mixed layer modification agrees with work by Zhang et al. [2018], who conclude that the vertical supply of nitrate in Gulf Stream eddies is dominated by eddy-induced Ekman pumping rather than changes vertical turbulent diffusion, but contradicts Song et al. [2018] who conclude mixed layer depth anomalies are the dominant driver of iron into/out of eddies. Song et al. [2018] critically come to this conclusion using an identical model integration to ours but computed the iron budget exclusively over the bulk mixed layer, not considering the distribution of the biomass profile. This is an important distinction in the spring and summer because biomass can exist well below the mixed layer where it continues to be fed by eddy-induced Ekman pumping (Fig. 3-10a, b) but is left out of the [Song et al. 2018] budget. Even in the winter, the strongest anomalous vertical mixing flux of iron is at the base of the mixed layer (Fig. 3-10c, d) while the strongest biomass anomaly is at the surface (Fig. 3-10i, j). Further, a third of the Song et al. [2018] data is not actually within a coherent eddy structure (see $\mathbf{3 . 2 . 5}$ ), and not likely to experience the 
systematic eddy-induced Ekman pumping associated with rotating vortices.

Nevertheless, our results should not diminish importance of the mixed layer modification. For instance, wintertime eddies with deep mixed layer anomalies $\left(M L D^{\prime}>+10 m\right)$, exhibit biomass-weighted depth-averaged iron anomalies 350\% larger on average than those in eddies with shallow mixed layer anomalies $\left(M L D^{\prime}<-10 m\right)$. Instead, we want to caution that the anomalous iron supply from mixed layer modification is limited seasonally to the winter and regionally to deep mixing environments like the ACC. Eddy-induced Ekman pumping, however, appears to modify the iron supply with much more seasonal and regional consistency. Finally, it is important to remember that both mechanisms modify the vertical iron profile and are thus not independent. For instance, even once the mixed layer shoals, eddy-induced Ekman pumping in anticyclones can continue to advect iron that was previously mixed higher up the water column, which is consistent with the hypothesis proposed by Song et al. [2018] that winter preconditioning leaves some remnant of unused iron limitation available to the summer anticyclones. Eddy-induce Ekman pumping provides a mechanistic explanation of how this iron can be accessed even if it remains below a shallow mixed layer.

We focus on vertical nutrient pathways because these can supply/suppress new nutrients capable of stimulating/stifling new production, but caution that estimates of lateral transport mechanisms operate at a similar magnitude. Lateral and vertical processes are not entirely independent, but because the estimated supply of anomalous iron from horizontal mechanisms is similar in magnitude to that from vertical ones (Fig. 3-9), it could be reasonably assumed that about half of the simulated iron anomaly in eddies represents the accumulation of new iron from enhanced or suppressed vertical transport from depth.

\subsubsection{Comparison of theoretical, simulated and observed anomalous divi- sion rates}

We can confirm that simulated Southern Ocean eddies explicitly modify the division rates of the phytoplankton populations within them as inferred by past studies from chlorophyll anomalies (e.g. Frenger et al. [2018]), correlative relationships (e.g. Gaube et al. [2014a]), and simulated shifts in light and iron availability (e.g. Song et al. [2018]). Our results clearly demonstrate a consistent, population-averaged response; divisions rates are, on average, elevated in anticyclones and depressed in cyclones. This is implicitly consistent with observations [Frenger et al. 2018] and simulations [Song et al. 2018] of elevated and depressed [Chl $]_{S}$ inside of anticyclones and cyclones respectively. Further investigation into the simultaneous effects of dilution [Behrenfeld 2010], grazing [Quéré et al. 2016], and lateral advection [Frenger et al. 2018], however, is warranted to confirm that variability in depth-averaged division rates is the dominant driver of variability in surface chlorophyll concentrations. 
Moreover, our results systematically demonstrate how $\mu_{\Sigma}^{\prime}$ can deviate from the mean, helping corroborate the hypothesis that different mechanisms can dominate the biogeochemical response to eddy perturbation [Gaube et al. 2014b]. Specifically, observations [Frenger et al. 2018] and simulations [Song et al. 2018] have identified a seasonal flip in the direction of $[C h l]_{S}^{\prime}$ along the ACC. Frenger et al. [2018] find that this reversal can not be explained by quantitative estimates of lateral stirring or trapping and infer that variability $[C h l]_{S}^{\prime}$ must be driven by rate-based mechanisms. Song et al. [2018] go on to show that this seasonal variability is replicated in this simulation and associated with a corresponding shift in the dominance of light vs. iron limitation. Here we go a step further and confirm that this shift is, in fact, manifest in $\mu_{\Sigma}^{\prime}$ and is mechanistically consistent increasing light limitation observed during deep mixing events in the Southern Ocean [Boyd 2002; Fauchereau et al. 2011; Moore and Abbott 2002; Venables and Meredith 2009].

\subsubsection{Basin scale iron budget}

Eddy induced nutrient fluxes have been proposed as a mechanism to help close global nutrient budgets [Doddridge and Marshall 2018; McGillicuddy et al. 2003]. Here, eddies are shown to induce anomalous iron transport on the order of $5-20 \%$ of background climatologies. However, due to the symmetry between cyclones and anticyclones much of this effect is cancelled out across larger geographical areas, prompting the question of whether or not there is a rectified effect at the basin scale after averaging across eddies of both polarities.

Averaged across the entire Southern Ocean the gridcell of the average phytoplankton inside simulated eddies is supplied with an extra $3.2 \mathrm{nmol} / \mathrm{m}^{3}$ of iron per year via anomalous vertical advection. This value was computed by weighting the anomalous iron supply rate $\left({\frac{d[F e]^{\prime}}{d t}}_{W, \Sigma}\right)$ by eddy size and biomass before averaging across all eddy realizations in the Southern Ocean. It is possible for the anticyclonic signal to dominate, despite symmetric modifications to the eddy-induced Ekman pumping velocity, because of biological uptake and the shape of the iron profile which increases with depth. In the subtropical gyres, for instance, where the mean state is one of downwelling, this means that additional downwelling in cyclones can not drain much extra iron out of iron deplete surface waters, whereas anomalous upwelling in anticyclones can provide substantial iron from iron rich deep waters, enabling a net supply of iron across eddies at the basin scale. Unlike the subtropical gyres though, the mean vertical velocity in the Southern Ocean is upwards (see Fig. B-4; Morrison et al. [2014] and Tamsitt et al. [2017]). This means that anomalous eddy induced downwelling is not just pumping iron out of the surface but, more importantly, preventing iron at depth from being upwelled. This is why there is a consistent anomalously downward advection flux of iron in cyclones (see Fig. 3-8e,

g) even if there is very little iron at the surface. In turn, it is more likely that the anomalous 
supply of iron from vertical advection will be symmetric across cyclones and anticyclones and more apt to average out in the Southern Ocean. Thus, the rectified effect should be expected to be smaller in the Southern Ocean than in the subtropical basins where cyclones are less capable of suppressing the vertical iron supply relative to the background state. See Table 3.2a for a seasonal breakdown of the basin scale anomalous iron supply rate from vertical advection in Southern Ocean eddies.

The spatially averaged anomalous supply of iron via mixing $\left(\frac{d[\mathrm{Fe}]^{\prime}}{d t} \mathrm{Mix, \Sigma}\right)$ is $7.3 \mathrm{nmol} / \mathrm{m}^{3}$ of iron per year. Even though the average anomalous supply rate in individual eddies is almost an order of magnitude smaller for mixing than advection, the rectified effect is actually twice as large. Despite roughly symmetric mixed layer modification in cyclones and anticyclones this is possible, again, because of the shape of the iron profile. Relative to surrounding waters, a deeper mixing anticyclone will entrain more iron than a shallower mixing cyclone fails to entrain because the iron concentration increases with depth. Regionally though, this effect is actually reversed in the Patagonian basin where iron availability decreases with depth due to heavy aeolian deposition (see Fig B-3). See Table 3.2b for a seasonal breakdown of the basin scale anomalous iron supply rate from vertical mixing.

Together, the average anomalous eddy-induced vertical iron supply rate is $10.5 \mathrm{nmol} / \mathrm{m}^{3}$ of iron per year throughout the Southern Ocean. This increases to $14 \mathrm{nmol} / \mathrm{m}^{3}$ of iron per year when only considering large eddies. These values are on the order of roughly $1 \%$ of background rates and $10 \%$ of local effects in eddies of a particular polarity. It is not surprising net effect of eddies on the iron supply is small given the symmetric behavior in cyclones and anticyclones and the state of background upwelling in the Southern Ocean that allows cyclones to stifle the vertical transport of iron. Still, there appears to be a pathway for simulated Southern Ocean eddies to induce a rectified iron supply at the basin scale. Including non-linear Ekman pumping, which is not resolved in this simulation, would likely increase the strength of this supply. Non-linear Ekman pumping induced by the vorticity gradient should be expected to induce overturning cells along the edge of eddies [Mahadevan et al. 2008]. This would open another pathway for net vertical iron supply so long as the timescale of overturning is slow enough for phytoplankton to utilize upwelled iron before water is subsequently subducted back out of the euphotic zone. While there is, in fact, evidence for a rectified iron supply in the Southern Ocean it is important to recognize that even though it is probably underestimated in this simulation it likely remains much smaller than in the subtropical gyres as it is apt to average out to a larger degree over the basin scale. 


\subsection{Conclusions}

Our findings demonstrate the comprehensive manner by which simulated eddies modify the phytoplankton populations within them from the bottom-up. Throughout the Southern Ocean, simulated cyclones and anticyclones induce a largely symmetric physical and biogeochemical response. Eddy-induced Ekman pumping, driven by strong Southern Ocean Winds, consistently stimulates the upward advection of iron and improves population-averaged iron availability across all anticyclones. Deep mixed layer depth anomalies are found in large anticyclones with deep background mixing, such as those in the wintertime ACC, and provide additional iron to phytoplankton via an increased upward mixing flux. This extra iron, however, comes at the price of exacerbated light stress, stymieing the ability of iron to improve population averaged division rates. The opposite is true in cyclones. The net effect is that population-averaged division rates are widely elevated in anticyclones and depressed in cyclones, but the magnitude of these anomalies is closely tied to the depth of the background mixed layer. If the background mixed layer is deep enough, anticyclones, for instance, can exhibit depressed division rates despite substantially improved iron availability. This is most evident in the deep winter mixing Pacific sector of the ACC.

\subsection{Acknowledgments}

The CESM project is supported by the National Science Foundation and the Office of Science (BER) of the U.S. Department of Energy. Computing resources were provided by the Climate Simulation Laboratory at NCAR's Computational and Information Systems Laboratory (CISL), sponsored by the National Science Foundation and other agencies. This research was enabled by CISL compute and storage resources. TR and SCD acknowledge support from the National Aeronautics and Space Administration Ocean Biology and Biogeochemistry Program (NNX14AL86G) and the National Science Foundation Polar Programs award 1440435 (Antarctic Integrated System Science) to the Palmer LTER program. Please contact trohr@mit.edu for further questions or to access to data. 


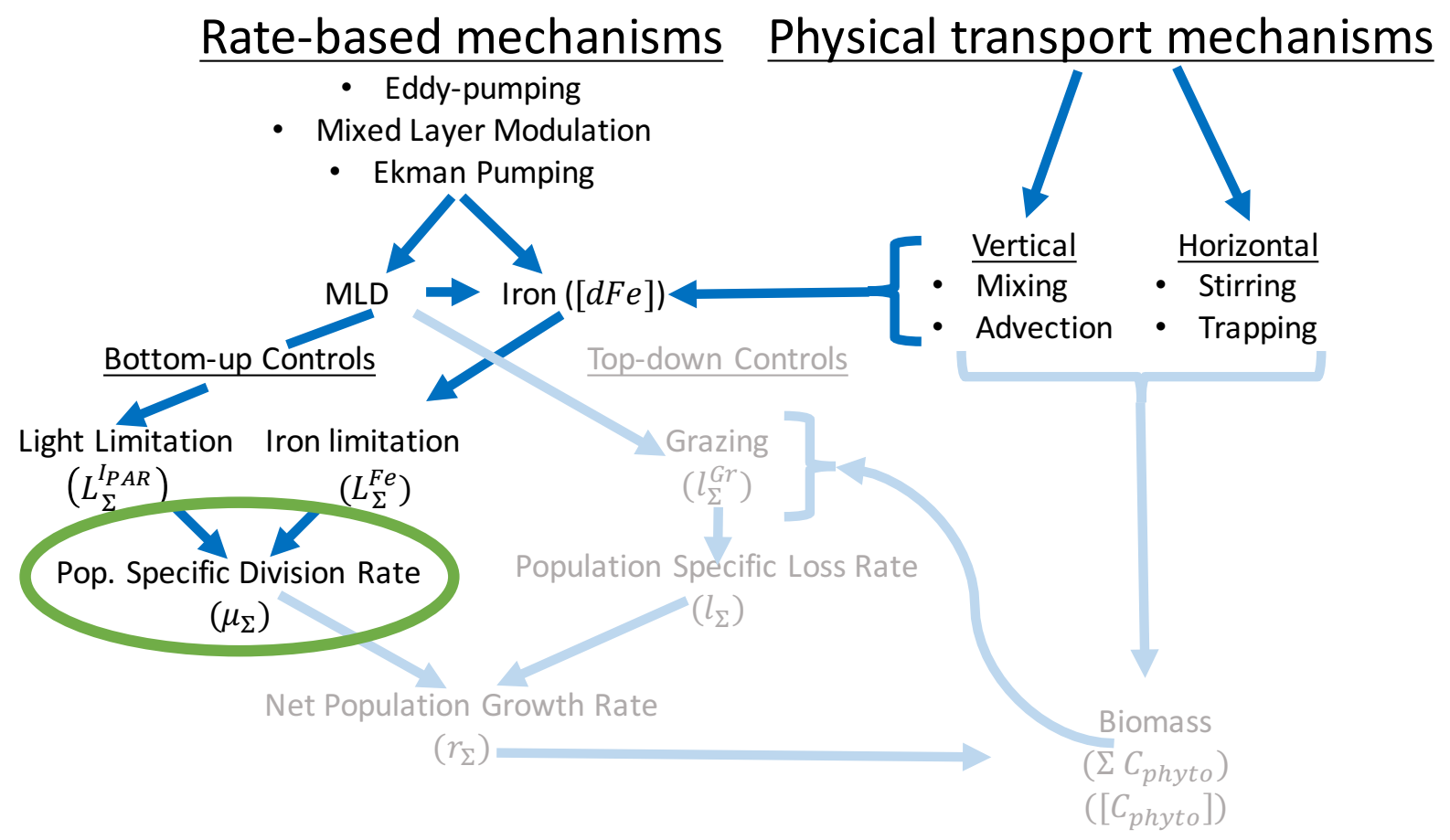

Figure 3-1: Pathways for eddy influence on biomass. Idealized flow chart of how physically induced processes in eddies can influence biogeochemistry, and ultimately biomass. Highlighted processes, pathways and tracers are examined here. All remaining pathways (faded) leading to the ultimate simulated biomass anomaly are considered in Chapter 4 . 


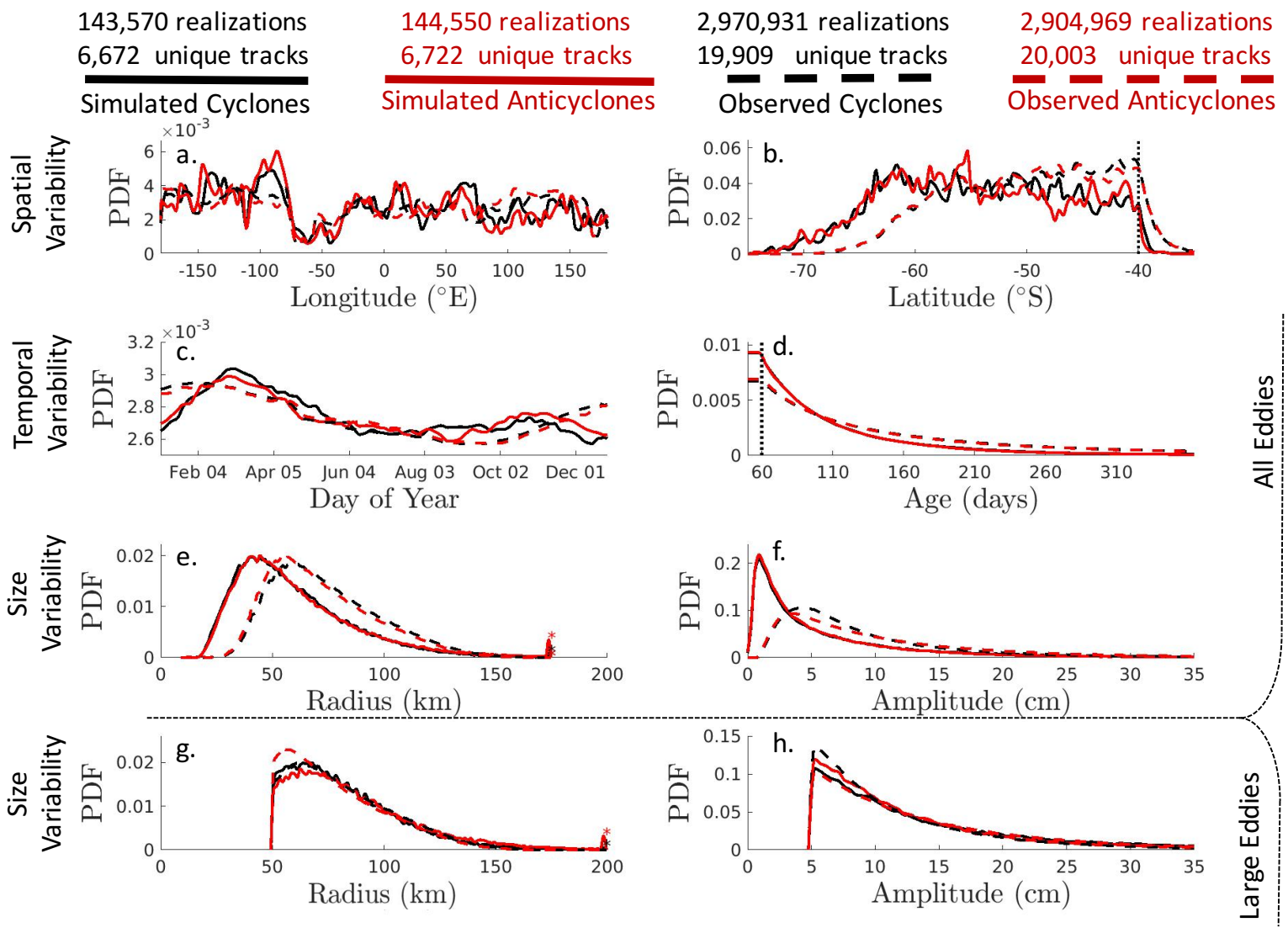

Figure 3-2: Simulated and observed eddy demographics. The probability distribution of all eddy realizations is plotted for various attributes of simulated cyclones (solid black) and anticyclones (solid red) alongside observed cyclones (dashed black) and anticyclones (dashed red). The entire set of Southern Ocean eddy realizations (see 3.2.6) is included in a-f. Vertical dashed lines indicate the constraints that eddy tracks must cross south of $40^{\circ} S$ (b) and last at least 60 days (d). Note that eddies shorter than 60 days were removed from the observational tracks for a consistent comparison. Variability in the size of strictly large eddy realizations $\left(L_{S}>50 \mathrm{~km} ;\right.$ Amplitude $\left.>5 \mathrm{~cm}\right)$, which comprise $35 \%$ of simulated realizations and $60 \%$ of observed realizations, are plotted in $\mathbf{g}-\mathbf{h}$. 

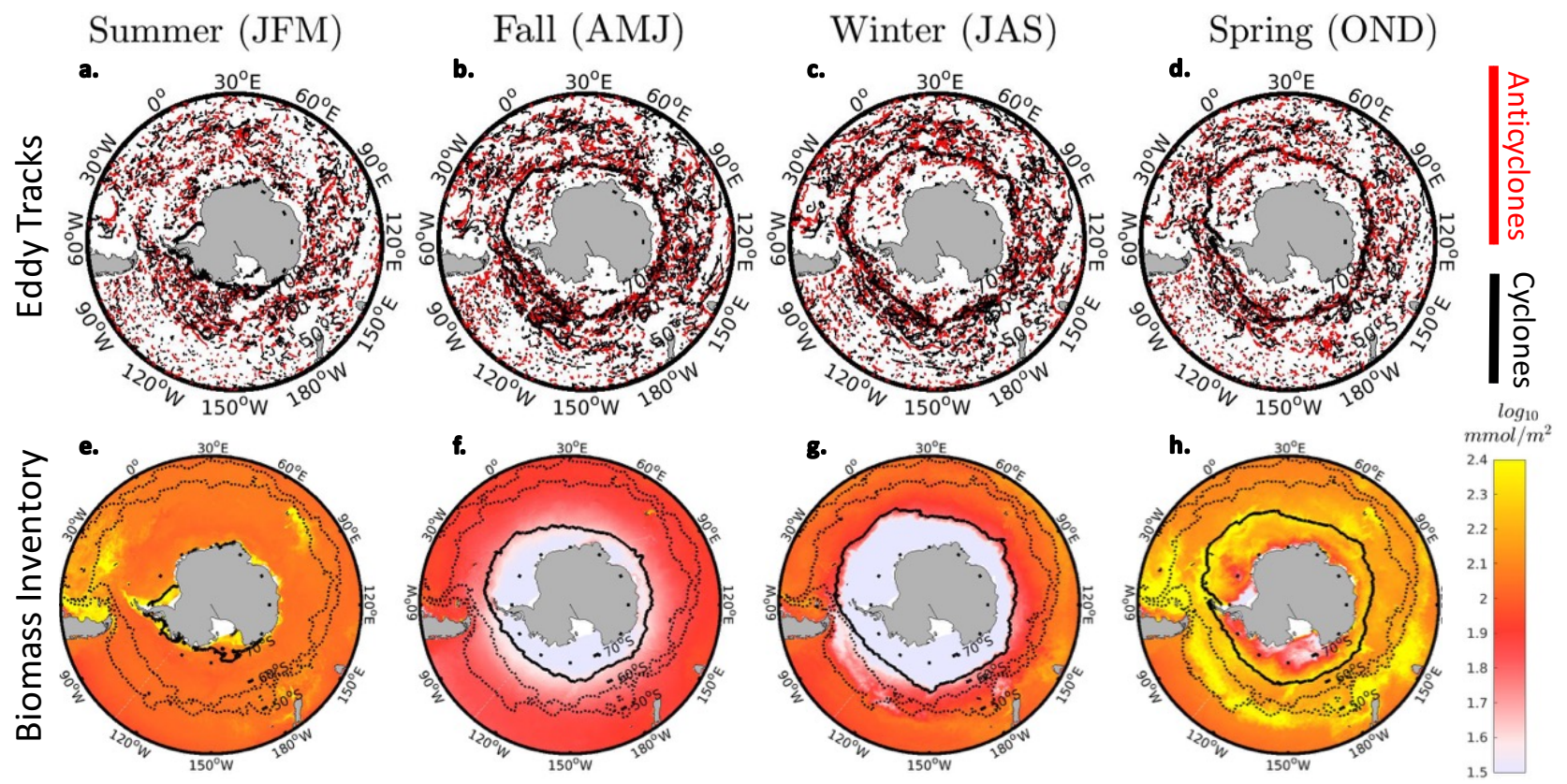

Figure 3-3: Seasonal distribution of eddy tracks and biomass inventory. (a-d) The seasonal distribution of eddy tracks is plotted for cyclones (black) and anticyclones (red). Tracks are seasonally delineated by the season of their median day. (e-h) The corresponding seasonal depth-integrated biomass climatology is plotted in log space. The seasonal mean $10 \%$ ice contour is plotted in each subplot with a solid black line and the ACC (see 3.2.6) is plotted with a dashed black line on plots e-h 

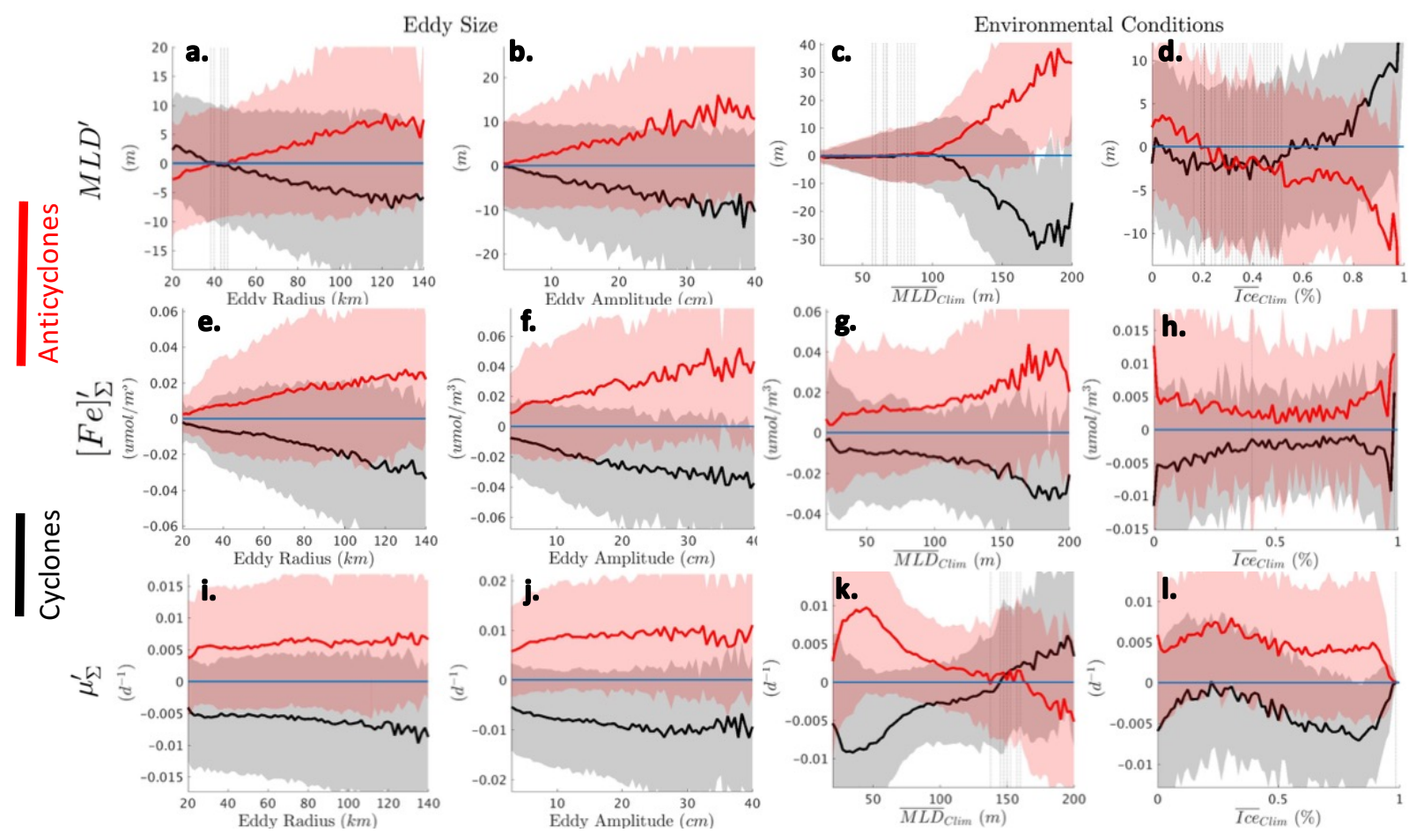

Figure 3-4: Variability in eddy anomalies. Variability in $(\mathbf{a}, \mathbf{b}, \mathbf{c}, \mathbf{d}) M L D^{\prime},(\mathbf{e}, \mathbf{f}, \mathbf{g}, \mathbf{h})[F e]_{\Sigma}^{\prime}$, and $(\mathbf{i}, \mathbf{j}, \mathbf{k}, \mathbf{l}) \mu_{\Sigma}^{\prime}$ as a function of eddy radius (first column), eddy amplitude (second column), the climatologic mixed layer depth (third column) and the climatologic ice fraction (forth column). Cyclones are plotted in black. Anticyclones are plotted in red. Error bars (shaded) denotes $+/-1$ standard deviation. Bins that are statistically insignificant from 0 at the $95 \%$ confidence level are hatched out with vertical dotted lines. 

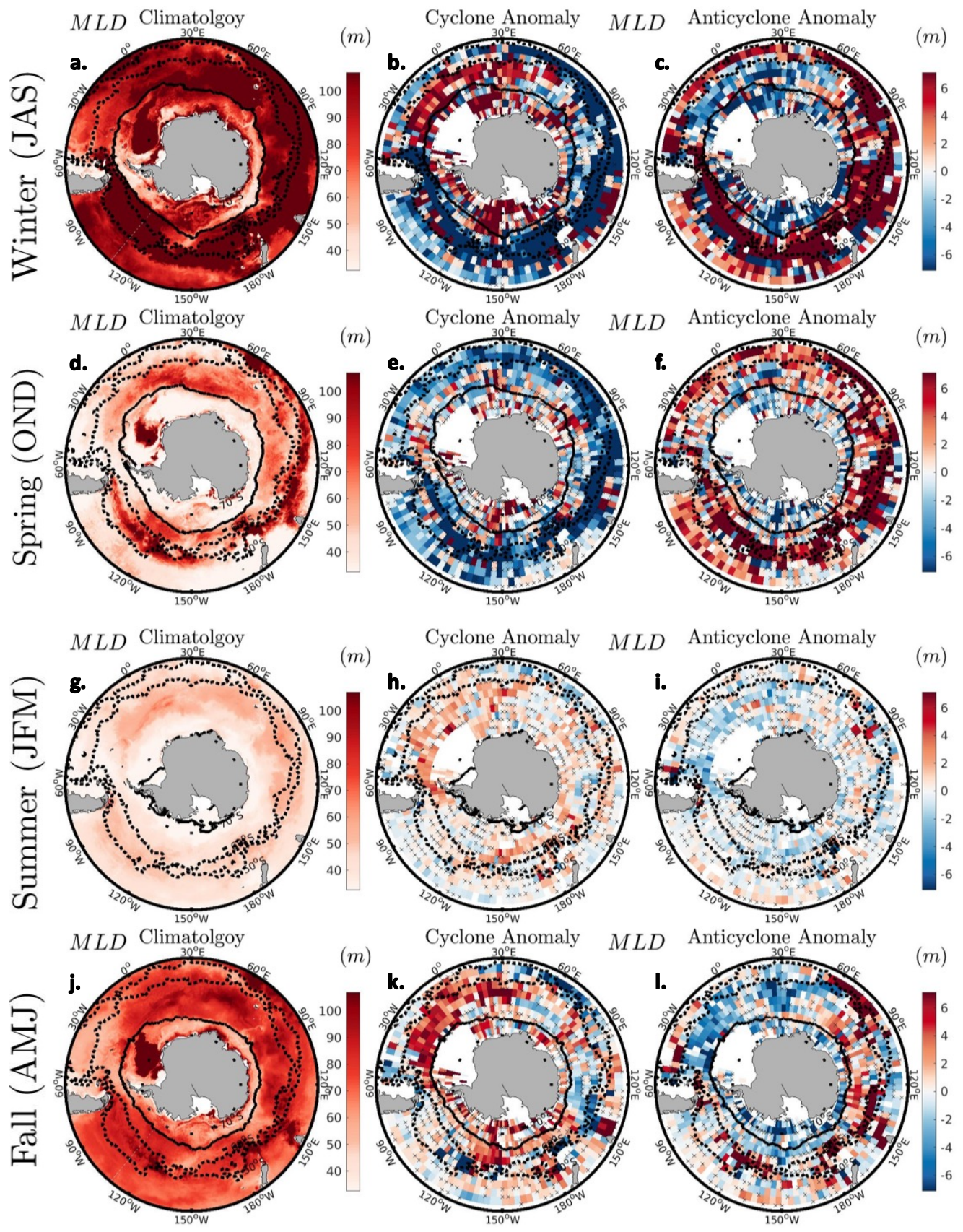

Figure 3-5: Seasonal $M L D$ climatology and eddy anomalies. $(\mathbf{a}, \mathbf{d}, \mathbf{g}, \mathbf{j})$ The simulated climatologic $M L D$. $(\mathbf{b}, \mathbf{e}, \mathbf{h}, \mathbf{k})$ The simulated mean $M L D^{\prime}$ in all cyclones. $(\mathbf{c}, \mathbf{f}, \mathbf{i}, \mathbf{l})$ The simulated mean $M L D^{\prime}$ in all anticyclones. Data is seasonally binned as denoted in each row. Eddy realizations are further averaged over $3^{\circ} \times 3^{\circ}$ spatial bins. Each bin may contain multiple realizations from the same eddy track provided that each time step falls within the seasonal threshold. Bins that are not statistically different from 0 at the $95 \%$ confidence level are denoted with an ' $x$ '. The $10 \%$ ice contour is denoted in black. The ACC $\left(-80 \mathrm{~cm}<\overline{S S H}_{C l i m}<-20 \mathrm{~cm}\right)$ is denoted in with a dashed black line. 

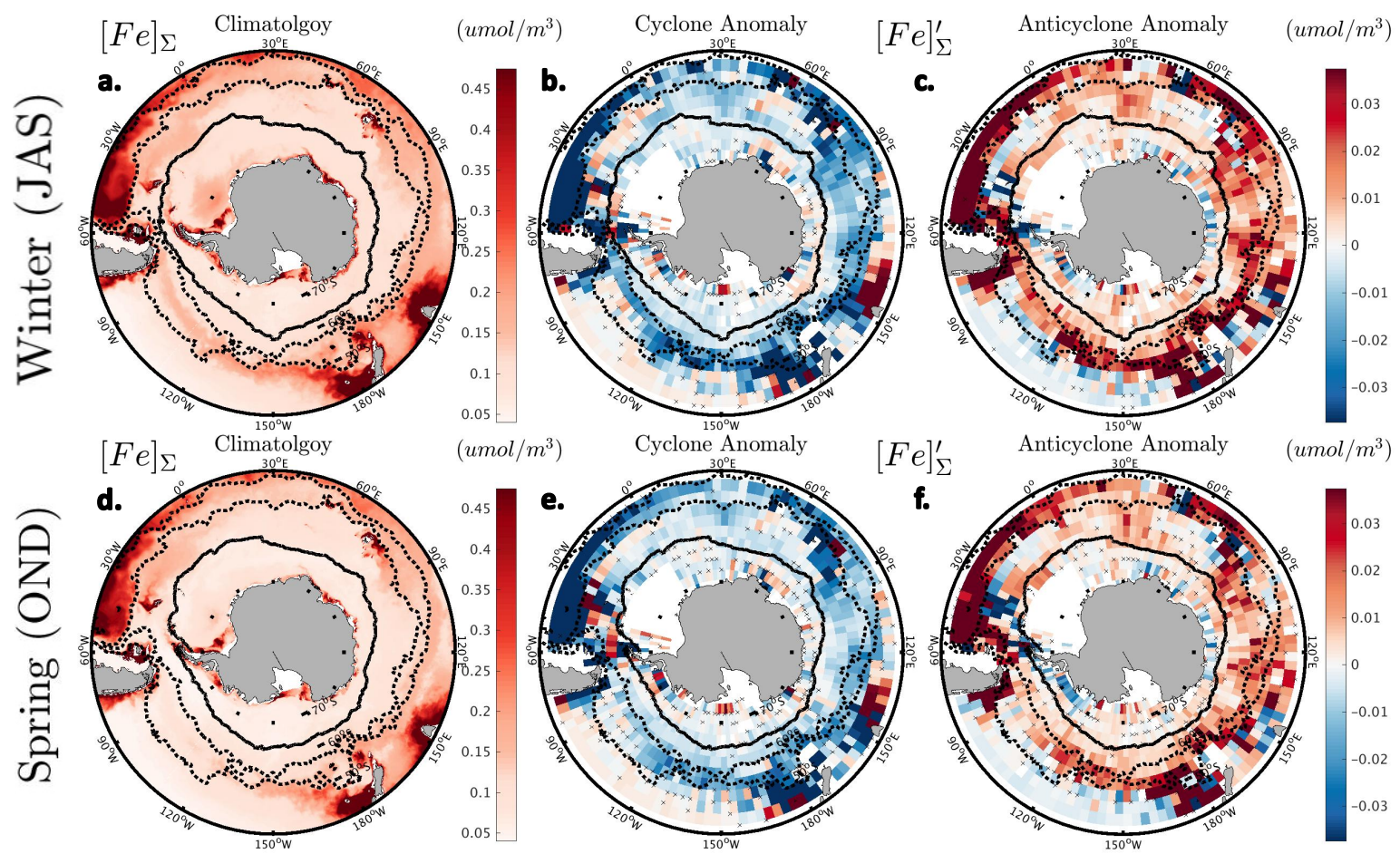

$\left(\mathrm{umol} / \mathrm{m}^{3}\right)$
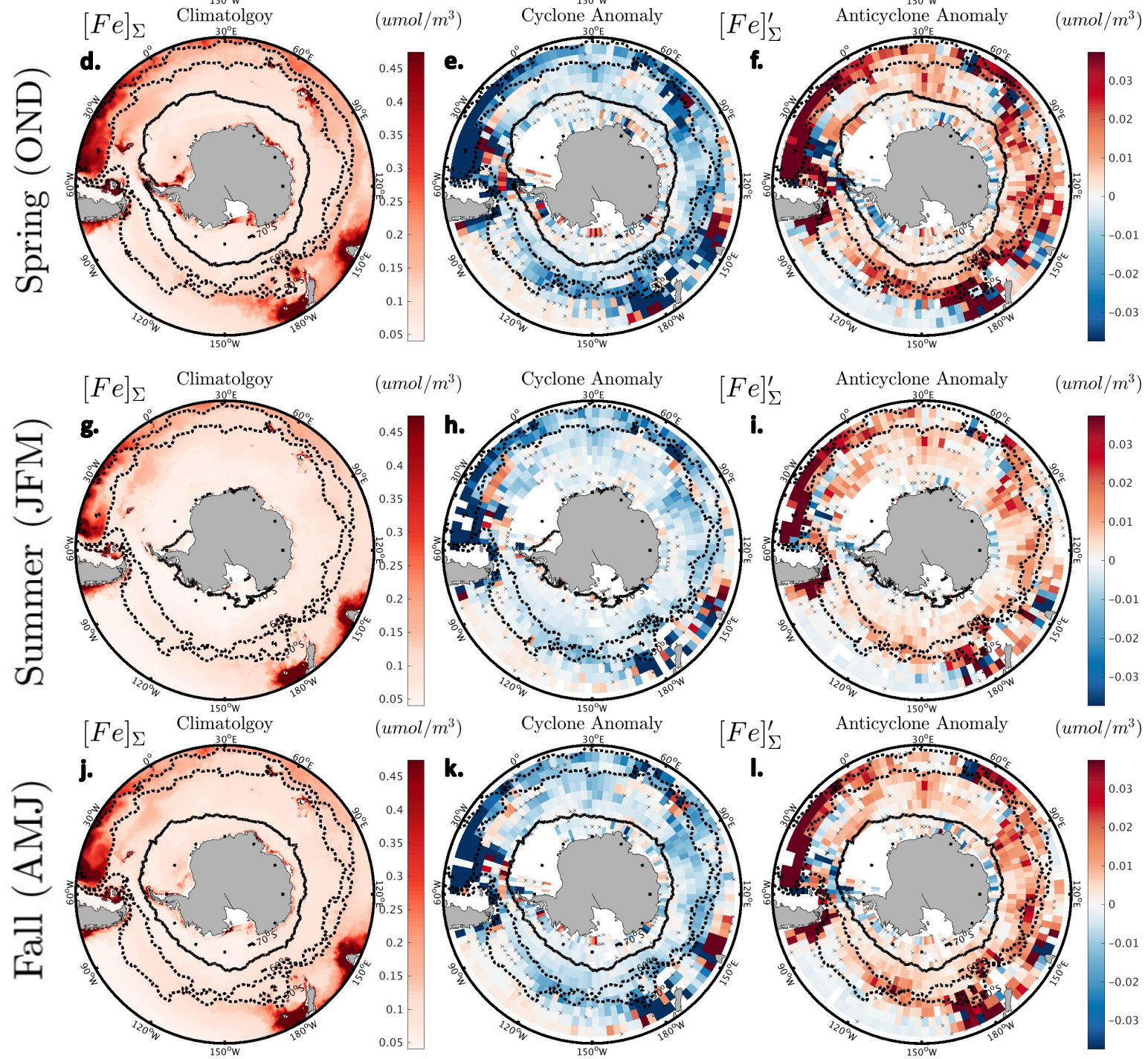

Figure 3-6: Seasonal $[\mathrm{Fe}]_{\Sigma}$ climatology and eddy anomalies. Same as Fig. 3-5 except for $[F e]_{\Sigma}$. 

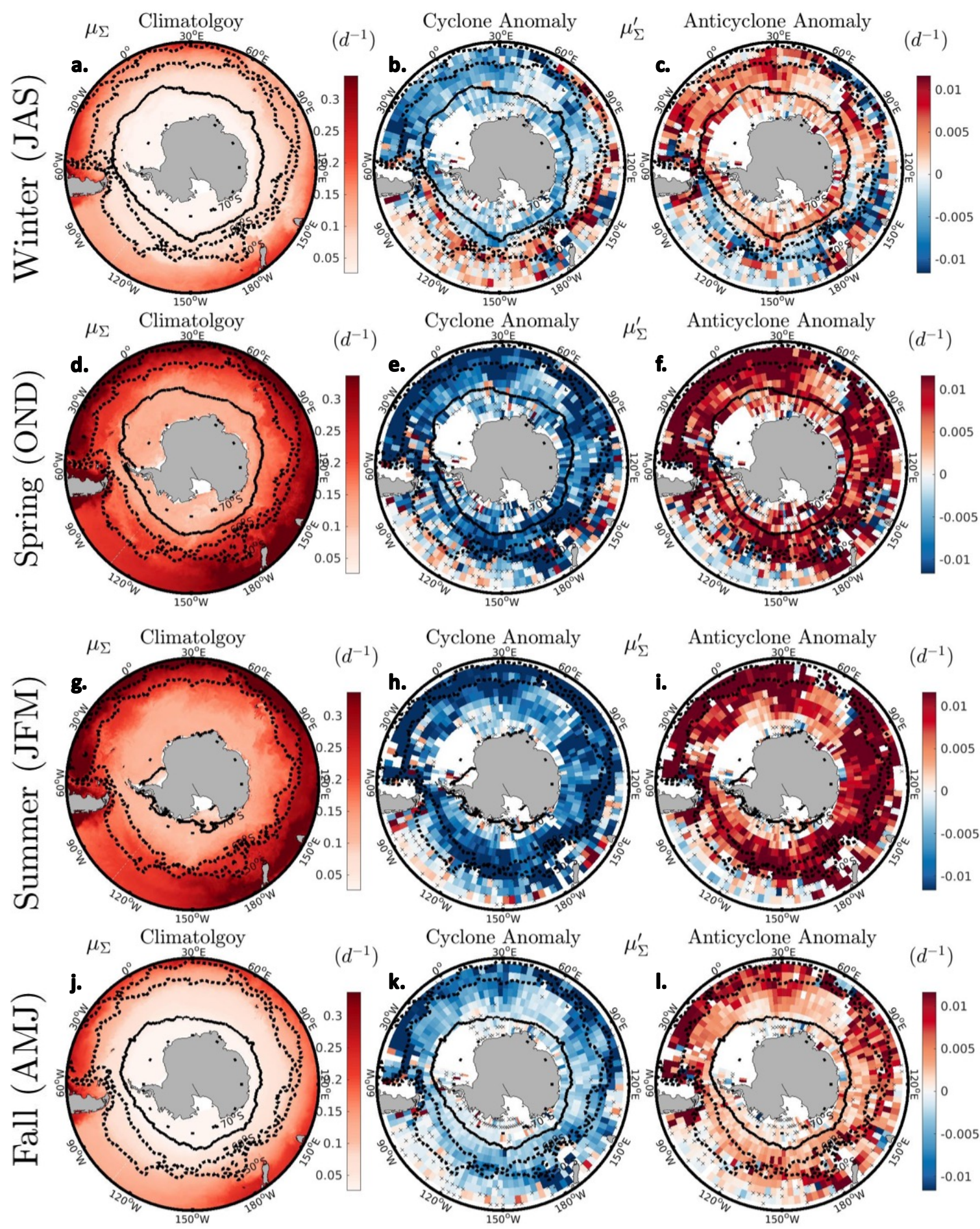

Figure 3-7: Seasonal $\mu_{\Sigma}$ climatology and eddy anomalies. Same as Fig. 3-5 except for $\mu_{\Sigma}$. 


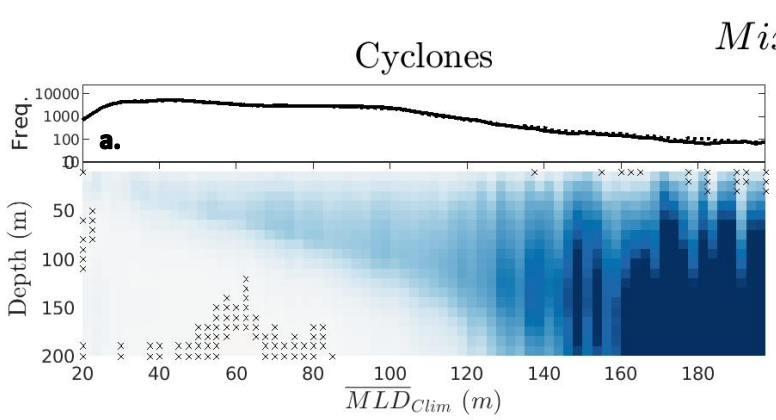

$\operatorname{Mix} x_{F e}^{\prime}\left(\frac{\mu m o l}{m^{2} s}\right)$

\section{Anticyclones}
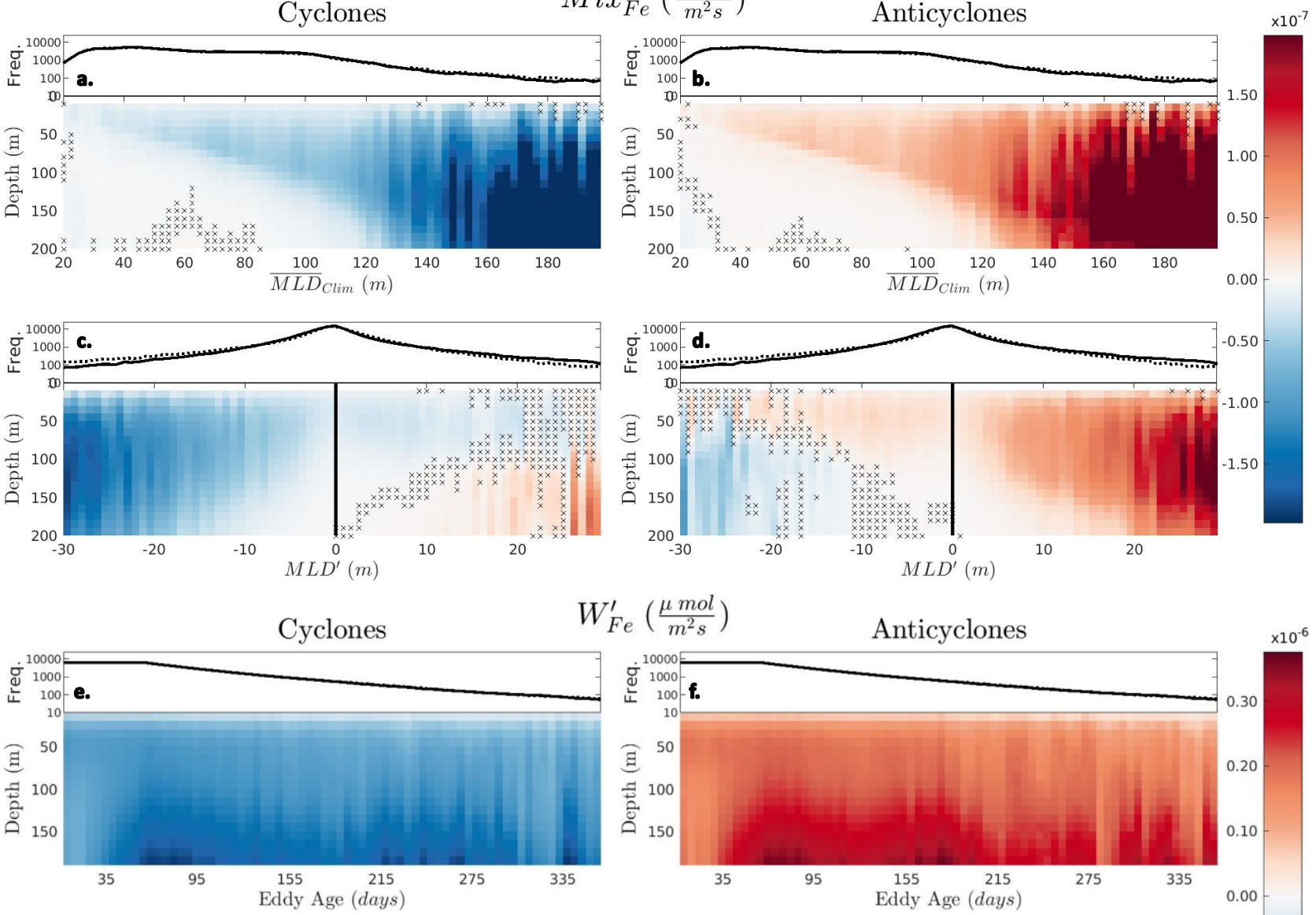

$W_{F e}^{\prime}\left(\frac{\mu m o l}{m^{2} s}\right)$
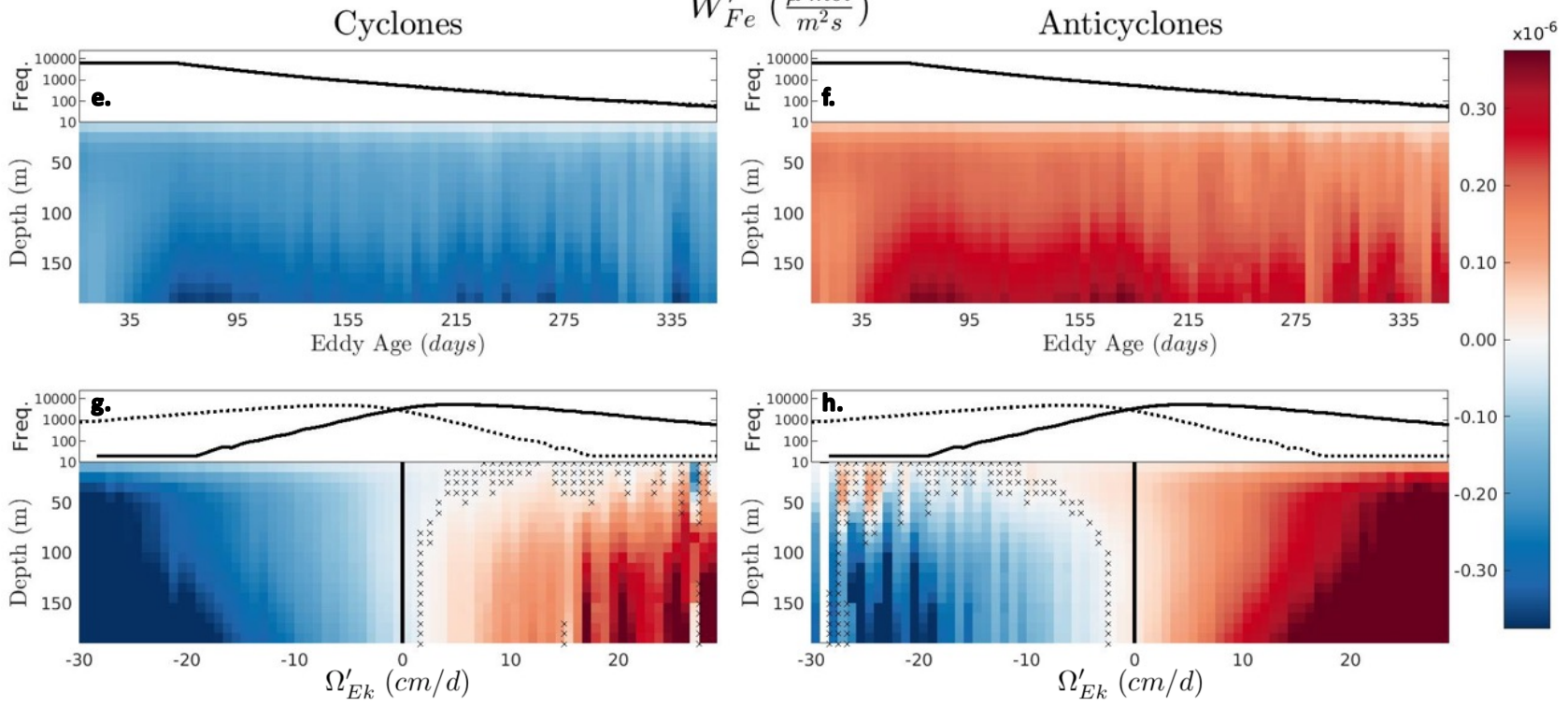

Figure 3-8: Vertical iron transport profiles. (a, b, c, d) Depth profiles of the anomalous vertical mixing flux of iron $\left(M i x_{F e}^{\prime}\right)$ and $(\mathbf{e}, \mathbf{f}, \mathbf{g}, \mathbf{h})$ the anomalous vertical advection flux of iron $\left(W_{F e}^{\prime}\right)$ averaged across all cyclones (left) and anticyclones (right). $M i x_{F e}^{\prime}$ is plotted as a function of (a, b) the background climatologic mixing depth $\left(\overline{M L D}_{\text {Clim }}\right)$ and $(\mathbf{c}, \mathbf{d})$ the mixed layer depth anomaly $\left(M L D^{\prime}\right) . W_{F e}^{\prime}$ is plotted as a function of $(\mathbf{e}, \mathbf{f})$ the eddy age and $(\mathbf{c}, \mathbf{d})$ the anomalous Ekman velocity $\left(\Omega_{E k}^{\prime}\right)$. Bins with anomalies that are statistically insignificant from 0 at the $95 \%$ confidence level are marked with an ' $x$ '. The frequency of eddy realizations that fall on each bin on the $\mathrm{x}$-axis is plotted above the corresponding profiles. Cyclonic (solid lines) and anticyclonic (dashed lines) distributions are included in each plot for ease of comparison. 
Time integrated anomalous iron supply from

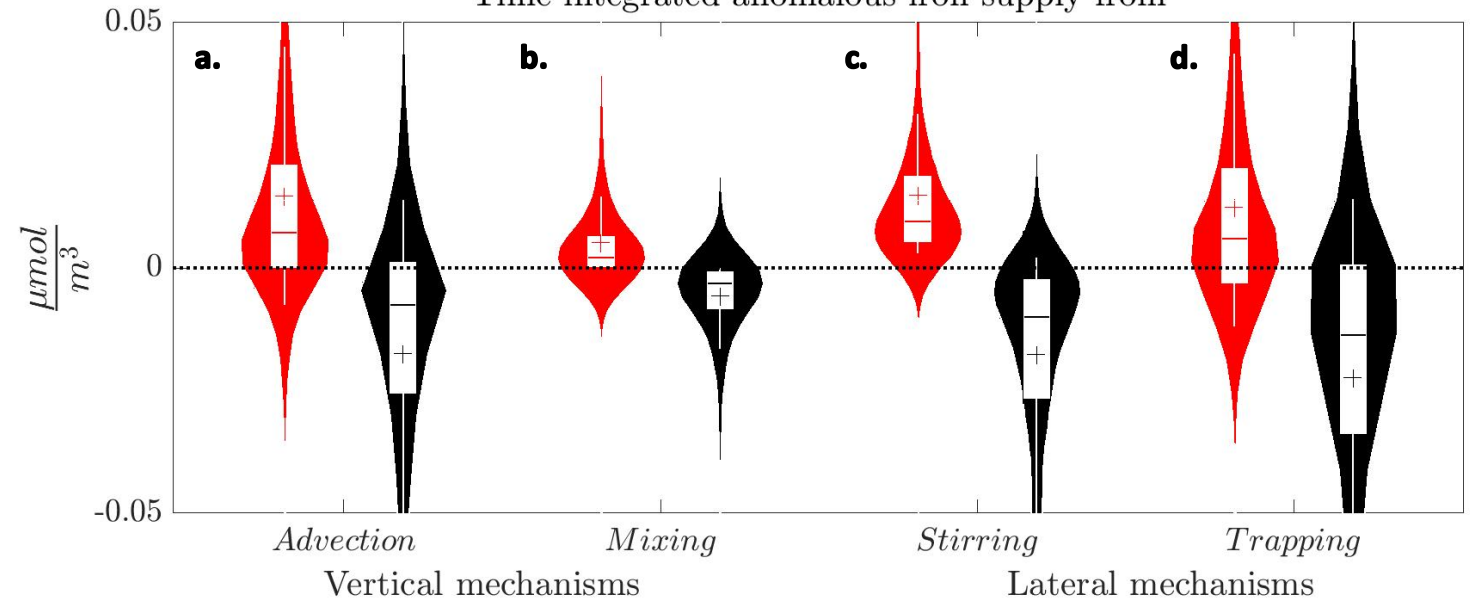

Figure 3-9: Sources of anomalous iron. Violin plots provide the distribution of the total anomalous iron supplied supplied to cyclones (black) and anticyclones (red) via vertical and horizontal mechanisms. Only large eddies within the ACC are considered (see Sec. 2.6). (a, b) Vertical transport mechanisms, Advection and Mixing quantify the actual anomalous time integrated, population averaged supply of iron into eddies over their lifetime (i.e. $\left.\int_{\text {formation }}^{\text {realization }} \frac{d[\mathrm{Fe}]^{\prime}}{d t}{ }_{M i x, \Sigma} d t\right)$. (c, d) Horizontal transport mechanisms, Stirring and Trapping, represent an estimate for the magnitude and direction of anomalies produced solely by lateral processes and are based on climatologic gradients, rather than the actualized anomaly fields. Boxes are defined by the $25^{t h}$ and $75^{t h}$ percentile. Whiskers (white line) extend to $10^{t h}$ and $90^{t h}$ percentile. Medians are denoted with a horizontal line and means are denoted with a + symbol. 

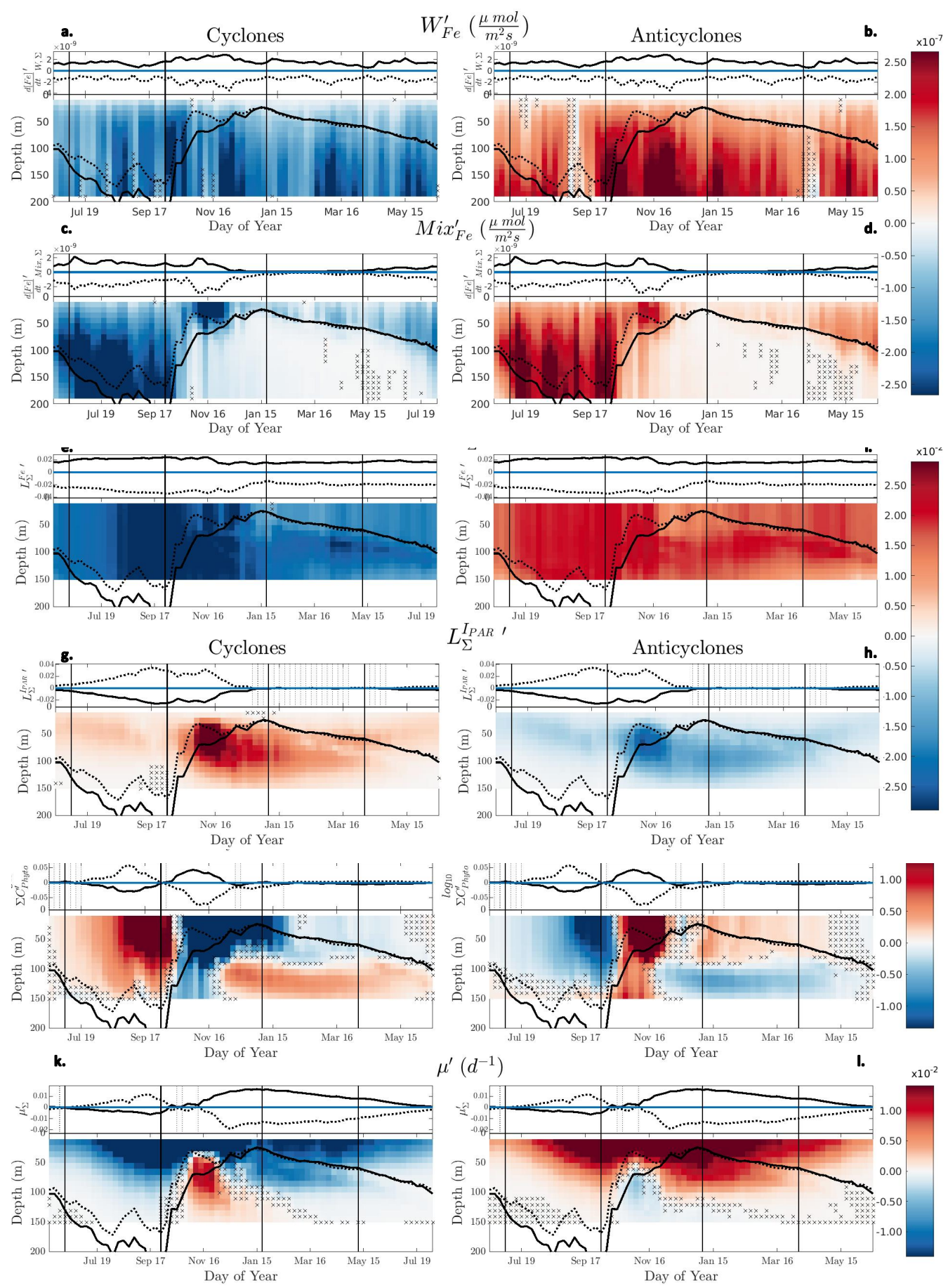

Figure 3-10: Seasonal variability of anomalies in large eddies found with in the deep mixing Pacific sector of the ACC (see 3.2.6). Qualifying eddy realizations that occur on the same day are binned together and averaged. In all, 9087 realizations from 596 unique tracks are included. Profiles of seasonal variability in $L^{I_{P A R^{\prime}}}, L^{F e \prime}, W_{F}^{\prime} e, M i x_{F}^{\prime} e,\left[C_{\text {phyto }}\right]^{\prime}$, and $\mu^{\prime}$ are plotted for cyclones on the left, and anticyclone on the right. The mean $M L D$ in cyclones (dashed) and anticyclones (solid) is overlaid. Bins with anomalies that are statistically insignificant from 0 at the $95 \%$ confidence level are marked with an ' $x$ '. Above each profile plot is a plot of the corresponding depth-averaged population mean. Bins in which the difference between cyclone and anticyclone anomalies are statistically insignificant at the $95 \%$ confidence level are marked with a dash vertical line. Solid vertical lines delineate seasons. 
Cyclones

\begin{tabular}{|c|c|c|c|}
\hline & Large \& Deep & All & Small \& Shallow \\
\hline Realizations & $9364(6.5 \%)$ & $143570(100 \%)$ & $44507(31 \%)$ \\
\hline Unique Tracks & $1188(18 \%)$ & $6672(100 \%)$ & $5238(79 \%)$ \\
\hline
\end{tabular}

Statistical distribution of eddy anomalies

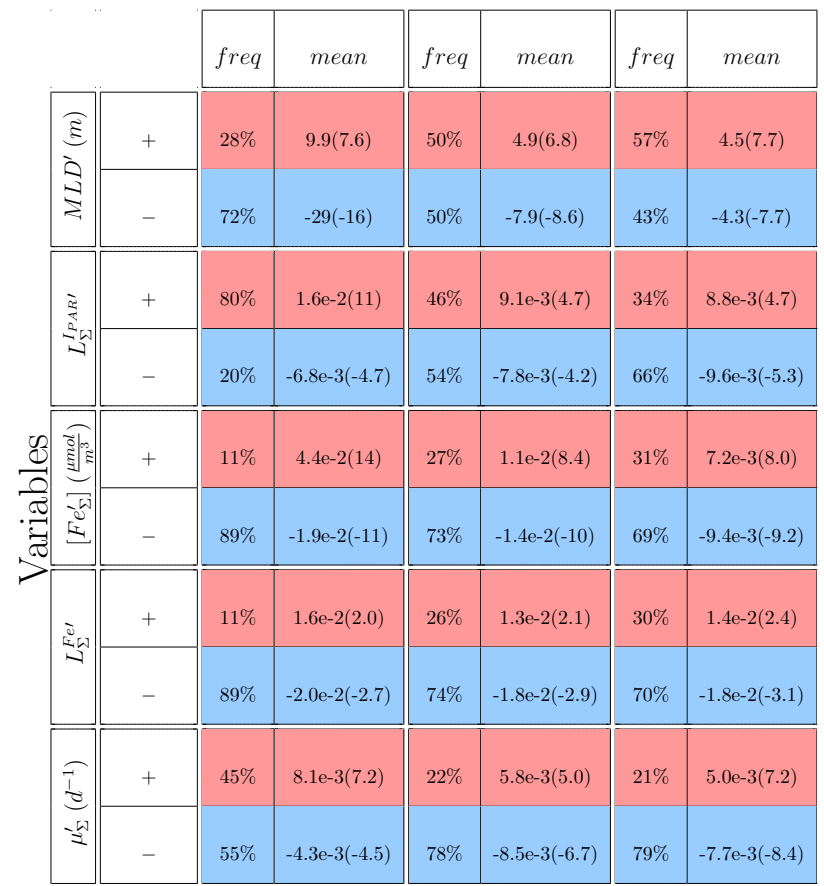

Antiyclones

\begin{tabular}{|c||c||c|}
\hline Large \& Deep & All & Small \& Shallow \\
\hline $9910(6.9 \%)$ & $144550(100 \%)$ & $58086(40 \%)$ \\
\hline $1179(17 \%)$ & $6742(100 \%)$ & $5282(78 \%)$ \\
\hline
\end{tabular}

\begin{tabular}{|c|c|c|c|c|c|}
\hline freq & mean & freq & mean & freq & mean \\
\hline $78 \%$ & $27(18)$ & $51 \%$ & $7.8(9.0)$ & $42 \%$ & $4.1(7.5)$ \\
\hline $22 \%$ & $-7.2(-5.6)$ & $49 \%$ & $-4.7(-6.8)$ & $58 \%$ & $-5.1(-8.3)$ \\
\hline $14 \%$ & $5.2 \mathrm{e}-3(3.6)$ & $51 \%$ & $7.9 \mathrm{e}-3(4.2)$ & $64 \%$ & $9.9 \mathrm{e}-3(5.8)$ \\
\hline $86 \%$ & $-1.6 \mathrm{e}-2(-10)$ & $49 \%$ & $-8.8 \mathrm{e}-3(-4.6)$ & $36 \%$ & $-7.7 \mathrm{e}-3(-4.0)$ \\
\hline $91 \%$ & $2.1 \mathrm{e}-2(14)$ & $71 \%$ & $1.4 \mathrm{e}-2(12)$ & $66 \%$ & $9.9 \mathrm{e}-3(11)$ \\
\hline $9 \%$ & $-3.4 \mathrm{e}-2(-5.6)$ & $28 \%$ & $-1.1 \mathrm{e}-2(-7.7)$ & $34 \%$ & $-7.9 \mathrm{e}-3(-8.2)$ \\
\hline $92 \%$ & $2.1 \mathrm{e}-2(2.8)$ & $72 \%$ & $1.9 \mathrm{e}-2(3.1)$ & $68 \%$ & $1.9 \mathrm{e}-2(3.3)$ \\
\hline $8 \%$ & $-4.9 \mathrm{e}-3(-0.6)$ & $28 \%$ & $-1.2 \mathrm{e}-2(-2.2)-2.2$ & $33 \%$ & $-1.4 \mathrm{e}-2(-2.5)$ \\
\hline $51 \%$ & $3.8 \mathrm{e}-3(4.4)$ & $77 \%$ & $8.7 \mathrm{e}-3(7.0)$ & $78 \%$ & $7.8 \mathrm{e}-3(8.2)$ \\
\hline $49 \%$ & $-7.6 \mathrm{e}-3(-4.5)$ & $23 \%$ & $-5.4 \mathrm{e}-3(-6.4)$ & $22 \%$ & $-4.7 \mathrm{e}-3(-10)$ \\
\hline
\end{tabular}

Table 3.1: Frequency and magnitude of eddy anomalies. The percent of cyclones (left table) and anticyclones (right table) with positive or negative anomalies is reported along with the mean values of anomalies exclusively from eddies with a + or - anomaly. In parenthesis the mean of the normalized anomalies, which is expressed as a percentage of the co-located climatology. Statistics are reported for all Southern Ocean eddies in addition to two subsets delineated based on size (Large: $L_{S}>50 \mathrm{~km} \&$ Amplitude $>5 \mathrm{~cm} \mid$ Small: $L_{S}<50 \mathrm{~km} \&$ Amplitude $<10 \mathrm{~cm}$ ) and background mixing (Deep: $M L D_{\text {clim }}>$ $100 \mathrm{~m}$ | Shallow: $M L D_{\text {clim }}<100 \mathrm{~m}$ ). The number (and \%) of individual realization in each subset is provided above in addition to the number of unique eddy tracks that they come from. Note that not all realizations in a given track are represented in a particular subset.

-1in-1in 
Chapter 4

The SIMUlated BIOLOGICAL RESPONSE TO SOUTHERN OCEAN EDDIES VIA BIOLOGICAL RATE MODIFICATION AND PHYSICAL TRANSPORT 


\begin{abstract}
Surface chlorophyll anomalies have been widely observed in mesoscale eddies and are thought to dominate variability in the global chlorophyll field. Mesoscale eddies can induce anomalous chlorophyll both by modifying in-situ biological rates in addition to physically transporting biomass vertically and horizontally. Different mechanisms can induce anomalies of different sizes in different directions depending on the characteristics of the eddy and its background environment. Variability in the relative role of these processes, and thus the mechanistic underpinning of mesoscale variability, is hitherto largely under-constrained, particularly in the Southern Ocean, where coherent eddy structures are abundant but remote sensing observations are challenging at high latitudes. We examine the structure and drivers of anomalous biomass in Southern Ocean eddies tracked in a global, multi-year, eddy-resolving, 3-D ocean simulation of the Community Earth System Model. We explicitly examine how simulated cyclones and anticyclones differentially modify phytoplankton division rates, loss rates, and ultimately net population growth rates while simultaneously transporting biomass via lateral stirring and trapping as well as vertical mixing and advection. On average, anticyclones typically induce positive net population growth rate anomalies, mix biomass anomalously downward through deeper mixed layers, and advect biomass anomalously upward via eddy-induced Ekman upwelling. The opposite is true in cyclones. The effects of stirring clearly dominate trapping and vary with the strength of the background chlorophyll gradient. Variability in the net effect of these processes is addressed in terms of eddy induced biomass anomalies, both at the surface and integrated across the water column, and is compared to observations. We find that anomalous biomass is largely averaged out across the basin scale but can exist in excess of $10-20 \%$ of background levels at the regional scale, consistent with observation. Moreover, we find a strong seasonality in the size and direction of regional anomalies and the processes that drive them. In the south Pacific Antarctic Circumpolar Current, surface biomass anomalies in cyclones are positive during the winter, but negative and larger in magnitude during the spring. These anomalies are driven first by dilution in the early winter, then by biological rates in the late winter and spring, before a combination of lateral stirring and eddy-induce Ekman pumping maintain weak negative anomalies throughout the remaining quiescent summer and fall months. Within the period of rate-based control, the relative influence of anomalous division rates and grazing rates on net population growth varies. That is, simulated eddies modify biological growth rates from the bottom-up as well as the top-down.
\end{abstract}




\section{Key Points}

- Net population growth rates are generally reduced in cyclones and inflated in anticyclones, however a seasonal reversal is evident in deep mixing ACC waters.

- Anomalous net population growth rates in eddies are predominately driven from the bottom-up, in that they are well coupled to anomalous division rates. However, a strong top-down control during deep mixed layer shoaling, indicated by strong anomalous grazing rates, can decouple anomalous net population growth from anomalous division rates

- Anomalous diabatic mixing and eddy-induced Ekman transport modify the vertical profile of biomass in competing directions. The Ekman flux is typically smaller in magnitude, but similar in size during shallow mixing summer and fall months.

- In ACC cyclones, particularly in the south Pacific, both surface biomass and depthintegrated biomass are inflated in late winter but depressed in spring. In the quiescent summer and fall surface biomass remain elevated but depth-integrated biomass is depressed. The opposite is true in Anticyclones.

- The seasonal cycle of anomalous surface biomass in simulated ACC eddies is driven by dilution in the early winter, biological mechanisms in the late winter and spring, and physical transport via lateral stirring and eddy induced Ekman-pumping in the quiescent summer and fall months.

\subsection{Introduction}

Surface chlorophyll anomalies in mesoscale eddies have been observed across the global ocean [Frenger et al. 2018; Gaube et al. 2014a; Large 1998; McGillicuddy et al. 2007] and are thought to dominate global spatio-temporal variability in phytoplankton distributions [Doney et al. 2003; Glover et al. 2018]. The mechanisms driving this variability appear to be highly variable in both space and time and are hitherto largely under-constrained [Gaube et al. 2014b]. The Southern Ocean, in particular, is characterized by strong mesoscale activity [Meredith 2016] and abundant closed, rotating, eddy structures [Frenger et al. 2015] but has not been the focus of most mesoscale studies to date (i.e. Chelton et al. [2011a] and Gaube et al. [2014b]), in part because of the challenges associated with high latitude remote sensing [Ruddick et al. 2014]. Better understanding the mechanistic underpinning of eddy modified phytoplankton populations will help constrain the Southern Ocean's critical role in global biogeochemical cycling [Marinov et al. 2008; Siegel et al. 2014] and atmospheric carbon uptake [Hauck et al. 2015]. 
It difficult to fully constrain the mechanisms driving eddy-induced biogeochemical anomalies because they modify the environment in a number of different, often competing, ways [McGillicuddy 2016]. For instance, Southern Ocean cyclones, which induce a cold sea surface temperature anomaly $\left(-S S T^{\prime}\right)$ and negative sea surface height anomaly $\left(-S S H^{\prime}\right)$, simultaneously trap water at their core as they propagate, stir water around their periphery as they rotate counterclockwise, stratify the water column as they dome isopycnals [Dufois et al. 2014; Hausmann et al. 2017], and induce Ekman-downwelling as surface currents modify the relative wind stress [Dewar and Flierl 1987; Gaube et al. 2014b]. Anticyclones do the opposite. Moreover, the dominance of how any particular mechanism can modify biology is sensitive to local environmental conditions such as the meridional chlorophyll gradient [Frenger et al. 2018] or the depth of the winter mixed layer [Song et al. 2018]. Ultimately, the ways in which an eddy can induce a biomass anomaly can be broken down into vertical and lateral transport mechanisms, which physically move biomass, and rate-based mechanisms, which biologically stimulate or suppress the growth of biomass (see Fig. 4-1).

Vertical transport can induce a surface biomass anomaly via both mixing and advection, but cannot directly affect the depth-integrated biomass inventory. Indirectly, however, the redistribution of biomass can influence rate-based mechanisms and thereby modify the depth-integrated biomass inventory. For example, in cyclones domed isopycnals can increase stratification and lead to anomalously shallow mixed layers, particularly in the winter when background mixing is strong [Dufois et al. 2016; Hausmann et al. 2017]. In turn, reduced dilution across shallower mixed layers can induce anomalously high surface biomass anomalies relative to surrounding waters. Note that the opposite is true for vertically mixed iron transport (see Ch. 3) because unlike biomass concentrations, which are typically highest in the mixed layer, iron has a typical nutrient profile that increases with depth [Johnson et al. 1997] in the iron-limited Southern Ocean [Boyd 2002]. On the other hand, cyclones can depress surface biomass concentrations via eddy-induced Ekman pumping by advecting biomass from the surface as water is down-welled. Eddies can induce Ekman pumping by altering the curl of the relative wind stress associated with rotating surface currents [Gaube et al. 2014a; McGillicuddy 2016], inducing a SST anomaly [Chelton et al. 2004; O'Neill et al. 2010] or modifying the vorticity gradient [Gaube et al. 2014b]. In cyclones the net effect is eddy-induced Ekman-downwelling [Gaube et al. 2014b]. The opposite is true in anticyclones for both mixing and eddy-induced Ekman pumping. Finally, eddy pumping during formation [Falkowski et al. 1991] could theoretically modify vertical advection in the opposite direction of the eddy-induced Ekman flux, but was found to not dominate in this simulation of the Southern Ocean (see Ch. 3).

Eddies can modify biomass laterally by stirring along their periphery, and by trapping at their core. Lateral processes operate by advecting biomass across a horizontal gradient. This 
can modify both surface concentrations and the depth-integrated inventory, provided that the background gradient is large relative to the spatial scale of the eddy $(10-100 \mathrm{~km}$ [Frenger et al. 2015]), and the timescale of advection is faster than the turn-over rate of the local population (days-months) [Abraham 1998]. Stirring is the process by which eddies disturb the background gradient as they rotate, typically with a timescales of days to weeks [Doney et al. 2003; Frenger et al. 2015; Glover et al. 2018; Siegel et al. 2008]. Trapping refers to the process by which nonlinear eddies (i.e those with a rotational speed faster than their propagation speed [Flierl 1981]) trap and transport water at their core as they propagation [Early et al. 2011; Flierl 1981; Lehahn et al. 2011]. Propagation speeds in Southern Ocean eddies are typically an order of magnitude lower than their rotational speeds, meaning that the majority are nonlinear [Chelton et al. 2011b], but also implying that they do not propagate very far before dissipating [Frenger et al. 2015]. In turn, lateral stirring tends to dominate relative to trapping in Southern Ocean observations [Chelton et al. 2011a; Frenger et al. 2018].

Rate-based mechanisms affect biology in eddies from the bottom-up by modifying phytoplankton division rates as well as from the top-down by modifying phytoplankton loss rates (i.e. grazing, mortality and aggregation). In the Southern Ocean division rates are limited by light [Nelson and Smith 1991] and iron [Carranza and Gille 2015]. Eddies can modify depthaveraged light and nutrient limitation by redistributing biomass and iron profiles. For instance, cyclones can reduce light limitation through anomalously shallow winter mixed layers [Song et al. 2018], but can exacerbate nutrient limitation by suppressing the vertical supply of iron [Gaube et al. 2014b]. The opposite is true in anticyclones. The relative effect of these mechanisms was examined extensively in Chapter $\mathbf{3}$ and found to be dominated by the anomalous vertical transport of iron, leading to depressed division rates in cyclones and elevated division rates in anticyclones throughout most of the year. In the Southern Ocean, loss rates are predominately driven by grazing [Deppeler and Davidson 2017; Rohr et al. 2017], which can be modified by eddies as dilution across anomalous mixed layer depths reduces grazing efficiency [Behrenfeld et al. 2013]. The ensuing anomalous net population growth rate in eddies is equal to the difference between anomalous division rates and loss rates.

Competition between physical and biological mechanisms explains why there is such a high degree of spatio-temporal variability in anomalous biomass within eddies throughout the Southern Ocean [Frenger et al. 2018] and world at large [Gaube et al. 2014b]. Nevertheless, we still lack a complete understanding of where and when different mechanisms dominate. As the physical landscape of the Southern Ocean continues to change [Böning et al. 2008; Gille 2002], a better mechanistic understanding of how eddies variably perturb biogeochemistry is required to predict how biology will respond at the mesoscale. Recently, however, some advances have begun to expand our insight into what is driving mesoscale variability in the Southern Ocean phytoplankton populations. 
A particularly interesting mode of variability has been observed [Frenger et al. 2018; Song et al. 2018] and simulated [Song et al. 2018] in the Antarctic Circumpolar Current (ACC), particularly in the south Pacific. Eddies in this region exhibit a seasonal flip in the direction of surface chlorophyll anomalies during the winter, when background mixed layers are deep $(>150 \mathrm{~m})$, implying that seasonal environmental changes can fundamentally reverse how an eddy modifies the biology within it. Frenger et al. [2018] point out that this seasonal reversal can not be explained by either stirring or trapping and hypothesized that it must be attributed to a combination of modifications to light availability, iron limitation and grazing efficiency [Carranza and Gille 2015; Quéré et al. 2016; Smetacek et al. 2004], but lacked the observational capacity to dig deeper. Song et al. [2018] employ a numerical simulation to examine depthintegrated light and iron limitation and conclude that this flip is controlled by eddy-modified light availability, but do not explicitly examine division rates or net population growth rates. Using the same numerical integration employed by Song et al. [2018], it was confirmed in Chapter 3 that this flip is, in fact, associated with a coincident reversal in the direction of division rate anomalies, but the degree to which physical dilution and/or elevated loss rates driven by improved grazing efficiency (i.e. Behrenfeld et al. [2013]) contribute has not been addressed. Moreover, it remains unclear exactly how physical and biological mechanisms interact, variably amplifying and damping one another, to modify biomass throughout the Southern Ocean.

Here we address the combined effects of anomalous division rates, loss rates, vertical mixing and advection, and lateral stirring and trapping on biomass in the simulated Southern Ocean eddies that were identified and tracked in Chapter 3. We examine spatio-temporal variability in both rate-based mechanisms (4.3.1) and physical transport mechanisms (4.3.2) before addressing variability in the ensuing in surface and depth-integrated biomass anomalies (4.3.3). Next, we highlight profiles of depth-resolved seasonal variability within south Pacific ACC eddies, focusing on the relevant biological rates, fluxes, and biomass concentrations that they drive (4.3.4). Finally, we discuss how various mechanisms appear to control this seasonal variability (4.4.1) and consider the bulk biomass anomaly, integrated across the all ACC and Southern Ocean eddies, in the context of observations (4.4.2).

\subsection{Methods}

\subsubsection{Numerical Simulation}

We analyze a global, eddy-resolving, numerical simulation integrated with the ocean [Smith et al. 2010], ice [Hunke and Lipscomb 2008] and biogeochemistry [Moore et al. 2013] components of the the Community Earth System Model (CESM1) that is identical to the simulation em- 
ployed in Chapter 3. The physical oceans component is based on the Parallel Ocean Program version 2 (POP) [Smith et al. 2010], vertical mixing is based on the K-Profile Parameterization (KPP) [Large et al. 1994], sea ice is treated using CICE4 [Hunke and Lipscomb 2008], and biogeochemistry is treated with the Biogeochemical Element Cycle (BEC) model [Moore et al. 2013]. Each component is described in greater detail in Chapter 3.2.1.

Global solutions integrated at a coarser resolution, have been widely validated against global data sets and shown to capture basin-scale spatial distributions in productivity, chlorophyll concentrations [Doney et al. 2009; Moore et al. 2004, 2013], and carbon cycling [Lima et al. 2014; Long et al. 2013; Moore et al. 2013]. More recently, this exact high resolution run has been shown to simulate more realistic Southern Ocean mixed layers [Harrison et al. 2018], compare favorably to global chlorophyll distributions [Harrison et al. 2018] and replicate observations of seasonal variability in the distribution of mesoscale surface chlorophyll anomalies in the ACC [Song et al. 2018].

Within BEC, Phytoplankton carbon biomass, $C_{\text {phyto }}(\mathrm{mmol} C)$, is resolved for three phytoplankton pools, diatoms, small phytoplankton, and diazotrophs. The carbon biomass concentration of each pool, $\left[C_{\text {phyto }}\right]\left(\frac{m m o l C}{m^{3}}\right)$, is independently modified by biological rates terms as it is simultaneously mixed and advected by the physical oceans component.

\section{Rate-based modification of biomass}

Class specific phytoplankton net population growth $\left(\frac{d\left[C_{\text {phyto }}\right]}{d t}\right)$ is governed by a photosynthetic net primary productivity term, $P_{\text {phyto }}\left(\frac{m m o l}{m^{3} d}\right)$, and opposed by a loss term, $L_{\text {phyto }}\left(\frac{m m o l}{m^{3} d}\right)$, such that

$$
\frac{d\left[C_{\text {phyto }}\right]}{d t}=P_{\text {phyto }}-\text { Loss }_{\text {phyto }}
$$

$P_{\text {phyto }}$ is composed of a volumetric specific division rate, $\mu_{\text {phyto }}\left(d^{-1}\right)$, multiplied by the biomass concentration $\left(P_{\text {phyto }}=\mu_{\text {phyto }} *\left[C_{\text {phyto }}\right]\right) . \quad \mu_{\text {phyto }}$ is governed by temperature dependence, multi-nutrient limitation and light availability, and is described in detail in Chapter 3.2.1. Loss $_{\text {phyto }}\left(\frac{m m o l C}{m^{3} d}\right)$ is composed of a volumetric specific loss rate, $l_{\text {phyto }}\left(d^{-1}\right)$, multiplied by the biomass concentration $\left(L_{\text {phyto }}=l_{\text {phyto }} *\left[C_{\text {phyto }}\right]\right) . l_{\text {phyto }}$ is composed of a nonlinear grazing rate, $g_{\text {phyto }}\left(d^{-1}\right)$, a linear mortality rate mort $_{\text {phyto }}\left(d^{-1}\right)$, and a quadratic mortality/aggregation rate, $a g g_{\text {phyto }}\left(d^{-1}\right)$, such that

$$
l_{\text {phyto }}=g r_{\text {phyto }}+\text { mort }_{\text {phyto }}+a g g_{\text {phyto }} .
$$

The linear mortality rate is equal to the class specific biomass concentration multiplied by a 
class specific linear mortality rate $\left(m_{\text {phyto }}\right)$ such that

$$
\text { mort }_{\text {phyto }}=m_{\text {phyto }} *\left[C_{\text {phyto }}\right]
$$

The quadratic mortality/aggregation rate is equal to the minimum of a class specific maximum linear aggregation rate, agg max, phyto $\left(d^{-1}\right)$, multiplied by the class specific biomass concentration and a class specific quadratic mortality rate, quad $d_{\text {phyto }}\left(d^{-1}\right)$, multiplied by the square of the class specific biomass concentration, such that,

$$
a g g_{\text {phyto }}=\min \left(a g g_{\text {max }}, \text { phyto } *\left[C_{\text {phyto }}\right], \text { quad }_{\text {phyto }} *\left[C_{\text {phyto }}\right]^{2}\right) \text {. }
$$

The nonlinear grazing term is governed by a temperature dependent, non-linear function (Holling type III; [Holling 1959]) of the phytoplankton concentration such that zooplankton become more efficient grazers as phytoplankton concentrations increase,

$$
g r_{\text {phyto }}=\mu_{z \text { oo } ; \text { phyto }}^{\text {max }} L^{T}\left(\frac{\left[C_{\text {phyto }}\right]^{2}}{\left[C_{\text {phyto }}\right]^{2}+g_{\text {phyto }}^{2}}\right)\left[Z_{C}\right],
$$

where $\mu_{z o o ; p h y t o}^{\text {max }}\left(d^{-1}\right)$ is the maximum zooplankton specific grazing rate on phytoplankton class phyto, $L^{T}$ is the dimensionless temperature dependency term, $g_{\text {phyto }}$ is the zooplankton class-specific grazing coefficient $\left(\mathrm{mmol} \mathrm{C} \mathrm{m}^{-3}\right)$, and $\left[Z_{C}\right]$ is the zooplankton carbon biomass concentration $\left(\mathrm{mmol} \mathrm{C} \mathrm{m}^{-3}\right)$. The grazing parameterization is explained in greater detail in Chapter 2.2.1.

All together, the volumetric specific net population growth rate, $r_{\Sigma}\left(d^{-1}\right)$ is equal to the difference between the specific division rate and loss rate,

$$
r_{\text {phyto }}=\mu_{\text {phyto }}-l_{\text {phyto }}
$$

\section{Physical transport of biomass}

The physical transport of biomass is dominated by fluxes $\left(\mathrm{mmolC} / \mathrm{m}^{2} / \mathrm{s}\right)$ from horizontal advection $\left(U_{F e}, V_{F e}\right)$, vertical advection $\left(W_{F e}\right)$, and vertical diabatic mixing $\left(M i x_{F e}\right)$. All flux terms are averaged over the 5 day time step over which model output is saved. Vertical fluxes are positive in the upward direction.

\subsubsection{Description of Diagnostic Tracers}

\section{Biomass}

The majority of observations (i.e. Frenger et al. [2018] and Gaube et al. [2014b]) and modelling work (i.e. Song et al. [2018]) has focused on how eddies modify surface populations. Unfor102 
tunately, surface concentrations may not always be a good indicator of the depth-integrated biomass inventory [Rohr et al. 2017] because of dilution during deep mixing [Behrenfeld et al. 2013], biomass that may exist below a shallow mixed layer [Behrenfeld et al. 2013], or the potential if nonuniform profile distributions [McGillicuddy et al. 2007; Siegel et al. 1999]. In addition to considering surface concentrations $\left(\left[C_{p h y t o}\right]_{S}\right)$, here we also focus on depth-integrated biomass inventories $\left(\Sigma C_{\text {phyto }}\right)$, and profiles of depth resolved concentrations $\left(\left[C_{\text {phyto }}\right]\right)$. In all cases biomass is integrated over the two regionally dominate phytoplankton pools, diatoms and small phytoplankton.

\section{Biological rate terms}

Biological rate terms are expressed both as depth resolved rates $(\mu, l, r)$ and populationaveraged rate terms $\left(\mu_{\Sigma}, l_{\Sigma}, r_{\Sigma}\right)$. Population averages are computed by biomass-weighting depth-averaged values as follows,

$$
\begin{aligned}
\mu_{\Sigma} & =\sum_{z=0 m}^{\text {water column }} \sum_{\text {phyto }=\text { sp,diat }} \mu_{z, \text { phyto }} \frac{\left[C_{\text {phyto }}\right]_{z} * h}{\Sigma C_{\text {phyto }}}, \\
l_{\Sigma} & =\sum_{z=0 m}^{\text {water column }} \sum_{\text {phyto }=\text { sp,diat }} l_{z, \text { phyto }} \frac{\left[C_{\text {phyto }}\right]_{z} * h}{\Sigma C_{\text {phyto }}}, \\
r_{\Sigma} & =\sum_{z=0 m}^{\text {water column }} \sum_{\text {phyto }=\text { sp ,diat }} r_{z, \text { phyto }} \frac{\left[C_{\text {phyto }}\right]_{z} * h}{\Sigma C_{\text {phyto }}},
\end{aligned}
$$

where $C_{\text {phyto,z }}$ is the class specific biomass concentration at a given grid cell and depth $\left(\frac{m m o l}{m^{3}}\right), h$ is the height of the grid cell $(m)$, and the $\Sigma C_{p h y t o}$ is the depth-integrated sum of diatom and small phytoplankton biomass $\left(\frac{m m o l}{m^{2}}\right)$.

\section{Vertical transport mechanisms}

In addition to examining depth resolved profiles of the diabatic mixing flux of carbon, $M I X_{C}$ $\left(\mathrm{mmol} C / \mathrm{m}^{2} / \mathrm{s}\right)$, and the vertical advection flux of carbon, $W_{C}\left(\mathrm{mmol} C / \mathrm{m}^{2} / \mathrm{s}\right)$, we also specifically examine the flux into surface ocean $(1-10 m)$. Surface fluxes are expressed as $M I X_{C, S}\left(\mathrm{mmolC} / \mathrm{m}^{2} / \mathrm{s}\right)$ and $W_{C, S}\left(\mathrm{mmol} C / \mathrm{m}^{2} / \mathrm{s}\right)$ respectively. The velocity of eddyinduced Ekman pumping, $\Omega_{E k}(\mathrm{~m} / \mathrm{s})$, is estimated diagnostically from the curl of the relative surface stress and described in detail in Chapter 3.2.2. Note that the supplemental effects of eddy eddy-induced SST anomalies [Chelton et al. 2004; O'Neill et al. 2010] and nonlinear modifications to the vorticity gradient [Gaube et al. 2014b] are not resolved, but are not expected to qualitatively change the direction our estimation of $\Omega_{E k}$ [Gaube et al. 2014b]. 


\section{Lateral transport mechanisms}

It is difficult to prescribe a precise budget to lateral transport mechanisms, as eddies with dynamic, and permeable, boundaries rotate and propagate across the ocean while simultaneously being modified from within by biological rate-based mechanisms and vertical transport. Moreover, it is difficult to establish a baseline for a single eddy as they interact with an abundance of mesoscale features such as filaments, fronts, and other eddies. Instead of trying to quantify the actual anomalous two dimensional horizontal advection in an individual eddy and then partition it into a stirring and trapping component, we estimate the potential for an anomaly to be created by an isolated eddy moving across the climatological field (see 4.2.4 for how climatologies are computed). Following Frenger et al. [2018], the size and direction of this potential anomaly can be constrained based on the size and direction of the meridional biomass gradient as well as the size, polarity, and propagation path of the eddy in question. Trapping and stirring potentials were created for surface biomass as well as the depth-integrated biomass inventory.

Briefly, the trapping potential $(T R A P)$ represents the anomaly that would be created if source water at the eddy's origin was adiabatically trapped and transported across the climatological biomass field. It is computed as difference between the biomass climatology at formation $(t=1)$ and at the location of any ensuing realization $(t=i)$, such that,

$$
\text { Biomass TRAP }=\overline{\text { Biomass }}_{C l i m, t=1}-\overline{\text { Biomass }}_{C l i m, t=i}
$$

The stirring potential $(S T I R)$ represents the anomaly that could be created by rotating (i.e. flipping) the meridional climatological gradient across the length of the eddy's diameter. If an eddy was stationary then this anomaly would average out over its surface profile, however, because Southern Ocean eddies can be assumed to always propagate westward relative to the mean flow, the dipole created by stirring should be asymmetric. This is because the leading

edge modifies undisturbed waters while the trailing edge stirs water that has already been perturbed [Chelton et al. 2011a; Frenger et al. 2018]. In turn, the sign of STIR can be prescribed based on the polarity of an eddy and the direction of the meridional gradient. For instance, a cyclone moving west and rotating counterclockwise across an increasing south to north gradient would induce a negative anomaly (see Frenger et al. [2018]). The estimated magnitude represents an upper bound as there would likely be some degree of averaging out across the eddy profile. 


\subsubsection{Depth Extrapolation}

Some model output must be extrapolated at depth because storage limitations prevent saving the full-depth profiles of all prognostic tracers. In this simulation biomass and all biological rate terms were archived to $150 \mathrm{~m}$. Extrapolation of biomass and biological rate terms is only necessary if the mixed layer depth exceeds $150 \mathrm{~m}$, as biomass can be assumed to drop to 0 below $150 \mathrm{~m}$ provided it is not being actively mixed down from the euphotic zone. In deep mixed layers biomass is assumed to be well mixed [Uitz et al. 2006] and accordingly linearly extrapolated to replicate a uniform profile. Depth extrapolation is identical to Chapter 3 and is described detail in Chapter 3.2.3.

\subsubsection{Anomaly and climatology Fields}

Anomaly and climatology fields were created identically to those described in Chapter 3.2.4. A detailed description of smoothing techniques and justification of filter size can be found there. Briefly, spatial anomaly fields for each relevant variable $\left(v a r^{\prime}\right)$ are computed by removing the mean field $(\overline{v a r})$ from the raw field (var), such that,

$$
v a r^{\prime}=v a r-\overline{v a r}
$$

Mean fields were created by smoothing the raw fields with a 2D low pass loess filter. Threedimensional anomaly fields were computed independently at each depth. Background climatology fields $\left(\overline{v a r}_{C l i m}\right)$ were computed by averaging over the 5-year model run at each time step (5-days) before being smoothed in time and space. Anomaly fields were divided by the corresponding climatology to create normalized fields $\left(v a r^{\prime \prime}\right)$,

$$
\operatorname{var}^{\prime \prime}=\frac{\operatorname{var}^{\prime}}{\overline{v a r}_{C l i m}}
$$

\subsubsection{Eddy identification and tracking}

The complete set of Southern Ocean eddies analyzed here is identical to those analyzed in Chapter 3. Eddies were identified and tracked as closed contours in the sea surface height anomaly $\left(S S H^{\prime}\right)$ field using the parameter-less algorithm originally developed by Chelton et al. [2011b] and adapted by Faghmous et al. [2015]. Each simulated eddy track is composed of a time series of eddy "realizations" identified at 5-day intervals. See Chapter 3.2.5 for

a detailed description of this methodology. The distribution and demographics of observed eddy tracks Chelton et al. [2011b] compare favorably to those of simulated tracks, particularly north of the marginal ice zone (see Chapter. 3.3.1). 
Each data point included in this study has been averaged across the spatial footprint of the corresponding eddy realization. Other studies (i.e. Chelton et al. [2011a], Frenger et al. [2018], Gaube et al. [2014b], and Siegel et al. [2011]) have additionally considered the lateral structure of the anomaly field in eddy composites to infer mechanistic insight from the shape of the anomaly. (e.g. Frenger et al. [2018]). For instance, a pronounced monopole in the absence of an adequately strong or appropriately oriented trapping potential would indicate the presence of biological rate-based mechanisms. We, however, do not need to infer the role of rate-based mechanisms from the shape of the anomaly field as rate terms are explicitly resolved in the simulation. Further, averaging across the breadth of the eddy ensures values are representative of the mean state of all phytoplankton within an eddy and reduces the dimensionality of the data set, thereby easing visualization.

\subsubsection{Eddy Subsets}

In addition to providing a complete Southern Ocean perspective (i.e. Figs. 4-3 - 4-6, C1 - C-12), eddies are also sorted into various subsets on the basis of their size, geography, and environment. Eddies are referred to as large if their radius is greater than $50 \mathrm{~km}$ and their amplitude is greater than $5 \mathrm{~cm}$. Eddies are said to be in the south Pacific if they are found between $80 W^{\circ}<$ Lon $<180 W^{\circ}$ and are south of $40 S^{\circ}$. Note, however, all eddies in this analysis fall south of $40 S^{\circ}$. Eddies are said to fall in the Antarctic Circumpolar Current (ACC) if they are within the $-20 \mathrm{~cm}$ and $-80 \mathrm{~cm} S S H$ contours [Frenger et al. 2018]), defined dynamically by the daily $S S H$ climatology $\left(\overline{S S H}_{C l i m}\right)$.

The south Pacific is isolated during zonal averaging (Figs. 4-2, 4-7, 4-8, 4-10) to highlight the most coherent signal [Song et al. 2018] with the deepest winter mixing. Large eddies in the south Pacific ACC (Fig. 4-9) are further highlighted because very large mixed layer depth anomalies lead to the most dramatic seasonal cycle (see $\mathbf{C h}$. 3). North of the ACC the biogeochemical response to eddies is less consistent and often statistically insignificant (see Fig. 4-8). South of the ACC the response is complicated by seasonal sea ice. Moreover, the distribution and demographics of simulated eddies north the marginal ice zone are in much better agreement with observations than those further south (see Chapter 3.3.1). Note that there are a total of 9087 realizations from 596 unique tracks that qualify as "large eddies in the south Pacific ACC". This subset is identical to that of Fig. 3-10 in Chapter 3. Seasonal

projections of eddy induced anomalies are also provided for the entire Southern Ocean can be found in Appendix C. 


\subsection{Results}

\subsubsection{Rate-based mechanisms}

\section{Division rates}

Throughout most of the year, cyclones exhibit anomalously low population-averaged division rates $\left(-\mu_{\Sigma}^{\prime}\right)$ while anticyclones exhibit anomalously high population-averaged division rates $\left(+\mu_{\Sigma}^{\prime}\right)$. This is clearly evident in the Pacific (Fig. 4-2a, b) as well as across the entire Southern Ocean (Fig. C-1). Eddy-modified division rates are largely fueled by an anomalous advective flux of iron from depth, driven by anomalous eddy-induced Ekman downwelling in cyclones and Ekman upwelling in anticyclones (see Ch. 3). The primary exception occurs during the winter and early spring when the direction of division rate anomalies is reversed in a number of eddies, most typically within the ACC (Fig. 4-2a, b). This flip in the sign of anomalies is associated with eddies that are within deep background mixed layers and have large $M L D$ anomalies (Fig. 4-3a - d). If background mixing is sufficiently deep, winter cyclones can induce substantially shallower mixed layers than surrounding waters (Fig. 4-3a), thereby improving light limitation enough to compensate for a coincident reduction in iron (see Ch. 3), and trigger a seasonal flip in the direction of division rate anomalies (Fig. 4-3c). The opposite is true in anticyclones (Fig. $4-3 b, d$ ). See Chapter 3 for a detailed breakdown of the mechanistic drivers of eddy induced bottom-up controls on phytoplankton division rates and Tab. 4.1 for a seasonal breakdown in the frequency of cyclones and anticyclones with $+\mu_{\Sigma}^{\prime}$

\section{Loss rates}

Anomalous population-averaged phytoplankton loss rates $\left(l_{\Sigma}^{\prime}\right)$ are composed of contributions from eddy-modified grazing $\left(g r_{\Sigma}^{\prime}\right)$, mortality $\left(\operatorname{mort}_{\Sigma}^{\prime}\right)$, and aggregation $\left(a g g_{\Sigma}^{\prime}\right)$, but are dominated by grazing. Distributions of component rate term anomalies across the Southern Ocean (Fig. 4-4), show that anomalous grazing rates are much larger than anomalous aggregation and mortality rates ( $8 X$ and $15 X$ respectively), and comparable to the magnitude of $l_{\Sigma}^{\prime}$. Moreover, anomalous loss rates are substantially better correlated with anomalous grazing rates $(r=.98)$ than either anomalous mortality $(r=.30)$ or aggregation $(r=-.09)$ rates (Tab. 4.2). Accordingly, the spatial-temporal distribution of $l_{\Sigma}^{\prime}($ Fig. C-2) is very similar that of $g r_{\Sigma}^{\prime}$ (Fig. C-3).

Grazing rates in eddies are modified by processes that change the local biomass concentration and thus the grazing efficiency. Anomalous population-averaged grazing rates are in turn positively correlated with anomalous division rates throughout the year $(r=+.69)$, but also 
negatively correlated with the mixed layer depth anomaly in deep mixing winter ACC eddies $(r=+-.51)$. Together, it is clear that anomalous eddy-induced loss rates are controlled by anomalous grazing rates which are primarily modified by the effects of modified cell division rates and dilution on profiled biomass concentrations.

\section{Net population growth rates}

Anomalous population-averaged net population growth rates $\left(r_{\Sigma}^{\prime} ; \mathbf{F i g}\right.$. 4-2c) represent subtle differences between the competing effects of $\mu_{\Sigma}^{\prime}$ (Fig. 4-2a) and $l_{\Sigma}^{\prime}$ (Fig. 4-2b) [Behrenfeld et al. 2013; Rohr et al. 2017], but are, on average, modified in the direction of $\mu_{\Sigma}^{\prime}$. Even though $\mu_{\Sigma}^{\prime}$ and $l_{\Sigma}^{\prime}$ are generally well-coupled throughout the Southern Ocean $(r=.73$; Tab. 4.2) this is possible because the magnitude of $u_{\Sigma}^{\prime}$ typically leads and exceeds that of $l_{\Sigma}^{\prime}$, driving $r_{\Sigma}$ down in cyclones and up in anticyclones. Analyzing the mean seasonal cycle of all eddy-induced rate anomalies (Fig. 4-5a - c) confirms that even though both $\mu_{\Sigma}$ and $l_{\Sigma}$ are depressed in cyclones, the magnitude of $\mu_{\Sigma}^{\prime}$ is larger such that the aggregate effect is a depression of net population growth rates throughout the year. The opposite is true in anticyclones. In both cyclones (Fig. 4-5b) and anticyclones (Fig. 4-5c), the magnitude of $r_{\Sigma}^{\prime}$ peaks coincidentally with the peak in the magnitude of $u_{\Sigma}^{\prime}$, shortly before the background $M L D$ shoals to its annual minimum, all together indicating a strong bottom-up control [Rohr et al. 2017].

In turn, the spatial-temporal distribution of $r_{\Sigma}^{\prime}($ Fig. 4-2e, f; C-6) is generally well aligned with that of $\mu_{\Sigma}^{\prime}$ for most of the year, but notably diverges in the late winter and early spring, particularly within the ACC. Here, deep background mixing and large eddy induced $M L D$ anomalies can trigger a seasonal reversal in the direction of $u_{\Sigma}^{\prime}$ (see Chapter 3.3.4) followed by a decoupling of $u_{\Sigma}^{\prime}$ and $r_{\Sigma}^{\prime}$. This is most evident in the seasonal cycle of large eddies within the south Pacific ACC plotted in Fig. 4-5d-f. First, while the background $M L D$ is deepening in the early winter, the direction of $l_{\Sigma}^{\prime}$ in both cyclones (Fig. 4-2a, 45e) and anticyclones (Fig. 4-2b, 4-5f) flips quickly following the seasonal reversal of $u_{\Sigma}^{\prime}$. The magnitude of $l_{\Sigma}^{\prime}$, however, still lags behind that of $u_{\Sigma}^{\prime}$ leading to a coincident reversal in the direction $r_{\Sigma}^{\prime}$, remaining consistent with a strong bottom-up control. By roughly the time the background mixed layer has reached it's annual maximum, however, the magnitude of $l_{\Sigma}^{\prime}$ surpasses that of $u_{\Sigma}^{\prime}$ and remains in excess as the background $M L D$ begins to shoal. This is possible because of the effects of dilution which drive grazing rates up in cyclones with shallower mixed layers and down in anticyclones with deeper mixed layer. The result is a brief period of increased top-down control during the early spring in which anticyclones with deep background $M L D$ s can exhibit elevated net population growth rates (Fig. 4-3h), despite depressed division rates (Fig. 4-3d), and vice-versa in cyclones (Fig. 4-3g, c). Eventually, by the time the mixed layer has shoaled, a bottom-up control appears to re-emerge and $u_{\Sigma}^{\prime}, l_{\Sigma}^{\prime}$, 
and $r_{\Sigma}^{\prime}$ all remain elevated in anticyclones and depressed in cyclones throughout the summer and fall.

\subsubsection{Physical transport mechanisms}

\section{Vertical Transport}

Large anomalies in the mixed layer depth can modify the vertical transport of biomass by directly inducing an anomalous diabatic mixing flux of biomass $\left(M i x_{C}\right)$. Eddies with anomalously shallow $M L D$ s, typically cyclones (Fig. 4-6a), exhibit an anomalously upward mixing flux of carbon $\left(+M i x_{C}^{\prime}\right)$, while eddies with anomalously deep $M L D$ s, typically anticyclones (Fig. 4-6b), exhibit an anomalously downward mixing flux of carbon $\left(-M i x_{C}^{\prime}\right)$. The magnitude of $M i x_{C}$ increases with the magnitude of $M L D^{\prime}$ but is dampened in eddies with $M L D^{\prime}$ in a direction that is not consistent with theory (i.e. deep mixing cyclones). The anomalous mixing flux in turn modifies the biomass profile by increasing surface biomass in eddies with shallow mixed layers (Fig. 4-6e) and decreasing surface biomass in eddies with anomalous deep mixed layers (Fig. 4-6f). The opposite is true at depth. Regardless of polarity, the size and direction of $M L D^{\prime}$ is consistent with the associated mixing flux (Fig. 4-6a, c) and the profile of the ensuing biomass anomaly (Fig. 4-6e, f). Seasonally, $M L D^{\prime}$ is the largest during the winter and early spring, when background mixed layers are the deepest (Fig. 4-7a, b; C-7). Geographically, $M L D^{\prime}$ is largest and most consistent with theory (i.e. shallow mixing cyclones) within the south Pacific ACC (Fig. 4-7a, b; C-7). Note that the summertime and ice-covered eddies that modify $M L D^{\prime}$ in an unexpected direction are typically small and subject to shallow background mixed layer depths (see Chapter 3.4.1). Together, it is clear that anomalously low surface biomass in wintertime ACC anticyclones is in part driven by dilution. The opposite is true in cyclones.

Eddy-induced Ekman pumping can also directly modify the vertical transport of biomass by inducing anomalous vertical velocities $\left(\Omega_{E k}^{\prime}\right)$; Eddies with anomalously downward $\Omega_{E k}^{\prime}$, typically cyclones (Fig. 4-6c), exhibit an anomalously downward advective flux of carbon $\left(-W_{C}^{\prime}\right)$, while eddies with anomalously upward $\Omega_{E k}^{\prime}$, typically anticyclones (Fig. 4-6d), exhibit an anomalously upward advective flux of carbon $\left(+W_{C}^{\prime}\right)$. The direction of anomalous vertical advection is typically opposite that of the anomalous mixing flux and slightly lower in magnitude. The ensuing biomass anomalies associated with anomalous eddy-induced Ekman pumping are not as strong as seen with mixing. There is, however, a clear increase in biomass at depth as it is advected from the surface in cyclones with $-\Omega_{E k}^{\prime}$ (Fig. 4-6g) and a clear decrease in biomass at depth as it is advected into the surface in anticyclones with $+\Omega_{E k}^{\prime}$ (Fig. 4-6h). The anomaly is not as strong or consistent at the surface where other mechanisms likely complicate the signal. Compared to $M L D^{\prime}$, the size and direction of $\Omega_{E k}^{\prime}$ is much less variable 
in space and time; $\Omega_{E k}^{\prime}$ is constantly negative (downward) in cyclones and positive (upward) in anticyclones (Fig. 4-7c, d; C-8), consistent with the rotation of their surface currents [McGillicuddy 2016]. The magnitude of $\Omega_{E k}^{\prime}$, however, does increase to the south as the ambient wind speed over the ocean increases [Monahan 2006].

\section{Horizontal stirring and trapping}

The potential for eddies to induce anomalous surface biomass by stirring it around their peripheries as they rotate $\left(\left[C_{P h y t o}\right]_{S} S T I R\right)$ is strongest in the spring, and varies dramatically in size and direction with latitude (Fig. 4-7e, f). This variability stems from variability in the size and direction of the climatological biomass gradient. In the south Pacific, large spring blooms in the ACC and marginal ice zone (MIZ) create strong north to south gradients north of the ACC and MIZ with a strong south to north gradient in the valley between them (see Fig. C-9d). Thus, during the spring, westward-propagating cyclones have the potential to induce a large positive anomaly in the north and south of the south Pacific and a large negative anomaly in between (Fig. 4-7e). The opposite is true of westward propagating anticyclones, which stir tracers in the opposite direction as they rotate Fig. 4-7e. See Appendix C, Figure C-9 for complete Southern Ocean distributions.

The potential for eddies to induce anomalous surface biomass by trapping it in their core as they propagate $\left(\left[C_{P h y t o}\right]_{S} T R A P\right)$ is much weaker. The potential influence of trapping is statistically insignificant throughout the much of the year (Fig. $4-7 g, \mathbf{h})$. Where trapping is statistically significant (e.g., the springtime ACC), it generally operates in the same direction as stirring, but at a much lower magnitude. The reason that trapping typically has the potential to induce an anomaly in the same direction as stirring is because cyclones in this simulation have a disproportionate tendency to propagate northward relative to anticyclones. Northward propagation across a meridional gradient should stimulate the same sign anomaly as westward propagation and clockwise rotation across the same meridional gradient (i.e. stirring in cyclones) [Frenger et al. 2018]. The opposite applies to anticyclones, which rotate counter clockwise and disproportionately propagate southward. This logic does not take into account any simultaneous propagation across a zonal gradient, however, in the open Southern Ocean, far from coastal boundary currents, zonal gradients are much weaker than meridional gradients. Overall, the effects of trapping appear dominated by those of stirring. See Appendix C, Figure C-10 for complete Southern Ocean distributions.

\subsubsection{Spatial and seasonal variability in biomass anomalies}

Overall, surface biomass concentrations $\left(\left[C_{P h y t o}\right]_{S}\right)$ are elevated in a similar proportion of Southern Ocean cyclones $(\sim 47 \%)$ and anticyclones $(\sim 50 \%)$. Despite a large degree of spatial 
(Fig. 4-8a, b; C-11) and temporal (e.g. Tab. 4.1) variability, cyclones and anticyclones rarely modify $\left[C_{P h y t o}\right]_{S}$ in the same direction at the same time in the same region. Instead, surface biomass concentrations exhibit a symmetric response in cyclones and anticyclones, with anomalies of similar magnitude but opposite direction. In ACC cyclones, particularly in the deep mixing pacific sector, $\left[C_{P h y t o}\right]_{S}$ is generally elevated during the winter and depressed during the spring, with the largest magnitudes evident in the late winter and early spring (Fig. 4-8a). In terms of phenology, this can shift the spring bloom earlier in cyclones relative to anticyclones. Over the summer and fall $\left[C_{P h y t o}\right]_{S}$ remains depressed but with a much lower magnitude. Further north, $\left[C_{P h y t o}\right]_{S}$ is also elevated during the winter but exhibits a less consistent, and often statistically insignificant, signal in the spring. Further south, cyclones exhibit the inverse seasonal cycle, with depressed $\left[C_{P h y t o}\right]_{S}$ in the winter below the ice and elevated $\left[C_{P h y t o}\right]_{S}$ in the spring as the ice recedes. In all cases, the opposite is generally true of anticyclones (Fig. 4-8b). Detailed distributions of the entire Southern Ocean and the seasonal breakdown in the frequency of positive anomalies are provided in Fig. C-11 and Tab. 4.1 respectively.

During the late winter and spring, the anomalous depth-integrated biomass inventory $\left(\Sigma C_{\text {Phyto }}^{\prime}\right.$; $($ Fig. 4-8c, d; C-12)) is largely consistent with anomalous surface concentrations. This suggests that the effects of anomalous dilution and detrainment either do not dominate the biological response to eddies or induce anomalies that are consistent with the direction of those that can be attributed to other mechanisms. Elsewhere, however, $\Sigma C_{P h y t o}^{\prime}$ and $\left[C_{P h y t o}\right]_{S}$ are not as well aligned. For the majority of the fall and summer, amidst a shallow background mixed layer, surface biomass is slightly depressed in cyclones while depth integrated biomass is slightly inflated. The opposite is true in anticyclones. Depth resolved anomaly profiles are examined in 4.3.4 to help investigate these distinctions.

\subsubsection{Depth resolved seasonal variability}

There is a high degree of seasonal variability evident across the water column in how eddies modify the biomass profile via both physical transport and in-situ rate-based mechanisms. To better understand this seasonal cycle profiles of anomalous carbon fluxes (Fig. 4-9a - d), rates (Fig. 4-9e - j) and concentrations (Fig. 4-9k, l) are averaged across all large eddies in the south Pacific ACC and plotted as a function of their seasonal timing.

Throughout the year, diabatic mixing, driven by mixed layer modification, and vertical advection, driven by eddy-induced Ekman pumping, transport biomass in competing directions (see 4.3.2). During the winter and spring, anomalous vertical transport is dominated by the anomalous vertical mixing flux. Here, anomalously shallow mixed layers in cyclones increase the upward mixing flux of biomass relative to surrounding waters $\left(M i x_{C}^{\prime} ;\right.$ Fig. 4-9a) 
by suppressing dilution. $M i x_{C}^{\prime}$ remains positive during the summer and fall, however, the magnitude decreases to a similar level as the advection flux ( $W_{C}^{\prime}$; Fig. 4-9c). Moreover, anomalous advection is present below the mixed layer where the magnitude of $M i x_{C}^{\prime}$ regresses towards zero. The opposite is true in anticyclones (Fig. 4-9b, d).

As biomass is vertically transported, it is simultaneously modified by in-situ rate-based mechanisms, the strength and direction of which vary throughout the year. At any given depth and time, anomalous net population growth rates $\left(r^{\prime} ; \mathbf{F i g} . \mathbf{4 - 9} \mathbf{i}, \mathbf{j}\right)$ are driven by the difference between anomalous division rates $\left(\mu^{\prime} ;\right.$ Fig. 4-9e, f), which are discussed in detail in Chapter 3, and anomalous loss rates $\left(l^{\prime}\right.$; Fig. 4-9e, f), which are driven primarily by grazing (see 4.3.1) and thus closely coupled to biomass concentrations ([$\left.C_{\text {Phyto }}\right]^{\prime}$; Fig. 4-9e, f). In cyclones $r$ is depressed in the surface ocean throughout the year, but not necessarily throughout the rest of water column. In late fall and early winter $r$ is slightly elevated at depth, with the depth of this transition increasing as winter progresses and the mixed layer deepens. By the $M L D$ maximum in late winter, $r$ is depressed throughout the mixed layer and $r_{\Sigma}$ has reached its annual peak magnitude. As the mixed layer subsequently shoals throughout the spring, $r$ in inflated in waters below the $M L D$ where biomass is detrained. The opposite is true in anticyclones. Depth integrated, population-averaged net population growth rates $\left(r_{\Sigma}^{\prime}\right)$ are discussed in more detail in 4.3.1 and represent the confluence of modifications to local rate terms across the water column and the redistribution of biomass throughout it.

Depth resolved biomass anomalies are driven by contributions from both physical transport and in-situ rate based mechanisms. In cyclones (Fig. 4-9k), biomass is elevated across the mixed layer during the winter when mixed layer depths are anomalously shallow. Biomass below the mixed layer depth appears to be anomalously low, but the depth-integrated biomass inventory remains elevated, particularly in the late winter when anomalous surface concentrations are beginning to peak. Shortly thereafter the climatological mixed layer begins to shoal and the biomass anomaly flips direction, becoming anomalously low, both at the surface and integrated across the water column. By late spring biomass remains depressed in the upper ocean but is elevated at depth. This vertical gradient in the anomaly field remains through the summer and fall but decreases in magnitude as the year progresses. The net result is slightly depressed surface concentrations but slightly elevated inventories throughout the summer and fall. The opposite cycle is evident in anticyclones (Fig. 4-9i). 


\subsection{Discussion}

\subsubsection{Seasonal variability in relative contribution from various mechanisms}

Throughout space and time, different mechanisms dominate in their ability to drive the development of anomalous biomass, both in terms of surface concentration and depth-integrated inventory. To better understand the relative contribution of each mechanism, it is helpful to examine spatio-temporal variability in the correlation between anomalous biomass and the underlying mechanisms that drive it (Fig. 4-10). The Hövmoller distributions depicted in Fig. 4-10 are isolated to the south Pacific and include bins identical to those in Fig. 4-2, 4-7, and 4-8. Together, it is possible to infer when and where different mechanisms dominate eddy-induced biomass anomalies. Seasonal variability in the mechanistic underpinning of cyclonic anomalies are described below in the context of our results and previous findings. Similarly located anticyclones consistently respond in a symmetric manner. We focus on the south Pacific ACC (see 4.2.6), as it has been observed to have the most striking seasonal cycle [Frenger et al. 2018; Song et al. 2018]. Other regions to the north, south, or in the Atlantic are briefly addressed below.

\section{Deep mixing period from late fall through early winter - south Pacific ACC}

During the late fall and early winter, as the background mixed layer deepens, dilution appears to be the most important mechanism driving anomalous biomass in cyclones. As cyclonic mixed layers remain shallow relative to surrounding waters, $\left[C_{p h y t o}^{\prime}\right]_{S}$ increases disproportionately relative to $\Sigma C_{\text {phyto }}^{\prime}(\mathbf{F i g} . \mathbf{4 - 8} \mathbf{a}, \mathbf{c})$ and the correlation between the two decreases relative to the spring and early summer (Fig. 4-10c) This slight decoupling of $\left[C_{\text {phyto }}^{\prime}\right]_{S}$ and $\Sigma C_{\text {phyto }}^{\prime}$ is consistent with a dilution-driven mechanism [Behrenfeld et al. 2013]. Moreover, $\left[C_{p h y t o}^{\prime}\right]_{S}$ is positively correlated with the anomalous mixing flux of biomass into the surface ocean $\left(M i x_{C, S}^{\prime}\right.$; Fig. 4-10c). Even though anomalous net population growth rates have begun to increase (Fig. 4-2e), the strength of the correlation between $\left[C_{p h y t o}^{\prime}\right]_{S}$ and $M i x_{C, S}^{\prime}$ is stronger than that of any other mechanism, including time-lagged rates. In the early winter, it appears that the flip in the direction of surface chlorophyll anomalies observed by Song et al. [2018] is actually driven by dilution.

\section{Late winter shoaling and the spring bloom - south Pacific ACC}

By the late winter, once the mixed layer has reached its annual maximum, rate-based mechanisms appear to take on a more dominant role. Here, $\left[C_{\text {phyto }}^{\prime}\right]_{S}$ remains positive, increasing in magnitude (Fig. 4-8a), while $\Sigma C_{\text {phyto }}^{\prime}$ becomes both larger and more consistently statistically significant (Fig. 4-8c). These anomalies are not likely to stem from stirring, which is expected 
in observations [Frenger et al. 2018] and our simulation (Fig. 4-7a) to induce anomalies in the opposite direction, or dilution, which loses relevance once mixed layer deepening shuts down. Instead, the correlation between $\Sigma C_{\text {phyto }}^{\prime}$ and time lagged (1 month) population-averaged net population growth rates $\left(r_{\Sigma}^{\prime}\right)$ is now positive (Fig. 4-10e). This is consistent with elevated midwinter division rates and net population growth, and a strong bottom-up control, seasonally supporting the hypothesis developed by Song et al. [2018].

As winter turns to spring and the mixed layer begins to shoal, the direction of $\left[C_{p h y t o}^{\prime}\right]_{S}$ and $\Sigma C_{\text {phyto }}^{\prime}$ begins to flip sign, becoming negative in the early spring. This is consistent with a late winter flip in the direction $r_{\Sigma}^{\prime}$ (Fig. 4-2e) and positive correlation between time-lagged $r_{\Sigma}^{\prime}$ and $\Sigma C_{\text {phyto }}^{\prime}$, suggesting that rate-based mechanisms still dominate. Note, however, that the reversal of $r_{\Sigma}^{\prime}$ precedes the reversal of $\mu_{\Sigma}^{\prime}$ (Fig. 4-2a) and coincides with a coincident elevation in the magnitude of $l_{\sigma}^{\prime}$ (Fig. 4-2c), indicating that while biological rate-based mechanisms dominate, they are now subject to a top-down control. Similar periods of top-down control associated with deep mixing have been observed in the North Atlantic [Behrenfeld et al. 2013] and simulated in the Southern Ocean [Rohr et al. 2017].

By the middle of spring the mixed layer has shoaled completely (Fig. 4-9) and $\Sigma C_{\text {phyto }}^{\prime}$ and $\left[C_{\text {phyto }}^{\prime}\right]_{S}$ are well coupled (Fig. 4-10f) and substantially depressed (Fig. 4-8a, c). While rate-based mechanisms can explain this seasonal transition it is important to note that $\left[C_{\text {phyto }}^{\prime}\right]_{S}$ is also now positively correlated with the stirring potential (Fig. 4-10a), suggesting an increasingly important role for lateral transport mechanisms.

\section{Quiescent summer and early fall - south Pacific ACC}

After the spring bloom, the magnitudes of $r_{\Sigma}^{\prime}, \Sigma C_{\text {phyto }}^{\prime}$, and $\left[C_{\text {phyto }}^{\prime}\right]_{S}$ are largely diminished (Fig. 4-2e; 4-8a, c respectively), and physical transport mechanisms appear to dominate during the quiescent summer and fall months. The correlation between $\Sigma C_{\text {phyto }}^{\prime}$ and time-lagged $r_{\Sigma}^{\prime}$ is now negative, while the correlation between $\left[C_{\text {phyto }}^{\prime}\right]_{S}$ and various physical transport mechanisms becomes positive. Negative $\left[C_{p h y t o}^{\prime}\right]_{S}$ can be explained by both lateral stirring (Fig. 4-10a) and the anomalous downward advection of biomass out of the surface (Fig. 4-9c, 4-10c). Trapping is also generally positively correlated with $\left[C_{p h y t o}^{\prime}\right]_{S}$ but has a much weaker magnitude than stirring and is often statistically insignificant (Fig. 4-7b). Further, because our estimation of trapping assumes perfect efficiency the actual effects of trapping are likely even weaker. In reality, observed and simulated eddies likely exchange some degree of water with their surroundings as they propagate, even if they are nonlinear [Beron-Vera et al. 2013; Haller 2015]. The dominance of stirring relative to trapping is consistent with observational estimates of stirring and trapping throughout the open ocean far from coastal regions where eddies can pinch off from boundary currents across strong zonal chlorophyll 
gradients [Frenger et al. 2018].

Interestingly, while reductions to $\left[C_{\text {phyto }}\right]_{S}$ agree with observations [Frenger et al. 2018], $\Sigma C_{\text {phyto }}$ is elevated, leading to a prevailing negative correlation between $\left[C_{\text {phyto }}^{\prime}\right]_{S}$ and $\Sigma C_{\text {phyto }}^{\prime}$ (Fig. 4-10f). It is not entirely clear what accounts for this coincident increase in depthintegrated biomass. Neither rate-based mechanisms nor lateral mechanisms can adequately explain this distinction. Regarding rates, net population growth rates are depressed throughout the summer and the time lagged correlation between $r_{\Sigma}^{\prime}$ and $\Sigma C_{\text {phyto }}^{\prime}$ is negative (Fig. 4-10e). Regarding lateral transport, stirring is expected to induce negative anomalies in $\left[C_{\text {phyto }}^{\prime}\right]_{S}$ (Fig. 4-7a) and $\Sigma C_{\text {phyto }}^{\prime}$ (not shown), while trapping is generally not statistically significant in the summer and fall (Fig. 4-7g). Note, however, that our estimation of stirring, which is based on the climatological gradient (see 4.2.2) may not be appropriate during the summer and fall when the climatological gradient is weak (Fig. C-11, C-12). The lone remaining explanation is vertical transport processes. An anomalously downward advection flux, driven by eddy-induced Ekman downwelling, can explain the suppression of surface biomass and inflation of biomass at depth (Fig. 4-6g, 4-9k), but should not locally effect the depth-integrated biomass anomaly beyond its effect on anomalous net population rates, which are negative. It is possible, however, that elevated biomass at depth has remained since the late spring, when there was a strong anomalously downward flux of biomass out of densely populated surface waters (Fig. 4-9c) associated with a dramatic increase in the anomalous biomass at depth (Fig. 4-9k). Shallow mixed layers during the summer and fall could allow deep biomass anomalies to persist and regenerate at the bottom of the euphotic zone without being mixed to the surface where $\left[C_{p h y t o}^{\prime}\right]_{S}$ remains negative (Fig. $\left.\mathbf{4 - 9 k}\right)$. It is important, however, to temper the relevance of this proposed pathway with a reminder that the magnitudes of $\Sigma C_{\text {phyto }}^{\prime}$ and $\Sigma C_{\text {phyto }}$ are both very small relative to the spring.

\section{Brief note on other regions}

North of the ACC, the seasonal mixing cycle is weaker (Fig. 4-7a, b) and the direction of potential stirring anomalies is reversed in the spring (Fig. 4-7e, f), leading to $\left[C_{\text {phyto }}^{\prime}\right]_{S}$ with lower magnitude, more variable direction, and a less coherent mechanistic underpinning. In the summer, however, we see evidence of a meridional flip in the direction of $\left[C_{\text {phyto }}^{\prime}\right]_{S}$ (Fig. 4-7a, b; C-11) driven by stirring (Fig. 4-7e, f; C-9) which agrees with observations by [Frenger et al. 2018]. Further comparison is difficult as our domain does not extend quite as far north.

South of the ACC, the seasonality of the anomaly field is reversed, with $\left[C_{\text {phyto }}\right]_{S}$ depressed in winter cyclones and $\left[C_{\text {phyto }}\right]_{S}$ elevated in spring cyclones (Fig. 4-8a). This change can largely be accounted for by stirring (Fig. 4-10a) but also may stem from a new set of 
dynamics associated with sea ice and how eddies modify the water below it. For instance, the direction $M L D^{\prime}$ is often reversed in ice covered eddies relative to the open ocean (see

Chapter 3.4.1). The importance of ice is supported by the phenology of $\left[C_{p h y t o}^{\prime}\right]_{S}$, which appears tied to the seasonal sea ice cycle. The timing of the flip in direction of $\left[C_{p h y t o}^{\prime}\right]_{S}$ is delayed further south (Fig. 4-8a, b) and appears to follow the ice edge (black contour). These processes are interesting but were not thoroughly investigated because simulated and observed eddy demographics are not as consistent below the ice as they are further north (see Chapter 3.3.1) and observations of chlorophyll at such high latitudes are spotty [Frenger et al. 2018].

The zonal structure described in the south Pacific is not as consistent elsewhere in the Southern Ocean (see Fig. C-1 - C-12), as it can be complicated by a northward migration of the ACC, coastal and shelf waters, weaker winter mixing, and regions heavily influenced by other sources of iron. Biomass anomalies in the Patagonian Shelf for example, are very large and highly variable (Fig. C-11, C-12). This is likely because iron availability is generally highest at the surface and more zonally variable due to strong aeolian and sedimentary sources of iron [Moore and Braucher 2008]. This can reverse the effect of deep mixing on the iron supply (Fig. B-3), increase the strength of lateral transport (Fig. C-9, C-10) and permit for some of the largest summertime biomass anomalies simulated in the Southern Ocean (Fig. C-11, C-12). Biomass anomalies are similarly high and complicated in other regions with exogenous sources of iron, such as the Kerguelen Plateau or southeast of Australia [Moore and Braucher 2008]. Further from shore, in the open ocean Atlantic sector, winter mixed layer depths are much smaller (se Fig. 3-5), leading to some of the weakest springtime net population growth rate (Fig. C-6) and biomass (Fig. C-11, C-12) anomalies seen throughout the Southern Ocean. Together, it made the most sense to address the Pacific basin in detail, but projections of the entire Southern Ocean are provided for reference in Appendix B and Appendix C.

\subsubsection{Comparison of simulated and observed basin scale biomass distribu- tions}

This simulation and observations [Frenger et al. 2018] agree that the rectified basin scale anomalous biomass in eddies is an order of magnitude smaller than regional anomalies. Overall, at any given time in the simulation, integrated across the entire Southern Ocean there is a total of 743 anomalous tonnes of depth-integrated carbon biomass in simulated cyclones and -1765 anomalous tonnes in anticyclones (see Tab. 4.3a for a detailed breakdown of spatially integrated anomalies and their size relative to background levels). Together, that averages out to -1022 tonnes of anomalous biomass in all simulated Southern Ocean eddies. Qualitatively, 
this is consistent with observation of a net negative anomaly in anticyclones that dominates a net positive anomaly in cyclones [Frenger et al. 2018]. Quantitatively, the simulated signal has a slightly lower magnitude than than observations. In the model, integrated anomalous biomass is roughly half a percent of the total, spatially integrated background biomass in the corresponding set of Southern Ocean eddies, compared to $1-4 \%$ in the observations [Frenger et al. 2018]. Note, however, that observations only considered eddies with a radius greater than $50 \mathrm{~km}$ that occur north of $65^{\circ} \mathrm{S}$, which has the effect of excluding small eddies with typically smaller anomalies and reducing the amount of averaging out that occurs in southern Antarctic waters where there is a pronounced shift in phenology [Thomalla et al. 2011]. It is possible that these differences could also stem from the effects of photoacclimation and community composition complicating the comparison between chlorophyll in observations and carbon biomass in the simulation.

Qualitatively, variability in the distribution of simulated biomass anomalies is also well aligned with observations [Frenger et al. 2018]. Both exhibit pronounced spatio-temporal variability yet consistently show that cyclones and anticyclones perturb biomass in opposite directions, even if those directions change throughout space and time. Spatially, the structure of variability in the anomaly field is largely zonal which agrees with variability in observed eddy-induced anomalies [Frenger et al. 2018; Gaube et al. 2014a] as well as the general structure of the climatologic chlorophyll field [Ardyna et al. 2017]. Anomalies in the Atlantic, however, are generally weaker and less coherent than in the Pacific in both this simulation and observations [Song et al. 2018]. Deviations from this structure are most common in coastal and shelf waters where other processes become increasingly relevant, such as the transport of near shore iron [d'Ovidio et al. 2015; Xiu et al. 2011]. Temporally, the simulation agrees that seasonal variability is the strongest south of the subtropics, particularly in the ACC. Further, observational work by [Song et al. 2018] agrees that this seasonal cycle is the most pronounced in south Pacific ACC. South of the ACC both exhibit a seasonal cycle with phenology shifted later in the year.

Quantitatively, the total integrated simulated Southern Ocean biomass anomaly is seasonally most pronounced in the spring, particularly October, when cyclones and anticyclones account for -9928 and +10521 tonnes of anomalous carbon respectively, or roughly $2 \%$ of background biomass. Regionally, isolating environments which modify biomass in a more consistent direction reduces the effect of averaging out across strong spatial variability. For instance, when considering only ACC eddies (Tab. 4.3b) the magnitude of regionally integrated anomalous biomass actually increases in some months relative to the same months integrated across the entire Southern Ocean despite the number of eddies decreasing dramatically. Frenger et al. [2018] observed that the magnitude of anomalies can often exceed $10 \%$ of background levels over certain regional subsets. This is consistent with values of $10-20 \%$ 117 
found in simulated eddies in the south Pacific ACC during the winter.

Still, compared to the total amount of biomass in the Southern Ocean, these numbers are not large. Even in the subsets with the strongest anomalies, the total spatially integrated anomalous biomass rarely exceeds 2 hundredths of a percent (.02\%) of total Southern Ocean biomass. This stems from not only the size of the spatial footprint of eddies ( $\sim 1 \%$ of the total domain) and the size of their anomalies, but also from the pronounced effect of spatial variability in the direction of anomalies, even in eddies of the same polarity. Note, however, that this simulation does not resolve sub mesoscale processes that could enhance the rectified iron supply [Brannigan 2016; Mahadevan et al. 2008] or consider non-local stirring effects [d'Ovidio et al. 2015] that could precondition downstream blooms [Ardyna et al. 2017]. Moving forward, much care should be taken when making inferences about the direction of integrated biomass anomalies in Southern Ocean eddies as the signal can easily, but not necessarily, be averaged out at the basin scale. Understanding variability in the mechanisms described here should help translate how regional anomalies can be scaled up.

\subsubsection{Notes on export}

Global distributions of export production (EP) in coarser runs of CESM are in general agreement with observations [Harrison et al. 2018; Siegel et al. 2014]. In this high resolution eddy resolving run, globally integrated $\mathrm{EP}$ has been shown to be reduced by $2 \%$ relative to a coarser, $1^{\circ}$ control run. Locally, however, the change in export production was found to be regionally variable, and often in excess of $50 \%$ of the control run. Specifically in the Southern Ocean export was found to increase on the northern flank of the ACC but decrease dramatically in the Patagonian Shelf. Changes in EP were found to be predominately driven by changes to net primary production (NPP), rather than export efficiency [Harrison et al. 2018]. Note, however, that these results are not exclusively linked to eddies as the higher resolution simulation also resolves other mesoscale features and improves mixing dynamics. The reduction in export in the Patagonian Shelf, for example, is tied to improved resolution of coastal jets which block off the supply of nutrients to offshore production.

Specifically considering eddies, anomalous export is tied to eddy-induced changes in NPP and export efficiency. Both are likely to vary with the mechanisms that drive anomalous biomass in eddies. For instance, if biomass anomalies are induced strictly by lateral transport rather than rate-based mechanisms, as seen in much of the summer and fall (see 4.4.1), then it is unlikely that this would induce anomalous export as NPP is not being modified at the regional scale. On the other hand, eddies in which biomass is inflated by increased division rates, as seen in spring ACC anticyclones (see 4.4.1)), are likely to also experience increased export. 
Variability in anomalous eddy-induced export efficiency, defined as the ratio of EP to NPP is more complicated. For instance, if NPP is being driven by iron provided by anomalous eddy-induced Ekman pumping it is likely this will fuel diatom productivity which is typically associated with faster sinking silica shells and increased export efficiency [Armstrong et al. 2001; Michaels and Silver 1988]. On the other hand, if anomalous NPP is fueled by deeper mixed layers with surplus iron but lower light, a shift to Phaeocystis might be expected (see Chapter 6.2.1) which might decrease export efficiency [Laws et al. 2000]. Further, deeper mixing leads to longer residence times in the surface ocean and decreases the chance particulate organic matter will escape to the deep before being recycled. Without explicitly quantifying anomalous EP, which was not the focus of this work but could be focus of future studies, it is difficult to precisely describe how eddies will modify export, but it is safe to say that the effect will be dictated by a suite of competing mechanisms and subject to high degree of spatial-temporal variability.

\subsection{Conclusions}

Simulated Southern Ocean eddies can modify biomass via several pathways, the dominance of which is subject to a great deal of regional and seasonal variability. From a biological perspective, biomass inside of anticyclones is typically subject to elevated net population growth rates driven from the bottom-up by elevated division rates. If mixed layer anomalies are large enough, however, the direction of anomalous division rates can flip during mixed layer deepening and the role of grazing-based top-down controls can increase during mixed layer shoaling. From a physical perspective the effects of lateral transport are dominated by stirring rather than trapping, but the direction of anomalies that can be induced by stirring are subject to the structure of the local background biomass gradient. Vertical transport is dominated by a diabatic mixing flux in the winter which dilutes surface concentration in anticyclones, but is also opposed by eddy-induced Ekman upwelling, which acts in the opposite direction throughout the year. In the summer and fall the Ekman flux is similar in size to the mixing flux and is not limited to transport within the mixed layer. Cyclones consistently exhibit a symmetric response to anticyclones, regardless of seasonal or regional variability. Together, the ensuing structure of eddy-induced biomass anomalies is highly variable across time and

space and can easily be averaged out at the basin scale, even when exclusively considering eddies of the same polarity.

On a regional scale, however, there is a pronounced seasonal cycle in cyclones within the south Pacific ACC. In the late fall and early winter surface biomass is elevated because anomalously shallow mixed layers do not dilute biomass as much as in surrounding waters. By mid to late winter, biological rate-based mechanisms take control and elevated population aver119 
aged division rates drive surface, and depth-integrated, biomass up. By late winter, however, anomalously high loss rates, elevated by improved grazing efficiency in a less diluted water column, surpass anomalous division rates and population averaged net population growth rates are depressed. In turn, when combined with the complimentary effects of stirring, biomass is depressed throughout the spring. By summer and fall anomalous net population growth is low and anomalous biomass is driven by a combination of lateral stirring and eddy-induced Ekman pumping, leading to weak negative surface anomalies but positive depth integrated anomalies.

Previous observational and modelling work have identified this cycle and attributed it predominately to anomalous division rates. Like all bio-physical interactions at the mesoscale in Southern Ocean, it is not quite that simple. Moving forward it is important to remember that polarity alone is not a particularly useful predictor of anomalous biomass without information about local environmental conditions that are likely to change in space and time. Moreover, the source of an observed eddy-induced biomass anomaly must be informed by a deeper mechanistic understanding. For instance, anomalously high surface chlorophyll in eddies is not indicative of elevated net primary productivity if it was induced by dilution or lateral trans-

port. As the physical landscape of the Southern Ocean changes these distinctions will be critical in predicting its influence on net primary productivity and larger scale biogeochemical and climate cycles.

\subsection{Acknowledgments}

The CESM project is supported by the National Science Foundation and the Office of Science (BER) of the U.S. Department of Energy. Computing resources were provided by the Climate Simulation Laboratory at NCAR's Computational and Information Systems Laboratory (CISL), sponsored by the National Science Foundation and other agencies. This research was enabled by CISL compute and storage resources. TR and SCD acknowledge support from the National Aeronautics and Space Administration Ocean Biology and Biogeochemistry Program (NNX14AL86G) and the National Science Foundation Polar Programs award 1440435 (Antarctic Integrated System Science) to the Palmer LTER program. Please contact trohr@mit.edu for further questions or to access to data. 


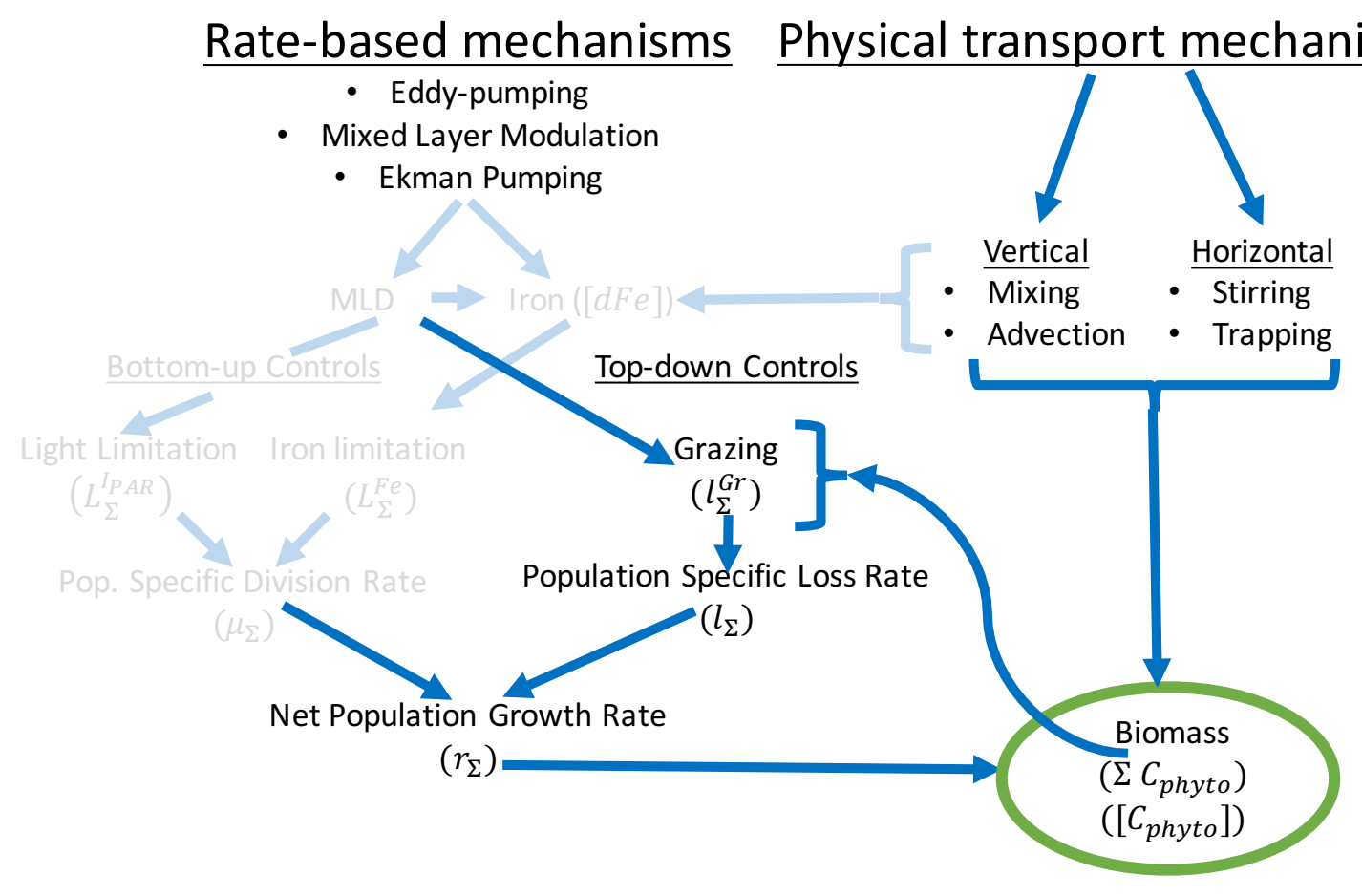

Figure 4-1: Pathways for eddy influence on biomass. Idealized flow chart for how eddies can induce a biogeochemical response. Rate-based mechanisms (on the left) interact with physical transport mechanisms (on the right) to ultimately dictate the net effect on biomass. Highlighted pathways and tracers are examined here. The rest are examined in Chapter 3. 

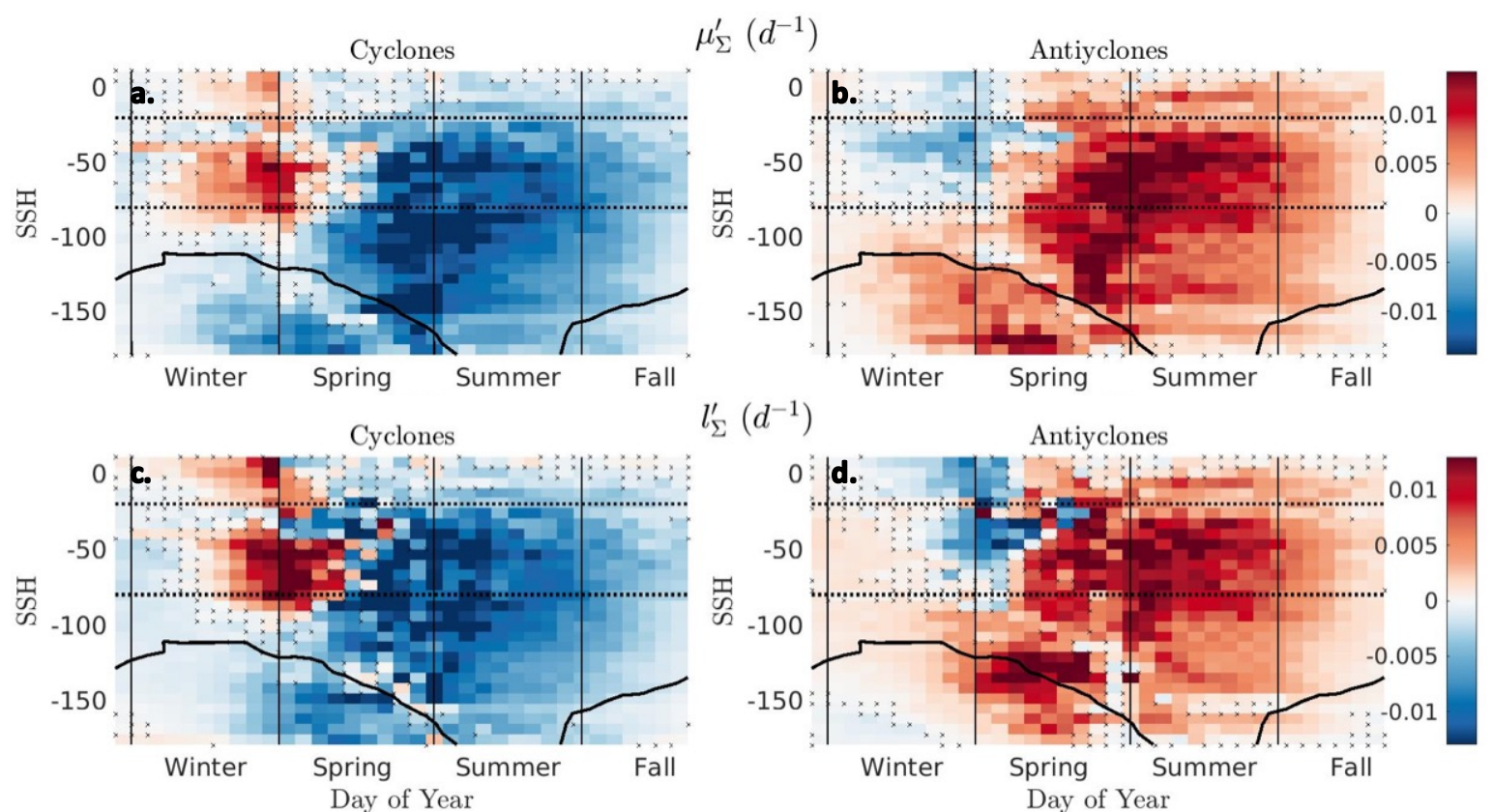

$l_{\Sigma}^{\prime}\left(d^{-1}\right)$
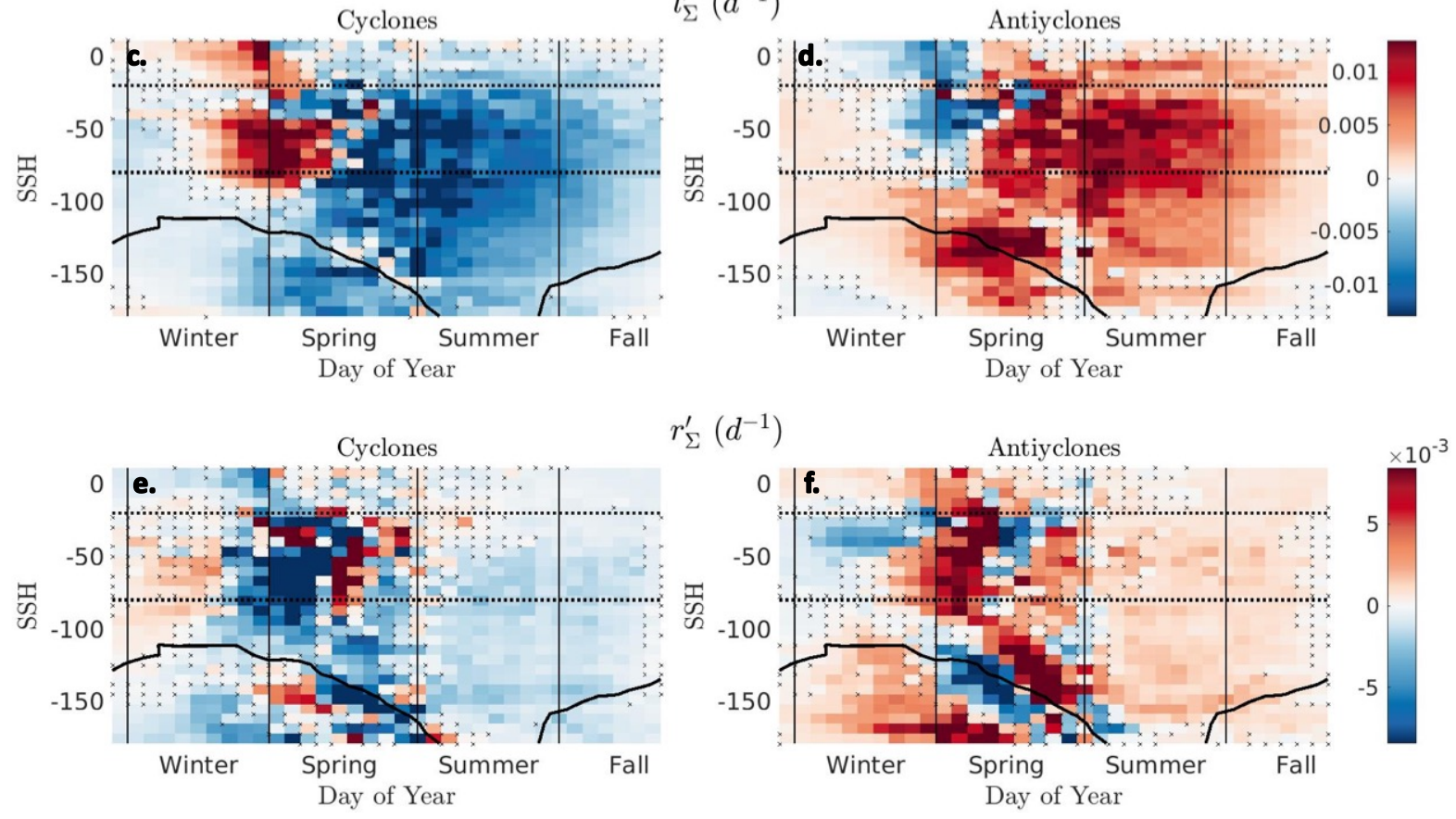

Figure 4-2: Hövmoller distributions of anomalous biological rate terms in Pacific eddies. Anomalous $(\mathbf{a}, \mathbf{b})$ division rates $\left(\mu_{\Sigma}^{\prime}\right),(\mathbf{c}, \mathbf{d})$ loss rates $\left(l_{\Sigma}^{\prime}\right)$ and $(\mathbf{e}, \mathbf{f})$ net population growth rates $\left(r_{\Sigma}^{\prime}\right)$ in cyclones (on the left) and anticyclones (on the right) are plotted as a function of season and $S S H$. Only eddies in the pacific sector $\left(80 W^{\circ}<\right.$ Lon $\left.<180 W^{\circ}\right)$ of the Southern Ocean (Lat $<40 S^{\circ}$ ) are included. $S S H$ serves as dynamic proxy for latitude to help align meanders in the $A C C$ during zonal averaging. The climatological ice edge (Ice Fraction $>80 \%$ ) is overlaid with a solid black contour. The ACC is denoted with dashed horizontal lines. Bins not statistically significant at the $95 \%$ confidence level are denoted with a black $\mathbf{x}$. 

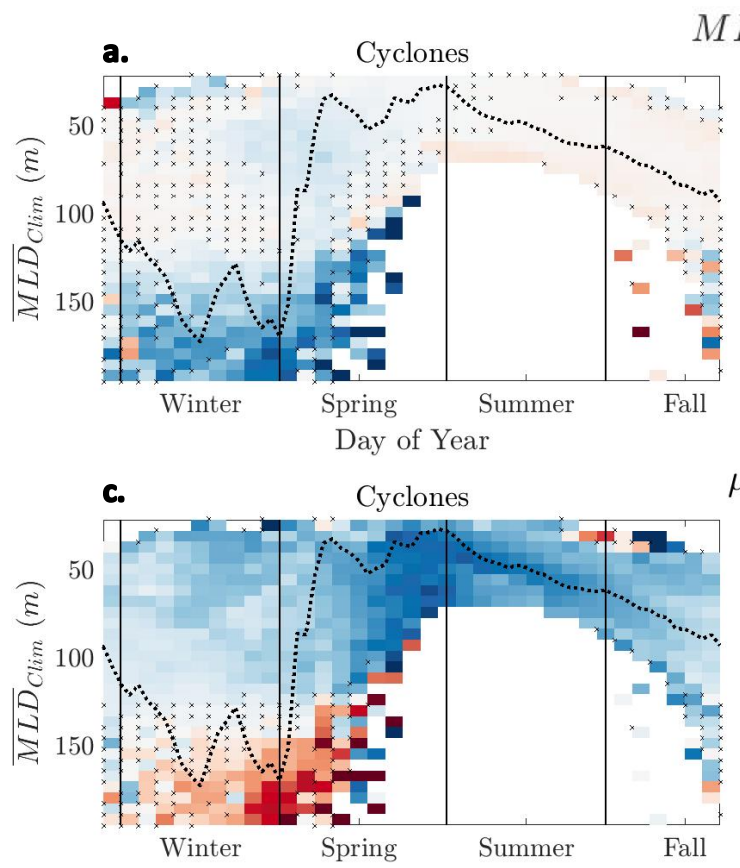

$\mu_{\Sigma}^{\prime}\left(d^{-1}\right)$ d.

b. Antiyclones
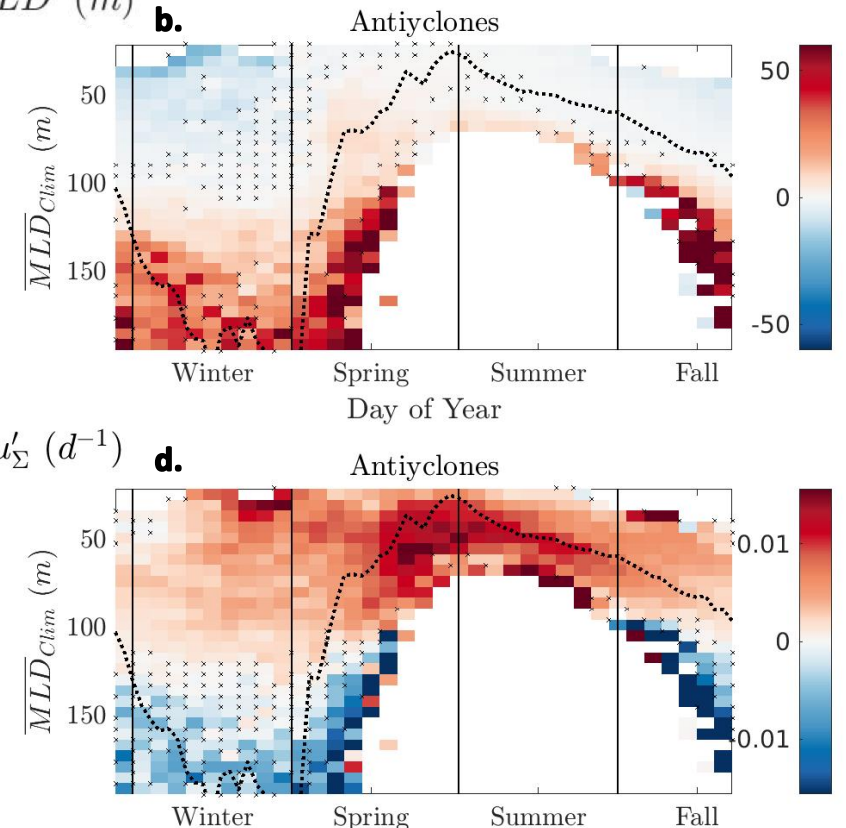

Day of Year

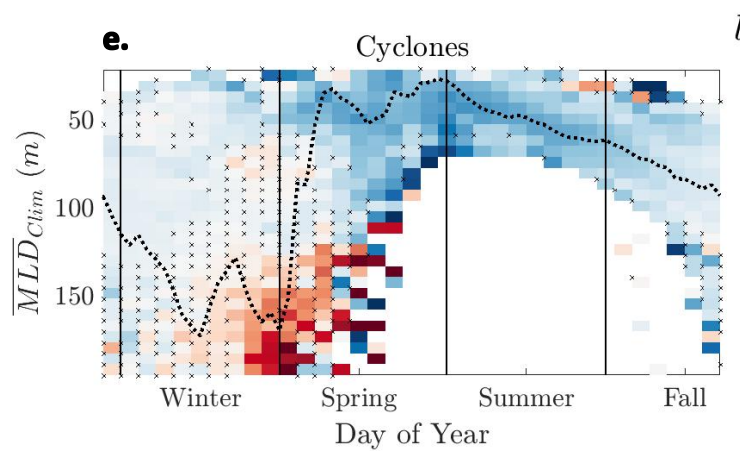

$l_{\Sigma}^{\prime}\left(d^{-1}\right) \mathbf{f}$.

Day of Year
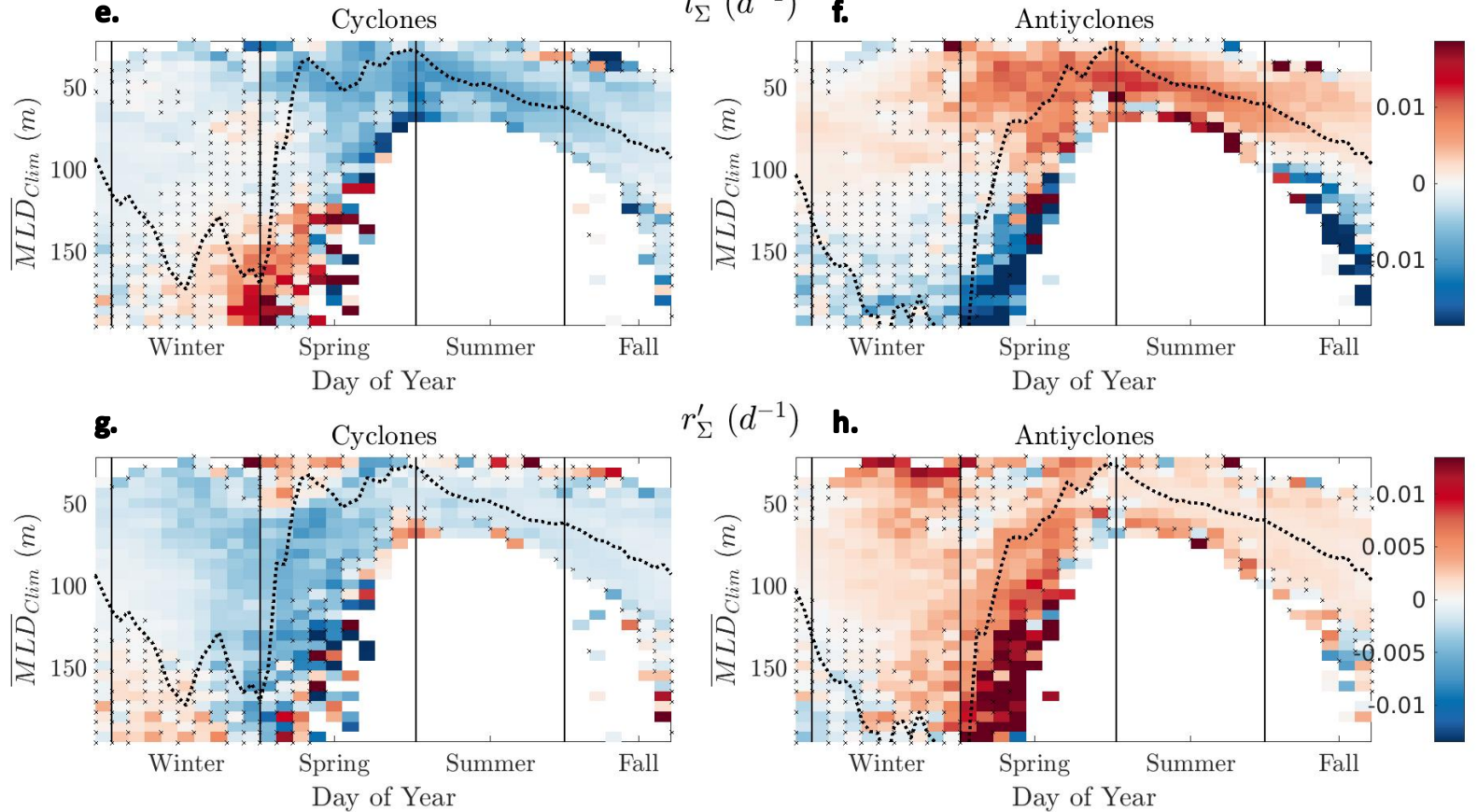

$r_{\Sigma}^{\prime}\left(d^{-1}\right)$ h. Antiyclones

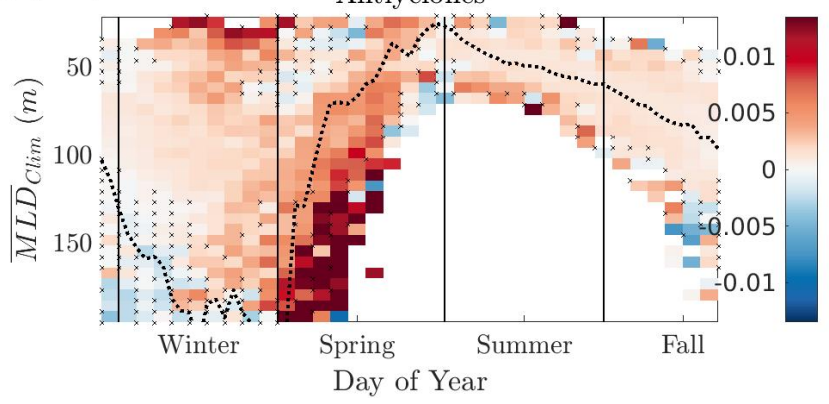

Figure 4-3: Variability in rate anomalies as a function of season and the background mixed layer depth $\left(\overline{M L D}_{C l i m}\right)$. Anomalous $(\mathbf{a}, \mathbf{b})$ mixed layer depths $\left(M L D^{\prime}\right),(\mathbf{c}, \mathbf{d})$ division rates $\left(\mu_{\Sigma}^{\prime}\right),(\mathbf{e}, \mathbf{f})$ loss rates $\left(l_{\Sigma}^{\prime}\right)$ and $(\mathbf{g}, \mathbf{h})$ net population growth rates $\left(r_{\Sigma}^{\prime}\right)$ in cyclones (on the left) and anticyclones (on the right) are plotted as a function of season and the $\overline{M L D}_{\text {Clim }}$. The mean mixed layer depth from the subset of large eddies in the Pacific ACC described in detail in Fig. 4-9 is overlaid with a dashed black line for reference. Bins that are not statistically significant at the $95 \%$ confidence level are denoted with a black $\mathbf{x}$. 


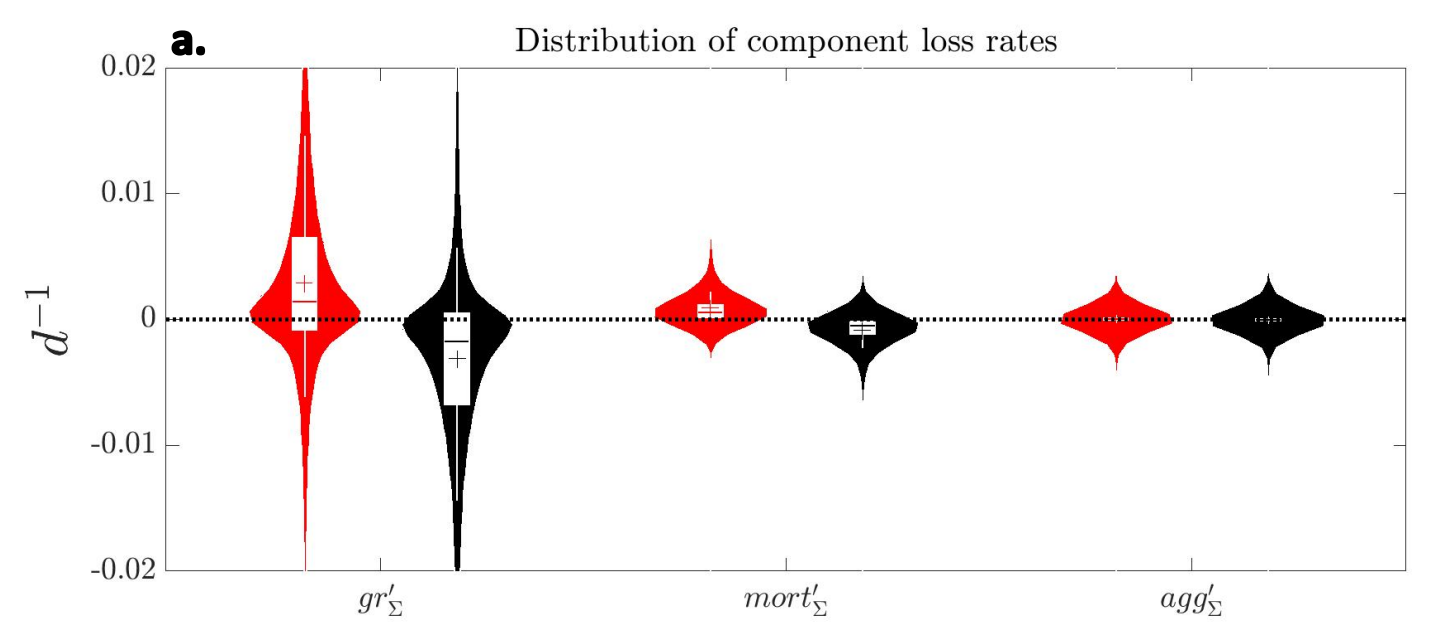

b.

Distribution of rate terms

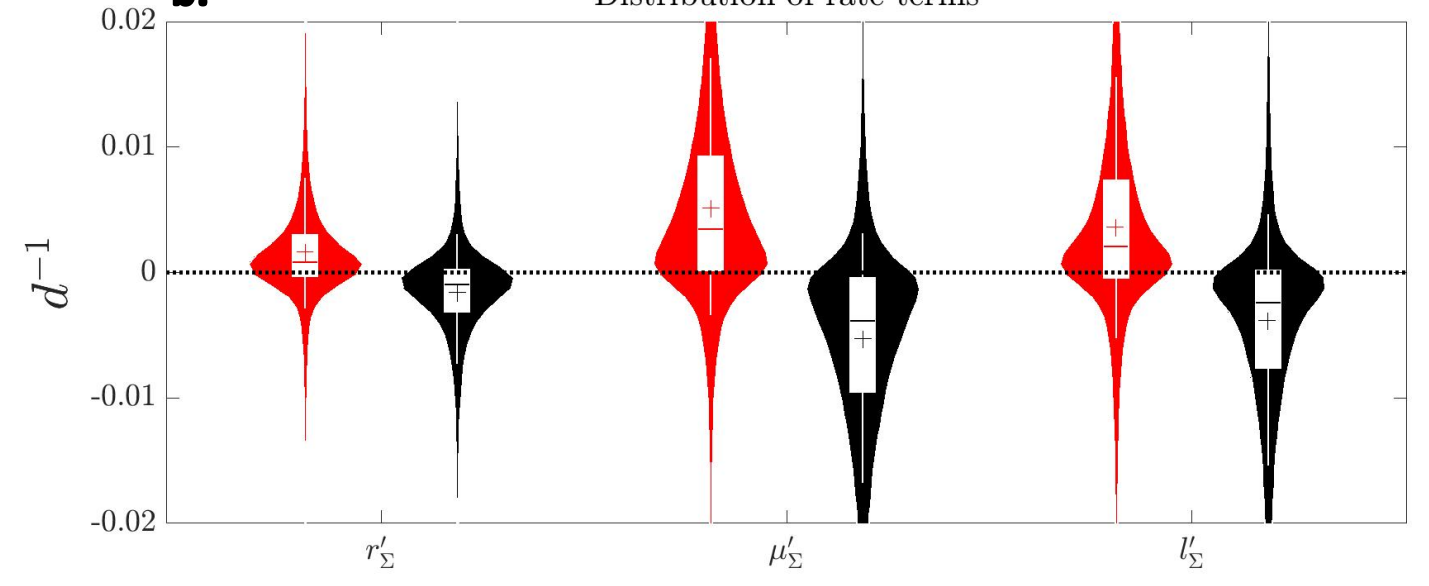

Figure 4-4: Distribution of anomalous rate terms. The distribution of (a) anomalous component loss rates and (b) all rate terms are plotted for all Southern Ocean cyclones (black) and anticyclones (red). The top panel only includes the component loss rate terms; grazing $\left(g r_{\Sigma}^{\prime}\right)$ is on the left, mortality $\left(\operatorname{mort}_{\Sigma}^{\prime}\right)$ is in the middle, and aggregation $\left(a g g_{\Sigma}^{\prime}\right)$ is on the right. Together these three anomalies comprise the total anomalous loss rate $\left(l_{\Sigma}^{\prime}\right)$ which is shown on the right of the bottom panel alongside anomalous division rates $\left(\mu_{\Sigma}^{\prime}\right)$ in the middle and anomalous net population growth rates $\left(r_{\Sigma}^{\prime}\right)$ on the left. In an given grid cell $r^{\prime}=\mu^{\prime}-l^{\prime}$ 


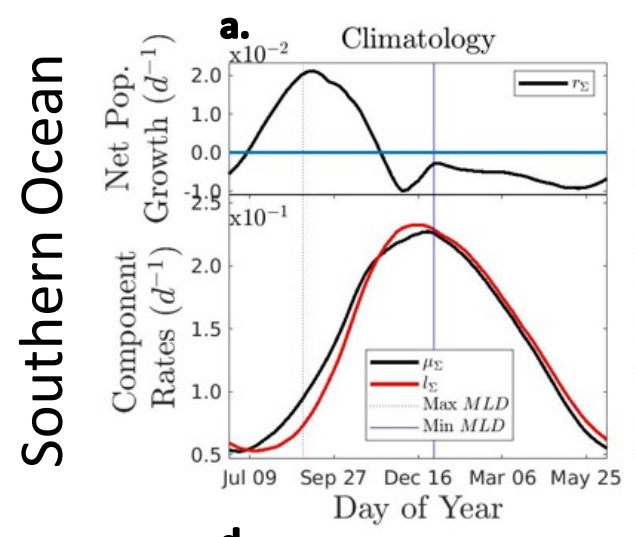

d.

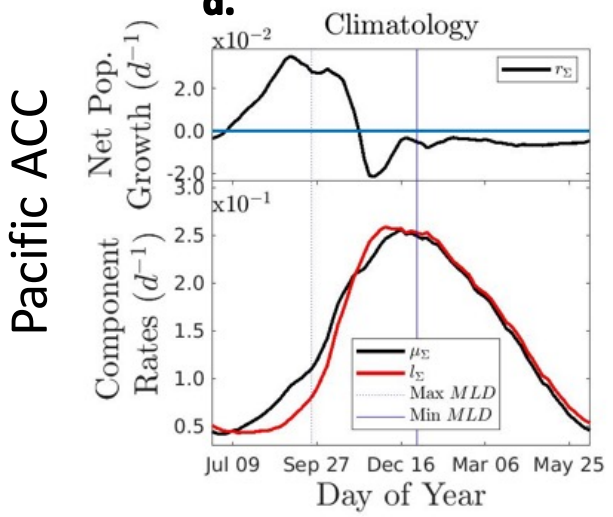

b.

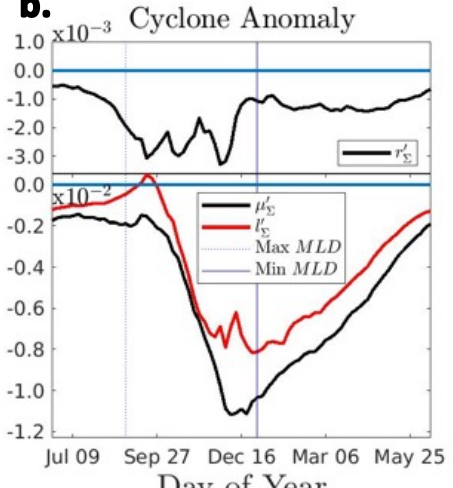

e.

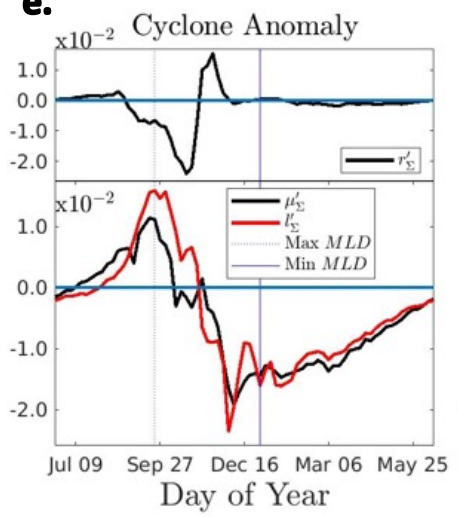

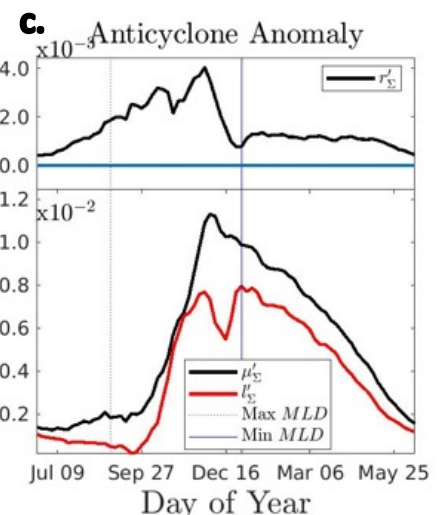

f.

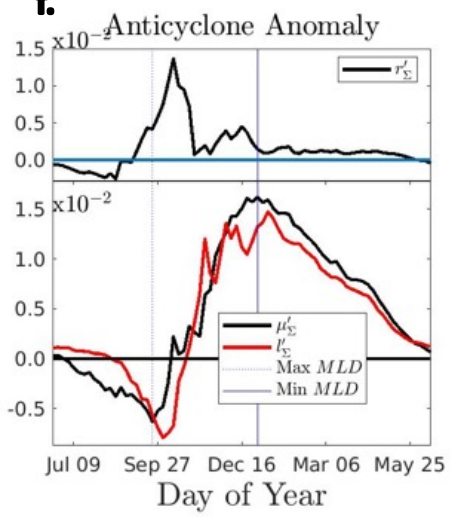

Figure 4-5: Seasonal evolution of rate terms. The seasonal evolution of $(\mathbf{a}, \mathbf{d})$ climatological and anomalous $(\mathbf{b}, \mathbf{c}, \mathbf{e}, \mathbf{f})$ population averaged rate terms is provided for $(\mathbf{a}-\mathbf{c})$ the average of all Southern Ocean eddies as well as (d-f) the subset of only large eddies in the deep mixing pacific sector of the ACC. (a, d) Climatologies, (b, e) cyclone anomalies, and $(\mathbf{c}, \mathbf{f})$ anticyclone anomalies are provided from left to right. Each panel is broken into the evolution of component rate terms $\left(\mu_{\Sigma}^{\prime}\right.$ in black; $l_{\Sigma}^{\prime}$ in red) on the bottom and the net population growth rate ( $r_{\Sigma}^{\prime}$ in black) on the top. The day of the climatologic maximum and minimum mixed layer depth is denoted with dashed and solid vertical blue lines, respectively. 

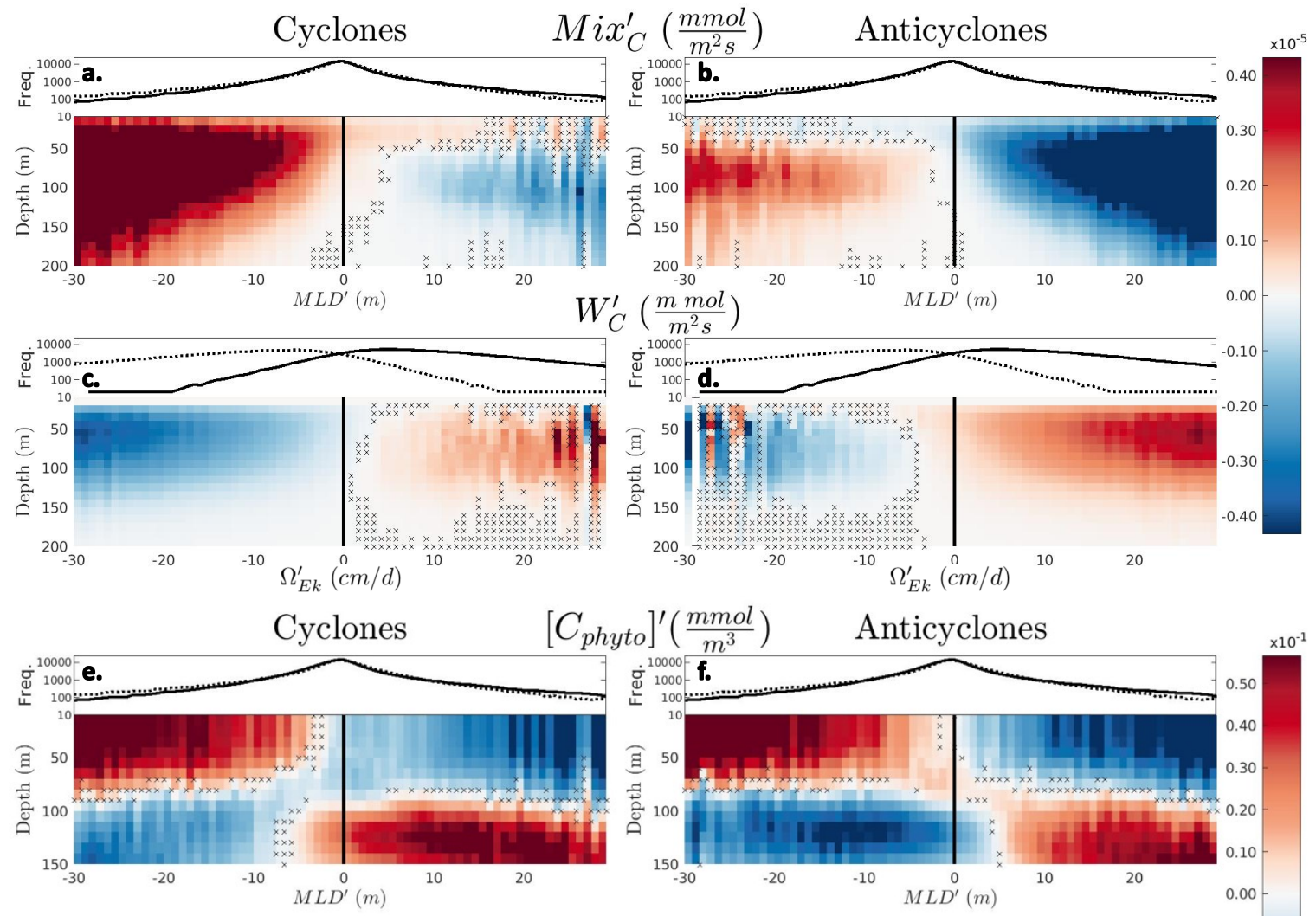

Anticyclones
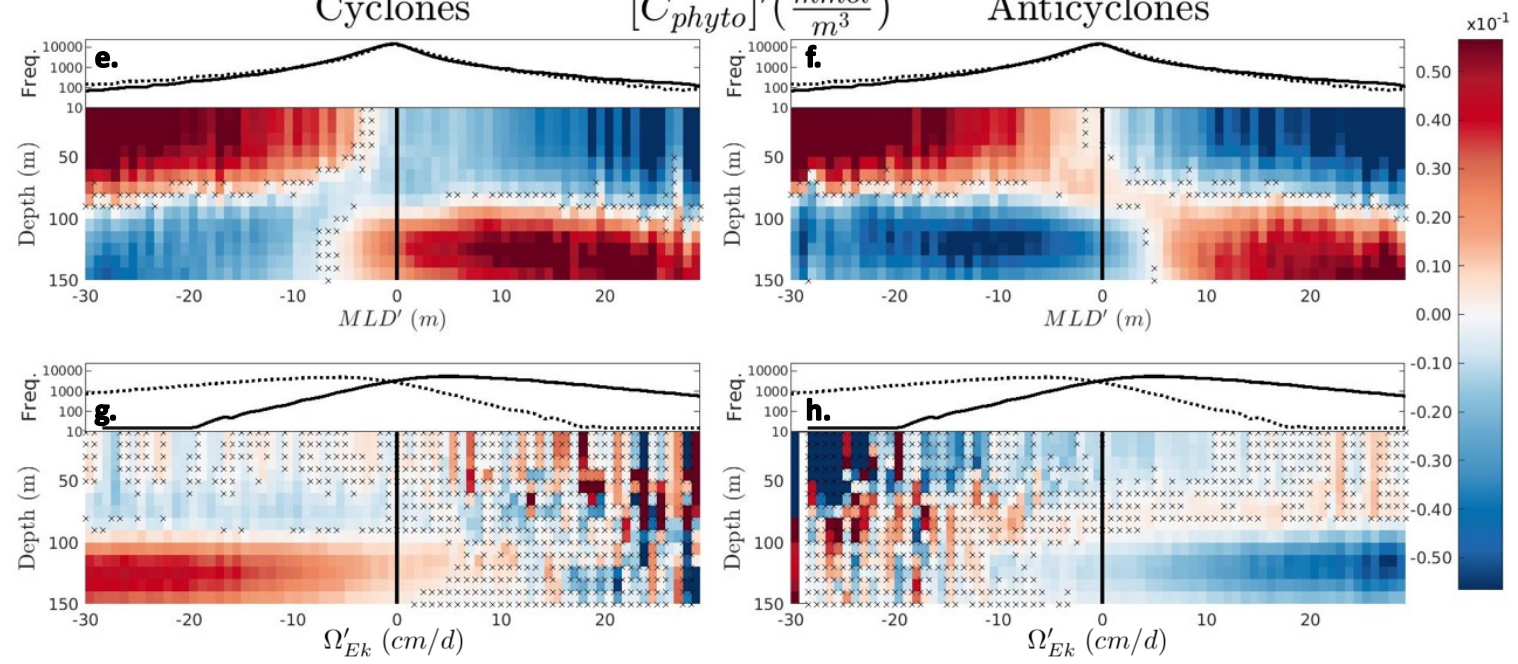

Figure 4-6: Anomalous biomass flux and concentration associated with vertical transport mechanisms. In the top half of the figure depth profiles are provided for the anomalous vertical (a, b) mixing flux of biomass $\left(M i x_{C}^{\prime}\right)$ and $(\mathbf{c}, \mathbf{d})$ advection flux of biomass $\left(W_{C}^{\prime}\right)$ averaged across all Southern Ocean cyclones (left) and anticyclones (right). $M i x_{C}^{\prime}$ and $W_{C}^{\prime}$ are plotted as a function of their driving mechanisms, the anomalous mixed layer depth $\left(M L D^{\prime}\right)$ and the anomalous eddy-induced Ekman pumping velocity $\left(\Omega_{E k}^{\prime}\right)$, respectively. Fluxes are positive in the upward direction. In the bottom half of the figure, (e-h) depth profiles are provided for the anomalous biomass concentration associated with the same vertical transport mechanisms. Bins with anomalies that are statistically insignificant from 0 at the $95 \%$ confidence level are marked with a black $\mathbf{x}$. The frequency of eddy realizations that fall in each bin on the $\mathrm{x}$-axis is plotted above the corresponding profiles. Cyclonic (solid lines) and anticyclonic (dashed lines) distributions are included in each plot to ease comparison. 

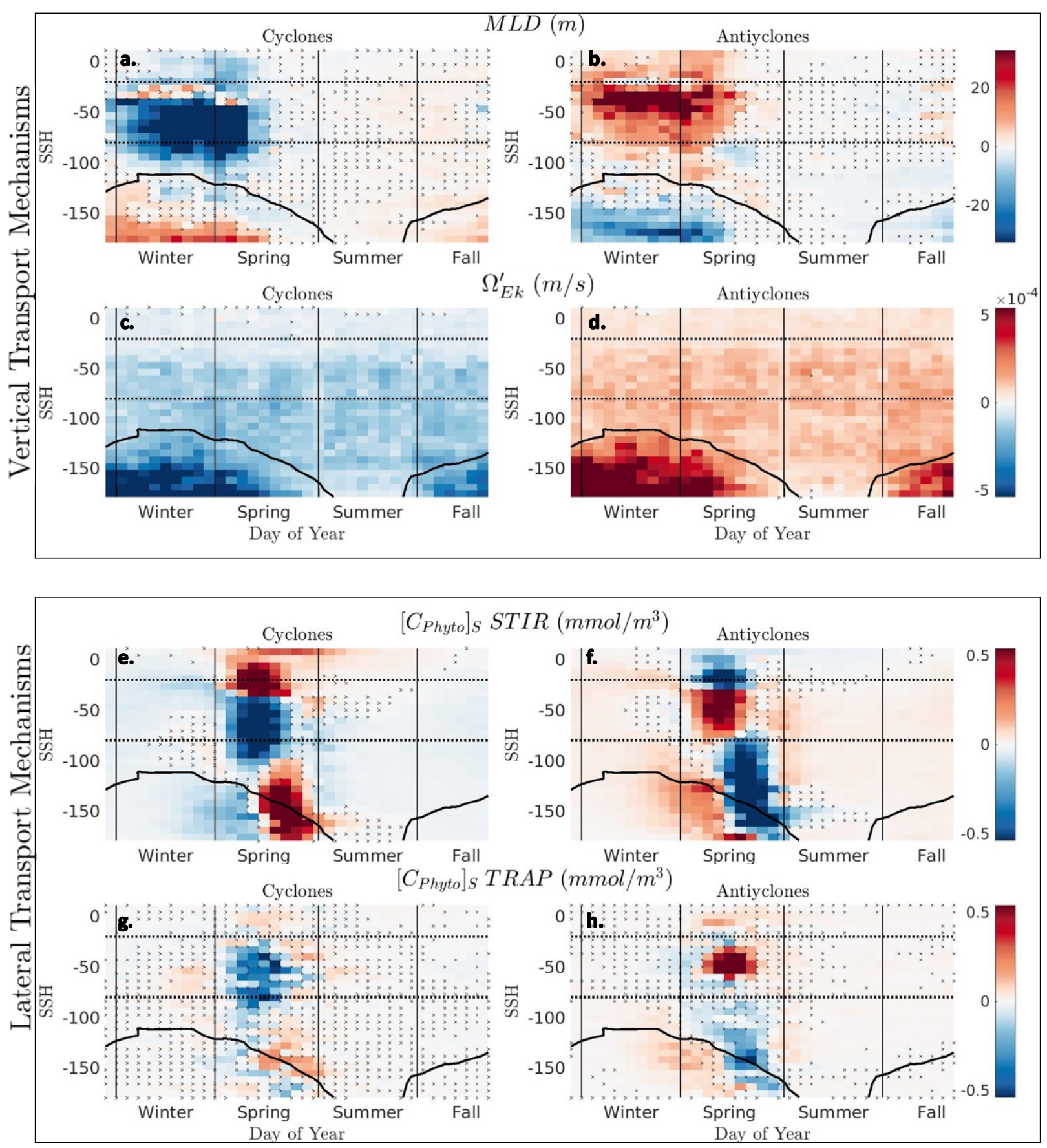

Figure 4-7: Hövmoller distributions of anomalous physical transport mechanisms in Pacific eddies. Identical to Fig. 4-2 but for vertical (top four panels) and lateral (bottom four panels) physical transport mechanisms. Anomalous $(\mathbf{a}, \mathbf{b})$ mixed layer depths $\left(M L D^{\prime}\right)$ and $(\mathbf{c}, \mathbf{d})$ eddy-induced Ekman pumping velocities $\left(\Omega_{E k}^{\prime}\right)$ to characterize the vertical transport mechanisms. The potential surface biomass anomalies that could arise from $(\mathbf{e}, \mathbf{f})$ stirring $(S T I R)$, and $(\mathbf{g}, \mathbf{h})$ trapping $(T R A P)$ characterize the lateral transport mechanisms. 

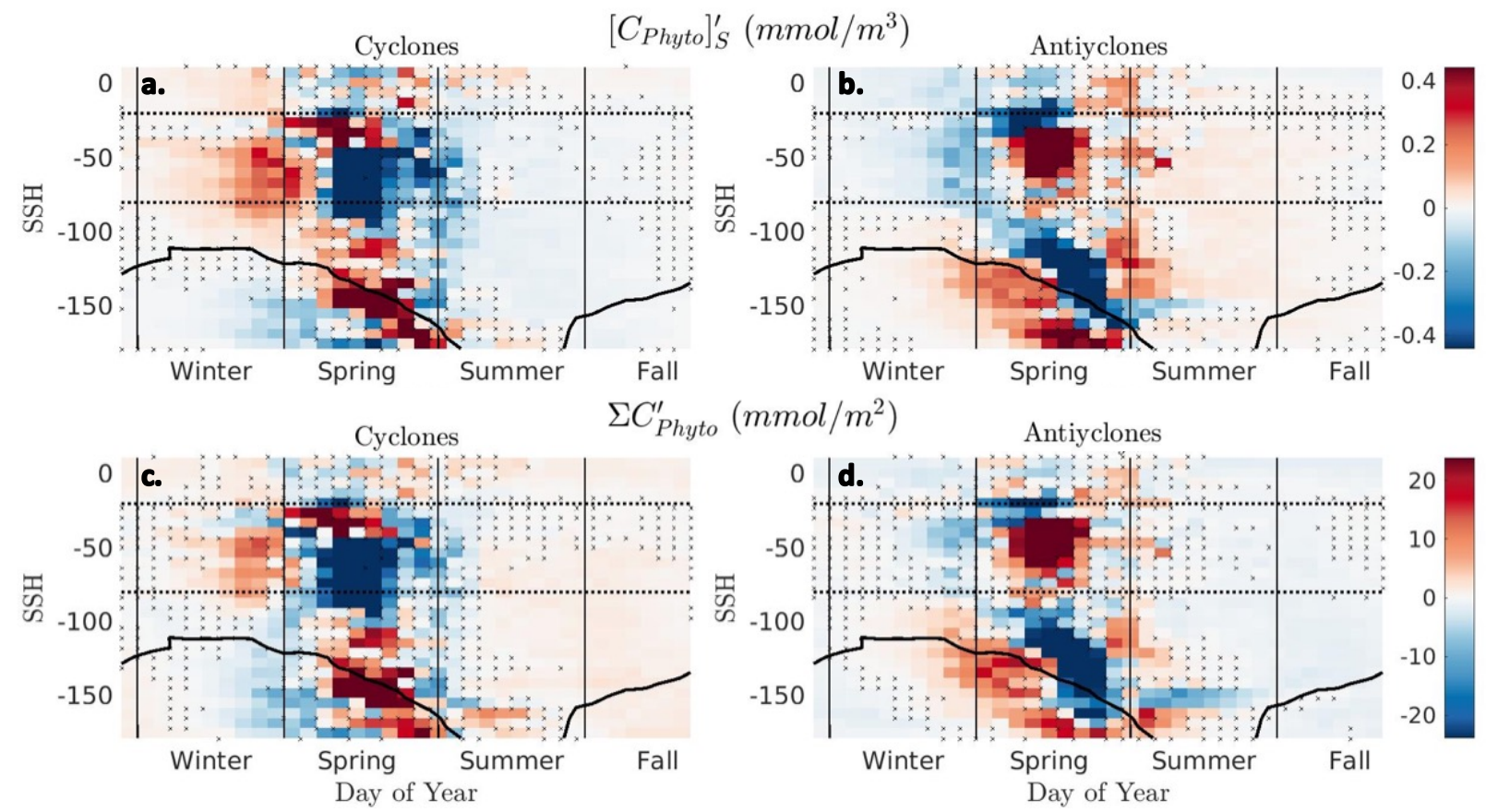

Figure 4-8: Hövmoller distributions of anomalous biomass in Pacific eddies. Identical to Fig. 4-2 but for anomalous $(\mathbf{a}, \mathbf{b})$ surface biomass concentrations $\left(\left[C_{P h y t o}\right]_{S}^{\prime}\right)$, and $(\mathbf{c}, \mathbf{d})$ depth integrated biomass inventories $\left(\Sigma C_{\text {Phyto }}^{\prime}\right)$. 

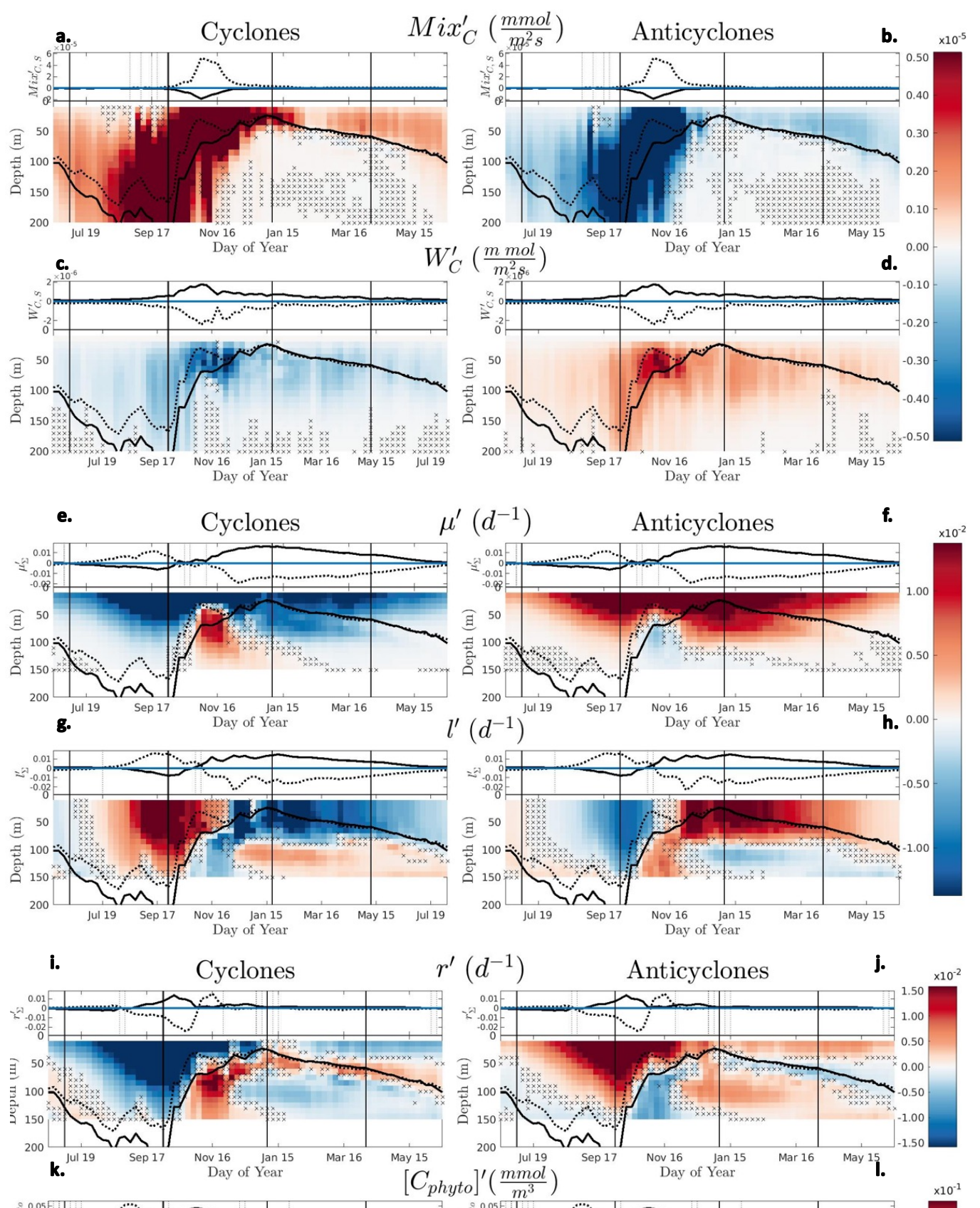

$r^{\prime}\left(d^{-1}\right)$

Anticyclones

j.
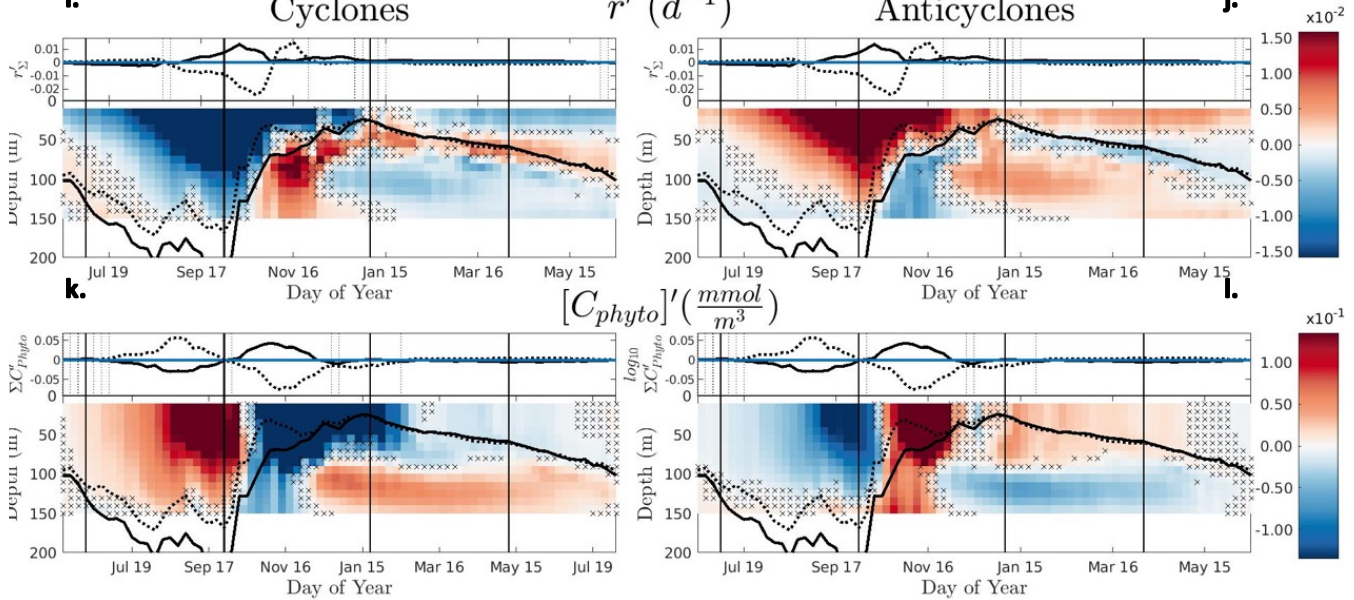

Figure 4-9: Seasonal variability of depth resolved eddy anomalies in the deep mixing Pacific ACC. Qualifying eddy realizations (see 4.2.6) are sorted into daily bins and averaged. Profiles of seasonal variability in $M i x_{C}^{\prime}, W_{C}^{\prime}, \mu_{\Sigma}^{\prime}, l_{\Sigma}^{\prime}, r_{\Sigma}^{\prime}$, and $\left[C_{\text {phyto }}\right]^{\prime}$ are plotted for cyclones on the left, and anticyclone on the right. The mean $M L D$ in cyclones (dashed) and anticyclones (solid) is overlaid in black. Bins with anomalies that are statistically insignificant from 0 at the $95 \%$ confidence level are marked with a black x. Above each profile plot is a plot of either the corresponding (a-d) carbon flux into the surface ocean or (e-i) the depth-averaged population mean. Bins in which the difference between cyclone and anticyclone anomalies are statistically insignificant at the $95 \%$ confidence level are marked with a dashed vertical line. Solid vertical lines delineate seasons. 

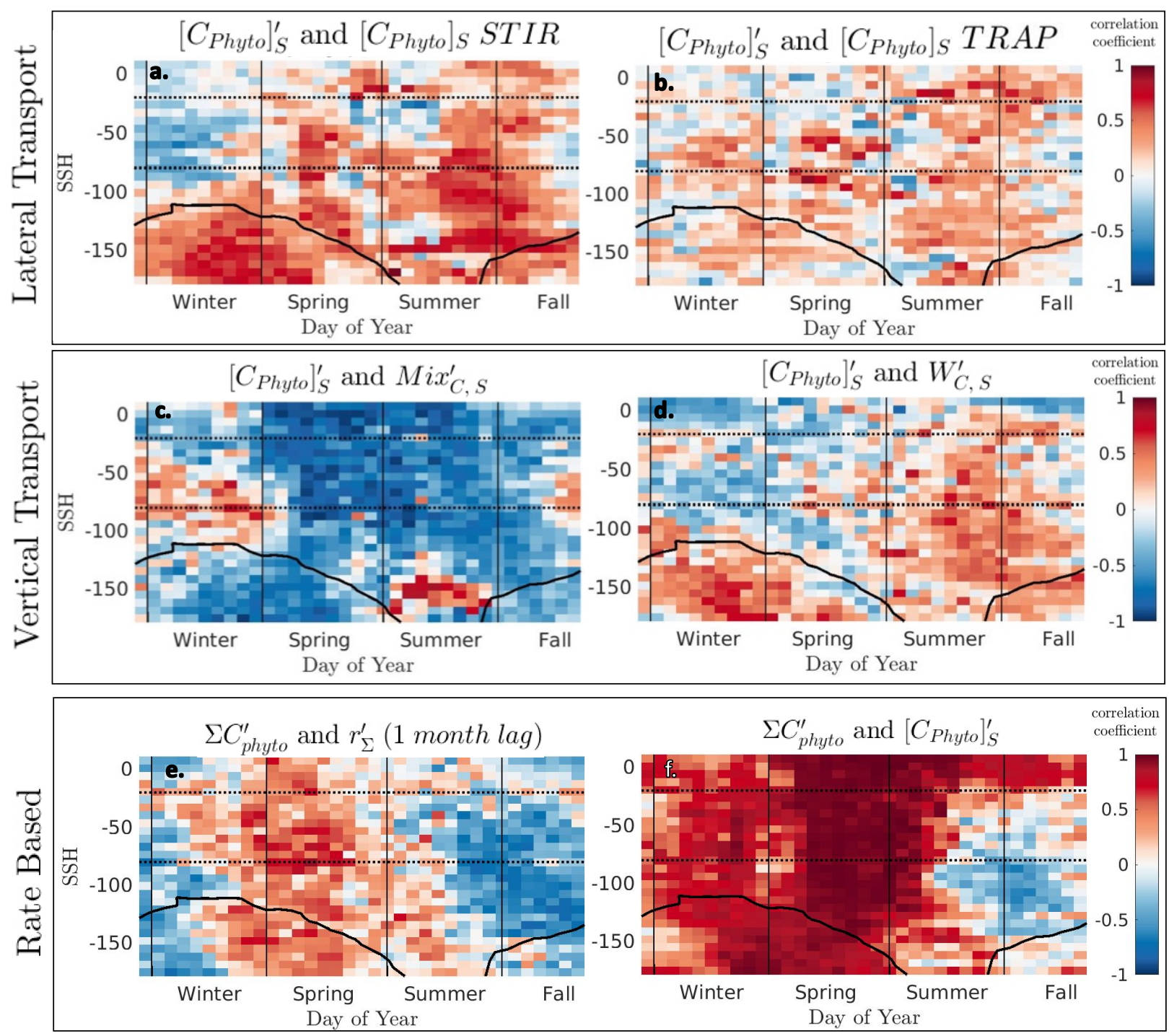

Figure 4-10: Hövmoller distributions of the correlation between anomalous biomass and various potential mechanisms. In the top row, the potential for lateral stirring to induce anomalous biomass is correlated with the actual simulated biomass anomaly for both (a) surface concentrations and (b) the depth integrated inventory. In the middle row the anomalous flux of carbon into the surface via (c) diabatic mixing $\left.\left(M i x_{C, S}^{\prime}\right)\right)$ and $(\mathbf{d})$ advection $\left.\left(W_{C, S}^{\prime}\right)\right)$ is correlated with actual simulated anomalous surface concentration $\left(\left[C_{P h y t o}\right]_{S}^{\prime}\right)$. In the bottom row the anomalous biomass inventory $\left(\Sigma C_{P h y t o}^{\prime}\right)$ is correlated with the anomalous population averaged net population growth rates which have been lagged by one month $\left(r_{\Sigma}^{\prime}\right.$ (lagged $\left.)\right)$ as well as the anomalous surface concentrations $\left(\left[C_{P h y t o}\right]_{S}^{\prime}\right)$. Type II correlations are computed independently in each spatial/temporal bin and include both cyclones and anticyclones. Only the Pacific basin is included for direct comparison to Fig. 4-2, 4-7, and 4-8. The climatologic ice contour is overlaid in black. 


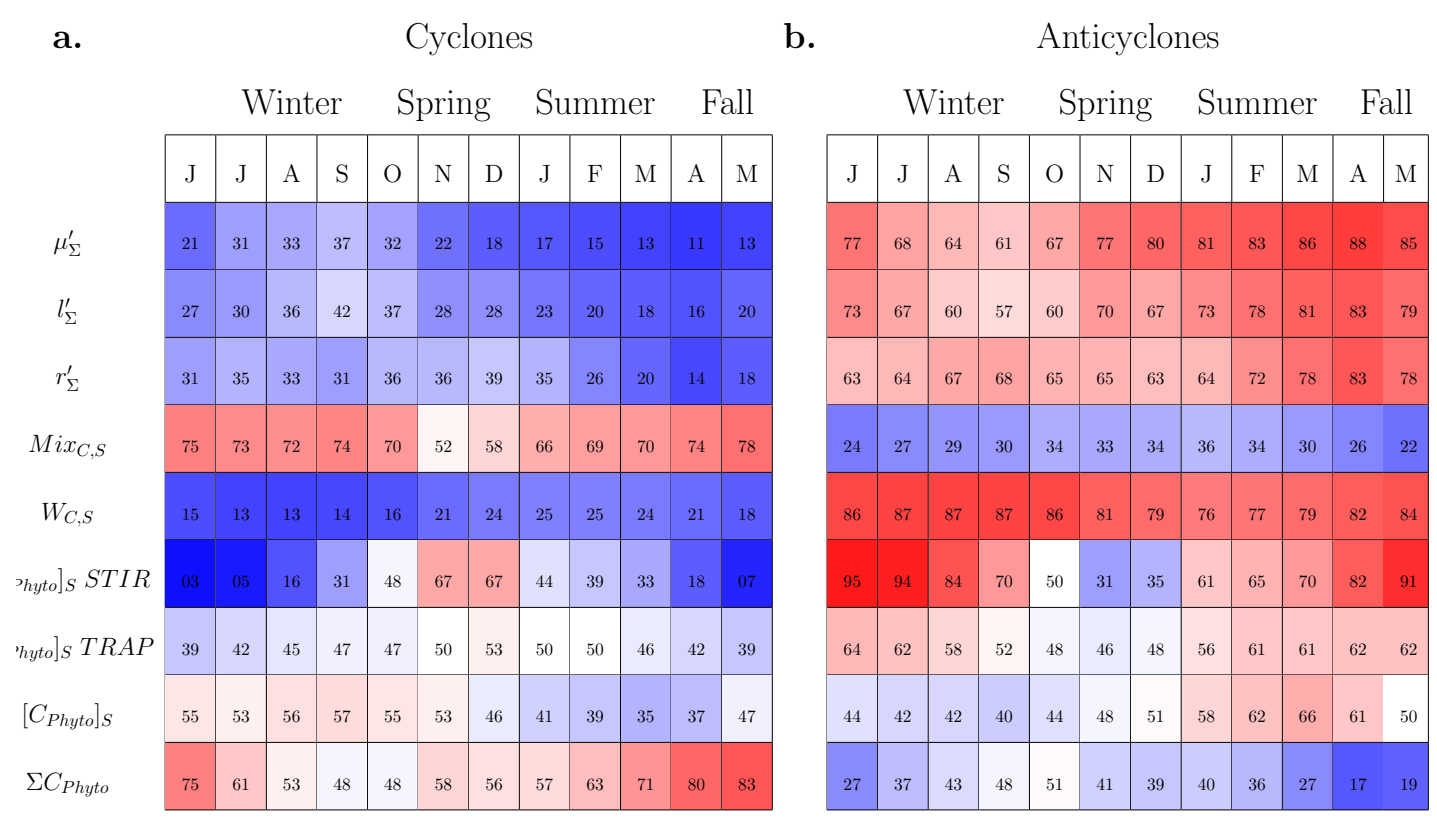

Table 4.1: Seasonal distribution of frequency of eddies with positive anomalies. The frequency (\%) of all Southern Ocean (a.) cyclones and (b.) anticyclones with a positive anomaly in a given climatologic month is plotted for various variables.

a. Southern Ocean

\begin{tabular}{|c|c|c|c|c|}
\hline$\mu_{\Sigma}^{\prime}$ & $l_{\Sigma}^{\prime}$ & $g r_{\Sigma}^{\prime}$ & $\operatorname{mort}_{\Sigma}^{\prime}$ & $a g g_{\Sigma}^{\prime}$ \\
\hline 1.0 & .73 & .69 & . 40 & -.10 \\
\hline .73 & 1.0 & .98 & .30 & -.09 \\
\hline .22 & -.05 & -.05 & .23 & -.37 \\
\hline
\end{tabular}

b.

$\mathrm{ACC}$

\begin{tabular}{|c|c|c|c|c|}
\hline$\mu_{\Sigma}$ & $l_{\Sigma}^{\prime}$ & $g r_{\Sigma}^{\prime}$ & $\operatorname{mort}_{\Sigma}^{\prime}$ & $a g g_{\Sigma}^{\prime}$ \\
\hline 1.0 & .77 & .75 & .22 & -.08 \\
\hline .77 & 1.0 & .98 & .18 & .10 \\
\hline .06 & -.21 & -.23 & .31 & -.24 \\
\hline
\end{tabular}

Table 4.2: Correlation between rate terms. The correlation between various rate term anomalies is plotted for all (a.) all Southern Ocean eddies and (b.) strictly ACC eddies. Correlations are taken between all eddy realizations at all times within the specified geographic domain. 


\begin{tabular}{|c|c|c|c|c|c|c|c|c|c|c|c|}
\multicolumn{4}{c}{ Winter } & \multicolumn{1}{c}{ Spring } & Summer & Fall \\
\hline $\mathrm{J}$ & $\mathrm{J}$ & $\mathrm{A}$ & $\mathrm{S}$ & $\mathrm{O}$ & $\mathrm{N}$ & $\mathrm{D}$ & $\mathrm{J}$ & $\mathrm{F}$ & $\mathrm{M}$ & $\mathrm{A}$ & $\mathrm{M}$ \\
\hline
\end{tabular}

\begin{tabular}{|c|c|c|c|c|c|c|c|c|c|c|c|}
\hline \multicolumn{12}{|c|}{ Cyclones } \\
\hline $3069(1.7)$ & $1816(0.8)$ & $1712(0.3)$ & $-2251(-0.9)$ & & $-4335 \quad(-0.2)$ & $764(0.3)$ & $1381(0.2)$ & $4949(1.2)$ & 3614 & 4178 & $4166(1.9)$ \\
\hline \multicolumn{4}{|c|}{$425(0.1)$} & \multicolumn{3}{|c|}{$-4223(-0.5)$} & \multicolumn{3}{|c|}{$3241(0.9)$} & \multicolumn{2}{|c|}{$3804(1.7)$} \\
\hline \multicolumn{12}{|c|}{$743(0.5)$} \\
\hline \multicolumn{12}{|c|}{ Antiyclones } \\
\hline$-2599(-1.3)$ & $-2273(-1.1)$ & $-1914(-0.5)$ & $2033(0.8)$ & $10521(1.9)$ & $-6051(-0.3)$ & $-3609(-0.2)$ & $1008(-0.1)$ & $-4737(-0.9)$ & $-5187(-1.2)$ & $-4598 \quad(-1.5)$ & $-3397(-1.5)$ \\
\hline & & $-717(-0.2)$ & & & $82(0.4)$ & & & $-2997(-0.8)$ & & -3531 & $(-1.4)$ \\
\hline \multicolumn{12}{|c|}{$-1765(-0.5)$} \\
\hline \multicolumn{12}{|c|}{ All Eddies } \\
\hline $470(0.3)$ & $-458(-0.3)$ & $-202(-0.2)$ & $-218(-0.1)$ & $-594(0.1)$ & $(-1,0.9)$ & $-2845(0.0)$ & $2389(0.2)$ & $213(0.3)$ & $-1573(-0.0)$ & $-420(0.0)$ & $768(0.4)$ \\
\hline \multicolumn{4}{|c|}{$-292(-0.2)$} & \multicolumn{3}{|c|}{$-4140(-0.2)$} & \multicolumn{3}{|c|}{$243(0.1)$} & \multicolumn{2}{|c|}{$273(0.3)$} \\
\hline & & & & & -1022 & $(0.03)$ & & & & & \\
\hline
\end{tabular}

b.

Integrated Across ACC

\begin{tabular}{|c|c|c|c|c|c|c|c|c|c|c|c|}
\hline \multicolumn{12}{|c|}{ Cyclones } \\
\hline $820(1.2)$ & $650(1.0)$ & $751(1.4)$ & $-1518 \quad(-0.7)$ & $-7540(-3.9)$ & $12655(-1,1$ & $-4069(-1.5)$ & $1018(1.0)$ & $4045(3.3)$ & $2318(2.4)$ & $1546(1.9)$ & $1193(1.7)$ \\
\hline & \multicolumn{3}{|c|}{$-39(0.6)$} & \multicolumn{3}{|c|}{$-7876(-3.0)$} & \multicolumn{3}{|c|}{$2364(2.2)$} & \multicolumn{2}{|c|}{$1187(1.6)$} \\
\hline \multicolumn{12}{|c|}{$-1184(0.3)$} \\
\hline \multicolumn{12}{|c|}{ Antiyclones } \\
\hline \multirow[t]{2}{*}{$-839(-1.4)$} & $-882(-1.5)$ & $-1477(-2.2)$ & $-2030(-2.1)$ & $2778(1.2)$ & $9144(3.8)$ & $1257(0.4)$ & $152(0.1)$ & $-2558(-1.7)$ & $-894(-1.0)$ & $-857(-1.4)$ & $-986(-1.6)$ \\
\hline & & $-1463(-2.0)$ & & \multicolumn{3}{|c|}{$4228(1.7)$} & & \multicolumn{2}{|l|}{$-1007(-0.8)$} & \multicolumn{2}{|c|}{$270(-1.5)$} \\
\hline \multicolumn{12}{|c|}{$1099(-0.6)$} \\
\hline \multicolumn{12}{|c|}{ All Eddies } \\
\hline$-19(-0.2)$ & $-232(-0.5)$ & $-725(-0.8)$ & $-3548 \quad(-2.8)$ & $-4760(-2.8)$ & $-3510(-0.2)$ & $-2812(-1.0)$ & $1170(1.1)$ & $1487(1.6)$ & $1424(1.5)$ & $689(0.5)$ & $208(0.1)$ \\
\hline \multicolumn{3}{|c|}{$-1502(-1.4)$} & \multicolumn{3}{|c|}{$-3648(-1.3)$} & \multicolumn{3}{|c|}{$1357(1.4)$} & \multicolumn{2}{|c|}{$293(0.2)$} & \\
\hline & & & & & -913 & & & & & & \\
\hline
\end{tabular}

Table 4.3: Spatially integrated anomalous biomass. The total, depth integrated anomalous carbon biomass in each eddy is spatially integrated across cyclones, anticyclones, and all eddies within the (a.) the entire Southern Ocean and (b.) ACC. Spatial integration is sensitive to both the number and size of eddies in a given region. Values are expressed for an average day in a given climatologic month, season, or year. To get a better sense of the scale of these numbers relative to how much biomass is in eddies, the percentage of anomalous biomass relative to the spatially integrated climatologic biomass in the corresponding set of eddies is included in parentheses. 
Chapter 5

Southern Ocean Iron Fertilization: An ARGUMENT AGAINST COMMERCIALIZATION BUT FOR CONTINUED RESEARCH AMIDST LINGERING UNCERTAINTY. 


\begin{abstract}
In light of the challenges impeding substantive global action on climate change mitigation, some have begun to look at geoengineering as a viable alternative. Ocean Iron Fertilization (OIF) is one such strategy that seeks to increase oceanic drawdown of CO2 by stimulating marine phytoplankton growth in large iron-limited swaths of the Southern Ocean. Unfortunately, there are serious doubts that a global scale, iron-induced sequestration pathway could be sustainable, meaningful, additional, permanent, free of leakage, and absent of adverse side effects. While reduced uncertainty could one day reveal a reasonable, measured, approach to leverage OIF under unilateral authority and dynamic management, commercialization is, and will remain, unviable. Measurement challenges, unreliable auditing, ambiguous baselines compromised by high frequency variability, poor enforcement, and severe externalities, would together cripple a market-based approach to implementation and should preclude adoption into emerging compliance offset markets. Unfortunately, commercial ventures on voluntary offset markets motivated by the relative ease of OIF, market incentives and a loose regulatory framework have and may continue to emerge contrary to the scientific consensus. Over time, further research may or may not validate OIF as a justifiable risk, it will, however, almost certainly help delegitimize the reckless commercialization of OIF and dismiss the notion that the market can constrain such a complex and non-linear system without serious consequences.
\end{abstract}

\title{
5.1 Introduction/Background
}

Although geophysical scientists can say with increasing certainty that anthropogenic climate change poses a serious threat to human life and livelihood [IPPC AR5 2014], decisive political efforts to reduce emissions have proven slow and insufficient [Clark 2012; Hovi et al. 2009; Shear 2018]. Hedging against the challenges of a collective action crisis of this scale [Thompson 2006] many have turned to adaptation to confront a future potentially plagued by unchecked fossil fuel consumption. The most dramatic of these adaptation strategies, known broadly as geoengineering, can be categorized as any deliberate, large-scale, manipulation of natural processes to effect the climate system, ostensibly to curb the effects of global warming.

Geoengineering is an understandably enticing proposition. By targeting the symptoms of climate change for pennies on the dollar, geoengineering evades the daunting economic and cultural sacrifices that serious mitigation might demand. While its proponents preach the need to leverage human ingenuity [Lynas 2011], the temptation of such a convenient deus ex machina raises some serious red flags. Critics stress that in the complex, non-linear earth system the treatment may not work as advertised, allowing the underlying cause to aggravate while unpredictable, side effects threaten to fester [Lynas 2011; Robock et al. 2008]. Despite the 
controversy, the promise of a silver-bullet techno-fix has proven difficult to ignore, attracting attention from activists [Keith 2013], policy makers [Full Committee Hearing - Geoengineering, Part I 2009; Reichle et al. 1999], and scientists alike [Yoon et al. 2016].

One of the most prominent geoengineering strategies is carbon sequestration. The global carbon cycle, which influences climate via the radiative capacity of atmospheric $\mathrm{CO}_{2}$, largely operates on two time scales. The 'slow' carbon cycle, driven by tectonic activity, is balanced by volcanic outgassing and geological weathering [Berner 1990]. The 'fast' carbon cycle, driven by biology, is balanced by the reduction of inorganic carbon by photosynthesis and the oxidation of organic carbon by respiration [Riebeek 2011]. Although these two cycles are naturally linked by the eventual fossilization of organic matter, the rapid anthropogenic consumption of fossil fuels is pumping $\mathrm{CO} 2$ out of the 'slow' cycle much faster than natural cycles can compensate for [Chisholm et al. 2001]. The goal of carbon sequestration then is to geoengineer a carbon sink that is capable of routing carbon back into the 'slow' carbon cycle at a rate consummate with which we are extracting it.

Ocean Iron Fertilization (OIF) is one of several carbon sequestration strategies (see Fig. 5-1). OIF seeks to harness the power of ocean biogeochemistry to amplify atmospheric $\mathrm{CO}_{2}$ drawdown by augmenting inefficiencies in phytoplankton productivity. Microscopic marine phytoplankton account for roughly half of the photosynthetic carbon fixation on earth [Falkowski et al. 2000] and thanks to the rapid turnover time of their population $(\sim 1$ week $)$ they are able to influence climate on a much faster timescale [Falkowski 2002] than terrestrial plants. Although most of the carbon fixed by marine photosynthesis is rapidly recycled and released back to the atmosphere, a small fraction $(\sim 15 \%)$ sinks deep into the ocean where it might remain sequestered for tens to hundreds of years [Laws et al. 2000]; This process is known as the biological pump [de la Rocha 2006]. OIF hopes to increase the strength and efficiency of the biological pump thereby stimulating greater net atmospheric $\mathrm{CO}_{2}$ drawdown into the ocean.

Variability in the biological pump is widely accepted as an important factor in regulating glacial-interglacial cycles in atmospheric $\mathrm{CO}_{2}$ [Berner 1991; Sigman and Boyle 2000], however the precise mechanisms that drive changes to the biological pump are less clear [Falkowski 1997]. In the late 1980s John Martin proposed that iron was limiting primary production over large swaths of the ocean [Martin and Fitzwater 1988]. Iron is a micronutrient essential to phytoplankton growth but only required in small concentrations relative to macronutrients like Nitrogen and Phosphorus. Regions, known as HNLCs (High Nitrate, Low Chlorophyll), that yield low primary productivity despite an abundance of unutilized macronutrients are now thought to iron-limited. Martin went on to hypothesize that variability in the airborne deposition of iron over HNLC regions has triggered variability in primary productivity, the biological pump, and the net drawdown of atmospheric $\mathrm{CO}_{2}$ over glacial-interglacial cycles 135 
[Martin 1990]. Following the Martin Hypothesis, OIF seeks to fertilize HNLCs with the deliberate addition of iron in order to increase the strength of the biological pump and enhance oceanic drawdown of atmospheric $\mathrm{CO}_{2}$.

Research over the ensuing decades ranging from shipboard in-vitro incubations [Martin et al. 1991] to mesoscale in-situ fertilization experiments [Yoon et al. 2016] has largely confirmed that iron fertilization can in fact stimulate primary production in HNLC regions. It is decidedly less clear, however, whether or not that increased productivity is routed into even localized export[Boyd et al. 2007]. When considered at a global scale, there remains a great deal of doubt over the validity of a sustainable iron induced sequestration pathway [Winckler et al. 2016]. Additional concerns over the practical challenges of creating a credible auditing framework and the potential for unpredictable, adverse side effects create further complications.

Nevertheless, the lure of OIF remains theoretically seductive. HNLCs cover roughly a third of the world's oceans [Boyd et al. 2007] and if continuous fertilization was generously assumed to utilize and export all previously unutilized nutrients, early models predicted an atmospheric drawdown as high as 50-100 ppm $\mathrm{CO}_{2}$ [Aumont and Bopp 2006]. Despite problematical assumptions clouding these model estimates, geochemical proxies in ice cores [Winckler et al. 2008] linking elevated iron to depressed atmospheric CO2 and recent observations of localized export after an artificially induced bloom [Smetacek et al. 2012] have provided hope for proponents of OIF. If optimistic estimates for sequestration were even approached, OIF could potentially account for one of Pacala and Socolow's twelve proposed stabilization wedges [Pacala and Socolow 2004], enough to garner considerable attention [Buesseler et al. 2008a].

Amidst lingering uncertainty clouding the implementation, risk, and feasibility of OIF two predominant questions emerge. First, given the scope of the climate crisis could the commercialization of OIF under emerging compliance offset markets be a valuable piece of the solution? Second, is it prudent to dedicate valuable scientific resources to continuing incremental research targeted at reducing the uncertainty surrounding OIF? In light of looming challenges to the safety and efficacy of global-scale market based OIF, I present the case against commercialization in 5.2. In 5.3 I argue, however, that even if commercialization under compliance markets is deemed unacceptable by scientists and policymakers, continued research is vital to delegitimize future developments in voluntary carbon markets that, at present, may be incentivized to proceed contrary to the precautionary principle.

\subsection{The case against commercialization}

At a bare minimum, the commercialization of OIF can not be advisable unless it satisfies the standards established by existing international offset markets. The Clean Development Mechanism [Gillenwater and Seres 2011], developed under the Kyoto Protocol, has become the 136 
preeminent international offset program and has set basic guidelines for qualifying projects. Eligible projects must be, amongst other stipulations, permanent, additional, free of leakage, and amenable to monitoring [Gillenwater and Seres 2011]. In the context of OIF this amounts to two basic questions. First, does it work? That is, will fertilizing iron-limited swaths of the ocean stimulate new ("additional") production that will sequester carbon into the deep ocean for long periods ("permanent") without leading to downstream reductions in productivity ("leakage") triggered by upstream macro-nutrient utilization (5.2.1)? Second, can it be measured? That is, can the net additional carbon sequestration from an individual OIF project be quantified accurately enough to ensure fair and consistent compensation (5.2.2)? Finally, although not explicitly mentioned in current market frameworks, a critical third question is implicit in any geoengineering project of this scale. Is it safe from adverse, unpredictable side effects (5.2.3)? The litany of challenges, risks, and lingering uncertainty that beguile each of these questions is detailed respectively in 5.2.1, 5.2.2 and 5.2.3 (see Fig. 5-2). In Section 5.2.4 I conclude that safe, predictable, and effective management of OIF on emerging offset markets is simply untenable.

\subsubsection{Will it work?}

\section{Does iron fertilization stimulate new production?}

After Martin's Iron Hypothesis was proposed, early shipboard incubations [Martin et al. 1991] began to provide compelling evidence for iron limitation. Although these incubations were plagued by methodical biases, the results were enough to prompt an era of large scale, in-situ, iron enrichment experiments [Chisholm and Morel 1991]. Since 1990, 13 mesoscale artificial enrichment experiments have been performed, with 7 located in the Southern Ocean.

These experiments were all carried out in a similar manner. Iron, dissolved into acidified seawater, was dumped into HNLC surface waters, while the concurrent addition of $\mathrm{SH}_{6}$, a biologically inert chemical tracer, allowed the water parcel to be traced and observed in a Lagrangian framework for the following 10-40 days [Boyd et al. 2007]. The ensuing biogeochemical response consistently showed an increase in photosynthetic efficiency, chlorophyll concentration, primary production and a corresponding drawdown of $p \mathrm{CO}_{2}$ and macro-nutrients [Yoon et al. 2016]. Collectively, combined with observations of naturally enriched waters [de Baar et al. 2005], results have demonstrated fairly convincingly that iron fertilization does stimulate local productivity in certain HNLC regions [Boyd et al. 2007; Yoon et al. 2016].

Note, however, that the timing and location of these relatively small-scale experiments was carefully chosen. Blooms can still be limited, or co-limited, by light, silicate, macro-nutrients and grazing, leading to variability in the efficiency of iron fertilization. Discrepancies in local environmental, physical and seasonal conditions have been shown to have a pronounced effect 137 
on the relative success of any given particular patch fertilization [Boyd et al. 2007]. This means that to achieve even conservative estimates of stimulated growth, fertilization sites would need to be chosen with care and precision. In a dynamic ocean environment, this might not be practical, or possible, at the global scale.

\section{Is stimulated primary production routed into export production?}

Even if mass fertilization is scalable, it is critical to remember that iron limitation is only the first step of the Iron Hypothesis [Buesseler et al. 2008b]. Leveraging this insight into a sustainable global carbon sequestration program hinges largely on ambiguity over the long term fate of the new organic matter that is produced.

The stimulation of new production is only relevant to the medium-to-long term global carbon cycle if it is in turn sequestered for significant time scales. Most organic matter is however rapidly remineralized as it is respired in the surface ocean. This carbon is returned to the pCO2 pool and able to re-equilibrate with the atmosphere. For OIF to be a viable sequestration pathway a significant fraction of stimulated production must instead be exported out of the surface ocean to depth.

Some naturally fertilized systems have been observed to yield reasonably strong export fluxes relative to nearby iron depleted water[Blain et al. 2007; Pollard et al. 2009], however, it is problematic to extrapolate natural laboratories to large scale geoengineering efforts, or even patch fertilizations. Only EIFEX [Smetacek et al. 2012], one of 13 total mesoscale enrichment experiments, actually observed an increase in export [Yoon et al. 2016]. Localized naturally fertilized regions represent a highly specific response to a particular set of environmental conditions that may not be easy or practical to replicate across the Southern Ocean [Salter et al. 2012]. For instance, in natural systems iron is generally slowly and continuously supplied throughout the year, whereas in artificial fertilizations iron is deposited in pulsed rapid inputs. This results in a substantial fraction of the deposited iron being lost to abiotic processes such as particle scavenging prior to biological uptake [Bowie et al. 2001].

Even if the results from EIFEX can be replicated at scale, recent modeling work has predicted an underwhelming ceiling for integrated export in a continuously fertilized ocean compared to earlier studies [Aumont and Bopp 2006]. Current model estimates suggest that HNLCs are not capable of exporting any more than several hundred million tons of $\mathrm{CO}_{2}$ per year [Buesseler et al. 2008b], a stark contrast to the roughly 10 billion tons humanity releases annually [IPCC AR4 2007]. 


\section{Does export translate into long term storage?}

Regardless of export efficiency out of the surface ocean, only a very small fraction $(\sim<1 \%$ of total export [Prentice 2001]) of sinking organic matter will make it to the sediments where it can remain sequestered on geological time scales. The rest is remineralized at depth and eventually transported back to the surface ocean where it can be released into the atmosphere. The timescales over which this occurs vary with the remineralization depth [Gnanadesikan et al. 2003], but generally fall on the order of 10-100s of years. Geoengineering advocates contend that if a large enough export flux is achieved, then this is long enough to buy time until other longer term climate solutions are developed. Questions remain, however, if this relatively mild best case scenario is even attainable.

During EIFEX, the lone artificial OIF experiment that found evidence of increased export, Smetacek et al. [2012] concluded that over half of the stimulated bloom's biomass sank below 1000 meters. These results, however, must be considered in a broader context. The efficiency of the biological pump is highly variable on a regional and seasonal basis. On a seasonal basis, deep winter mixing which can penetrate hundreds of meters below the surface, could quickly return carbon remineralized relatively deep to the surface ocean. Regionally, targeting areas of deep water formation may increase the reminerialization depth as surface waters subduct, but may also introduce operational hazards from working in the coastal, heavily ice covered regions where deep water typically forms.

Further, some have pointed out that the export flux and variability in the remineralization depth can not alone describe oceanic carbon storage, pointing instead to preformed nutrient budgets which are additionally controlled by stoichiometry and circulation [Gnanadesikan and Marinov 2008]. In this context, much larger space and time scales must be considered to quantify carbon storage, dramatically complicating the scalability and extrapolation of localized enrichment experiments.

\section{Do secondary effects on ecosystem structure effect the net sequestration of carbon?}

Southern Ocean iron enrichment experiments tend to preferentially increase the growth rates of diatoms, shifting community composition from smaller phytoplankton functional types to larger, silicate shelled, chain forming, diatom assemblages [Hoffmann et al. 2006; Hutchins and Bruland 1998]. The stimulation of these rapidly sinking, heavy assemblages is key to increasing the efficiency of the biological pump [Hoffmann et al. 2006] but might additionally trigger less desirable secondary consequences.

Many of the heterotrophic zooplankton, such as copepods, that preferentially graze on diatoms form calcium carbonate $\left(\mathrm{CaCO}_{3}\right)$ shells [Tsuda et al. 2007]. The precipitation of $\mathrm{CaCO}_{3}$ triggers a change in the speciation of the equilibrated carbonate system which leads 139 
to an increase in $\mathrm{pCO}_{2}$ [Frankignoulle et al. 1994]. Although the dissolution of CaCO3 is in turn an effective sink for $p \mathrm{CO}_{2}$, if it is first transported to depth calcification can act as a $\mathrm{CO} 2$ source on time scales of 10-100s of years. This process is known as the carbonate pump and has been observed to reduce the carbon sequestration capacity of natural systems by as much as $30 \%$ [Salter et al. 2014]. If a stimulated diatom population preferentially favors calcifying grazers, indirect stimulation of the carbonate pump ( $\mathrm{CO}_{2}$ source $)$ must be weighed against stimulation the biological pump $\left(\mathrm{CO}_{2}\right.$ sink).

More generally, increased primary production may not dictate a proportionate response in export production. Population size is not only regulated by bottom-up controls on phytoplankton growth rates such as light and nutrient (e.g. iron) limitation, but also by top-down controls on phytoplankton loss rates imposed predominately by grazers [Behrenfeld et al. 2013]. If highly stimulated growth rates improve grazing efficiency, a negative feedback loop could emerge in which increasing grazing rates damp population gains [Rohr et al. 2017]. As carbon is transferred up the food chain, and partially respired along the way, increased grazing would in turn lead to less efficient carbon export [Boyd and Doney 2003].

\section{Does local macro-nutrient utilization compromise downstream productivity?}

Even if long term, local sequestration was successful, in a highly interconnected global ocean any local perturbation must be considered in the context of its ensuing downstream, non-local effects. By design, successful OIF would drawdown previously unused macro-nutrients and trap them at depth. While these unutilized macronutrients were previously of little use in iron limited surface waters, the depression of the nutrient profile leaves intermediate waters with reduced nutrient concentrations as well. These intermediate waters are eventually advected and upwelled at lower latitudes. To understand the net effect of Southern Ocean OIF we must also understand the price of reducing the supply of downstream macro-nutrients. That is, what is the cost of leakage?

Unfortunately, this question transcends the scope of modern observational capabilities. Several modelling studies, however, have suggested that leakage could be considerable, leading to a significant downstream reduction in primary production, atmospheric $\mathrm{CO}_{2}$ drawdown and export production in the tropics [Aumont and Bopp 2006; Gnanadesikan et al. 2003; Oschlies et al. 2010; Sarmiento and Orr 1991]. Specifically, Oschlies et al. [2010] found that when integrated over 100 years, increased non-local outgassing compromised the net $\mathrm{CO}_{2}$ drawdown by $20 \%$. Worse, Gnanadesikan et al. [2003] found that the integrated non-local reduction in export was 30 times greater than locally stimulated export. In turn, after 100 years only $2-44 \%$ of the initially stimulated local export remained removed from the atmosphere. While nonlocal productivity could be damped for hundreds of years, the majority of artificially added 
iron is likely to be rapidly removed from the water column and buried in the sediments via particle scavenging [Aumont and Bopp 2006], meaning the local stimulus could be short-lived relative to the non-local ramifications.

\subsubsection{Auditing: Can it be measured?}

A robust auditing framework is a prerequisite for any reputable OIF plan and hinges largely on the ability to establish accurate and reliable estimates of sequestration. This is challenging for any carbon sequestration scheme but is particularly problematic for OIF. Tremendous spatialtemporal variability in the stimulated efficiency of the biological pump prevents the simple extrapolation from iron input to carbon sequestration. At best we can attempt to directly measure the induced export flux and infer net sequestration from there. Unfortunately, the dynamic nature of the global ocean not only severely complicates measurements of local export but requires the complete consideration of non-local effects.

\section{Challenges measuring local export}

Export production is notoriously difficult to measure. Physical methods such as sediment traps which simply catch particulate "rain" are subject to, amongst other things, grazing by passing zooplankton and hydrodynamic biases over the mouth of the trap [Buesseler et al. 2007]. Chemical methods measuring the secular disequilibrium between particle reactive ${ }^{234} \mathrm{Th}$ and its conservative, long lived parent radioisotope ${ }^{238} \mathrm{U}$ provide a good proxy for export production [Buesseler 1998] but are subject to their own problematic biases and assumptions. By providing multiple lines of evidence EIFEX [Smetacek et al. 2012] was able to convincingly conclude that they induced an increase in local export production, but employing a similarly large suite of measurement tools would hardly be practical in a global scale OIF framework. Anything less, however, may not be reliable.

\section{Biogeochemical additionally and establishing a baseline}

Additionality, the notion that offset credits should not be granted for sequestration that would have happened irrespective of a proposed project, is often only considered in an operational context [Leinen 2008], but for OIF, a process designed to amplify a natural phenomena, it must also be considered in a biogeochemical context. In order to accurately audit it necessary is to establish a baseline by which to quantify how much additional export has been stimulated beyond what would have occurred naturally. Establishing such a baseline would require nearly continuous control measurements over the entire lifetime of the bloom at multiple locations throughout the surrounding unfertilized waters. Even then, separating the OIF induced signal 
from high frequency spatial-temporal variability, inter-annual variability and long term climate trends would prove nearly impossible [Cullen and Boyd 2008].

EIFEX [Smetacek et al. 2012] cleverly fertilized a water mass trapped in the interior of a large eddy to help control for mixing biases between the fertilized and control patch, but likely introduced new biases as well. Internal eddy dynamics are capable of modifying the insitu iron flux [McGillicuddy 2016] and accounting for a heightened export flux independent of the stimulus from artificial fertilization. Because the strength and direction of these internal dynamics vary between eddies [Gaube et al. 2014] adequately controlling for them would require the impossible task of measuring the same eddy, at the same time, with and without iron fertilization.

\section{Spatial/temporal dissonance}

Finally, overcoming the challenges hindering local export measurement may be irrelevant if the local signal does not dominate the net global signal. Ocean circulation and mixing increase spatial scales and distribute the effects of a local perturbation far from its point source, severely complicating long term verification and assessment [Buesseler et al. 2008b]. Non-local effects, largely triggered by the downstream depletion of macro-nutrients, are thought to be of a similar scale and often in an opposing direction to local effects [Aumont and Bopp 2006; Gnanadesikan et al. 2003; Oschlies et al. 2010; Sarmiento and Orr 1991]. Accurate auditing, then, would require estimates of both the locally induced export flux and consideration of all non-local effects [Yoon et al. 2016]. Unfortunately, large space and time scales prevent direct measurement of these effects11, while complex nonlinearities prevent reliable model-based estimates for individual deployments.

\subsubsection{Safety: Will it have adverse side effects?}

By design, OIF seeks to deliberately manipulate ocean biogeochemistry at the global scale. In a highly complex ocean system it is unreasonable to expect this will not lead to a bevy of broad ranging, unpredictable and unintended consequences. Given the breadth of the climate crisis, the prospect of marginal gains in carbon sequestration may reasonably outweigh the risk of collateral damage, however, there is first an obligation to understand the full scope of potentially harmful side effects and deem them acceptable [Buesseler et al. 2008b; Cullen and Boyd 2008].

\section{Anoxia and hypoxia}

If OIF is successful then increased export production will eventually fuel increased aerobic microbial decomposition at depth [Cullen and Boyd 2008]. Increased microbial decomposition 
will increasingly consume oxygen and could lead to the development of hypoxia or anoxia below the euphotic zone [Yoon et al. 2016]. These deoxygenated subsurface water can eventually be transported to the surface in coastal upwelling systems where they can trigger mass fish die offs [Cullen and Boyd 2008]. Similar events have been observed along the Pacific Eastern Boundary current [Chan et al. 2008; Grantham et al. 2004], and are thought to be triggered by non-local anthropogenic nutrient loading.

Simple early box-models predicted that large scale fertilization would create vast subsurface anoxic regions [Sarmiento and Orr 1991]. Later models countered that oxygen depletion may not be quite as severe, but only because the magnitude of the predicted sequestration flux also decreased [Denman 2008]. Similarly, compensating oxygenation has been predicted to occur at lower latitudes in some models, but only due to a reduction in productivity triggered by upstream nutrient utilization [Oschlies et al. 2010]. The net effect is difficult to constrain, but generally appears qualitatively opposed to the desired outcome of OIF; Net improvement in global export is tied to a net deterioration of subsurface oxygen.

\section{Broader ecosystem interactions - productivity, community composition, and fish- eries}

Despite strong evidence of an immediate, local increase in productivity following fertilization, some predict that on decadal timescales OIF will actually lead to a net reduction in global productivity triggered by a reduction in the downstream nutrient supply, particularly to the tropics [Aumont and Bopp 2006; Gnanadesikan et al. 2003; Zahariev et al. 2008]. Over long enough time scales a net reduction in primary productivity could ripple up the food web reducing the availability of harvestable fish stocks. Gnanadesikan et al. [2003] estimated that the cost to fisheries could be as high as $\$ 150$ per ton of carbon sequestered via OIF.

The ultimate effect on fisheries is further complicated by the potential for complex, unpredictable changes to ecosystem structure fueled by shifts in species composition at lower trophic levels [Chisholm and Morel 1991]. During sustained fertilization, blooms have been observed to shift to diatom dominance [Marchetti et al. 2006], and in turn favor larger species of zooplankton [Tsuda et al. 2006]. These changes in community composition have at times lead to an increase in the abundance of Pseudo-nitzschia, a diatom genus known to produce the harmful neuro-toxin domoic acid [Silver et al. 2010; Trick et al. 2010].

The precise community response, however, remains largely unpredictable. Even at smaller, experimental scales, ecosystems have been observed to respond differently to multiple fertilizations conducted at the same site [Boyd et al. 2007]. At a global scale these changes could lead to dramatic and unpredictable regime shifts in community composition and more generally regional biogeochemistry [Boyd and Doney 2003]. It is, at best, unclear how major changes 
in ecosystem structure will effect ocean resources and fisheries.

\section{Non-CO2 climate active gasses}

The net radiative effect of OIF may be significantly altered by modified contributions from non- $\mathrm{CO}_{2}$ climate active gasses, such as nitrous oxide $\left(\mathrm{N}_{2} \mathrm{O}\right)$, methane $\left(\mathrm{CH}_{4}\right)$, and dimethylsulfide $(D M S)$.

$\mathrm{N}_{2} \mathrm{O}$ is a greenhouse gas roughly 300 times more potent than $\mathrm{CO}_{2}$ on a per-molecule basis [Ramaswamy et al. 2001]. Oceanic $\mathrm{N}_{2} \mathrm{O}$ production is associated with both the bacterial oxidation of remineralized ammonium to nitrate, as well as the bacterial reminerialization of organic matter at low oxygen levels [Cohen and Gordon 1979]. The existence of multiple pathways complicates precise estimates of OIF induced $\mathrm{N}_{2} \mathrm{O}$ fluxes, but $\mathrm{N}_{2} \mathrm{O}$ production is generally thought to increase as increasing export is inevitably decomposed. Observations from the SOIREE iron enrichment experiment [Law 2008] in addition to modelling studies [Jin and Gruber 2003; Oschlies et al. 2010] have reported a net increase in $\mathrm{N}_{2} \mathrm{O}$ production estimated to compromise the net radiative effect of atmospheric $\mathrm{CO}_{2}$ removal by 5-10\%. EIFEX, one of the largest enrichment experiments to date, however, observed no detectable change in $\mathrm{N}_{2} \mathrm{O}$ production [Walter et al. 2005].

$\mathrm{CH}_{4}$, another greenhouse gas produced during microbial decomposition, is roughly 20 times more potent than $\mathrm{CO}_{2}$ [Ramaswamy et al. 2001]. Oceanic $\mathrm{CH}_{4}$ is produced predominately by bacteria in completely anoxic microhabitats associated with sinking particulate organic matter [Karl and Tilbrook 1994]. While increased export would increase the prevalence of these microhabitats, the net potential for OIF induced $\mathrm{CH}_{4}$ production to offset atmospheric $\mathrm{CO}_{2}$ reductions is not expected to exceed 1\% [Oschlies et al. 2010].

$D M S$, unlike $\mathrm{N}_{2} \mathrm{O}$ and $\mathrm{CH}_{4}$, is not a greenhouse gas. Instead, $D M S$ leads to the creation of sulfur aerosols which in turn help seed cloud formation, working to cool the atmosphere by increasing earth's albedo. Oceanic DMS is produced as byproduct by marine phytoplankton and has been proposed as a biological pathway for climate regulation [Charlson et al. 1987]. While the nature of this regulatory loop has been found substantially more complex than initially hypothesized [Ayres et al. 1997; Quinn and Bates 2011], it remains a significant link between marine biota and climate. The net effect of OIF on DMS production, however, is unclear. Some enrichment experiments have seen an increase in $D M S$ production immediately after fertilization [Liss et al. 2005; Turner et al. 2004, 1996], with hikes as high as 6.5fold [Turner et al. 2004]. Other experiments, however, have reported no change in DMS production [Nagao et al. 2009; Takeda and Tsuda 2005], or even observed a decrease following an initial spike [Levasseur et al. 2006].

Ultimately, without observations of patch-scale fertilizations longer than 1-2 months, no 
less for a continuous, basin wide fertilization program, the forcing on non- $\mathrm{CO}_{2}$ climate active gasses remains largely unknown [Law 2008]. Constraining these fluxes is critical to understanding the net radiative effect of OIF and will require a better understanding of changes to community composition, particulate export, and deep bacterial remineralization at time and space scales much larger than a single fertilization.

\section{Ocean acidification}

Finally, the desired uptake of $\mathrm{CO}_{2}$ into ocean will only exacerbate ocean acidification. Ocean acidification is caused as increasing $\mathrm{CO}_{2}$ shifts the equilibrium of the carbonate system in favor of an increasing concentration of $H+$ ions, thus reducing the $p H$. Ocean acidification has been widely shown to be detrimental to some marine biota, particularly calcifiers [Doney et al. 2009]. While the majority of anthropogenic $\mathrm{CO}_{2}$ may inevitably end up in the ocean regardless, if successful, OIF will undoubtedly increase the rate at which it is added, giving organisms less time to adapt [Denman 2008].

\subsubsection{Managing uncertainty and market failures}

Establishing any chance for a successful global scale OIF operation would call for careful consideration into when and where each individual fertilization is implemented [Buesseler et al. 2008b; Yoon et al. 2016]. Collectively optimizing a global portfolio of fertilization sites would require intensive monitoring coupled to a comprehensive adaptive management plan. Monitoring would need to account for local and non-local sequestration, a baseline by which to establish additionality, and a suite of complex side effects. Scientific decision making would need to be equipped to understand and react to unpredictable developments on time and space scales well beyond the scope of local fertilization [Gnanadesikan et al. 2003]. Implementation would need to be flexible enough to change course on the fly, but also incremental enough to safeguard against significantly time-lagged, down stream consequences.

Given the challenges associated with accurate measurement, developing such a demanding management program would be exceedingly difficult, even under unilateral authority. It might be impossible under market control. Institutional inefficiencies would dramatically hinder the ability of myriad independent private corporations to cohesively implement a complex and dynamic plan. At best, any practical approach to establishing a baseline and quantifying additionality would have to be done in a broad mean sense. This sort of generalization creates the exact problem that plagues other offset markets [Gillenwater 2012]. Corporations looking to maximize the differential between the baseline and outcome will be incentivized to seek underestimated baselines rather than improved outcomes. Even well intentioned incentives designed to ensure corporations fertilize the right places at the right times could be corrupted 
by challenges to enforcement and unreliable auditing. Finally, no project would be truly oceanographically independent, making it impossible to appropriately distribute the costs and benefits across operationally independent projects.

Ultimately, it is difficult to see how market forces could ensure OIF conforms to the best scientific judgment. Consequently, these market failures will compound the already substantial risk of adverse side effects and damp our ability to react to emerging threats, all while introducing problematic economic and legal ramifications. Economically, without a robust auditing framework to ensure fair and consistent compensation, corporations incentivized to game the system could undermine the entire global offset markets. Legally, if harmful consequences do arise, establishing liability will be nearly impossible across complex, multi-actor, non-local, time-lagged lines of etiology. Taken together, the inability of markets to properly manage the uncertainty and risk associated with OIF on a global scale or resolve the challenges imposed by tremendous spatial-temporal dissonance highlight the case against commercialization.

\subsection{The case for continued research}

While few scientists support commercialization, a much more controversial debate has ensued over the prudence of continued research into OIF. Given the tremendous concerns over commercialization many argue that further research would only misallocate finite scientific resources and bolster a moral hazard threatening to distract from more legitimate mitigation efforts.

On the other hand, the case for continued research is three-fold. First, from a basic research perspective, unraveling the role of iron in our oceans and its contribution to glacial-interglacial climate variability will help shape our understanding of ocean biogeochemistry and climate change. Second, it is not impossible that some realization of OIF could eventually be safely and thoughtfully implemented under unilateral governmental authority as one of many useful tools to address climate change, particularly if mitigation efforts continue to fail. Third, if there is reason to believe that commercialization could proceed contrary the scientific consensus, then continued research could help deter reckless behavior by delegitimizing unfounded commercial deployments. Argument three warrants further consideration, and in 5.3.1 I outline the incentives that could drive development on voluntary markets and the role that continued research can play moving forward.

\subsubsection{Prospects for commercialization on voluntary offset markets}

It is unlikely that any global governance framework would blatantly disregard scientific wisdom and begin granting offset credits for OIF under heavily regulated compliance offset markets 
(COMs), however, there are no such barriers to entry on voluntary offset markets (VOMs). VOMs differ from COMs, such as those implemented by the Kyoto Protocol, in that offsets are bought and sold without any federally mandated obligation. Trading on VOMs is instead motivated by a sense a of personal responsibly, corporate branding or an expectation of impending regulations. Lacking significant oversight, the VOMs could serve as a vital seed ground for the development of private OIF ventures.

\section{Size of the voluntary market}

Compared to the \$50-100 billion total value of COMs [Carbon Market Monitor 2016], VOMs pale in size. Still, with $63.4 \mathrm{MtCo}_{2}$ e traded in 2016 for a total of $\$ 191.3$ million [Hamrick and Gallant 2017], there is considerable room for a small company to secure lucrative profits. In 2016 , the average price for all transactions was $\$ 3.0 / t \mathrm{CO}_{2} \mathrm{e}$ but ranged wildly from $\$ 0.5 / t C \mathrm{O}_{2} \mathrm{e}$ to as high as $\$ 50.0 / \mathrm{tCO}_{2} e$. Despite some volatility in market size (demand rose by $10 \%$ in 2015 [Hamrick and Goldstein 2016] but dropped off by 24\% in 2016 [Hamrick and Gallant 2017]) and the growth of COMs in the wake of COP 21, there is an expectation that VOMs will remain viable with the overall demand for sustainable development and industry interest in carbon neutrality is on the rise [Hamrick and Gallant 2017].

\section{Perception of low cost alternative}

Relative to other carbon offset projects, there is a perception that OIF is a substantially cheaper, economically viable solution [Keith et al. 2006; Worstall 2012]. Given the low cost of iron and the very high stoichiometric ratio with which $\mathrm{CO}_{2}$ is fixed and iron utilized, it is easy to understand how OIF can appear, at first glance, seductively affordable. Early projections estimated the cost of OIF as low as $\$ 1-2 / t C \mathrm{O}_{2}$ e [Markels and Barber 2002], leaving substantial room to profit over the average price currently being traded on VOMs.

Of course, the reality may be much less enticing. Early estimates were biased by problematic assumptions regarding export efficiency, downstream macro-nutrient depletion and $\mathrm{N}_{2} \mathrm{O} / \mathrm{CH}_{4}$ compensatory fluxes, and did not internalize the true cost of research and development, monitoring, or delivery systems at the global scale [Watson et al. 2008]. Revised projections generally range from $\$ 8-80 / \mathrm{tCO}_{2} e$ [Boyd 2008], but still typically exclude the price of potentially harmful externalities which have been estimated as high as $\$ 150 / t C \mathrm{O}_{2} \mathrm{e}$ to fisheries alone [Gnanadesikan et al. 2003]. One, more extreme, estimate argues for an almost certainly prohibitory price of $\$ 457 / t \mathrm{CO}_{2}$ e [Harrison 2013]. Perhaps most accurately though, in light of the vast uncertainty in what we can infer from patch scale fertilizations and model integrations, the truth is that we just do not know how expensive long term iron induced sequestration might be at a global scale [Barker and Bashmakov 2007]. 
What we do know, however, is that it is not very expensive to dump iron into the ocean. Even if the cost of sequestration in a comprehensive, credible OIF scheme is prohibitory, the operational start up cost for small-scale, speculative operators is not. Whether these deployments could ever actually sequester what they claim remains uncertain, but it is precisely this uncertainty that creates the opportunity to discount the risk portfolio and overvalue the chance to win big. More research is needed to constrain the true cost of sequestration and delegitimize the idea that it can be easily extrapolated from one-off, patch-scale, fertilization experiments.

\section{Investing in the future and establishing intellectual property}

Without a firm grasp of the true cost of sequestration and buttressed by the low price of iron and overhead, there is a reasonable economic argument for small-scale operators to invest in the development of OIF. Even if the odds for success are slim, the stakes are low (economically if not environmentally) and the jackpot is huge. In the most optimistic, albeit unlikely, scenarios, OIF could generate billions of dollars worth of offsets [Cullen and Boyd 2008], fundamentally disrupting carbon markets [Neeff 2007]. Relative to the low cost of investment, the upside is high enough that even if OIF is not immediately profitable it could be justified as a shrewd investment.

It is exactly these sort of financially low risk, high reward ventures that appear poised for success on VOMs, which are seen as promisingly fertile soil to test emerging carbon sequestration technologies [Hamrick and Gallant 2017; Hamrick and Goldstein 2016]. In the hope that one day OIF will be adopted into compliance markets, it is relatively affordable for entrepreneurs to stage preliminary development on VOMs with an eye to test methodologies and preemptively establish intellectual property. If further research can clarify the biogeochemical impediments to a market-based OIF approach and diminish the perception that it will ever be viable on COMs, it will deter speculative investment on VOMs.

\section{Regulatory and legal framework}

Exploratory development on VOMs benefits from a loose regulatory environment with no federally mandated oversight. While a suite of standardization bodies have emerged to administer credibility by verifying that projects are, in fact, permanent, additional, and free of leakage [Hamrick and Gallant 2017], it is unclear how a collection of independent auditors would cohesively overcome immense monitoring challenges to develop a comprehensive OIF validation scheme. It is more likely that commercial operators would shop between diverse auditing options before settling on the most economically favorable framework. Even if more reputable standards bodies refuse to accredit OIF, there is nothing blocking the emergence of 
new organizations willing to do so, and only the scientific community would be equipped to discredit them. This regulatory flexibility relaxes the burden of proof that should be expected from commercial operators and could encourage reckless development.

In theory, the United Nations Convention on the Law of the Seas and the London Convention, which generally ban dumping on the high seas, should prevent overtly harmful operations. Unfortunately when if comes to OIF, international jurisdiction is often vague and difficult to enforce [Bertram 2011]. In 2008, the London Protocol, with support from the International Maritime Organization [IMO 2008], banned commercial fertilization [LC-LP.1 2008], but ambiguity arose over what constituted a permissible, legitimate scientific activity[Bertram 2011; Goodell 2011a]. In 2013, the UN formally recognized OIF as geoengineering and mandated stricter environmental assessments and approval prior to permitting [LC-LP.4 2013], but questions remain over the capacity to enforce international law in the remote Southern Ocean without any centralized legal authority. Poor enforcement and protections under the guise of science have long sheltered the whaling industry [Mangel 2016] and could potentially do the same for OIF. Without legitimate research programs for reference, it could be even easier for commercial operations to feign scientific legitimacy.

\section{Ethical arguments and public perception}

OIF can only leverage a loose regulatory and legal framework if there is a market to support it. The question of whether there is demand for legally and scientifically suspect offset credits, however, might not be a strictly economic one. Absent any formal obligations, buyers on VOMs are often driven by an earnest desire to do what is right. Given the increasing threat of climate change and the deteriorating state of ocean health, it is not difficult to see how OIF could be sold to a less informed public as an ethically viable gamble.

By fixating only on favorable outcomes, OIF can and has been spun as marine forestation [Goodell 2011b]; A global gardening project to support ecosystem health, boost global fisheries, and help feed the world, all while sequestering billions of tons of carbon. Buyers are increasingly interested in these sort of social and environmental co-benefits and are willing to pay for them [Hamrick and Goldstein 2016]. Terrestrial analogs like forestry and land use projects promise similar protection to ecosystem services and successfully captured the second largest market share at the highest average trading price on VOMs in 2016. Together, they were three times more valuable than renewables [Hamrick and Goldstein 2016]. Current scientific wisdom suggests this comparison is not justified, but without continued research, commercial operators may be able to leverage lingering uncertainty to cast OIF in a positive light and shape public opinion, and in a buyers market, where there is no obligation to participate, public perception is tantamount to value. 


\section{Early Case Studies}

By the mid-late 2000s start ups championing OIF had gained considerable momentum. Planktos, founded at the turn of the century by Russ George with an expressed intent to "save the world and make a little cash on the side", had acquired an oceanographic vessel [Goodell 2011c]. GreenSea Ventures, a Virginia based outfit, had begun to establish intellectual property by securing patents for several iron delivery strategies [Bowie et al. 2016]. Most notably, Climos, had acquired 3.5 million dollars in series A venture capital funding and the support of Elon Musk ["New Plankton-Seeding Venture Reaps \$3.5 Million" 2008]. The reputability of these organizations ranged from a pirate-like disregard for scientific nuance at Planktos [Goodell 2011c], to an active engagement with the scientific community at Climos [Leinen 2008].

By the end of the decade, however, the scientific consensus had begun to crystallize; It was too early to support the commercialization of OIF [Buesseler et al. 2008b]. With the increasingly negative perception coupled to legal challenges introduced by the London Protocol [LC-LP.1 2008], the tide began turn for the first wave of OIF start ups. In 2008 Planktos was forced to halt operations mid deployment after investors jumped ship, and shutdown all together shortly there after. Climos, lasted longer but ultimately could not withstand the legal and scientific scrutiny, eventually shifting their focus towards broader geoengineering technologies [Goodell 2011d] before seeming to disappear completely.

Nevertheless, the threat of commercial development has not receded entirely. In 2012 Russ George reemerged to dump some 100 tons of iron sulphate into the Pacific Ocean [Lukacs 2012]. More recently, in 2017, the Oceaneos Marine Research Foundation sparked controversy when it began to seek permits to dump 10 tons of iron off the Chilean coast [Tollefson 2017]. If long term mitigation efforts continue to fail, it is unlikely that commercial interest in OIF will disappear on its own. Further research, is needed to continue to unravel the complexity, uncertainty and risk inherent in a global scale OIF platform and highlight the challenges to market based management the should preclude commercialization from consideration.

\subsection{Conclusions}

The prospects for a successful, basin wide, OIF campaign are shrouded in uncertainty and appear dubious at best. Even if iron is able to stimulate productivity at scale in HNLCs it is unclear how much will be exported to depth to be sequestered for adequate time scales and to what degree complex, non-linear, feedbacks into ecosystem structure and downstream macronutrient utilization might compromise net atmospheric $\mathrm{CO}_{2}$ drawdown. Worse, we don't fully understand how deliberately manipulating ocean biogeochemistry at this scale could threaten intricate marine ecosystems, global fisheries, or the flux of non- $\mathrm{CO}_{2}$ climate active gases. 
Nevertheless, the climate crisis is not going to disappear and all potential contributions to a solution warrant thorough consideration. Risk and uncertainty will weigh heavily in all options and must not alone preclude any from consideration. Success, then, will be largely predicated on selecting strategies that can optimize our ability to manage uncertainty and improve our preparation to adapt to unpredictable developments along the way. It is resoundingly clear that a market-based approach to OIF will do neither.

As continual fertilization induces an increasingly non-local and time-lagged response across the ocean it will become impossible to accurately distribute responsibility across many independent operations. The inability to attribute the true and total consequences of any individual fertilization, compounded by the uncertainty associated with measuring even the net effect of all integrated deployments, derails any hope for reliable auditing. Without a robust auditing framework or serviceable enforcement, misaligned incentives will prevent market forces from aligning with a rapidly evolving fertilization scheme and compromise our ability to react and adapt to unpredictable developments beneath a cloud of uncertainty.

If OIF was ever to be implemented, likely as a last ditch effort, it should be under unilateral authority and with dynamic management. Continued, incremental, research will not only clarify the viability of a potential emergency deployment, but is, perhaps more importantly, critical in deterring the reckless development of OIF on VOMs. Without consumer confidence that offsets are meaningful, there is no financial or ethical imperative to drive demand on VOMs. As it stands, the uncertainty surrounding OIF is large enough to justify a diversity of public opinion, particularly if developers highlight only positive outcomes. Further research is needed to reduce uncertainty and constrain public perception by continuing to clarify the risks, elaborate the challenges, and delegitimize the promise of an iron bullet. 


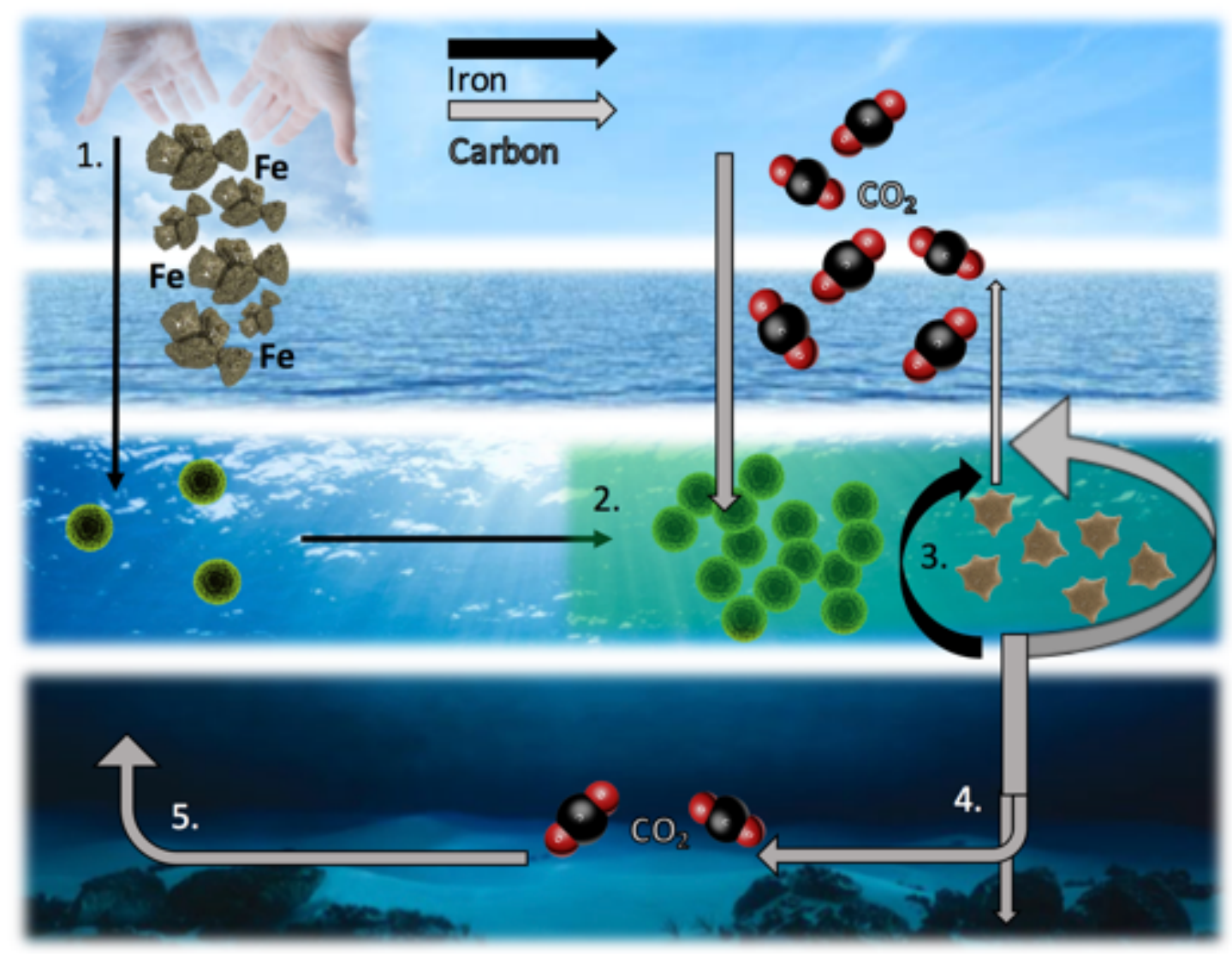

Figure 5-1: Ocean iron fertilization schematic. 1. Iron is added to High Nutrient Low Chlorophyll Regions (HNLCs, i.e. Southern Ocean) where phytoplankton growth is limited by iron availability. 2. Iron stress is relieved, division rates boom, phytoplankton bloom is triggered, drawing down increased $\mathrm{CO} 2$ through photosynthesis. 3. After Phytoplankton die, or are consumed, most are remineralized at the surface, recycling nutrients and Carbon. 4. Others sink to depth before being remineralized in the deep ocean on in marine sediments. 5. $\mathrm{CO}_{2}$ is sequestered at depth for 100s of year before being upwelled to surface (or much longer in sediments) 


\section{The Case Against Commercialization}

\section{CDM Protocol}

The Clean Development Mechanism, the preeminent international offset program, requires that all eligible projects MUST BE

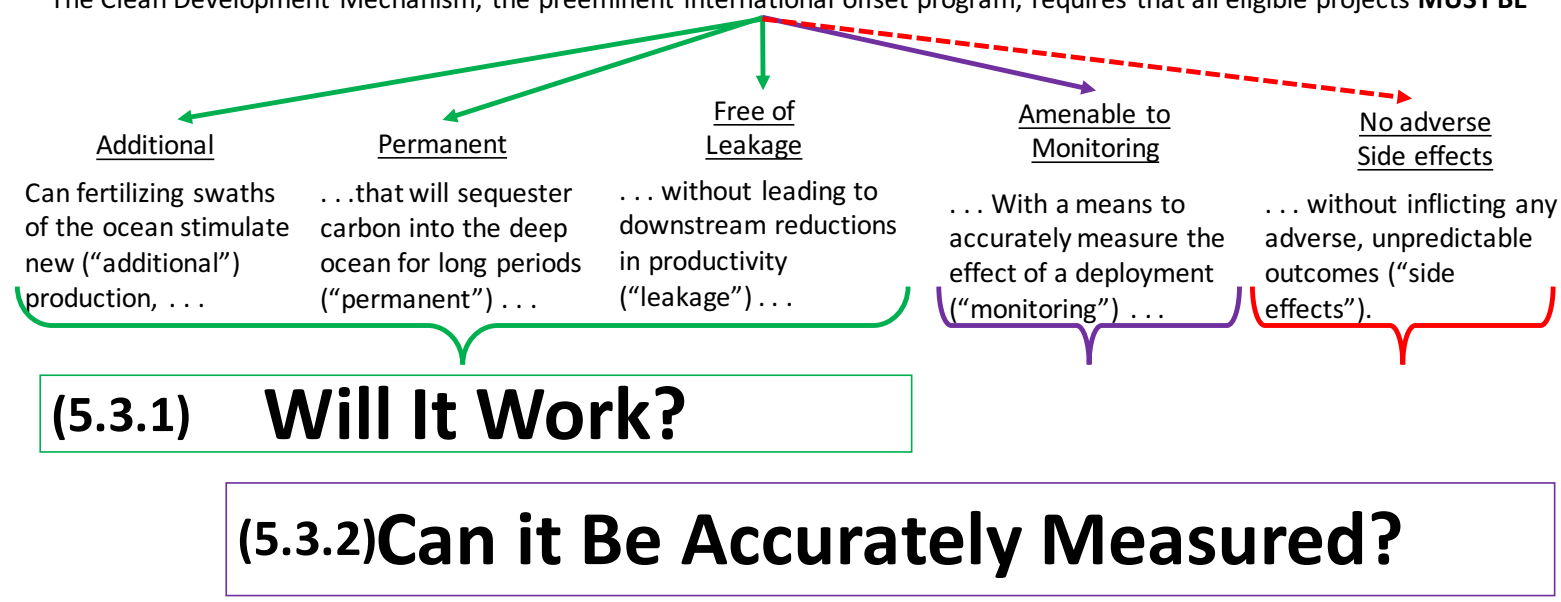

\section{(5.3.3) Is it Safe?}

Figure 5-2: Flow Chart: The case against commercialization. In the context of the Clean Development Mechanism, a viable commercialization strategy must be, additional, permanent, free of leakage, and amenable to monitoring. Logically, any project must also be free of adverse side effects. In the context of SO OIF this amounts to three questions: Will it work (5.2.1), can it be measured (5.2.2) and is it safe (5.3.3)? 
Chapter 6

\section{Conclusion}




\subsection{General results}

Southern Ocean phytoplankton distributions are characterized by short seasonal blooms and pronounced spatio-temporal variability. Harsh light limitation, nutrient stress and grazing pressure severely suppress net population growth for most of the year until one, or more, of these constraints is sufficiently relieved by physical processes for long enough to sustain growth. The canonical wisdom has held that phytoplankton blooms occur when mixed layer shoaling sufficiently relieves population-averaged light stress, but we find ample evidence of net population growth fueled by improved iron availability and/or reduced grazing pressure as well. Seasonal light limitation still plays a critical role, but it is clear that it can be complimented, and sometimes dominated, by other physical processes that drive net population in different ways, sometimes from the bottom-up, via processes that modify division rates, and sometimes from the top-down, via processes that modify grazing rates.

In the simulated Southern Ocean this is evident in the variability of the relative timing between peak annual division rates and net population growth rates. In many cases, peak net population growth is, in fact, concurrent with shallow well-lit mixed layers and annually optimized division rates, indicating a conventional bottom-up, light limited, framework. Elsewhere, however, peak net population growth occurs while the mixed layer is still deep and division rates are weak, but the population is diluted enough that loss rates, fueled by inefficient grazing, are even weaker. This is consistent with observations of bloom initiation during deep mixing in the North Atlantic and the recently developed 'disturbance-recovery' hypothesis. As in the North Atlantic, results presented here from the Southern Ocean are also supported by remote sensing observations. Further, deep winter mixing and subsequent shoaling are not the only ways to trigger net population growth. In the marginal ice zone, for example, seasonal sea-ice can decouple division rates from loss rates by starving zooplankton in the winter before improving light and iron availability once it retreats. These results should not diminish the importance of bottom-up controls, as even in the dilution scenario described above low division rates must be sustained above grazing rates through a surplus of iron from depth, but rather are intended to stress that net population growth represents a subtle disequilibria between division rates and loss rates which can become decoupled in a variety of ways.

At the mesoscale, eddies are ubiquitous across the Southern Ocean and also capable of modifying biomass. Mesoscale variability is well associated with variability in the chlorophyll field, but our understanding of how eddies affect the biology inside them is complicated by a host of competing processes that act simultaneously, often in competing direction. It is important to distinguish between these underlying mechanisms because the source of anomalous eddy-induced biomass could have profound implications on larger-scale biogeochemical 156 
cycling, depending on whether eddies are inducing/suppressing new production or simply moving biomass around. Results from the analysis of over 10,000 eddies tracked in a high resolution, eddy resolving run of the Community Earth System Model show that eddies do in fact modify division rates, but also drive biomass in other ways. On average, simulated anticyclones stimulate division rates by upwelling iron through eddy-induced Ekman pumping. Typically, elevated division rates lead to elevated net population growth, indicating a strong bottom-up control, and eventually a biomass anomaly which can exceed coincident climatologic values by $10-20 \%$ in the spring ACC. Other processes, such as exacerbated light stress via mixing, relieved grazing pressure via dilution, and various modes of physical transport such as rotational stirring, trapping, and the vertical advection of biomass, however, all appear to play a regionally and seasonally variable role as well.

All together, it is clear that a confluence of competing physical processes complicates our understanding of what drives variability in Southern Ocean net community productivity. Lingering uncertainty in the relative dominance of different mechanistic drivers stresses the need for continued research and underscores the notion that trying to artificially manipulate them is at best ill-advised. Although seductive calls to curb atmospheric $\mathrm{CO}_{2}$ concentrations by fertilizing the Southern Ocean with iron have garnered considerable attention, a careful look at the science reiterates that concerns over efficacy, safety, and measurement prohibit a viable commercialization strategy. Continued research into the matter, however, is warranted to both improve our basic understanding of the system and delegitimize efforts to undermine the precautionary principle and commercialize iron fertilization on loosely regulated voluntary markets.

\subsection{Comments on modelling limitations}

As with an modelling study, it is important to consider these results in the context of the specific numerical framework employed and the limitations of how biogeochemistry is represented. Specifically, the implications of the phytoplankton scheme, the zooplankton scheme, and the lack of biogeochemistry in the sea-ice component warrant particular consideration.

\subsubsection{Phytoplankton scheme}

The phytoplankton scheme is notably limited by community composition, explicitly resolving only three classes of phytoplankton. Observationally, diatoms are thought to dominate most large blooms in the Southern Ocean [Brzezinski et al. 2001] as well as in Antarctic waters, while smaller phytoplankton become more prevalent further north [Iida and Odate 2014] and non-diatom blooms can emerge in coastal waters [KavanaughEffectcontinentalshelf2015; 
Schoemann et al. 2005]. Both diatoms and a small phytoplankton classification are resolved in CESM; however, Phaeocystis, a non-calcifying haptophyte important in the marginal ice zone [Arrigo et al. 1998], is not. Phaeocystis have been shown in the field [Tang et al. 2009] and incubations [Moore et al. 2007] to do well under low light conditions and proven capable of dominating blooms typically thought to be dominated by diatoms [DiTullio et al. 2000], particularly below ice or during early spring when the mixed layer is deep and community light availability is low [Arrigo et al. 1999]. However, despite faster growth at lower irradiance than diatoms, Phaeocystis have a relatively low iron uptake efficiency and tend to bloom only when iron concentrations are relatively high, typically limiting large blooms to nutrient replete coastal waters [Schoemann et al. 2005].

To better understand the implication of resolving Phaeocystis, Wang and Moore [2011] integrated CESM with Phaeocystis explicitly included using a steeper initial P-I slope but larger Fe half saturation constant than diatoms. Adding Phaeocystis did not dramatically effect bulk phytoplankton productivity in the simulation but did alter community composition, with Phaeocystis accounting for $13 \%$ of total primary productivity and $40 \%$ of primary productivity in coastal waters [BuitenhuisMAREDATworldatlas2013; Wang and Moore 2011]. It is unlikely that Phaeocystis would out compete diatoms in most iron deplete off shore regions of the Southern Ocean (i.e Bins P1 and P2 in Chapter 2 and cyclones in Chapter 3 and 4); however, if iron is sufficiently elevated then Phaeocystis may be favored, especially if light is low. Thus if Phaeocystis were included in this simulation it is possible they could dominate the early spring bloom seen in Bin A1 in Chapter 2 or in ACC anticyclones in Chapters 3 and 4, where mixing is deep, light is low, and iron is elevated.

While resolving Phaeocystis could alter community composition it is unlikely to effect the qualitative nature of the mechanisms described at the core of this analysis. If anything, Phaeocystis would provide an avenue for increasing population averaged division rates in deep, poorly lit mixed layers, which would actually increase the strength of the dilution-decoupling mechanisms described in Chapter 2 as well as the ability for anticyclones to inflate divisions rates described in Chapter 3. In the context of export, however, differences in size, stoichiometry, and export efficiency [Arrigo et al. 1999; Asper and Smith 1999] associated with changes in community composition are doubtlessly relevant to the efficiency of the local biological pump and microbial loop. Historically, export has been thought to be dominated by large, rapidly sinking, diatom assemblages [Armstrong et al. 2001; Michaels and Silver 1988], suggesting that a shift away from diatoms, as might be expected in anticyclones or iron rich coastal regions, would reduce export efficiency [Laws et al. 2000], however, the ubiquitous dominance of diatoms in driving export has been recently challenged [Cassar et al. 2015] and reduced efficiency could be compensated for by increased net primary production (NPP). In turn, the specific effects of community composition on bulk export warrants further investiga158 
tion in the context of both what phytoplankton groups are resolved and how they are modified by the mechanisms studied here.

\subsubsection{Zooplankton scheme}

The zooplankton scheme features a single class of zooplankton that differentially grazes on the three phytoplankton pools with a non-linear Hollings III functional form [Holling 1959]. The Hollings III functional relationship ensures that predation is depressed at low prey abundance and saturates at to a maximum at high prey abundance. This framework is focused on representing a realistic trophic control on phytoplankton and adequately resolving global nutrient and chlorophyll fields, but not necessarily representing a realistic and diverse zooplankton population. Never the less, zooplankton population dynamics feedback to control bloom size, phytoplankton community composition and the biological pump from the top down. In turn, to understand the implications of the zooplankton scheme on the strength of top-down controls it is important to consider the limitations of both the simulated grazing framework and community composition.

With regard to the grazing framework, although most earth systems models employ a Holling II or Holling III formulation, the specific parameterization of grazing in global climate models is still a topic of ongoing debate [Laufkötter et al. 2015], particularly in the Southern Ocean where sparse in-situ observations of grazing rates exists. However, because both simulated population specific net growth rates [Boyd et al. 2001] and population specific division rates [Sakshaug and Holm-Hansen 1986; Smith et al. 1999; Spies 1987] are within the observed range, loss rates must implicitly be the right order of magnitude as well. Moreover, the nature of the mechanistic pathways described here hinges more on the grazing functional form than the precise quantitative parameterization. Non-linearities in the predator-prey relationship ensure that zooplankton become more efficient grazers as the concentration of phytoplankton increases. This is the key to seasonal variability in population specific grazing rates and the theoretical underpinning of dilution driven decoupling mechanisms. Employing a Hollings I or II functional form without non-linearities at low prey abundance would qualitatively undermine the possibility for dilution driven bloom initiation; however, there is little basis to do so. The ability for zooplankton to become more efficient grazers as prey abundance increases at low concentrations is thought to be key in maintaining realistic ecology [Adjou et al. 2012] and has long been a mainstay of biogeochemical modeling [Doney et al. 1996; Fasham et al. 1990]. Note, that while this grazing framework does not include discrete aspects of zooplankton life history like diapause [Fiksen 2000; Varpe 2012], it does ensure that grazing efficiency is dramatically reduced during the winter when zooplankton might be expected to be dormant. A more explicit inclusion of diapause, where grazing completely shuts down, would still induce 
a trohpic decoupling in the winter; however, the strength of this decoupling would no longer vary with the depth of the mixed layer or the degree of dilution.

With regard to community composition, or lack there of, it is important to note that all micro- and macrozooplankton are simulated as a single zooplankton pool. This could be problematic because each phytoplankton pool is assumed to have distinct size and physiology, thus it is reasonable that they should be grazed on by different species of zooplankton at different rates. To address this without explicitly adding additional zooplakton pools, the bulk zooplankton component grazes differentially on small phytoplankton, diatoms, and diazatrophs. Further, grazed biomass is routed differentially into DIC, DOC, and POC and eventually into the ballasting model which controls the vertical flux of particulate matter. For instance, diatoms are grazed at lower rates and more biomass is ensuingly routed to the sinking detritus flux after they are grazed, representing the role of larger predators such as copepods [Moore et al. 2004] and their ability to increase export production. This differential grazing scheme affords a relatively complex trophic control on the phytoplankton population at an affordable computational cost, allowing for higher spatial and temporal resolution.

The lack of zooplankton community resolution does, however, limit specific quantitative inferences that can be made from the simulation. Instead, this work focuses on the qualitative insights that remain critical to eventually understanding how blooms form and how NPP will respond to a changing climate. For instance, recent work by [Park et al. 2017] provides robust in-situ physiological evidence to argue that light, rather than iron, is the limiting factor for growth between two Amundsen Sea polynas, but dismiss grazing as a relevant control. This conclusion is based on similar grazing rates experimentally estimated [LandryEstimatinggrazingimpact1982] during the bloom maxima in each polyna, suggesting that grazing cannot account for the difference in bloom size. Results here, however, stress that trophic decoupling may have still occurred in the larger bloom, just much earlier during bloom inception. Further, in an inter-model comparison study Laufkötter et al. [2015] found that 4 out of 9 models predicted decreasing NPP under RCP 8.5 despite improving division rates resulting from declining biomass driven by stronger grazing forced by changing temperatures. The potential for temperature to vary the strength of trophic coupling is not addressed here, but the more general point that phenological and trophic coupling mismatches can regulate phytoplankton biomass (and thus NPP) from the top down [Laufkötter et al. 2015] is consistent with our results. Here, it is clear how dilution driven mechanisms in deep mixing events and eddies can help drive phytoplankton ecosystem dynamics from the top-down. Increasing trophic diversity would likely only strengthen this conclusion as increased variability in physiology and community interaction would likely introduce more non-linearities that could lead to physically induced decoupling between predator and prey. 


\subsubsection{Sea-ice biogeochemistry}

In the marginal ice zone, simulated sea-ice does not include biogeochemistry, thus cannot sequester iron or resolve in-ice algae. Iron that is atmospherically deposited above sea ice is input directly into the surface ocean. This has important implications on bloom phenology in seasonally ice covered waters. Wang et al. [2014] found that by including the explicit sequestration and subsequent release of iron in sea-ice that iron input from melt water served to increase phytoplankton productivity. This result is consistent with observations of ice-edge blooms triggered by an impulse of iron from melt water [Fennel et al. 2003; Lancelot et al. 2009; Sedwick and DiTullio 1997]. A similar inclusion in this simulation would shift and consolidate iron input, likely leading to an increase in the correlation between bloom size and ice severity seen in Chapter 2. Additionally including more complex sea-ice biogeochemistry in general circulation models is a newer and evolving field [Duarte et al. 2017]. Representing algae, for instance, in sea-ice would likely modify trophic coupling by providing a seed population for phytoplankton [Duarte et al. 2017] and a food source for overwintering zooplankton. This effect of trophic seeding is thought to be important in the Arctic [Duarte et al. 2017] while iron seeding may be more important in the Antarctic [Wang et al. 2014]. Including seaice biogeochemistry would likely only strengthen the conclusions that ecosystem dynamics in the modelled marginal ice zone are largely controlled by the seasonal ice cycle and that this contributes to Southern Ocean wide variability in the fundamental processes that control bloom phenology (see Chapter 2). Eddies in the marginal ice zone were not the focus of Chapters 3 and 4, due to a weaker agreement with theory and observations relative to open ocean eddies. A cursory analysis, however, reveals they exhibit a unique set of dynamics in the simulation, distinct from open ocean eddies, and warrant more focused observational and numerical investigation.

\subsection{Moving forward}

Moving forward, the 'disturbance-recovery' hypothesis warrants continued consideration with more focused consideration into the biases and assumptions associated with estimating depthaveraged population dynamics and prescribing simulated grazing. An accurate representation of depth-averaged population dynamics is essential to testing any dilution-driven mechanism as they are predicated on a decoupling between local concentrations and the depth-integrated inventory. Getting depth-integrated inventories right from remote sensing data is challenging; satellites can only see the surface ocean and the mixed layer depth estimates required to extrapolate surface concentrations are based on climatologies and re-analysis products. Getting at depth-integrated, population-averaged, biological rates is even more difficult, but critical 
as light and iron vary across the water column. Fortunately, in-situ alternatives, such as biooptically equipped ARGO floats, are becoming more prevalent and will need to be leveraged to ground truth the assumptions we use to extrapolate information about depth-averaged populations. Even in simulations, biogeochemical tracers are often not saved below $150 \mathrm{~m}$, as they are typically negligible below the euphotic zone. This is not true, however, during deep mixing events, which have been shown capable of initiating blooms by entraining nutrients and decoupling predator from prey. Simply extending vertical profiles of saved biogeochemical output will improve analysis. Finally, The degree to which a simulation can modify grazing efficiency is predicated on the prescribed grazing formulation. It will be important to test the sensitivity dilution-driven blooms to different grazing parameterizations and functionalities. This is particularly relevant in the Southern Ocean where in-situ observations of grazing rates are sparse.

At the mesoscale, it will be important to improve our ability to distinguish between anomalous biomass that was created by biological rate-based mechanisms and that which was transport across a gradient but did not modify net community production. Here, it was possible to explicitly examine anomalous biological rate terms in the simulation, but this is not as straight forward from space. Less direct methods must be used to infer the source of anomalous biomass in eddies from remote sensing data. One option that has been employed is to analyze the shape of the anomaly field across the horizontal breadth of an eddy. The idea is that lateral stirring should induce a strong dipole, whereas lateral trapping and/or ratebased processes should induce a strong monopole. The dominance of rate-based mechanisms can, therefore, be inferred from a monopole if the direction of the background gradient and eddy propagation is not such that trapping could induce an anomaly in the correct direction. This methodology, however, leaves many eddies under-constrained and can be complicated by different mechanisms perturbing the anomaly field simultaneously. In the future it would be useful to compare the surface profile of simulated eddies to the explicitly resolved rate terms within them to test how reliable these inferences are. Observationally, remote sensing data could also be compared to division rates estimated from the recently developed Carbon-based Productivity Model so long as care is taken to consider its associated assumptions as well.

Further, a better understanding of the mechanistic pathways that drive net population growth rate anomalies will help constrain where and when rate-based anomalies should be expected. The source of anomalous iron transport, for instance, is subject to a good deal of debate. New iron can be vertically supplied to phytoplankton in eddies by via mixing, eddy pumping, linear eddy-induced Ekman pumping, and nonlinear sub-mesoscale processes that act around an eddies periphery. Judgments about which processes dominate are often inferred from remote sensing data or informed by models that can not adequately resolve important sub-mesocale processes. Continued shipboard observations of the mixed layer depth 162 
and the vertical velocity field inside of eddies, combined with in-situ measurements of the iron profile and productivity, will go a along way towards constraining how iron is transported and consumed, and thus where and when anomalous iron and division rates should be expected.

More generally, over the coming decades, as oceans warm and ice melts, the physical and biogeochemical landscape of the Southern Ocean is certain to change. Continued research into the mechanisms that drive phytoplankton growth will help us understand how a shifting environment will alter net primary productivity, carbon export, and ocean biogeochemistry writ large. Understanding these changes could ultimately help us understand if the oceans will buffer, or amplify, a changing climate. As models begin to resolve more and more processes at finer resolution and in-situ observations are made easier and easier with the aid of autonomous instruments, modellers and observationalists will need to increasingly work together to understand these changes as they unfold in real time. 
APPENDIX A

\section{CESM SURFACE CONCENTRATION CORRECTION MODEL}




\section{A.1 Introduction}

Due to computational storage capacity limitations certain variables were only saved as monthly averages. Supplementary Material S1 details the diagnostic model used to approximate surface phytoplankton carbon concentrations for calculations at daily resolution

\section{A.2 CESM surface concentration correction factor}

Due to computational storage capacity limitations surface phytoplankton carbon concentrations were only saved at the monthly resolution and thus must be approximated for calculations at daily resolution.

Our first approximation assumes a homogeneously distributed vertical profile in which all biomass is stored evenly within the greater of the mixed layer or euphotic depth (referred to as the ProfileDepth, $\left.Z_{\text {profile }}=\max \left(M L D, Z_{\text {eu }}\right)\right)$. Under this assumption the surface concentration (also equivalent to the mean concentration) is calculated by dividing the vertical inventory $\left(\Sigma C_{\text {phyto }}\right)$ by $Z_{\text {profile }}$ (see 2.3.3).

This assumption holds well for deep, well mixed, mixed layers that exceed the euphotic depth [Behrenfeld et al. 2013; Boss and Behrenfeld 2010; Boss et al. 2008]. However the surface concentrations of shallower profiles, where phytoplankton growth can persist in stratified water below the mixed layer, are not approximated as well. In order to improve our approximations, we weight the surface concentration of shallow profiles $\left(Z_{\text {profile }}<100 \mathrm{~m}\right)$ by a spatially and depth dependent correction factor.

\section{A.3 Correction factor model}

We use explicitly resolved monthly surface concentrations to create a model to approximate the true correction factor as a function of grid cell and $Z_{\text {profile }}$.

To do so, we first approximate the monthly surface concentration using the first order approximation defined above and $\Sigma C_{\text {phyto }}$ and $Z_{\text {profile values calculated as described in Chapter }}$ 2.2.3 but from monthly means,

$$
\left[C_{\text {phyto }}\right]_{\text {surf approx }}=\frac{\Sigma C_{\text {phyto }}}{Z_{\text {profile }}} .
$$

We then divide the true surface concentration by the approximated to calculate the correction factor, Cor $_{\text {factor }}$

$$
\text { Cor factor }_{\text {f }}=\frac{\left[C_{\text {phyto }}\right]_{\text {surf true }}}{\left[\begin{array}{c}
{\left[C_{\text {phyto }}\right]_{\text {surf approx }}} \\
166
\end{array}\right.}
$$


Distributions of Cor $_{\text {factor }}$ as a function of $Z_{\text {profile }}$ at each model grid point are then fit to a simple model, such that we can model the requisite $C_{\text {or }}$ factor of the daily data given a grid cell and $Z_{\text {profile }}$.

Figure A-1 shows a typical distribution in which Cor $_{\text {factor }}$ approaches 1.0 as the $Z_{\text {profile }}$ reaches $100 \mathrm{~m}$ (See Figure A-1). Our simple model fits a linear regression to all points where $Z_{\text {profile }}<100 \mathrm{~m}$ and assigns a Cor factor of 1.0 where $Z_{\text {profile }}>100 \mathrm{~m}$ where our uniform distribution assumption is acceptable.

An independent function is modeled at each grid point and compiled. With this model, we can now assign a reasonably well-estimated Cor $_{\text {factor }}$ for any particular spatial coordinate and $Z_{\text {profile }}$ at the daily resolution.

\section{A.4 Test of model skill}

We use monthly mean values of $\Sigma C_{\text {phyto }}$ and $Z_{\text {profile }}$ and our correction factor model to compute corrected approximate surface concentrations that can be directly compared to the true surface concentrations stored at the monthly resolution to test model skill.

In Fig. A-2, plotting time series from one year at the same grid point modeled in Fig. A-1, it is clear the corrected values match the true values much better than our initial approximation (Eq. A.1).

We test the skill of the approximated values at each grid cell. Fig. A-3a projects the mean absolute value percent deviation of the corrected approximation from the true surface values. Error ranges from 5-15\%. More importantly, figure Fig. A-3b shows consistently high $(r .95)$ correlations between the two time series across the Southern Ocean. Thus despite minor discrepancies in the absolute magnitude, seasonal trends (our primary concern) remain tightly coupled. Table A.1 outlines the percent deviation and correlation coefficient values spatially averaged over each swath considered in 2.3.2 \& 2.3.3 in addition to the entire Southern Hemisphere.

\begin{tabular}{|c|c|c|c|c|c|}
\hline & P1 & P2 & P3 & A1 & SH \\
\hline Mean \% Deviation & 3.83 & 6.53 & 11.75 & 12.04 & 10.98 \\
\hline Correlation Coefficient & .984 & .983 & .987 & .988 & .82 \\
\hline
\end{tabular}

Table A.1: The percent deviation and correlation coefficient values spatially averaged over each swath considered in 2.3.2 \& 2.3.3 in addition to the entire Southern Hemisphere (SH). 


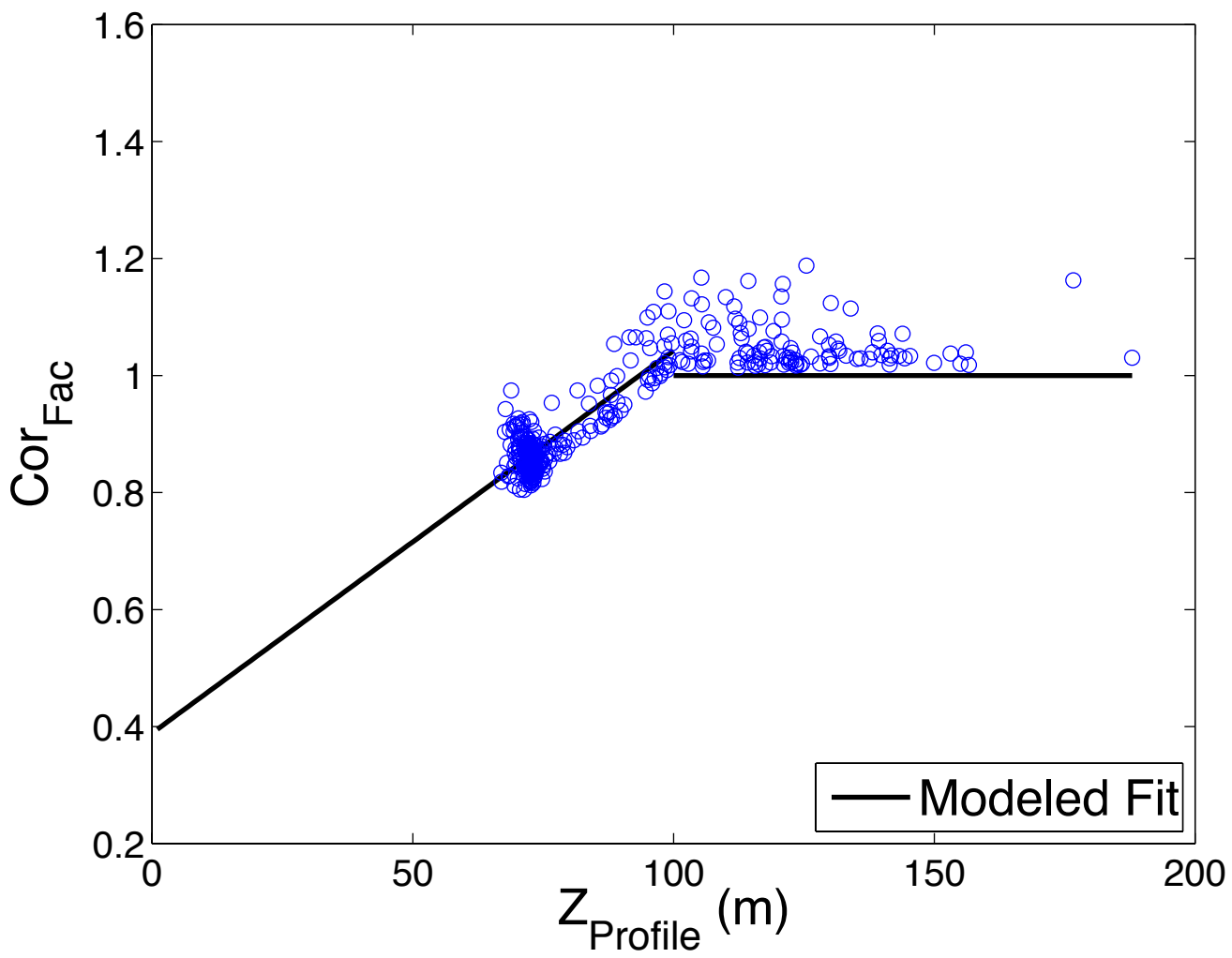

Figure A-1: The Cor $_{\text {Factor }}$ needed to convert the approximated surface concentration to the true surface concentration plotted as a function of $Z_{\text {profile }}$ for the timeseries at a single grid point (Lat $=$ $50^{\circ} \mathrm{S}$, Lon $=91^{\circ} \mathrm{W}$ ) and monthly resolution. The modeled fit is plotted in black.

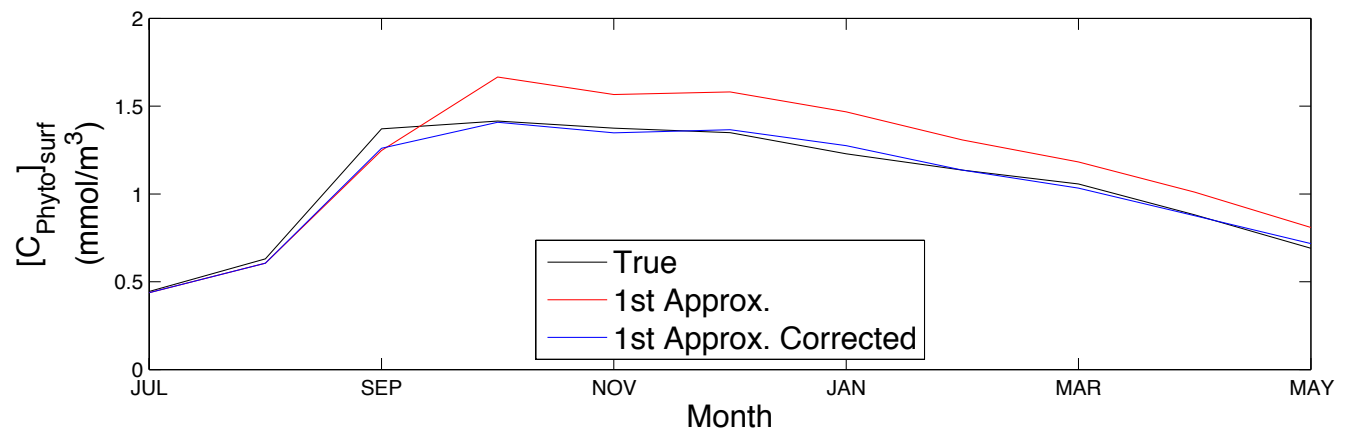

Figure A-2: A single year, monthly resolution time series plotted for the true surface phytoplankton biomass concentration (black trace) along a first approximation (red trace) and the corrected approximation (blue trace). Time series are from the same grid cell as Fig. A1 (Lat $=50^{\circ} \mathrm{S}$, Lon $=91^{\circ}$ $\mathrm{W})$. 


\section{Mean absolute \% deviation b/w True and Corrected $\left[\mathbf{C}_{\text {Phyto }}\right]_{\text {surf }}$}

a.

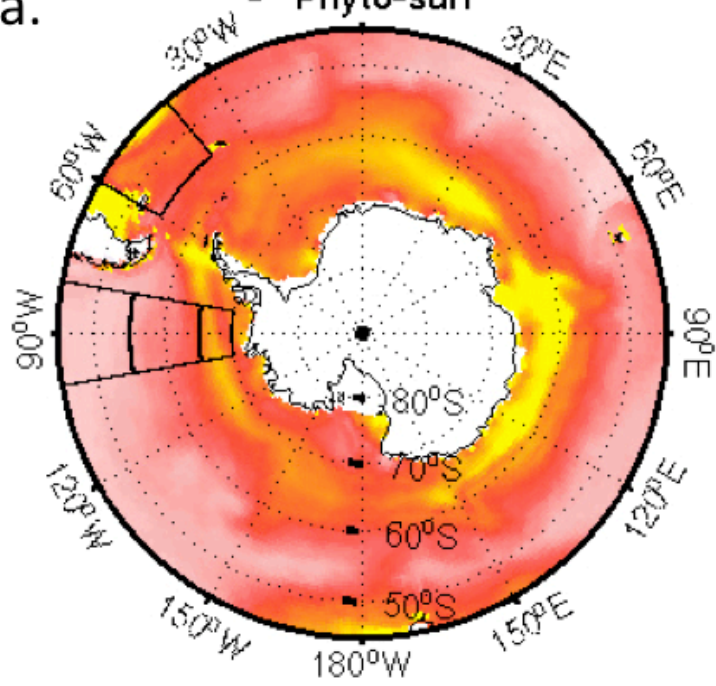

\section{Correlation between} True and Corrected

$\%$

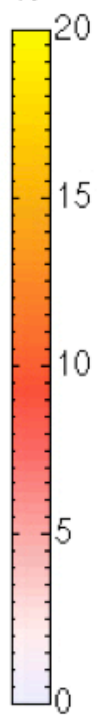

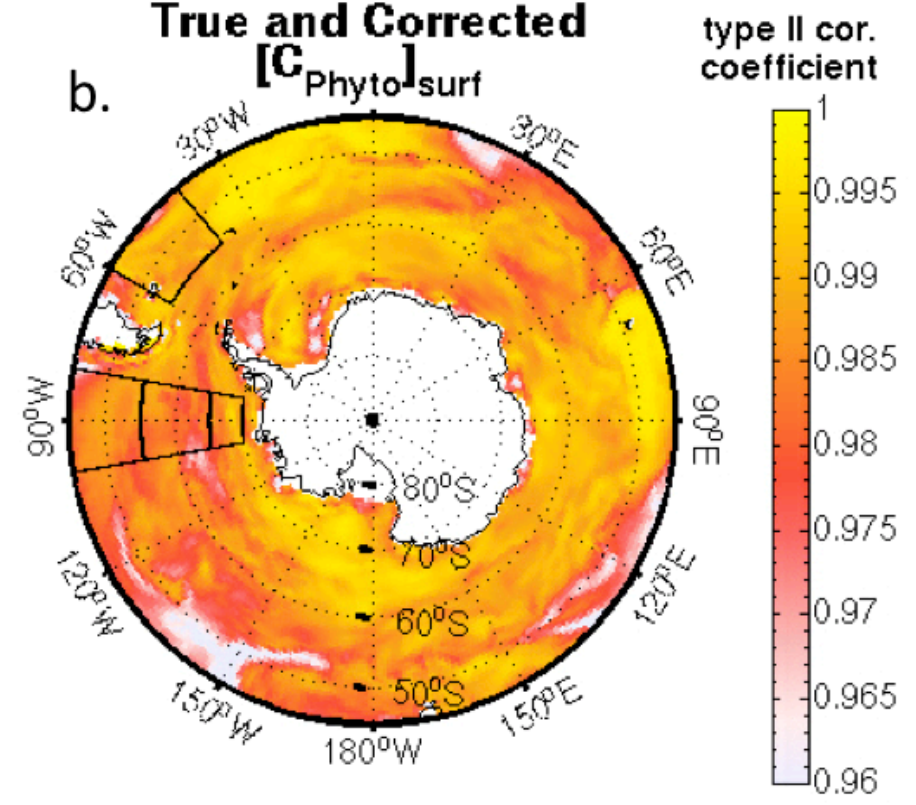

Figure A-3: (a) The mean absolute value percent deviation of the corrected approximation from the true surface values for each grid cell is plotted on the left and (b) the correlation coefficient between the two time series plotted on the right. 
ApPendix B

\section{SUPPLEMENTAL SEASONAL DISTRIBUTIONS OF CLIMATOLOGIES AND EDDY ANOMALIES FROM Chapter 3}



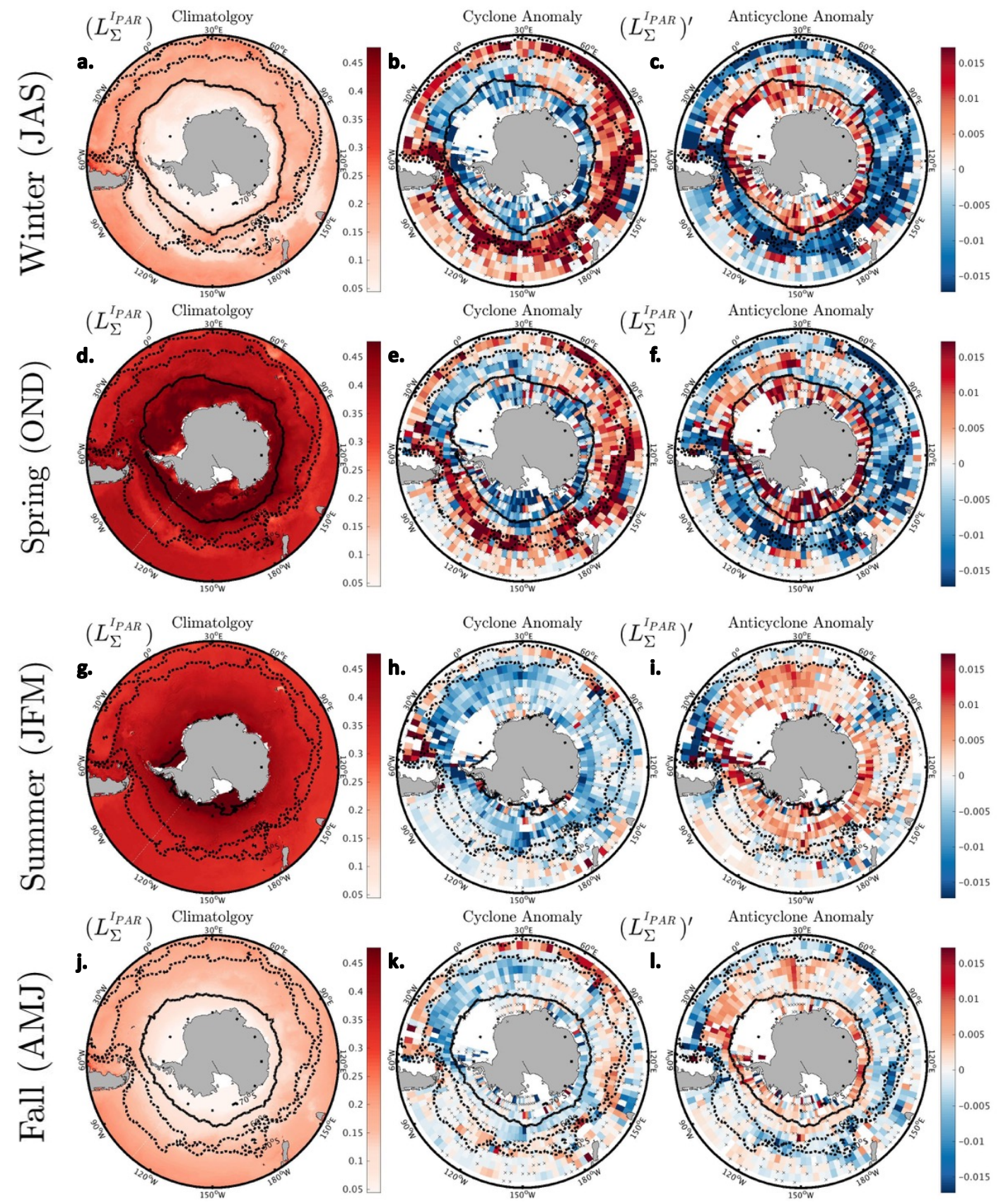

Figure B-1: Seasonal $L_{\Sigma}^{I_{P A R}}$ climatology and eddy anomalies. $(\mathbf{a}, \mathbf{d}, \mathbf{g}, \mathbf{j})$ The simulated climatologic $L_{\Sigma}^{I_{P A R}}$. $(\mathbf{b}, \mathbf{e}, \mathbf{h}, \mathbf{k})$ The simulated mean $L_{\Sigma}^{I_{P A R^{\prime}}}$ in all cyclones. $(\mathbf{c}, \mathbf{f}, \mathbf{i}, \mathbf{l})$ The simulated mean $L_{\Sigma}^{I_{P A R}}$ in all anticyclones. Data is seasonally binned as denoted in each row. Eddy realizations are further averaged over $3^{\circ} \mathrm{x} 3^{\circ}$ spatial bins. Each bin may contain multiple realizations from the same eddy track provided that each time step falls within the seasonal threshold. Bins that are not statistically different from 0 at the $95 \%$ confidence level are denoted with an ' $x$ '. The $10 \%$ ice contour is denoted in black. The ACC $\left(-80 \mathrm{~cm}<\overline{S S H}_{C l i m}<-20 \mathrm{~cm}\right)$ is denoted in with a dashed black line. 

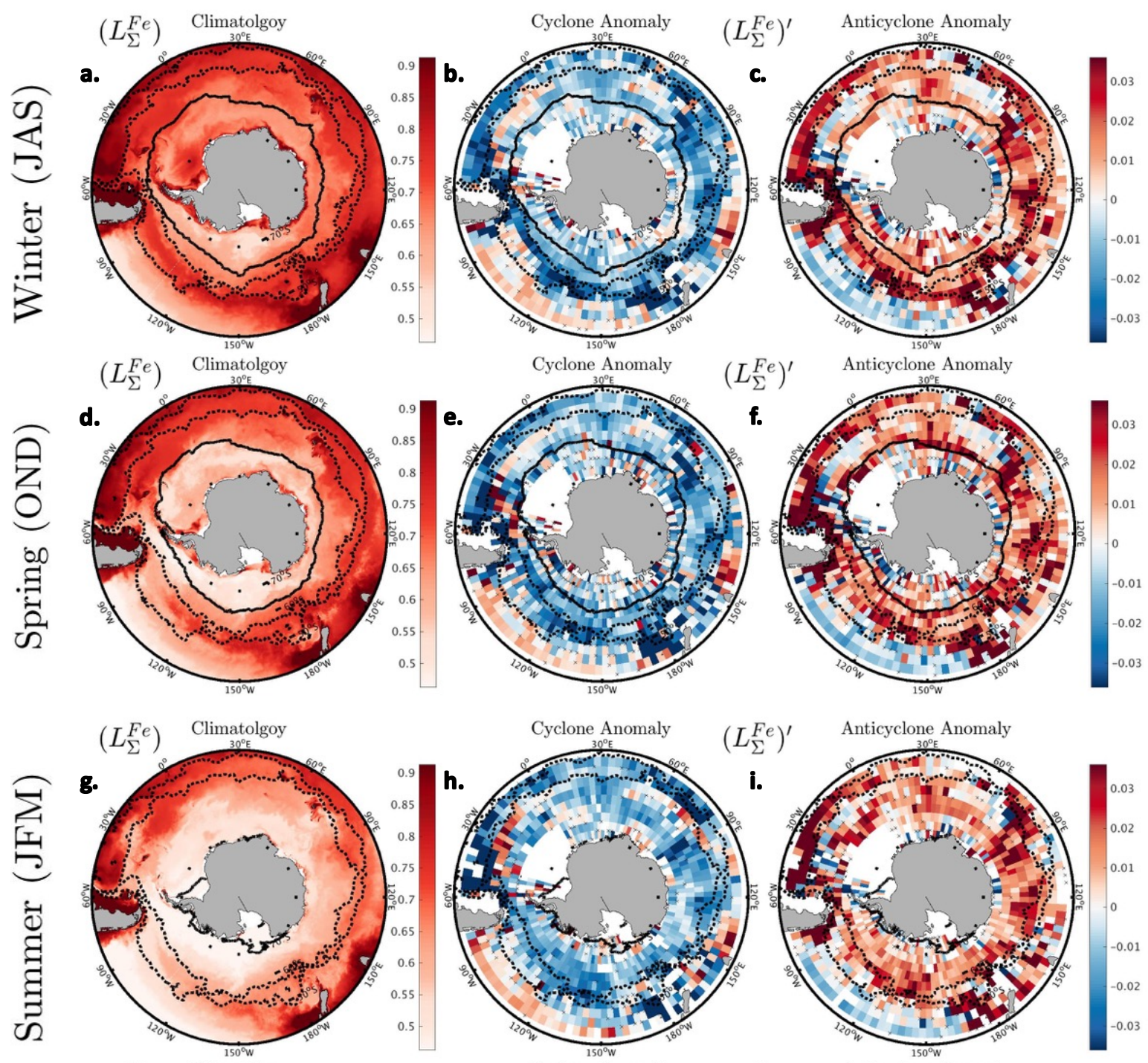

$\left(L_{\Sigma}^{F e}\right)^{\prime}$
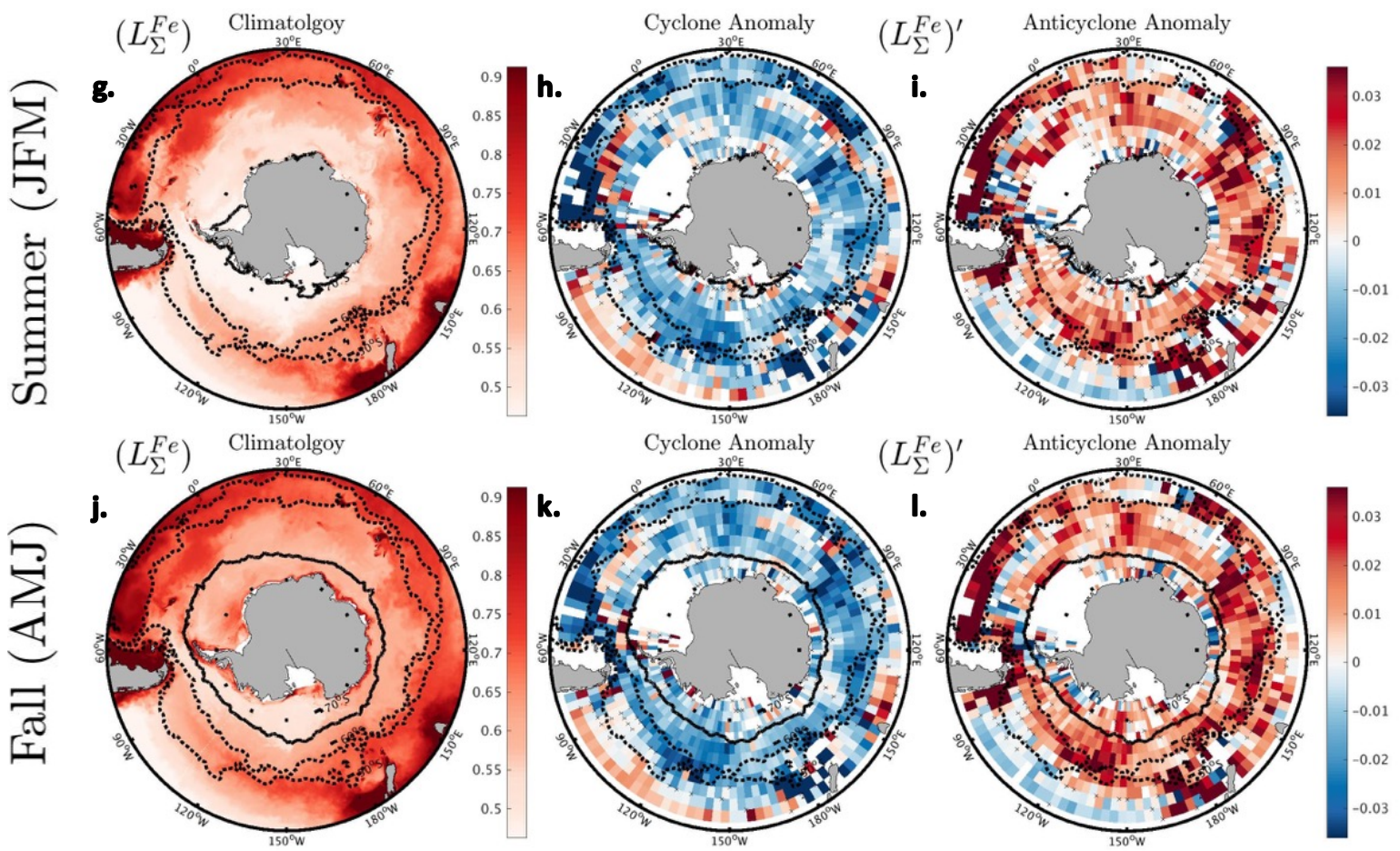

Figure B-2: Seasonal $L_{\Sigma}^{F e}$ climatology and eddy anomalies. Same as Fig. B-1 except for $L_{\Sigma}^{F e}$. 

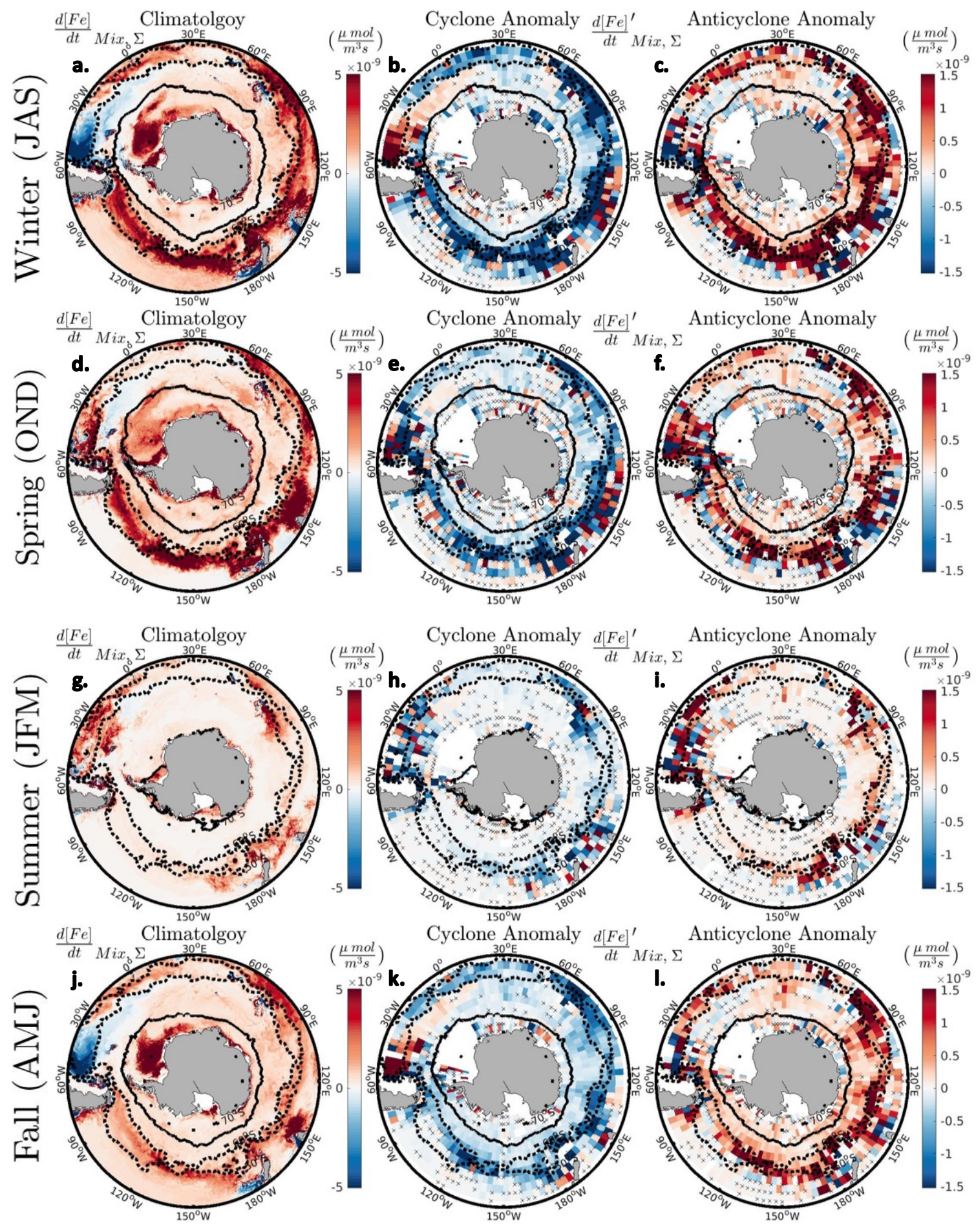

Figure B-3: Seasonal $\frac{d[F e]}{d t}_{M i x, \Sigma}$ climatology and eddy anomalies. Same as Fig. B-1 except for $\frac{d[F e]}{d t}{ }_{M i x, \Sigma}$. 

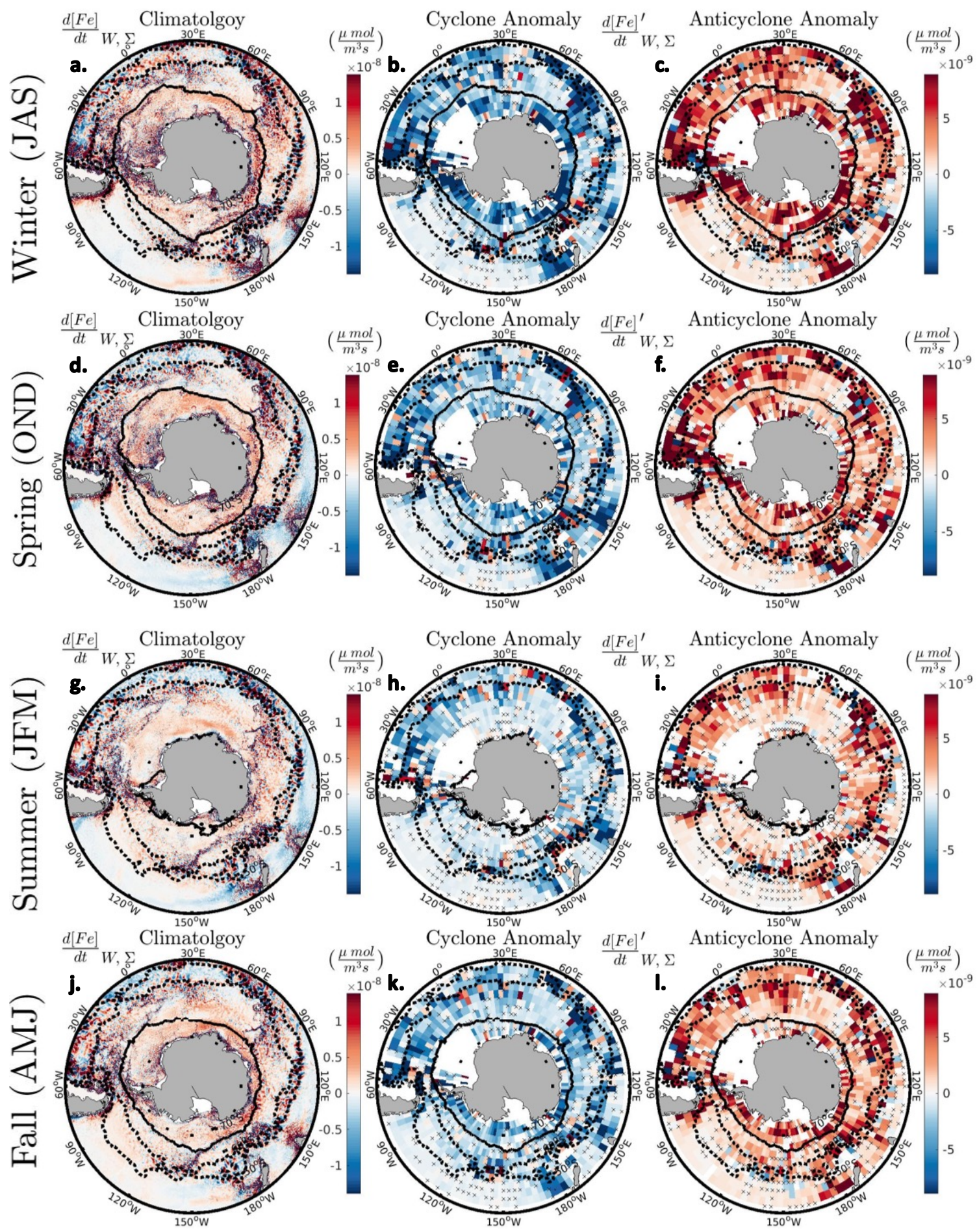

Figure B-4: Seasonal $\frac{d[F e]}{d t}_{W, \Sigma}$ climatology and eddy anomalies. Same as Fig. B-1 except for $\frac{d[\mathrm{Fe}]}{d t}_{W, \Sigma}$. 

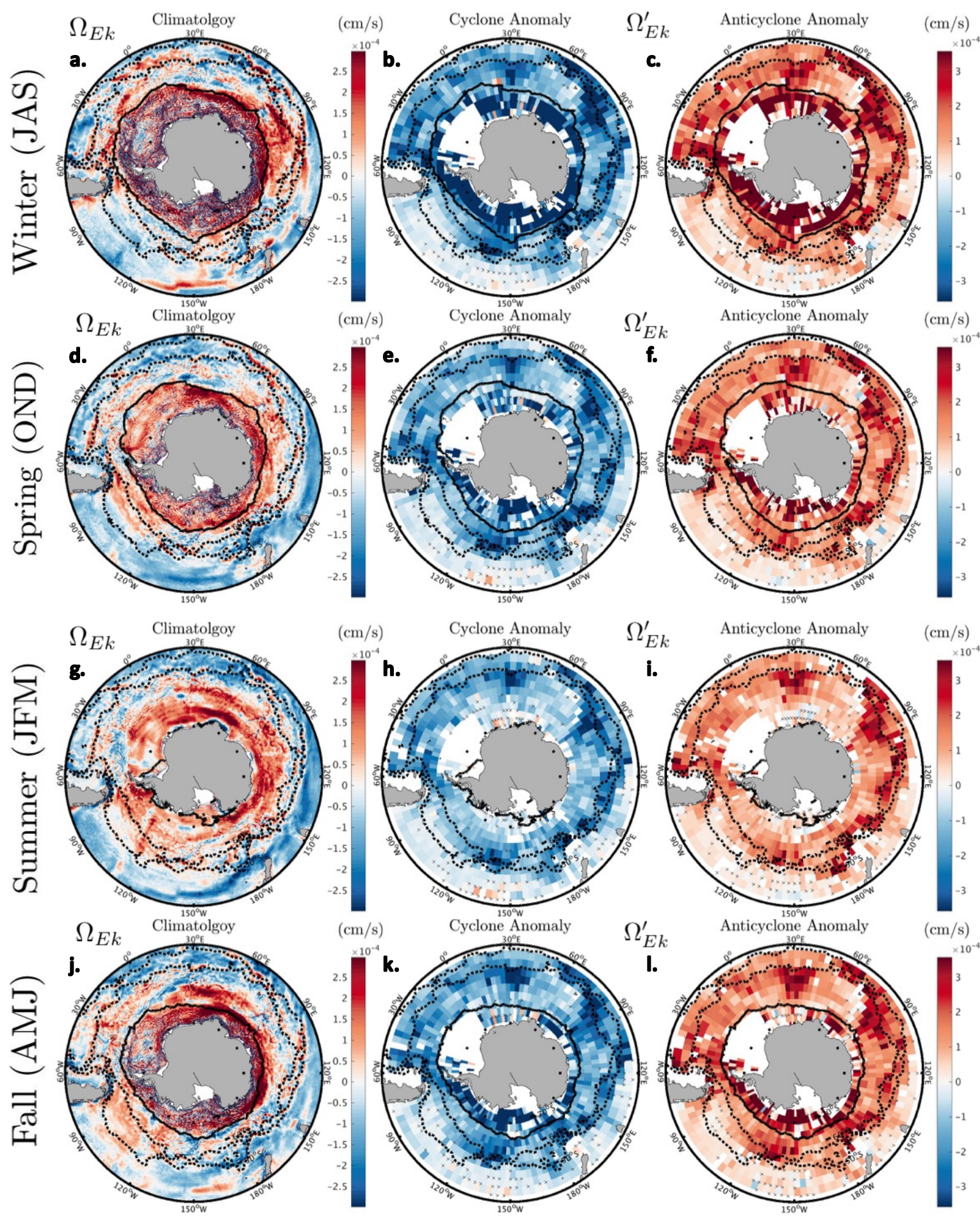

Figure B-5: Seasonal $\Omega_{E k}$ climatology and eddy anomalies. Same as Fig. B-1 except for $\Omega_{E k}$. 
Appendix C

\section{SUPPLEMENTAL SEASONAL DISTRIBUTIONS OF CLIMATOLOGIES AND EDDY ANOMALIES FROM \\ CHAPTER 4}



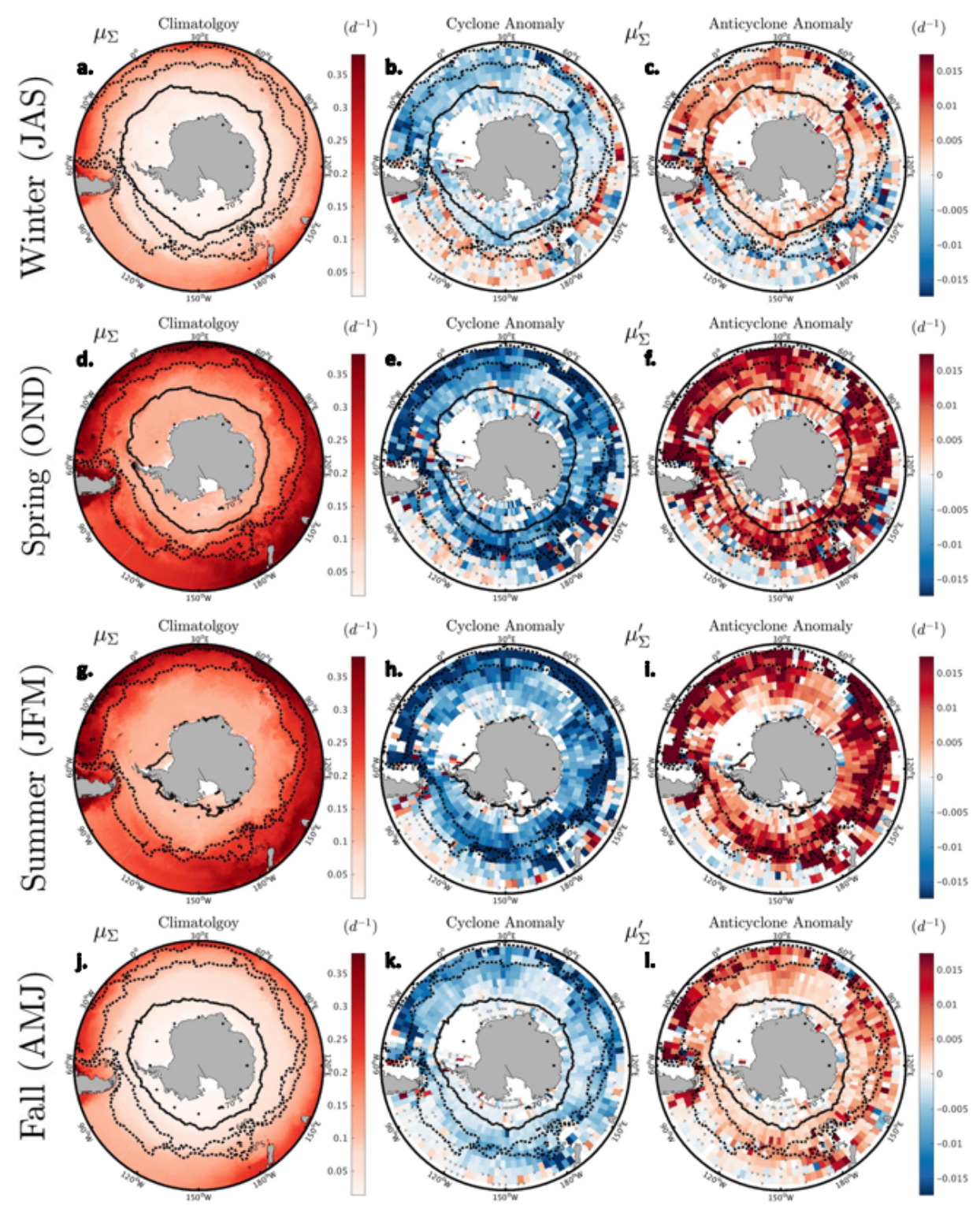

Figure C-1: Seasonal $\mu_{\Sigma}$ climatology and eddy anomalies. (a, d, g, j) The simulated climatologic $\mu_{\Sigma}$. (b, e, h, k) The simulated mean $\mu_{\Sigma}^{\prime}$ in all cyclones. $(\mathbf{c}, \mathbf{f}, \mathbf{i}, \mathbf{l})$ The simulated mean $\mu_{\Sigma}^{\prime}$ in all anticyclones. Data is seasonally binned as denoted in each row. Eddy realizations are further averaged over $3^{\circ} \times 3^{\circ}$ spatial bins. Each bin may contain multiple realizations from the same eddy track provided that each time step falls within the seasonal threshold. Bins that are not statistically different from 0 at the $95 \%$ confidence level are denoted with an ' $x$ '. The $10 \%$ ice contour is denoted in black. The ACC $\left(-80 \mathrm{~cm}<\overline{S S H}_{\text {Clim }}<-20 \mathrm{~cm}\right)$ is denoted in with a dashed black line. 

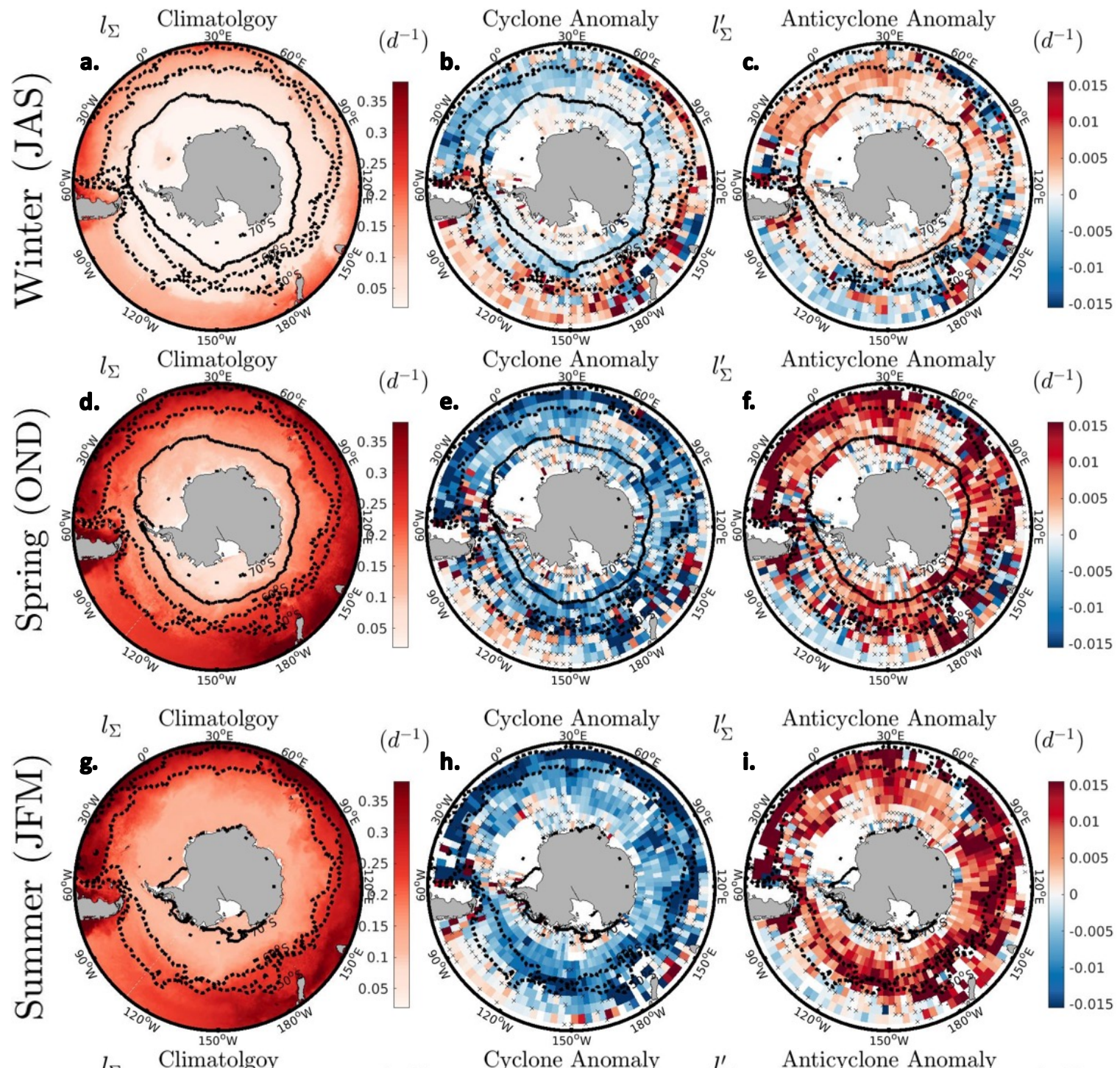

Cyclone Anomaly
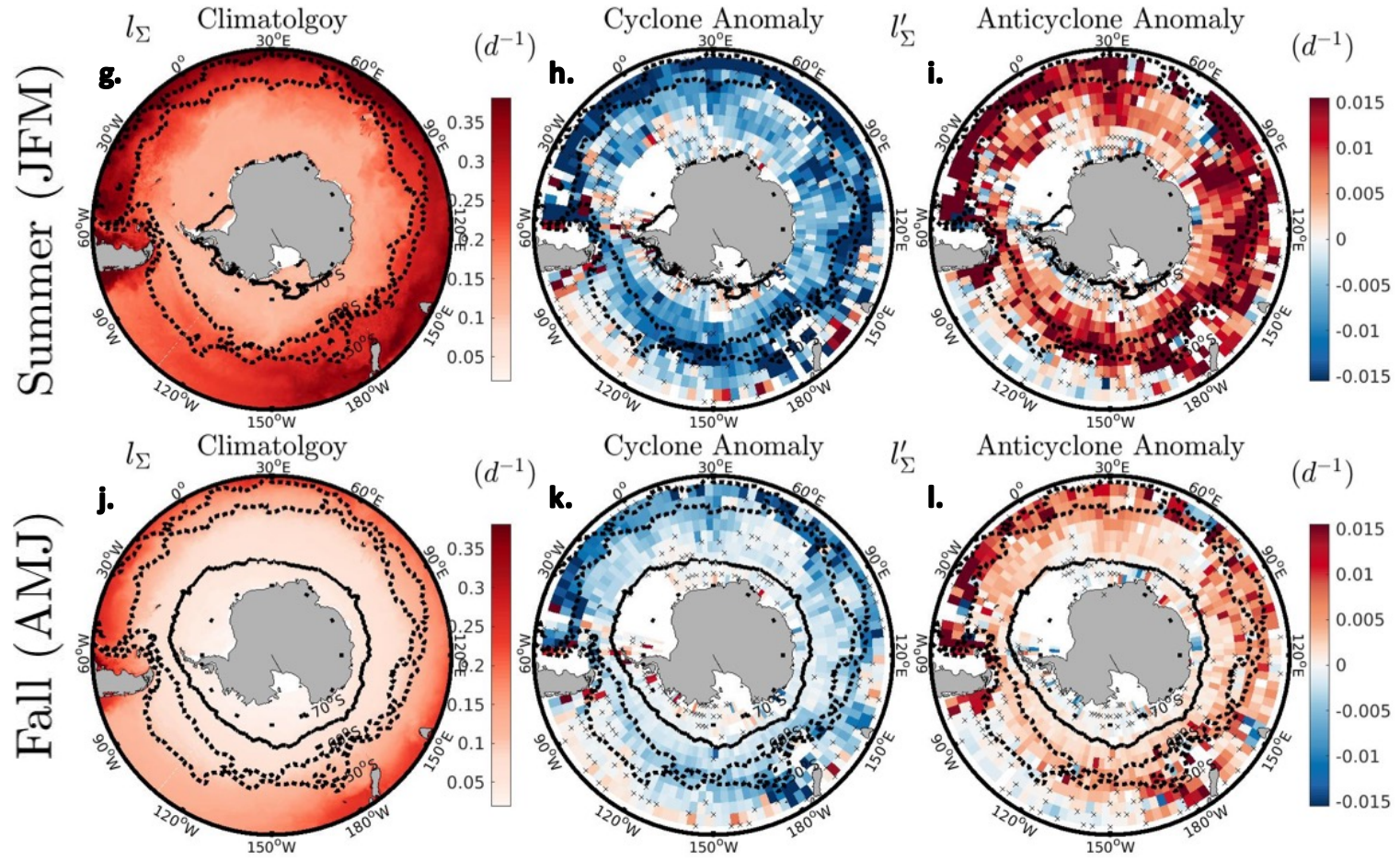

Figure C-2: Seasonal $l_{\Sigma}$ climatology and eddy anomalies. Same as Fig. C-1 except for $l_{\Sigma}$. 

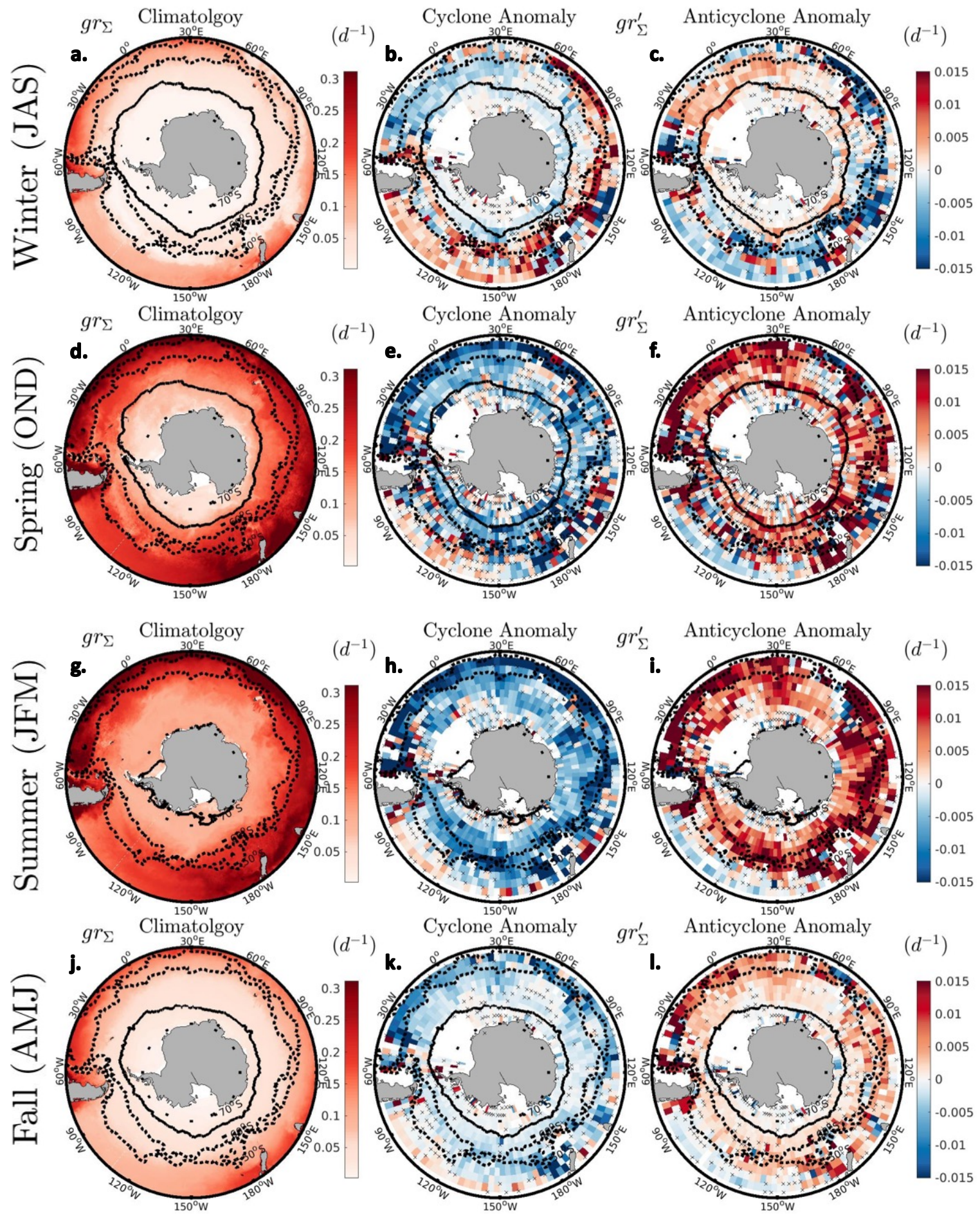

Figure C-3: Seasonal $g r_{\Sigma}$ climatology and eddy anomalies. Same as Fig. C-1 except for $g r_{\Sigma}$. 

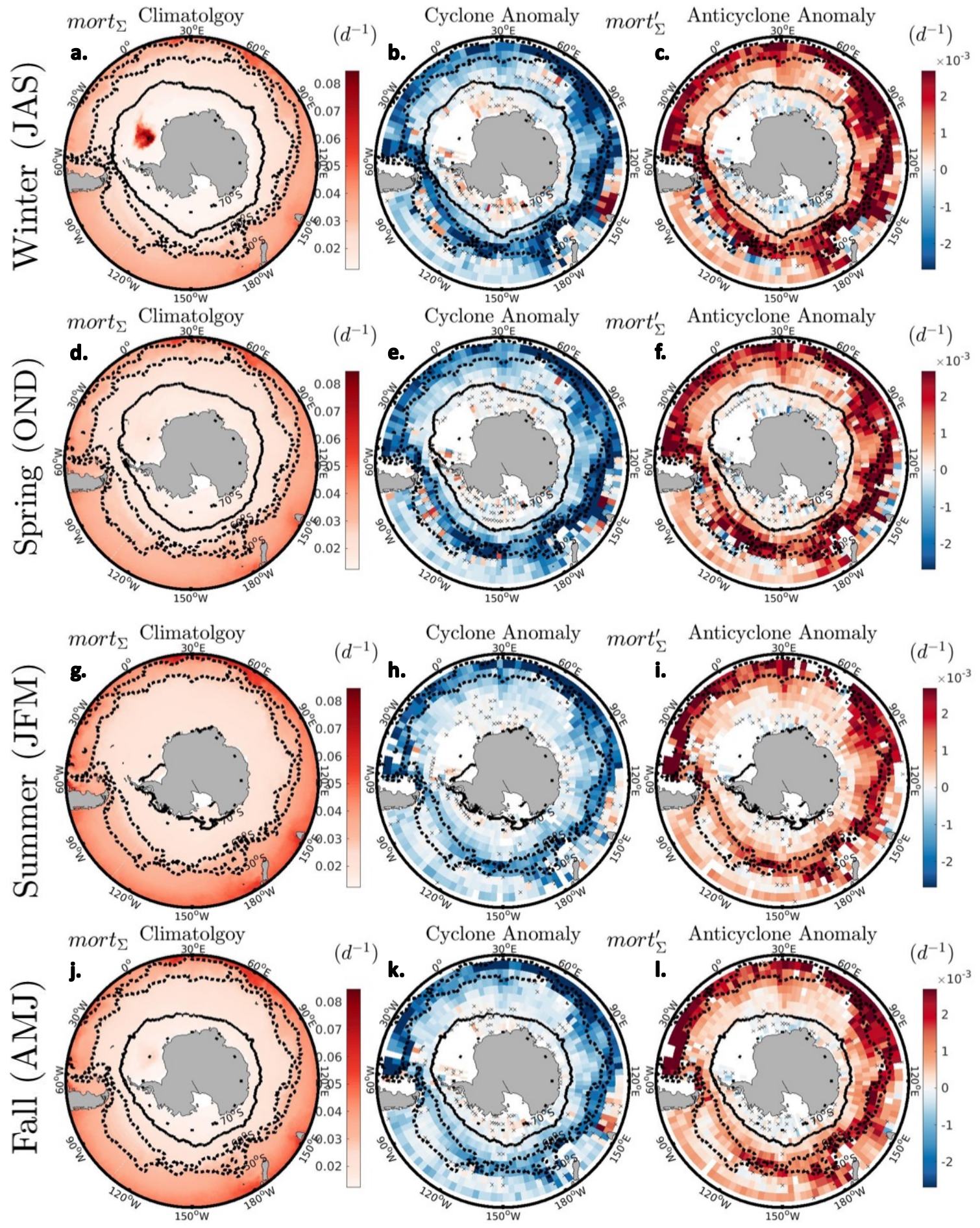

Figure C-4: Seasonal mort $t_{\Sigma}$ climatology and eddy anomalies. Same as Fig. C-1 except for mort $t_{\Sigma}$. 

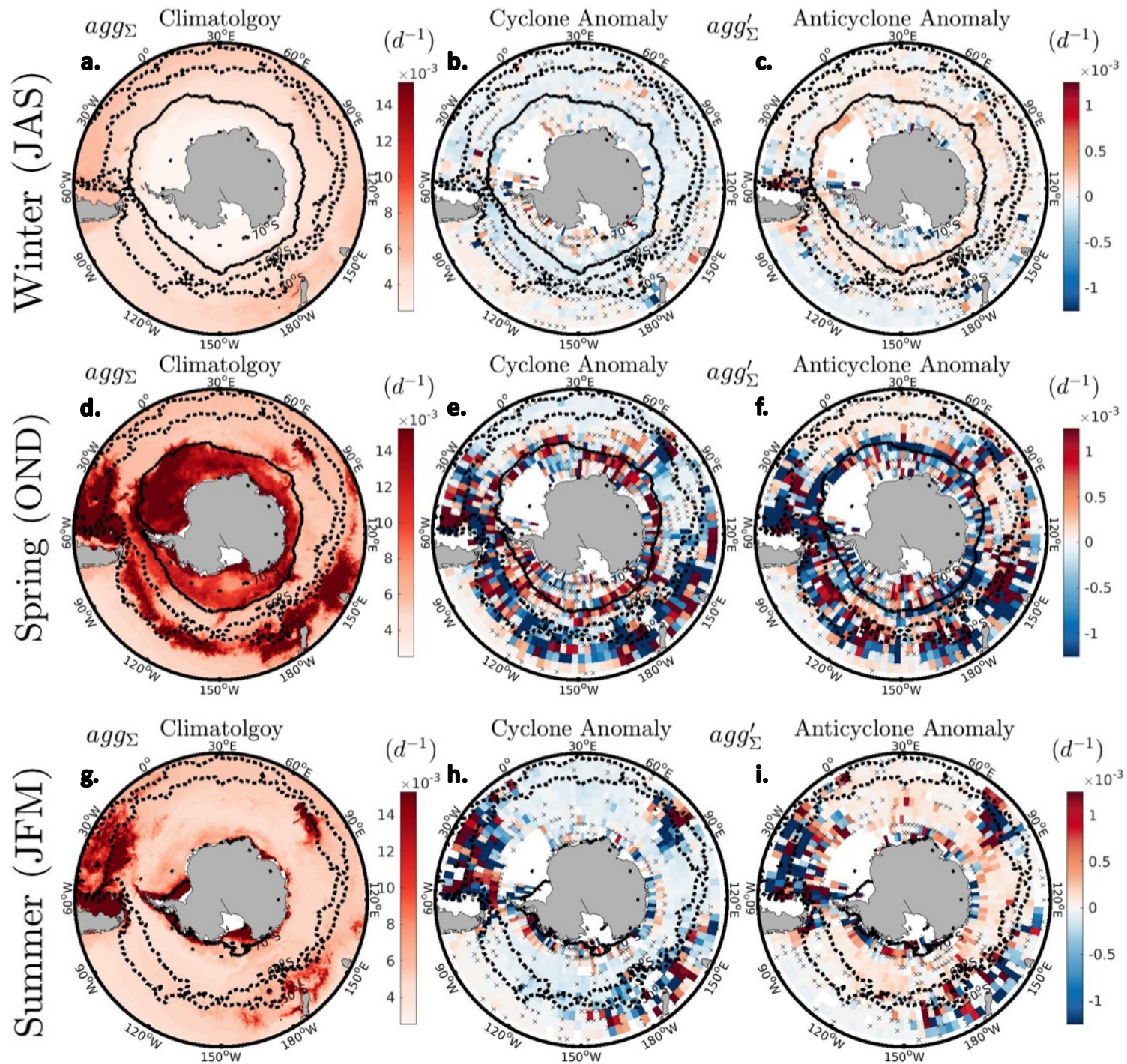

Cyclone Anomaly

$a g g_{\Sigma}^{\prime} \quad$ Anticyclone Anomaly
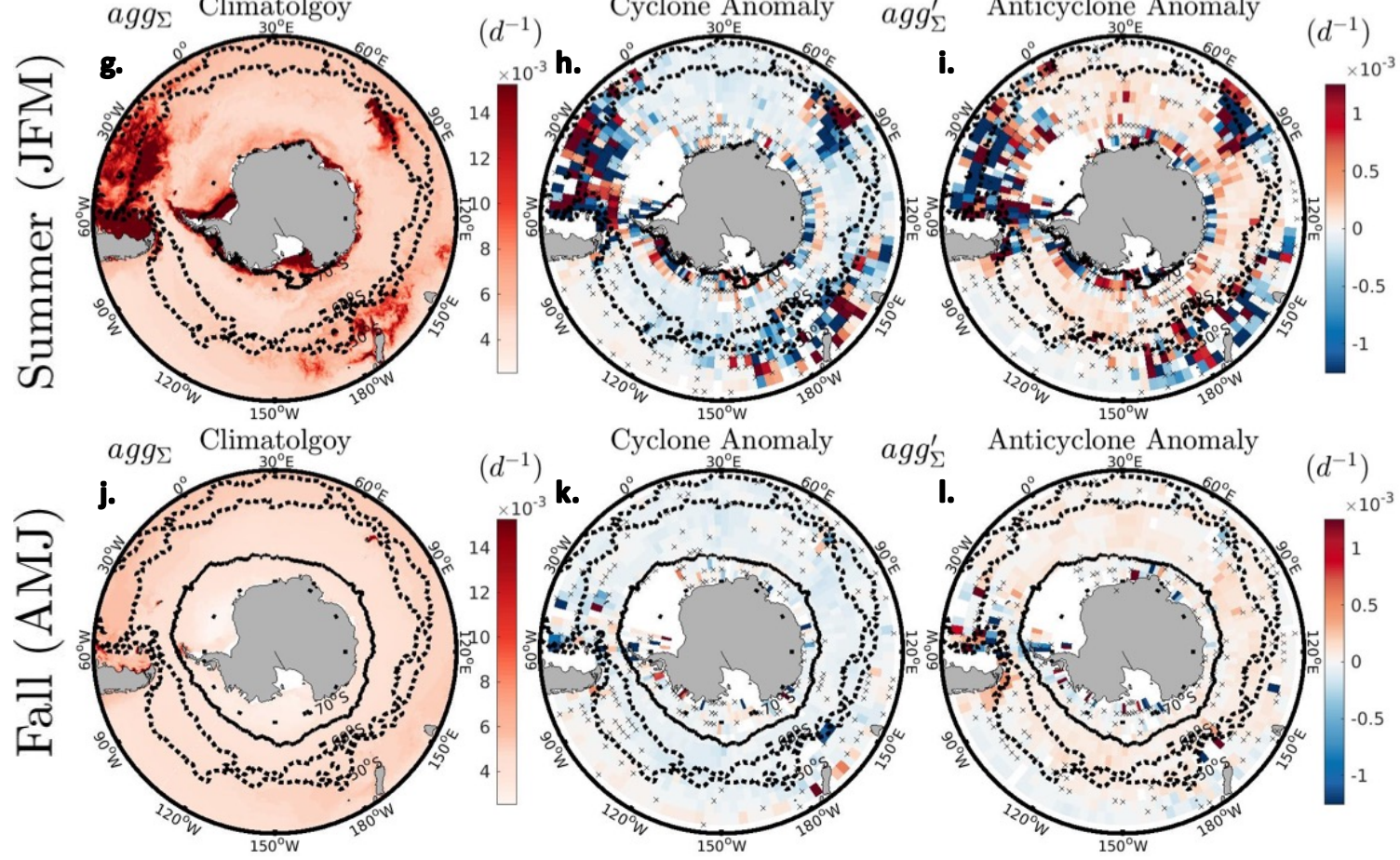

Figure C-5: Seasonal $a g g_{\Sigma}$ climatology and eddy anomalies. Same as Fig. C-1 except for $a g g_{\Sigma}$. 

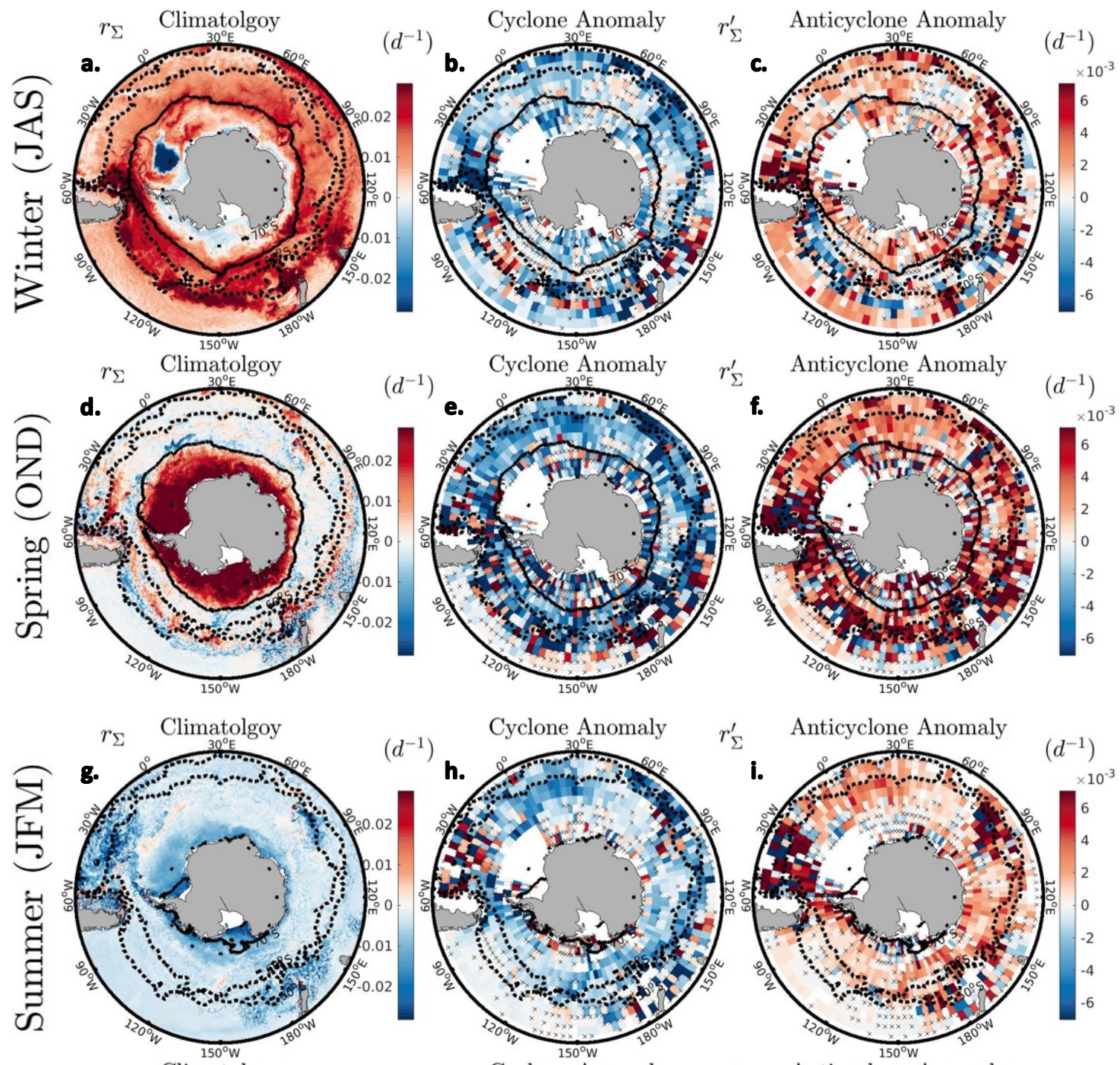

Cyclone Anomaly
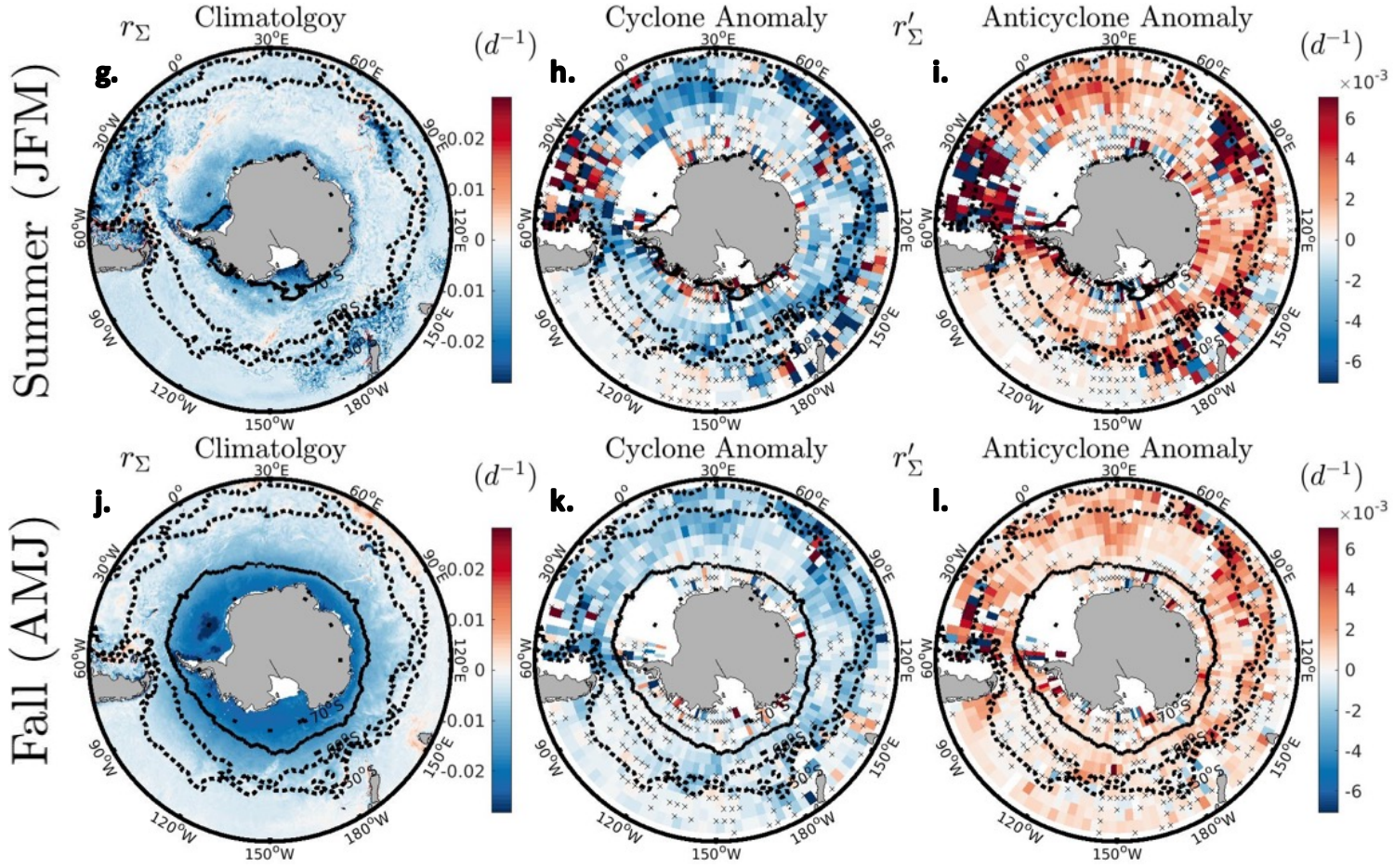

Figure C-6: Seasonal $r_{\Sigma}$ climatology and eddy anomalies. Same as Fig. C-1 except for $r_{\Sigma}$. 

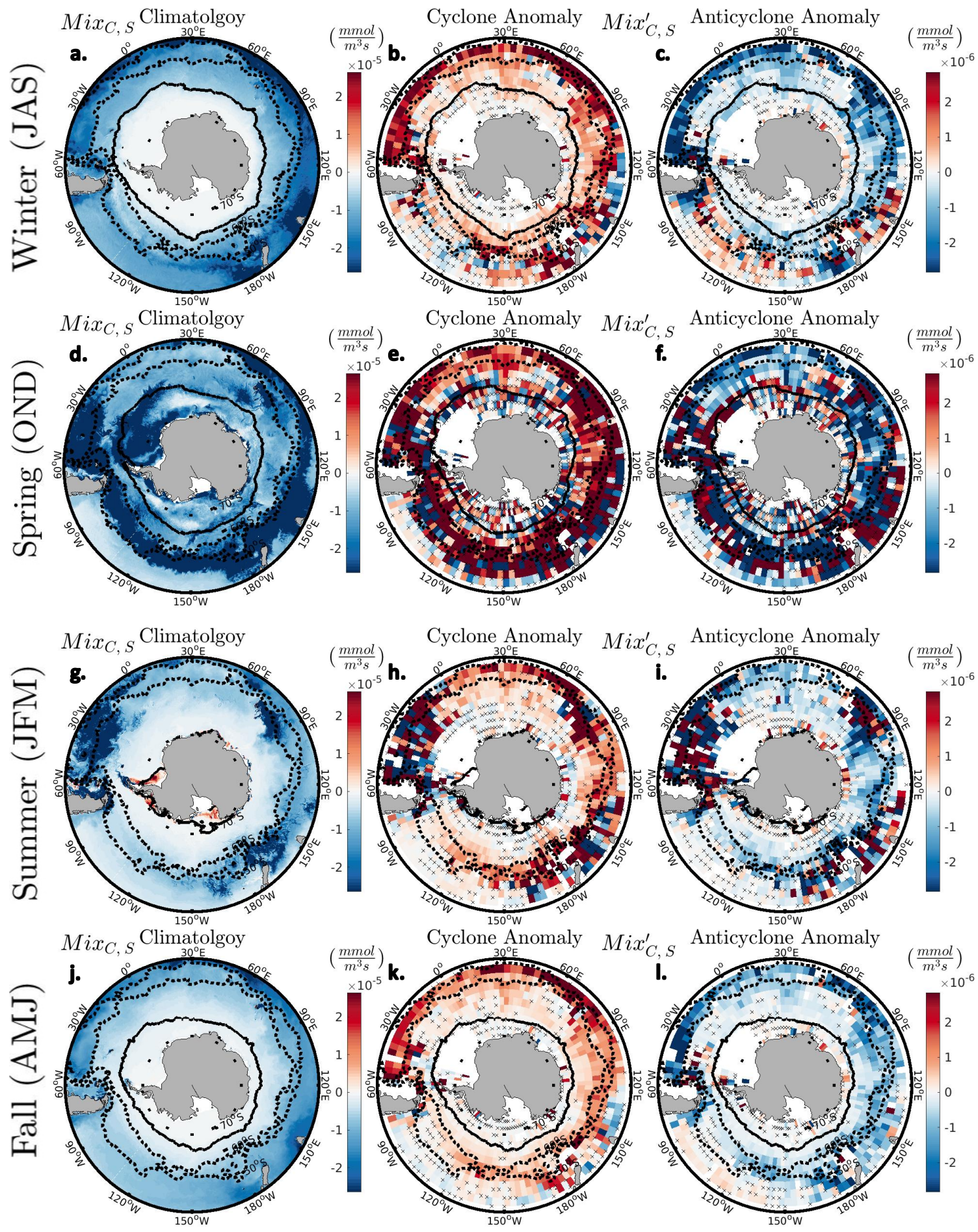

Figure C-7: Seasonal $M i x_{C, S}$ climatology and eddy anomalies. Same as Fig. C-1 except for $M i x_{C, S}$. 

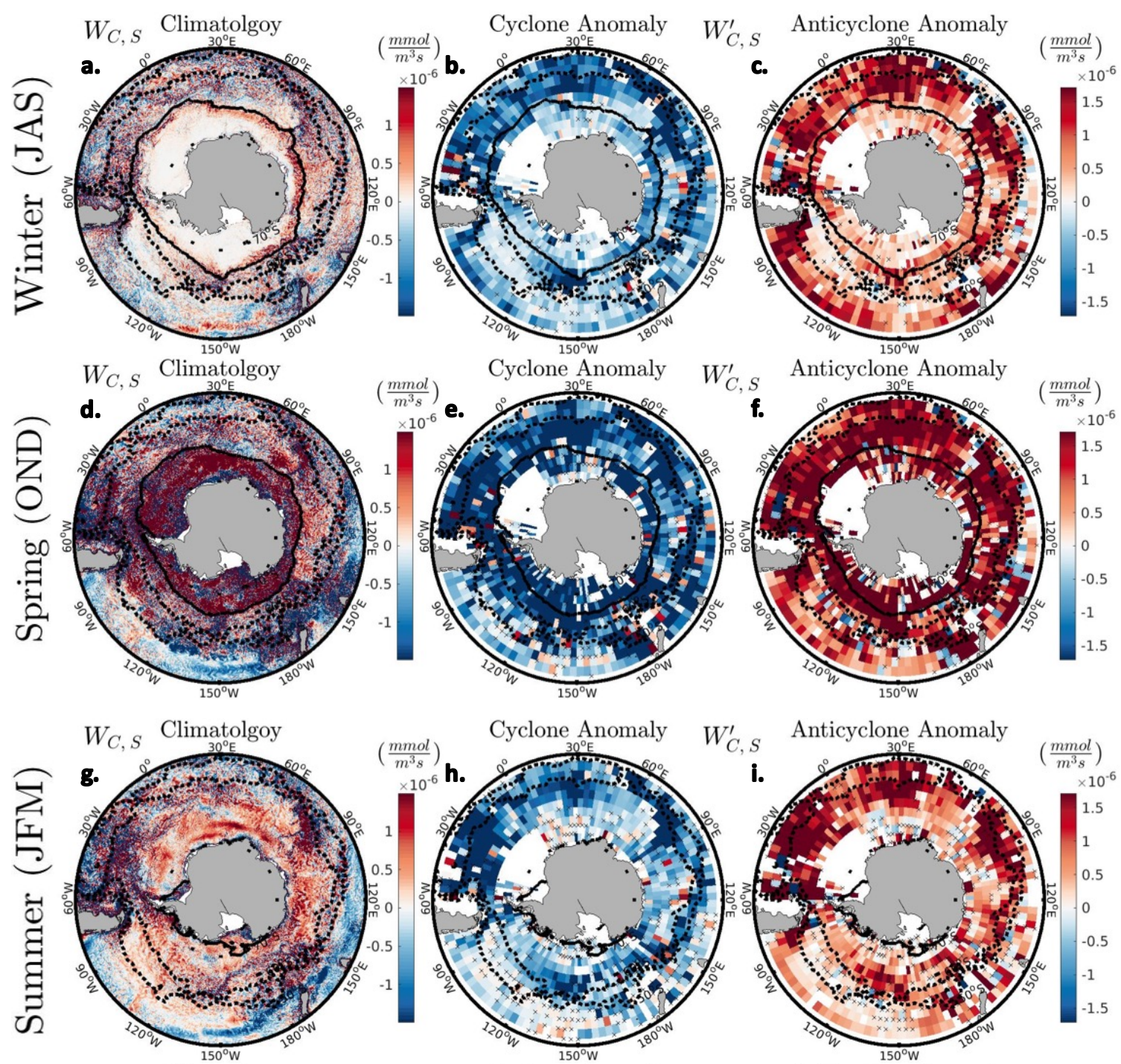

Cyclone Anomaly
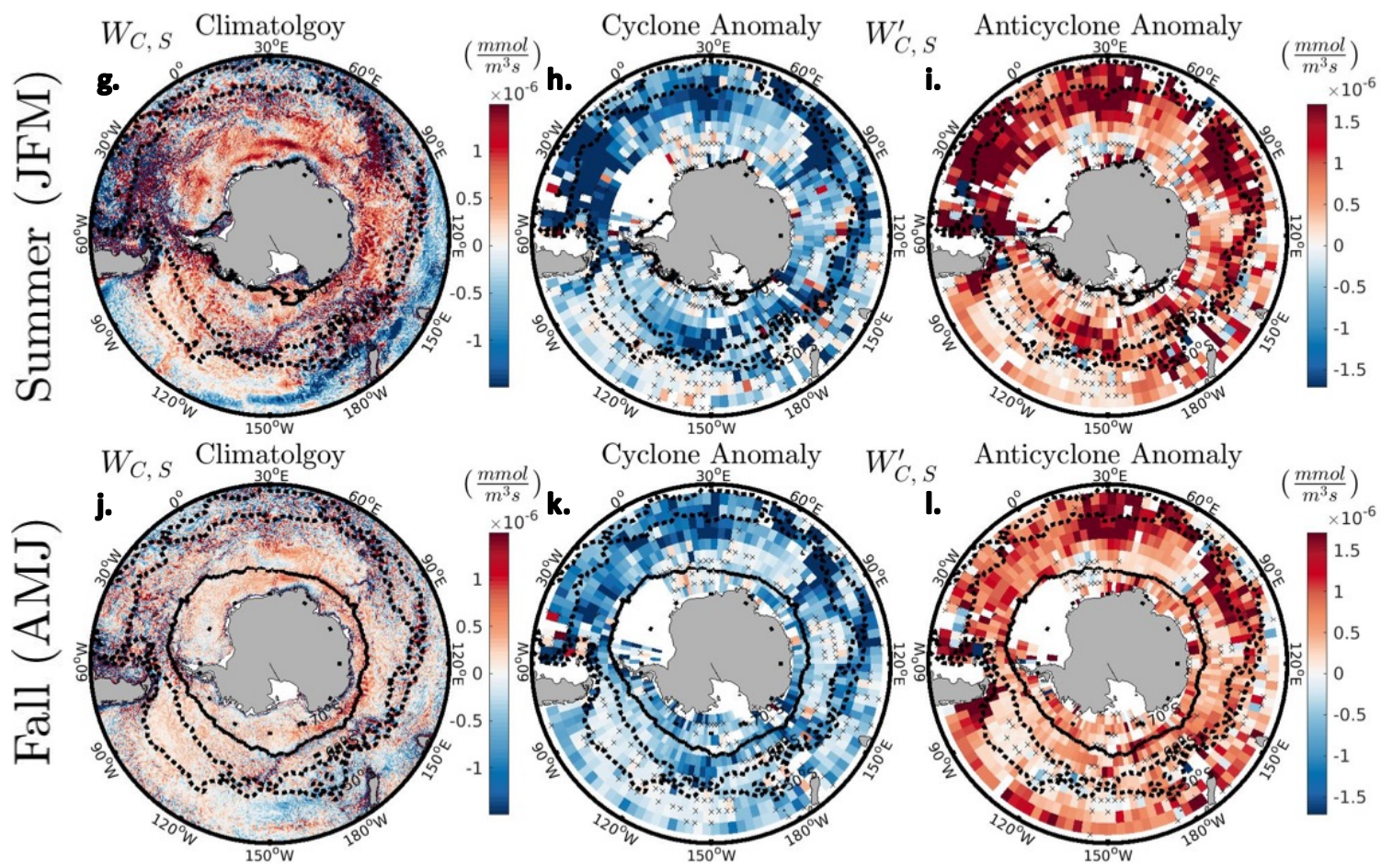

Figure C-8: Seasonal $W_{C, S}$ climatology and eddy anomalies. Same as Fig. C-1 except for $W_{C, S}$. 

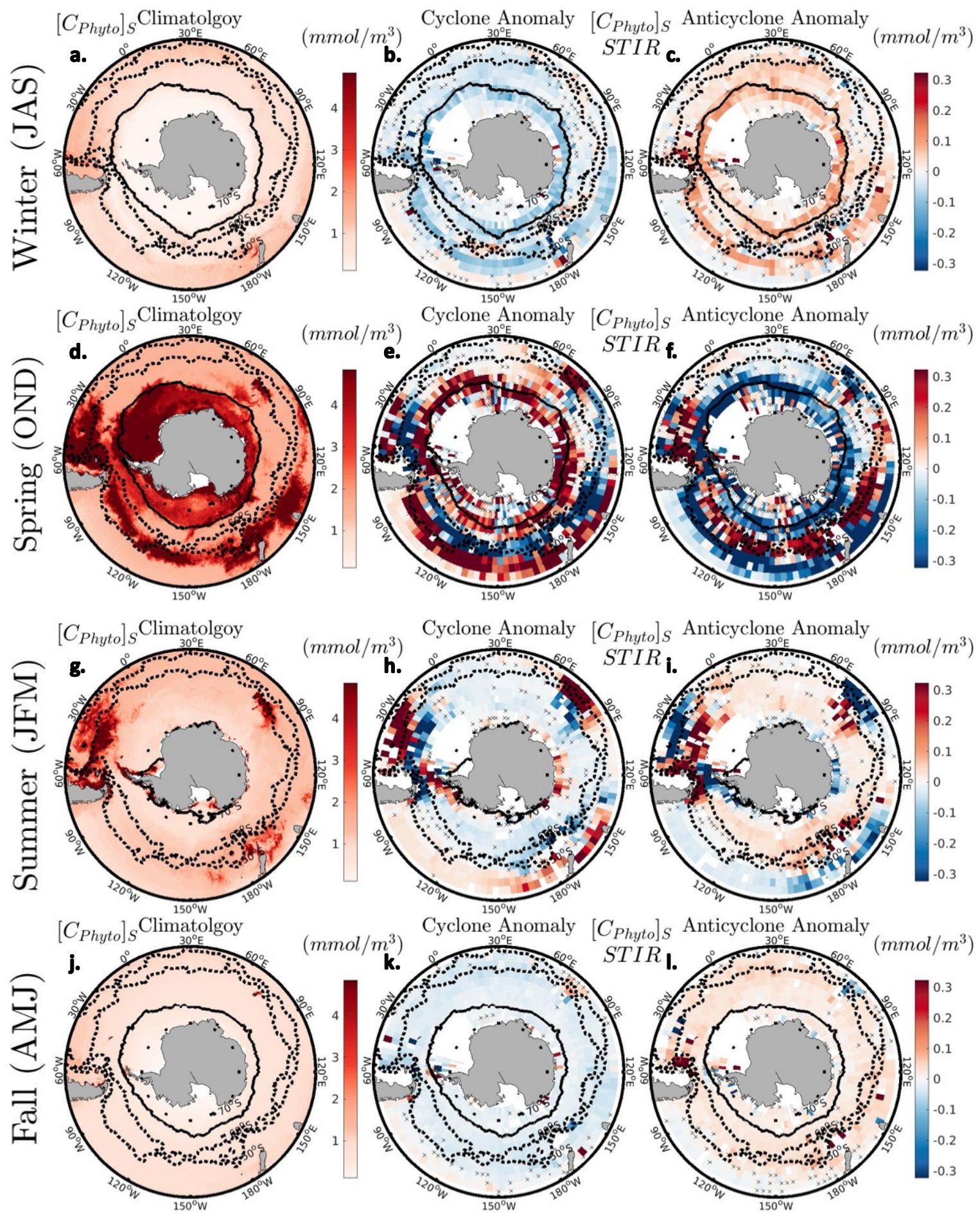

Figure C-9: Seasonal $\left[C_{P h y t o}\right]_{S} S T I R$ climatology and eddy anomalies. Same as Fig. C-1 except for $\left[C_{P h y t o}\right]_{S} S T I R$. 

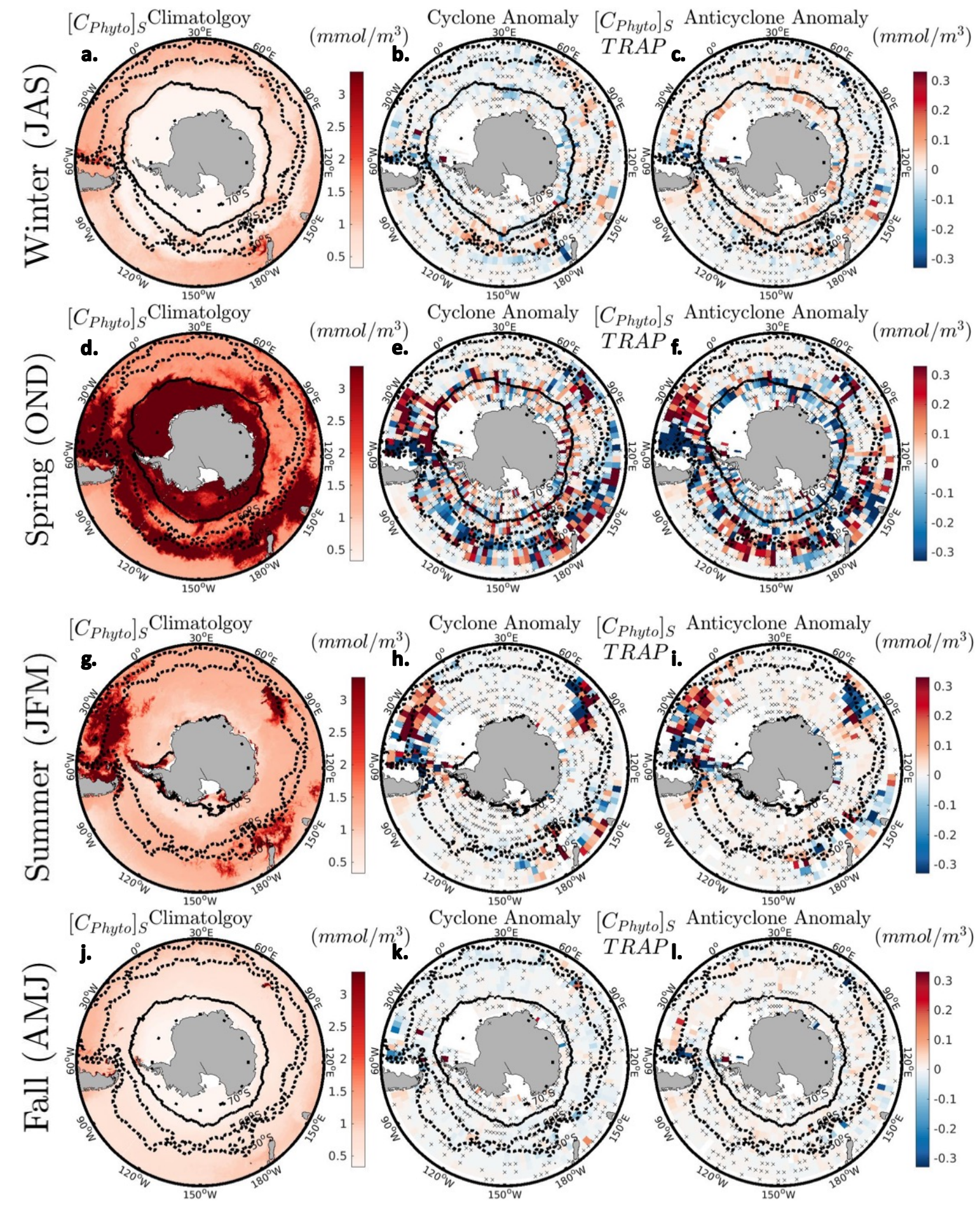

Figure C-10: Seasonal $\left[C_{P h y t o}\right]_{S} T R A P$ climatology and eddy anomalies. Same as Fig. C-1 except for $\left[C_{P h y t o}\right]_{S} T R A P$. 

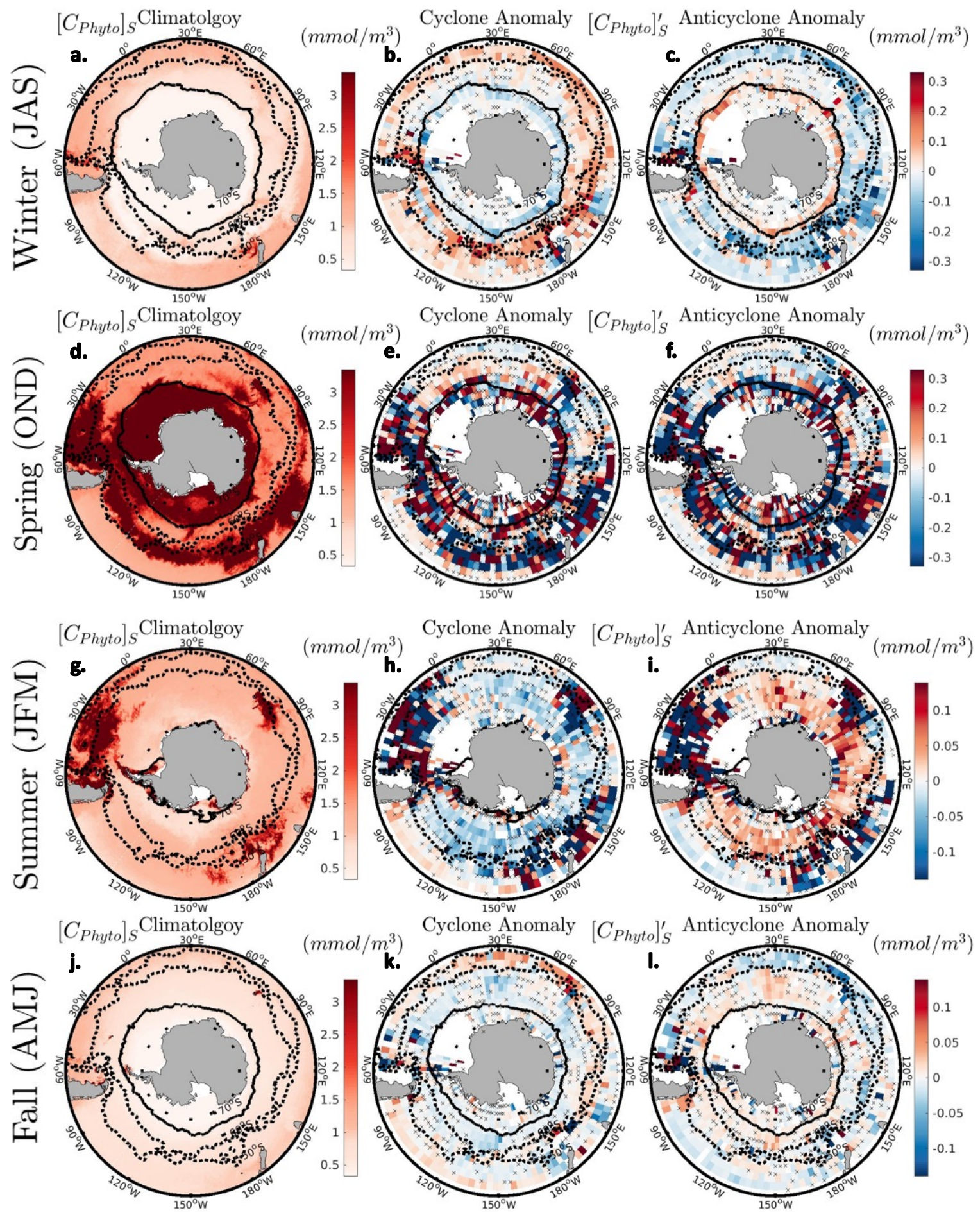

Figure C-11: Seasonal $\left[C_{P h y t o}\right]_{S}$ climatology and eddy anomalies. Same as Fig. C-1 except for $\left[C_{\text {Phyto }}\right]_{S}$. 

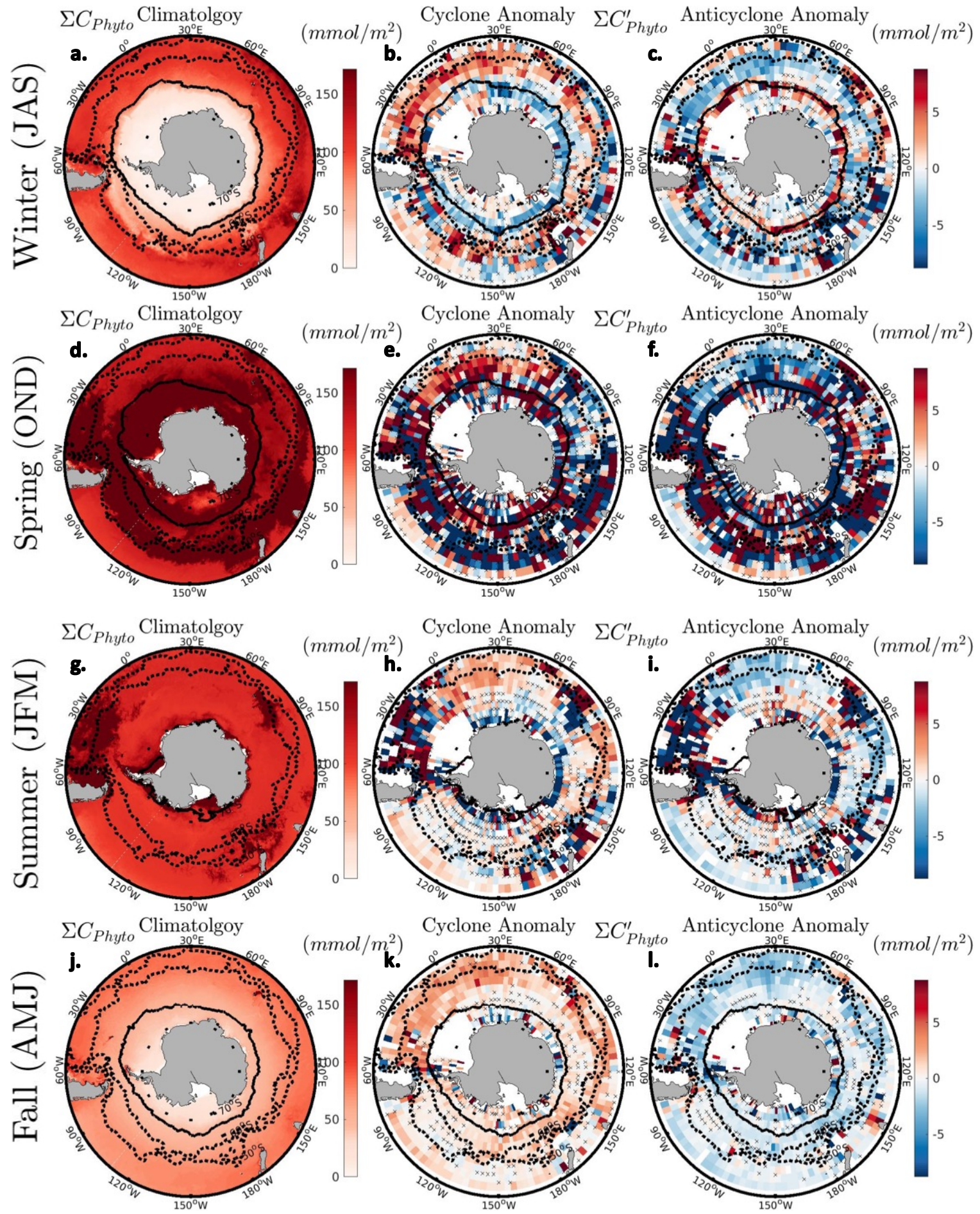

Figure C-12: Seasonal $\Sigma C_{P h y t o}$ climatology and eddy anomalies. Same as Fig. C-1 except for $\Sigma C_{\text {Phyto }}$. 
Appendix D

\section{REFERENCES}




\section{REFERENCES}

Abraham, Edward R. (1998). "The Generation of Plankton Patchiness by Turbulent Stirring". en. In: Nature 391.6667, pp. 577-580. ISSN: 1476-4687. DOI: 10.1038/35361.

Abrahamsen, Einar P. (2014). "Sustaining Observations in the Polar Oceans". In: Philosophical transactions. Series A, Mathematical, physical, and engineering sciences 372.2025. ISSN: 1364-503X. DOI: 10.1098/rsta.2013.0337.

Adjou, Mohamed, Jørgen Bendtsen, and Katherine Richardson (2012). "Modeling the Influence from Ocean Transport, Mixing and Grazing on Phytoplankton Diversity". In: Ecological Modelling 225, pp. 19-27. ISSN: 0304-3800. DOI: 10.1016/j.ecolmodel.2011.11.005.

Anderson, Laurence A., Dennis J. McGillicuddy, Mathew E. Maltrud, Ivan D. Lima, and Scott C. Doney (2011). "Impact of Eddy-Wind Interaction on Eddy Demographics and Phytoplankton Community Structure in a Model of the North Atlantic Ocean". In: Dynamics of Atmospheres and Oceans. Special issue of Dynamics of Atmospheres and Oceans in honor of Prof. A.R.Robinson 52.1, pp. 80-94. ISSN: 0377-0265. DOI: 10 .1016/j . dynatmoce . 2011.01 .003$.

Ardyna, Mathieu et al. (2017). "Delineating Environmental Control of Phytoplankton Biomass and Phenology in the Southern Ocean". en. In: Geophysical Research Letters 44.10. ISSN: 1944-8007. DOI: 10.1002/2016GL072428.

Armstrong, Robert A., Cindy Lee, John I. Hedges, Susumu Honjo, and Stuart G. Wakeham (2001). "A New, Mechanistic Model for Organic Carbon Fluxes in the Ocean Based on the Quantitative Association of POC with Ballast Minerals". In: Deep Sea Research Part II: Topical Studies in Oceanography. The US JGOFS Synthesis and Modeling Project: Phase 1 49.1, pp. 219-236. ISSN: 0967-0645. DOI: 10.1016/S0967-0645(01)00101-1.

Arrigo, Kevin R., Gert L. van Dijken, and Seth Bushinsky (2008). "Primary Production in the Southern Ocean, 1997-2006". en. In: Journal of Geophysical Research: Oceans 113.C8. ISSN: 2156-2202. DOI: 10.1029/2007JC004551.

Arrigo, Kevin R., Dale H. Robinson, Denise L. Worthen, Brian Schieber, and Michael P. Lizotte (1998). "Bio-Optical Properties of the Southwestern Ross Sea". en. In: Journal of Geophysical Research: Oceans 103.C10, pp. 21683-21695. ISSN: 2156-2202. DOI: 10.1029/ 98JC02157. 
Arrigo, Kevin R. et al. (1999). "Phytoplankton Community Structure and the Drawdown of Nutrients and CO2 in the Southern Ocean". en. In: Science 283.5400, pp. 365-367. ISSN: 0036-8075, 1095-9203. DOI: 10.1126/science. 283.5400.365.

Arrigo, Kevin and Gert van Dijken (2011). "Secular Trends in Arctic Ocean Net Primary Production". In: Journal of Geophysical Research (Oceans) 116. DOI: 10.1029/2011 JC007151.

Asper, Vernon L. and Walker O. Smith (1999). "Particle Fluxes during Austral Spring and Summer in the Southern Ross Sea, Antarctica". en. In: Journal of Geophysical Research: Oceans 104.C3, pp. 5345-5359. ISSN: 2156-2202. DOI: 10.1029/1998JC900067.

Aumont, Oliver and Laurent Bopp (2006). "Globalizing Results from Ocean in Situ Iron Fertilization Studies". en. In: Global Biogeochemical Cycles 20.2. ISSN: 1944-9224. DOI: 10. 1029/2005GB002591.

Ayres, G. P., Jill M. Cainey, R. W. Gillett, and John P. Ivey (1997). "Atmospheric Sulphur and Cloud Condensation Nuclei in Marine Air in the Southern Hemisphere". In: Philosophical Transactions of the Royal Society B: Biological Sciences 352.1350, pp. 203-211. ISSN: 09628436. DOI: $10.1098 /$ rstb.1997.0015.

Azevedo Correia de Souza, João Marcos, Clément De Boyer Montegut, and Pierre-Yves Traon (2011). "Comparison between Three Implementations of Automatic Identification Algorithms for the Qualification and Characterization of Mesoscale Eddies in the South Atlantic Ocean". In: Ocean Science 7, pp. 317-334. DOI: 10.5194/os-7-317-2011.

Barker, Terry and Igor Bashmakov (2007). "Chapter 11: Mitigation from a Cross-Sectoral Perspective - AR4 WGIII". In: Climate Change 200\%: Mitigation. Contribution of Working Group III to the Fourth Assessment Report of the Intergovernmental Panel on Climate Change. Cambridge, United Kingdom and New York, NY, USA.: Cambridge University Press.

Behrenfeld, Michael J. (2010). “Abandoning Sverdrup's Critical Depth Hypothesis on Phytoplankton Blooms". en. In: Ecology 91.4, pp. 977-989. ISSN: 1939-9170. DOI: 10.1890/091207.1.

Behrenfeld, Michael J., Emmanuel Boss, David A. Siegel, and Donald M. Shea (2005). "CarbonBased Ocean Productivity and Phytoplankton Physiology from Space". In: Global Biogeochemical Cycles 19, GB1006. ISSN: 0886-6236. DOI: 10.1029/2004GB002299.

Behrenfeld, Michael J., Scott C. Doney, Ivan D. Lima, Emmanuel S. Boss, and David A. Siegel (2013). "Annual Cycles of Ecological Disturbance and Recovery Underlying the Subarctic Atlantic Spring Plankton Bloom". en. In: DOI: 10.1002/gbc. 20050.

Berner, Robert A. (1990). "Atmospheric Carbon Dioxide Levels over Phanerozoic Time." eng. In: Science (New York, N.Y.) 249.4975, pp. 1382-1386. ISSN: 0036-8075. DOI: $10.1126 /$ science.249.4975.1382. 
- (1991). "A Model for Atmospheric CO 2 over Phanerozoic Time". en. In: American Journal of Science 291.4, pp. 339-376. ISSN: 0002-9599, 1945-452X. DOI: 10.2475/ajs. 291.4 .339.

Beron-Vera, F. J., M. J. Olascoaga, and G. J. Goni (2008). "Oceanic Mesoscale Eddies as Revealed by Lagrangian Coherent Structures". en. In: Geophysical Research Letters 35.12. ISSN: 1944-8007. DOI: 10.1029/2008GL033957.

Beron-Vera, Francisco J., Yan Wang, María J. Olascoaga, Gustavo J. Goni, and George Haller (2013). "Objective Detection of Oceanic Eddies and the Agulhas Leakage". In: Journal of Physical Oceanography 43.7, pp. 1426-1438. ISSN: 0022-3670. DOI: 10.1175/JPO-D-120171.1.

Bertram, Christine (2011). "The Potential of Ocean Iron Fertilization as an Option for Mitigating Climate Change". English. In: Emissions Trading. Institutional Design, Decision Making and Corporate Strategies, pp. 195-207. ISBN: 978-3-642-20591-0. DOI: 10.1007/978-3642-20592-7_12.

Blain, Stéphane et al. (2007). "Effect of Natural Iron Fertilization on Carbon Sequestration in the Southern Ocean". eng. In: Nature 446.7139, pp. 1070-1074. ISSN: 1476-4687. DOI: 10.1038/nature05700.

Böning, C. W., Astrid Dispert, Martin Visbeck, Stephen Rintoul, and Franziska Schwarzkopf (2008). "The Response of the Antarctic Circumpolar Current to Recent Climate Change". In: Nature Geoscience 1. DOI: 10.1038/ngeo362.

Boss, Emanuel S. and Michael J. Behrenfeld (2010). "In Situ Evaluation of the Initiation of the North Atlantic Phytoplankton Bloom". en. In: Geophysical Research Letters 37.18, p. L18603. ISSN: 1944-8007. DOI: 10.1029/2010GL044174.

Boss, Emmanuel S. et al. (2008). "Observations of Pigment and Particle Distributions in the Western North Atlantic from an Autonomous Float and Ocean Color Satellite". en. In: Limnology and Oceanography 53.5part2, pp. 2112-2122. ISSN: 1939-5590. DOI: 10 .4319/ 10.2008.53.5_part_2.2112.

Bowie, A. R. et al. (2016). "Position Analysis: Ocean Fertilisation". en. In: $A C E C R C$, pp. 132. ISSN: 1835-7911.

Bowie, Andrew R et al. (2001). "The Fate of Added Iron during a Mesoscale Fertilisation Experiment in the Southern Ocean". In: Deep Sea Research Part II: Topical Studies in Oceanography. The Southern Ocean Iron Release Experiment (SOIREE) 48.11, pp. 27032743. ISSN: 0967-0645. DOI: 10.1016/S0967-0645(01) 00015-7.

Boyd, Phil (2008). "Implications of Large-Scale Iron Fertilization of the Oceans. Introduction and Synthesis". en. In: Marine Ecology Progress Series 364, pp. 213-218. ISSN: 0171-8630, 1616-1599. DOI: $10.3354 / \mathrm{meps} 07541$. 
Boyd, Philip W. (2002). "Environmental Factors Controlling Phytoplankton Processes in the Southern Ocean1". en. In: Journal of Phycology 38.5, pp. 844-861. ISSN: 1529-8817. DOI: 10.1046/j.1529-8817.2002.t01-1-01203.x.

Boyd, Philip W., Kevin R. Arrigo, R. Strzepek, and G. L. van Dijken (2012). "Mapping Phytoplankton Iron Utilization: Insights into Southern Ocean Supply Mechanisms". In: Journal of Geophysical Research (Oceans) 117, p. C06009. DOI: 10.1029/2011JC007726.

Boyd, Philip W and Scott C Doney (2003). "The Impact of Climate Change and Feedback Processes on the Ocean Carbon Cycle". en. In: Ocean Biogeochemistry: The Role of the Ocean Carbon Cycle in Global Change. Ed. by Michael J. R. Fasham. Global Change - The IGBP Series. Berlin Heidelberg: Springer-Verlag. ISBN: 978-3-540-42398-0.

Boyd, Philip W. and Michael J. Ellwood (2010). "The Biogeochemical Cycle of Iron in the Ocean". En. In: Nature Geoscience 3.10, p. 675. ISSN: 1752-0908. DOI: 10.1038/ngeo964.

Boyd, Philip W. et al. (2001). "Control of Phytoplankton Growth by Iron Supply and Irradiance in the Subantarctic Southern Ocean: Experimental Results from the SAZ Project". en. In: Journal of Geophysical Research: Oceans 106.C12, pp. 31573-31583. ISSN: 2156-2202. DOI: $10.1029 / 2000 \mathrm{JC} 000348$.

Boyd, Philip W. et al. (2007). "Mesoscale Iron Enrichment Experiments 1993-2005: Synthesis and Future Directions". en. In: Science 315.5812, pp. 612-617. ISSN: 0036-8075, 1095-9203. DOI: $10.1126 /$ science.1131669.

Brannigan, L. (2016). "Intense Submesoscale Upwelling in Anticyclonic Eddies". en. In: Geophysical Research Letters 43.7, pp. 3360-3369. ISSN: 1944-8007. DOI: 10. 1002/2016GL067926.

Brzezinski, Mark A., David M. Nelson, Valerie M. Franck, and Daniel E. Sigmon (2001). "Silicon Dynamics within an Intense Open-Ocean Diatom Bloom in the Pacific Sector of the Southern Ocean". In: Deep Sea Research Part II: Topical Studies in Oceanography. US Southern Ocean JGOFS Program (AESOPS) - Part II 48.19, pp. 3997-4018. ISSN: 0967-0645. DOI: 10.1016/S0967-0645(01)00078-9.

Buesseler, Ken O. (1998). "The Decoupling of Production and Particulate Export in the Surface Ocean". en. In: Global Biogeochemical Cycles 12.2, pp. 297-310. ISSN: 1944-9224. DOI: 10. 1029/97GB03366.

Buesseler, Ken O., Scott C. Doney, and Hauke Kite-Powell (2008a). "Exploring Ocean Iron Fertilization: The Scientific, Economic, Legal and Political Basis : Ocean Iron Fertilization". In: Woods Hole, MA. USA: Woods Hole Oceanographic Institution.

Buesseler, Ken O. et al. (2007). "An Assessment of the Use of Sediment Traps for Estimating Upper Ocean Particle Fluxes". en. In: Journal of Marine Research 65, pp. 345-416.

Buesseler, Ken O. et al. (2008b). "Ocean Iron Fertilization-Moving Forward in a Sea of Uncertainty”. en. In: Science 319.5860, pp. 162-162. ISSN: 0036-8075, 1095-9203. DOI: 10.1126/ science.1154305. 
Buongiorno Nardelli, B., S. Guinehut, N. Verbrugge, Y. Cotroneo, E. Zambianchi, and D. Iudicone (2017). "Southern Ocean Mixed-Layer Seasonal and Interannual Variations From Combined Satellite and In Situ Data: SOUTHERN OCEAN MIXED-LAYER VARIABILITY". en. In: Journal of Geophysical Research: Oceans 122.12, pp. 10042-10060. ISSN: 21699275. DOI: 10.1002/2017JC013314.

Carbon Market Monitor (2016). Carbon Market Monitor - America to the Rescue. Review of Global Markets in 2015 and Outlook for 2016-2018. en. Tech. rep. Thomas Reuters.

Carranza, Magdalena M. and Sarah T. Gille (2015). "Southern Ocean Wind-Driven Entrainment Enhances Satellite Chlorophyll-a through the Summer". en. In: Journal of Geophysical Research: Oceans 120.1, pp. 304-323. ISSN: 2169-9291. DOI: 10.1002/2014JC010203.

Cassar, Nicolas et al. (2015). "The Relation of Mixed-Layer Net Community Production to Phytoplankton Community Composition in the Southern Ocean". en. In: Global Biogeochemical Cycles 29.4, pp. 446-462. ISSN: 1944-9224. DOI: 10.1002/2014GB004936.

Chaigneau, Alexis, Gérard Eldin, and Boris Dewitte (2009). "Eddy Activity in the Four Major Upwelling Systems from Satellite Altimetry (1992-2007)". In: Progress in Oceanography. Eastern Boundary Upwelling Ecosystems: Integrative and Comparative Approaches 83.1, pp. 117-123. ISSN: 0079-6611. DOI: 10.1016/j.pocean.2009.07.012.

Chan, Francis et al. (2008). "Emergence of Anoxia in the California Current Large Marine Ecosystem". en. In: Science 319.5865, pp. 920-920. ISSN: 0036-8075, 1095-9203. DOI: 10 . 1126/science.1149016.

Charlson, Robert J., James E. Lovelock, Meinrat O. Andreae, and Stephen G. Warren (1987). "Oceanic Phytoplankton, Atmospheric Sulphur, Cloud Albedo and Climate". en. In: Nature 326.6114, pp. 655-661. ISSN: 1476-4687. DOI: 10.1038/326655a0.

Chassot, Emmanuel et al. (2010). "Global Marine Primary Production Constrains Fisheries Catches". en. In: Ecology Letters 13.4, pp. 495-505. ISSN: 1461-0248. DOI: 10.1111/j .14610248.2010.01443.x.

Chelton, Dudley B., Peter Gaube, Michael G. Schlax, Jeffrey J. Early, and Roger M. Samelson (2011a). "The Influence of Nonlinear Mesoscale Eddies on Near-Surface Oceanic Chlorophyll". eng. In: Science (New York, N.Y.) 334.6054, pp. 328-332. ISSN: 1095-9203. DOI: 10.1126/science. 1208897.

Chelton, Dudley B. and Michael G. Schlax (2016). Mesoscale Eddies in Altimeter Observations of SSH. Tech. rep. Oregon State University.

Chelton, Dudley B., Michael G. Schlax, Michael H. Freilich, and Ralph F. Milliff (2004). "Satellite Measurements Reveal Persistent Small-Scale Features in Ocean Winds". en. In: Science. ISSN: 0036-8075, 1095-9203. DOI: 10.1126/science.1091901. 
Chelton, Dudley B., Michael G. Schlax, and Roger M. Samelson (2011b). "Global Observations of Nonlinear Mesoscale Eddies". In: Progress in Oceanography 91.2, pp. 167-216. ISSN: 00796611. DOI: $10.1016 / \mathrm{j}$. pocean. 2011.01 .002 .

Chisholm, S. and F. Morel (1991). "What Controls Phytoplankton Production in Nutrient-Rich Areas of the Open Sea - American-Society-of-Limnology-and-Oceanography Symposium 22-24 February 1991 San-Marcos, California - Preface". In: Limnology and Oceanography 36, U1507-U1511.

Chisholm, Sallie W. (2000). "Oceanography: Stirring Times in the Southern Ocean". En. In: Nature 407.6805, p. 685. ISSN: 1476-4687. DOI: 10.1038/35037696.

Chisholm, Sallie W., Paul G. Falkowski, and John J. Cullen (2001). "Dis-Crediting Ocean Fertilization". en. In: Science 294.5541, pp. 309-310. ISSN: 0036-8075, 1095-9203. DOI: 10. 1126/science. 1065349.

Clark, Duncan (2012). "Has the Kyoto Protocol Made Any Difference to Carbon Emissions?" en-GB. In: The Guardian. ISSN: 0261-3077.

IPPC AR5 (2014). Climate Change 2014 Synthesis Report: Summary for Policy Makers. Tech. rep. Intergovernmental Panel on Climate Change.

Cohen, Yuval and Louis I. Gordon (1979). "Nitrous Oxide Production in the Ocean". en. In: Journal of Geophysical Research: Oceans 84.C1, pp. 347-353. ISSN: 2156-2202. DOI: 10.1029/JC084iC01p00347.

Comiso, Josefino C. (2011). "Large Decadal Decline of the Arctic Multiyear Ice Cover". In: Journal of Climate 25.4, pp. 1176-1193. ISSN: 0894-8755. DOI: 10 . 1175/ JCLI - D - 11 00113.1.

Comiso, Josefino C., Donald J. Cavalieri, Claire L. Parkinson, and Per Gloersen (1997). "Passive Microwave Algorithms for Sea Ice Concentration: A Comparison of Two Techniques". In: Remote Sensing of Environment 60.3, pp. 357-384. ISSN: 0034-4257. DOI: 10.1016/ S0034-4257 (96)00220-9.

Cullen, John J. and Philip W. Boyd (2008). "Predicting and Verifying the Intended and Unintended Consequences of Large-Scale Ocean Iron Fertilization". en. In: Marine Ecology Progress Series 364, pp. 295-301. ISSN: 0171-8630, 1616-1599. DOI: 10.3354/meps07551.

Dale, Trine, Francisco Rey, and Berit Riddervold Heimdal (1999). "Seasonal Development of Phytoplankton at a High Latitude Oceanic Site". In: Sarsia 84.5-6, pp. 419-435. ISSN: 0036-4827. DOI: $10.1080 / 00364827.1999 .10807347$.

Dawson, Hannah R. S., Peter G. Strutton, and Peter Gaube (2018). "The Unusual Surface Chlorophyll Signatures of Southern Ocean Eddies". en. In: Journal of Geophysical Research: Oceans 123.9, pp. 6053-6069. ISSN: 2169-9291. DOI: 10.1029/2017JC013628.

de Baar, Hein J. W. et al. (1995). "Importance of Iron for Plankton Blooms and Carbon Dioxide Drawdown in the Southern Ocean". In: Nature 373, pp. 412-415. 
de Baar, Hein J. W. et al. (2005). "Synthesis of Iron Fertilization Experiments: From the Iron Age in the Age of Enlightenment". en. In: Journal of Geophysical Research: Oceans 110.C9, C09S16. ISSN: 2156-2202. DOI: 10.1029/2004JC002601.

de la Rocha, Christina L. (2006). "Chapter 5. The Biological Pump". In: The Oceans and Marine Geochemistry - 1st Edition. 1st Edition. Pergamon. ISBN: 978-0-08-091438-1.

Denman, Kenneth L. (2008). "Climate Change, Ocean Processes and Ocean Iron Fertilization". en. In: Marine Ecology Progress Series 364, pp. 219-225. ISSN: 0171-8630, 1616-1599. DOI: 10.3354/meps07542.

Deppeler, Stacy L. and Andrew T. Davidson (2017). "Southern Ocean Phytoplankton in a Changing Climate". English. In: Frontiers in Marine Science 4. ISSN: 2296-7745. DOI: 10. 3389/fmars. 2017.00040.

Dewar, William K. and Glenn R. Flierl (1987). "Some Effects of the Wind on Rings". In: Journal of Physical Oceanography 17.10, pp. 1653-1667. ISSN: 0022-3670. DOI: $10.1175 / 1520$ 0485(1987) 017<1653: SEOTWO>2 . 0.CO; 2.

Dierssen, Heidi M. and Ray C. Smith (2000). "Bio-Optical Properties and Remote Sensing Ocean Color Algorithms for Antarctic Peninsula Waters". en. In: Journal of Geophysical Research: Oceans 105.C11, pp. 26301-26312. ISSN: 2156-2202. DOI: 10.1029/1999JC000296.

DiTullio, G. R. et al. (2000). "Rapid and Early Export of Phaeocystis Antarctica Blooms in the Ross Sea, Antarctica". En. In: Nature 404.6778, p. 595. ISSN: 1476-4687. DOI: 10.1038/ 35007061 .

Doddridge, Edward W. and David P. Marshall (2018). "Implications of Eddy Cancellation for Nutrient Distribution Within Subtropical Gyres". en. In: Journal of Geophysical Research: Oceans 123.9, pp. 6720-6735. ISSN: 2169-9291. DOI: 10.1029/2018JC013842.

Doney, Scott C., Victoria J. Fabry, Richard A. Feely, and Joan A. Kleypas (2009a). "Ocean Acidification: The Other CO2 Problem". In: Annual Review of Marine Science 1.1, pp. 169192. ISSN: 1941-1405. DOI: 10.1146/annurev.marine.010908.163834.

Doney, Scott C., David M. Glover, Scott J. McCue, and Montserrat Fuentes (2003). "Mesoscale Variability of Sea-Viewing Wide Field-of-View Sensor (SeaWiFS) Satellite Ocean Color: Global Patterns and Spatial Scales". en. In: Journal of Geophysical Research: Oceans 108.C2, p. 3024. ISSN: 2156-2202. DOI: 10.1029/2001JC000843.

Doney, Scott C., David M. Glover, and Raymond G. Najjar (1996). "A New Coupled, OneDimensional Biological-Physical Model for the Upper Ocean: Applications to the JGOFS Bermuda Atlantic Time-Series Study (BATS) Site". In: Deep Sea Research Part II: Topical Studies in Oceanography 43.2, pp. 591-624. ISSN: 0967-0645. DOI: 10.1016/0967-0645(95) 00104-2.

Doney, Scott C. et al. (2009b). "Skill Metrics for Confronting Global Upper Ocean EcosystemBiogeochemistry Models against Field and Remote Sensing Data". In: Journal of Marine 199 
Systems. Skill assessment for coupled biological/physical models of marine systems 76.1 , pp. 95-112. ISSN: 0924-7963. DOI: 10.1016/j . jmarsys.2008.05.015.

Dong, Changming, Francesco Nencioli, Yu Liu, and James C. McWilliams (2011). "An Automated Approach to Detect Oceanic Eddies From Satellite Remotely Sensed Sea Surface Temperature Data". In: Geoscience and Remote Sensing Letters, IEEE 8, pp. 1055-1059. DOI: $10.1109 /$ LGRS . 2011.2155029.

Dong, Shenfu, Janet Sprintall, Sarah T. Gille, and Lynne Talley (2008). "Southern Ocean Mixed-Layer Depth from Argo Float Profiles". en. In: Journal of Geophysical Research: Oceans 113.C6. ISSN: 2156-2202. DOI: 10.1029/2006JC004051.

d'Ovidio, Francesco et al. (2015). "The Biogeochemical Structuring Role of Horizontal Stirring: Lagrangian Perspectives on Iron Delivery Downstream of the Kerguelen Plateau". English. In: Biogeosciences 12.19, pp. 5567-5581. ISSN: 1726-4170. DOI: https ://doi .org/10 . $5194 / \mathrm{bg}-12-5567-2015$.

Downes, Stephanie M. et al. (2015). "An Assessment of Southern Ocean Water Masses and Sea Ice during 1988-2007 in a Suite of Interannual CORE-II Simulations". In: Ocean Modelling 94, pp. 67-94. ISSN: 1463-5003. DOI: 10.1016/j.ocemod.2015.07.022.

Duarte, Pedro et al. (2017). "Sea Ice Thermohaline Dynamics and Biogeochemistry in the Arctic Ocean: Empirical and Model Results". en. In: Journal of Geophysical Research: Biogeosciences 122.7, pp. 1632-1654. ISSN: 2169-8961. DOI: 10.1002/2016JG003660.

Dubischar, Corinna D. and Ulrich V. Bathmann (1997). "Grazing Impact of Copepods and Salps on Phytoplankton in the Atlantic Sector of the Southern Ocean". In: Deep Sea Research Part II: Topical Studies in Oceanography 44.1, pp. 415-433. ISSN: 0967-0645. DOI: 10.1016/S0967-0645(96)00064-1.

Dufois, François, Nick J. Hardman-Mountford, Jim Greenwood, Anthony J. Richardson, Ming Feng, and Richard J. Matear (2016). "Anticyclonic Eddies Are More Productive than Cyclonic Eddies in Subtropical Gyres Because of Winter Mixing". en. In: Science Advances 2.5, e1600282. ISSN: 2375-2548. DOI: 10.1126/sciadv.1600282.

Dufois, François et al. (2014). "Impact of Eddies on Surface Chlorophyll in the South Indian Ocean". en. In: Journal of Geophysical Research: Oceans 119.11, pp. 8061-8077. ISSN: 21699291. DOI: $10.1002 / 2014$ JC010164.

Early, Jeffrey J., R. M. Samelson, and Dudley B. Chelton (2011). "The Evolution and Propagation of Quasigeostrophic Ocean Eddies". In: Journal of Physical Oceanography 41.8, pp. 1535-1555. ISSN: 0022-3670. DOI: 10.1175/2011JP04601.1.

Ellertsen, Hans Chr (1993). "Spring Blooms and Stratification". En. In: Nature 363.6424, p. 24. ISSN: 1476-4687. DOI: 10.1038/363024a0.

Elzinga, Aant (1993). "Anarctica: The Construction of a Continent by and for Science". en. In: Denationalizing Science: The Contexts of International Scientific Practice. Ed. by E. 
Crawford, T. Shinn, and Sverker Sörlin. Sociology of the Sciences Yearbook. Springer Netherlands. ISBN: 978-0-7923-1855-2.

Faghmous, James H., Ivy Frenger, Yuanshun Yao, Robert Warmka, Aron Lindell, and Vipin Kumar (2015). "A daily global mesoscale ocean eddy dataset from satellite altimetry". English (US). In: Scientific data 2. DOI: 10.1038/sdata.2015.28.

Falkowski, Paul G. (1997). "Evolution of the Nitrogen Cycle and Its Influence on the Biological Sequestration of CO2 in the Ocean". en. In: Nature 387.6630, pp. 272-275. ISSN: 1476-4687. DOI: $10.1038 / 387272 \mathrm{a} 0$.

- (2002). "The Ocean's Invisible Forest". eng. In: Scientific American 287.2, pp. 54-61. ISSN: 0036-8733.

Falkowski, Paul G., David Ziemann, Zbigniew Kolber, and Paul K. Bienfang (1991). "Role of Eddy Pumping in Enhancing Primary Production in the Ocean". En. In: Nature 352.6330, p. 55. ISSN: 1476-4687. DOI: 10.1038/352055a0.

Falkowski, Paul et al. (2000). "The Global Carbon Cycle: A Test of Our Knowledge of Earth as a System". en. In: Science 290.5490, pp. 291-296. ISSN: 0036-8075, 1095-9203. DOI: 10.1126/science.290.5490.291.

Fasham, Michael J. R., Hugh W. Ducklow, and Stuart M. McKelvie (1990). "A Nitrogen-Based Model of Plankton Dynamics in the Oceanic Mixed Layer". In: Journal of Marine Research 48.3, pp. 591-639. DOI: 10.1357/002224090784984678.

Fauchereau, Nicolas, Alessandro Tagliabue, Laurent Bopp, and Pedro M. S. Monteiro (2011). "The Response of Phytoplankton Biomass to Transient Mixing Events in the Southern Ocean". In: Geophysical Research Letters 38, p. L17601. ISSN: 0094-8276. DOI: 10 . 1029/ 2011 GL048498.

Feely, Richard A., Scott C. Doney, and Sarah R. Cooley (2009). "Ocean Acidification: Present Conditions and Future Changes in a High-CO2 World". In: Oceanography. Issn: 1042-8275.

Feldmann, Johannes and Anders Levermann (2015). "Collapse of the West Antarctic Ice Sheet after Local Destabilization of the Amundsen Basin". en. In: Proceedings of the National Academy of Sciences 112.46, pp. 14191-14196. ISSN: 0027-8424, 1091-6490. DOI: 10.1073/ pnas. 1512482112.

Fennel, Katja, Mark Abbott, Yh Spitz, James Richman, and David M. Nelson (2003). "Impacts of Iron Control on Phytoplankton Production in the Modern and Glacial Southern Ocean". In: Deep-Sea Research II 50, pp. 833-851. DOI: 10.1016/S0967-0645(02)00596-9.

Ferrari, Raffaele and Carl Wunsch (2008). "Ocean Circulation Kinetic Energy: Reservoirs, Sources, and Sinks". In: Annual Review of Fluid Mechanics 41.1, pp. 253-282. ISSN: 00664189. DOI: 10.1146/annurev.fluid.40.111406.102139. 
Fiksen, Ø (2000). "The Adaptive Timing of Diapause - a Search for Evolutionarily Robust Strategies in Calanus Finmarchicus". In: ICES Journal of Marine Science 57.6, pp. 18251833. ISSN: 1054-3139. DOI: 10.1006/jmsc.2000.0976.

Fischer, Alexis et al. (2014). "Sixty Years of Sverdrup: A Retrospective of Progress in the Study of Phytoplankton Blooms". en. In: Oceanography Society. ISSN: 10428275.

Flierl, Glenn R. (1981). "Particle Motions in Large-Amplitude Wave Fields". In: Geophysical \& Astrophysical Fluid Dynamics 18.1-2, pp. 39-74. ISSN: 0309-1929. DOI: 10.1080 / 03091928108208773.

Frankignoulle, Michel, Christine Canon, and Jean-Pierre Gattuso (1994). "Marine Calcification as a Source of Carbon Dioxide: Positive Feedback of Increasing Atmospheric CO2". en. In: Limnology and Oceanography 39.2, pp. 458-462. ISSN: 1939-5590. DOI: 10.4319/1o.1994. 39.2 .0458$.

Franks, Peter J. S., Joe S. Wroblewski, and Glenn R. Flierl (1986). "Prediction of Phytoplankton Growth in Response to the Frictional Decay of a Warm-Core Ring". en. In: Journal of Geophysical Research: Oceans 91.C6, pp. 7603-7610. ISSN: 2156-2202. DOI: 10 .1029/ JC091iC06p07603.

Frenger, Ivy, Matthias Münnich, and Nicolas Gruber (2018). "Imprint of Southern Ocean Eddies on Chlorophyll". In: Biogeosciences Discussions, pp. 1-26. DOI: 10.5194/bg-201870.

Frenger, Ivy, Matthias Münnich, Nicolas Gruber, and Reto Knutti (2015). "Southern Ocean Eddy Phenomenology". en. In: Journal of Geophysical Research: Oceans 120.11, pp. 74137449. ISSN: 2169-9291. DOI: 10.1002/2015JC011047.

Fu, Lee-Lueng, Dudley Chelton, Pierre-Yves Le Traon, and Rosemary Morrow (2010). "Eddy Dynamics From Satellite Altimetry". In: Oceanography 23.4, pp. 14-25. ISSN: 10428275. DOI: $10.5670 /$ oceanog. 2010.02.

Full Committee Hearing - Geoengineering, Part I (2009). Full Committee Hearing - Geoengineering, Part I: Assessing the Implications of Large-Scale Climate Intervention. en. Washington, D. C.

Gaiero, Diego M., Jean-Luc Probst, Pedro J. Depetris, Susana M. Bidart, and Lydia Leleyter (2003). "Iron and Other Transition Metals in Patagonian Riverborne and Windborne Materials: Geochemical Control and Transport to the Southern South Atlantic Ocean". en. In: Geochimica et Cosmochimica Acta vol. 6.n 19, pp. 3603-3623. ISSN: 0016-7037.

Garver, Sara A. and David A. Siegel (1997). "Inherent Optical Property Inversion of Ocean Color Spectra and Its Biogeochemical Interpretation: 1. Time Series from the Sargasso Sea". en. In: Journal of Geophysical Research: Oceans 102.C8, pp. 18607-18625. ISSN: 2156-2202. DOI: $10.1029 / 96 \mathrm{JC} 03243$. 
Gaube, Peter, Dudley B. Chelton, Roger M. Samelson, Michael G. Schlax, and Larry W. O’Neill (2014a). "Satellite Observations of Mesoscale Eddy-Induced Ekman Pumping". In: Journal of Physical Oceanography 45.1, pp. 104-132. ISSN: 0022-3670. DOI: 10.1175/JPOD-14-0032.1.

Gaube, Peter, Dudley B. Chelton, Peter G. Strutton, and Michael J. Behrenfeld (2013). "Satellite Observations of Chlorophyll, Phytoplankton Biomass, and Ekman Pumping in Nonlinear Mesoscale Eddies". en. In: Journal of Geophysical Research: Oceans 118.12, pp. 63496370. ISSN: 2169-9291. DOI: 10.1002/2013JC009027.

Gaube, Peter and Dennis J. McGillicuddy (2017). "The Influence of Gulf Stream Eddies and Meanders on Near-Surface Chlorophyll". In: Deep Sea Research Part I: Oceanographic Research Papers 122, pp. 1-16. ISSN: 0967-0637. DOI: 10.1016/j.dsr.2017.02.006.

Gaube, Peter, Dennis Mcgillicuddy, Dudley B. Chelton, Michael J. Behrenfeld, and Peter G. Strutton (2014b). "Regional Variations in the Influence of Mesoscale Eddies on NearSurface Chlorophyll". In: Journal of Geophysical Research: Oceans 119. DOI: 10.1002/ $2014 \mathrm{JC} 010111$.

Geider, Richard, Hugh Macintyre, and Todd Kana (1998). "A Dynamic Regulatory Model of Phytoplanktonic Acclimation to Light, Nutrients, and Temperature". In: Limnology and Oceanography - LIMNOL OCEANOGR 43, pp. 679-694. DOI: 10.4319/1o.1998.43.4. 0679.

Gille, Sarah T. (2002). "Warming of the Southern Ocean Since the 1950s". en. In: Science 295.5558, pp. 1275-1277. ISSN: 0036-8075, 1095-9203. DOI: 10.1126/science.1065863.

Gillenwater, Michael (2012). What Is Additionality? Part 2: A Framework for More Precise Definitions and Standardized Approaches. en. Tech. rep. Silver Spring, MD, USA: Greenhouse Gas Management Institute, p. 31.

Gillenwater, Michael and Stephen Seres (2011). The Clean Development Mechanism: A Review of the First International Offset Program. Tech. rep. Center For Climate and Energy Solutions.

Glover, David M., Scott C. Doney, William K. Oestreich, and Alisdair W. Tullo (2018). "Geostatistical Analysis of Mesoscale Spatial Variability and Error in SeaWiFS and MODIS/ Aqua Global Ocean Color Data". en. In: Journal of Geophysical Research: Oceans 123.1, pp. 22-39. ISSN: 2169-9291. DOI: 10.1002/2017JC013023.

Gnanadesikan, Anand and Irina Marinov (2008). "Export Is Not Enough : Nutrient Cycling and Carbon Sequestration". en. In: DOI: 10.3354/meps07550.

Gnanadesikan, Anand, Jorge L. Sarmiento, and Richard D. Slater (2003). "Effects of Patchy Ocean Fertilization on Atmospheric Carbon Dioxide and Biological Production". In: Global Biogeochemical Cycles 17, p. 1050. ISSN: 0886-6236. DOI: 10.1029/2002GB001940. 
Goodell, Jeff (2011a). "A Little Cash on the Side". In: How to Cool the Planet Geoengineering and the Audacious Quest to Fix Earth's Climate. First Mariner Books edition. Boston: Mariner Books, p. 160.

- (2011b). "A Little Cash on the Side". In: How to Cool the Planet Geoengineering and the Audacious Quest to Fix Earth's Climate. First Mariner Books edition. Boston: Mariner Books, p. 145.

- (2011c). "A Little Cash on the Side". In: How to Cool the Planet Geoengineering and the Audacious Quest to Fix Earth's Climate. First Mariner Books edition. Boston: Mariner Books, pp. 150-160.

- (2011d). "A Little Cash on the Side". In: How to Cool the Planet Geoengineering and the Audacious Quest to Fix Earth's Climate. First Mariner Books edition. Boston: Mariner Books, p. 161.

Gould, John et al. (2004). "Argo Profiling Floats Bring New Era of in Situ Ocean Observations". en. In: Eos, Transactions American Geophysical Union 85.19, pp. 185-191. ISSN: 2324-9250. DOI: $10.1029 / 2004 E 0190002$.

Gran, Haaken H. and Trygve Braarud (1935). "A Quantitative Study of the Phytoplankton in the Bay of Fundy and the Gulf of Maine (Including Observations on Hydrography, Chemistry and Turbidity)". In: Journal of the Biological Board of Canada 1.5, pp. 279467. ISSN: 0368-1424. DOI: 10.1139/f35-012.

Grantham, Brian A. et al. (2004). "Upwelling-Driven Nearshore Hypoxia Signals Ecosystem and Oceanographic Changes in the Northeast Pacific". eng. In: Nature 429.6993, pp. 749 754. ISSN: 1476-4687. DOI: 10.1038/nature02605.

Griffies, Stephen et al. (2009). "Coordinated Ocean-Ice Reference Experiments (COREs)". en. In: Ocean Modelling 26.1-2, pp. 1-46. ISSN: 1463-5003. DOI: 10.1016/j.ocemod.2008.08. 007.

Haller, George (2015). "Lagrangian Coherent Structures". In: Annual Review of Fluid Mechanics 47.1, pp. 137-162. ISSN: 0066-4189. DOI: 10.1146/annurev-fluid-010313-141322.

Hamrick, Kelly and Melissa Gallant (2017). Unlocking Potential. State of the Voluntary Carbon Markets 2017. en-US. Annual Review. Forest Trends' Ecosytem Marketplace.

Hamrick, Kelly and Allie Goldstein (2016). Raising Ambition. State of the Voluntary Carbon Markets 2016. en. Tech. rep. Forest Trends' Ecosystem Marketplace.

Handbook of the Antarctic Treaty System (n.d.). https://www.state.gov/e/oes/rls/rpts/ant/. Harrison, Cheryl S., Matthew C. Long, Nicole S. Lovenduski, and Jefferson K. Moore (2018). "Mesoscale Effects on Carbon Export: A Global Perspective". In: Global Biogeochemical Cycles 0.0. ISSN: 0886-6236. DOI: 10.1002/2017GB005751. 
Harrison, Daniel P. (2013). "A Method for Estimating the Cost to Sequester Carbon Dioxide by Delivering Iron to the Ocean". In: International Journal of Global Warming 5.3, pp. 231254. ISSN: 1758-2083. DOI: 10.1504/IJGW. 2013. 055360.

Hauck, Judith et al. (2015). "On the Southern Ocean CO2 Uptake and the Role of the Biological Carbon Pump in the 21st Century". en. In: Global Biogeochemical Cycles 29.9, 2015GB005140. ISSN: 1944-9224. DOI: 10.1002/2015GB005140.

Hausmann, Ute, Dennis J. McGillicuddy, and John Marshall (2017). "Observed Mesoscale Eddy Signatures in Southern Ocean Surface Mixed-Layer Depth". en. In: Journal of Geophysical Research: Oceans 122.1, pp. 617-635. ISSN: 2169-9291. DOI: 10.1002/2016JC012225.

Hoffmann, Linn J., Ilka Peeken, Karin Lochte, Philipp Assmy, and Marcel Veldhuis (2006). "Different Reactions of Southern Ocean Phytoplankton Size Classes to Iron Fertilization". en. In: Limnology and Oceanography 51.3, pp. 1217-1229. ISSN: 1939-5590. DOI: 10.4319/ 10.2006 .51 .3 .1217$.

Holling, Crawford S. (1959). "Some Characteristics of Simple Types of Predation and Parasitism". In: The Canadian Entomologist 91.7, pp. 385-398. ISSN: 1918-3240, 0008-347X. DOI: $10.4039 /$ Ent91385-7.

Hovi, Jon, Detlef F. Sprinz, and Arild Underdal (2009). "Implementing Long-Term Climate Policy: Time Inconsistency, Domestic Politics, International Anarchy". en. In: Global Environmental Politics 9.3, pp. 20-39. ISSN: 1536-0091.

Howkins, Adrian (2010). "Science, Environment, and Sovereignty: The International Geophysical Year in the Antarctic Peninsula Region". en. In: Globalizing Polar Science: Reconsidering the International Polar and Geophysical Years. Ed. by Roger D. Launius, James Rodger Fleming, and David H. DeVorkin. Palgrave Studies in the History of Science and Technology. New York: Palgrave Macmillan US, pp. 245-264. ISBN: 978-0-230-11465-4. DOI: 10.1057/9780230114654_14.

Huisman, Jef, Paul van Oostveen, and Franz J. Weissing (1999). "Critical Depth and Critical Turbulence: Two Different Mechanisms for the Development of Phytoplankton Blooms". en. In: Limnology and Oceanography 44.7, pp. 1781-1787. ISSN: 1939-5590. DOI: 10.4319/ 1o.1999.44.7.1781.

IPCC AR4 (2007). Human and Natural Drivers of Climate Change - AR4 WGI Summary for Policymakers. Tech. rep. IPCC.

Hunke, Elizabeth C. and William H. Lipscomb (2008). CICE: The Los Alamos Sea Ice Model. Documentation and Software User's Manual. Version 4.0.

Hurrell, James W. et al. (2013). "The Community Earth System Model: A Framework for Collaborative Research". In: Bulletin of the American Meteorological Society 94.9, pp. 13391360. ISSN: 0003-0007. DOI: 10.1175/BAMS-D-12-00121.1. 
Hutchins, David A. and Kenneth W. Bruland (1998). "Iron-Limited Diatom Growth and Si:N Uptake Ratios in a Coastal Upwelling Regime". en. In: Nature 393.6685, pp. 561-564. ISSN: 1476-4687. DOI: $10.1038 / 31203$.

Iida, Takahiro and Tsuneo Odate (2014). "Seasonal Variability of Phytoplankton Biomass and Composition in the Major Water Masses of the Indian Ocean Sector of the Southern Ocean". In: Polar Science 8.3, pp. 283-297. ISSN: 1873-9652. DOI: 10.1016/j ·polar.2014.03.003. IMO (2008). Note by the International Maritime Organization (IMO) Input to the FCCC - The Assembly Document. Annex III Ocean Fertilization and CO2 Sequestration in Sub-Seabed Geological Formations. Tech. rep. International Maritime Organization.

Isern-Fontanet, Jordi, Emilio García-Ladona, Jordi Font, Jordi Isern-Fontanet, Emilio GarcíaLadona, and Jordi Font (2003). Identification of Marine Eddies from Altimetric Maps. EN. https://journals.ametsoc.org/doi/abs/10.1175/1520-0426\%282003\%2920\%3C772\%3AIOMEFA\%3E2.0.CO\% Research-Article.

Jang, Chan Joo, Jisoo Park, Taewook Park, and Sinjae Yoo (2011). "Response of the Ocean Mixed Layer Depth to Global Warming and Its Impact on Primary Production: A Case for the North Pacific Ocean". en. In: ICES Journal of Marine Science 68.6, pp. 996-1007. ISSN: 1054-3139. DOI: 10.1093/icesjms/f sr064.

Jin, Xin and Nicolas Gruber (2003). "Offsetting the Radiative Benefit of Ocean Iron Fertilization by Enhancing N2O Emissions". en. In: Geophysical Research Letters 30.24. ISSN: 1944-8007. DOI: 10.1029/2003GL018458.

Johnson, Kenneth S., R. Michael Gordon, and Kenneth H. Coale (1997). "What Controls Dissolved Iron Concentrations in the World Ocean?" In: Marine Chemistry 57.3, pp. 137161. ISSN: 0304-4203. DOI: 10.1016/S0304-4203(97)00043-1.

Karl, David M. and Bronte D. Tilbrook (1994). "Production and Transport of Methane in Oceanic Particulate Organic Matter". en. In: Nature 368.6473, pp. 732-734. ISSN: 14764687. DOI: $10.1038 / 368732 \mathrm{a} 0$.

Kay, Jennifer E. et al. (2014). "The Community Earth System Model (CESM) Large Ensemble Project: A Community Resource for Studying Climate Change in the Presence of Internal Climate Variability". In: Bulletin of the American Meteorological Society 96.8, pp. 13331349. ISSN: 0003-0007. DOI: 10.1175/BAMS-D-13-00255.1.

Keith, David (2013). A Case for Climate Engineering. English. Cambridge, M ass: The MIT Press. ISBN: 978-0-262-01982-8.

Keith, David W., Minh Ha-Duong, and Joshuah K. Stolaroff (2006). "Climate Strategy with Co2 Capture from the Air". en. In: Climatic Change 74.1-3, pp. 17-45. ISSN: 0165-0009, 1573-1480. DOI: 10.1007/s10584-005-9026-x. 
Lancelot, Christiane et al. (2009). "Spatial Distribution of the Iron Supply to Phytoplankton in the Southern Ocean: A Model Study". In: Biogeosciences 6.12, pp. 2861-2878. ISSN: 1726-4189. DOI: $10.5194 / \mathrm{bg}-6-2861-2009$.

Large, Willaim G., James C. McWilliams, and Scott C. Doney (1994). "Oceanic Vertical Mixing: A Review and a Model with a Nonlocal Boundary Layer Parameterization". en. In: Reviews of Geophysics 32.4, pp. 363-403. ISSN: 1944-9208. DOI: 10.1029/94RG01872.

Large, William G. (1998). "Modeling and Parameterizing the Ocean Planetary Boundary Layer". en. In: Ocean Modeling and Parameterization. NATO Science Series. Springer, Dordrecht, pp. 81-120. ISBN: 978-94-011-5096-5. DOI: 10.1007/978-94-011-5096-5_3.

Large, William G. and Stephen Yeager (2004). "Diurnal to Decadal Global Forcing for Ocean and Sea-Ice Models: The Data Sets and Flux Climatologies". en. In: DOI: 10 . 5065 / D6KK98Q6.

Laufkötter, Charlotte et al. (2015). "Drivers and Uncertainties of Future Global Marine Primary Production in Marine Ecosystem Models". In: Biogeosciences 12.23, pp. 6955-6984. ISSN: 1726-4189. DOI: $10.5194 / \mathrm{bg}-12-6955-2015$.

Law, Cliff S. (2008). "Predicting and Monitoring the Effects of Large-Scale Ocean Iron Fertilization on Marine Trace Gas Emissions". en. In: Marine Ecology Progress Series 364, pp. 283-288. ISSN: 0171-8630, 1616-1599. DOI: 10.3354/meps07549.

Laws, Edward A., Paul G. Falkowski, Walker O. Smith, Hugh Ducklow, and James J. McCarthy (2000). "Temperature Effects on Export Production in the Open Ocean". In: Global Biogeochemical Cycles 14.4, pp. 1231-1246. ISSN: 1944-9224. DOI: 10.1029/1999GB001229.

Lehahn, Yoav, Francesco d'Ovidio, Marina Lévy, Yael Amitai, and Eyal Heifetz (2011). "Long Range Transport of a Quasi Isolated Chlorophyll Patch by an Agulhas Ring". en. In: Geophysical Research Letters 38.16, p. L16610. ISSN: 1944-8007. DOI: 10.1029/2011GL048588.

Leinen, Margaret (2008). "Building Relationships between Scientists and Business in Ocean Iron Fertilization". In: Marine Ecology Progress Series 364, pp. 251-256. ISSN: 0171-8630.

Levasseur, Maurice et al. (2006). "DMSP and DMS Dynamics during a Mesoscale Iron Fertilization Experiment in the Northeast Pacific-Part I: Temporal and Vertical Distributions". In: Deep Sea Research Part II: Topical Studies in Oceanography. Canadian SOLAS: Subarctic Ecosystem Response to Iron Enrichment (SERIES) 53.20, pp. 2353-2369. ISSN: 0967-0645. DOI: $10.1016 / j$.dsr2.2006.05.023.

Lima, Ivan D., Phoebe J. Lam, and Scott C. Doney (2014). "Dynamics of Particulate Organic Carbon Flux in a Global Ocean Model". In: Biogeosciences 11.4, pp. 1177-1198. ISSN: 1726-4189. DOI: 10.5194/bg-11-1177-2014.

Liss, Peter, Adele Chuck, Dorothee Bakker, and Suzanne Turner (2005). "Ocean Fertilization with Iron: Effects on Climate and Air Quality". en. In: Tellus B 57.3, pp. 269-271. ISSN: 1600-0889. DOI: $10.1111 / \mathrm{j} .1600-0889.2005 .00141 . \mathrm{x}$. 
Long, Matthew C., Keith Lindsay, and Marika M. Holland (2015). "Modeling Photosynthesis in Sea Ice-Covered Waters". en. In: Journal of Advances in Modeling Earth Systems 7.3, pp. 1189-1206. ISSN: 1942-2466. DOI: 10.1002/2015MS000436.

Long, Matthew C., Keith Lindsay, Synte Peacock, J. Keith Moore, and Scott C. Doney (2013). "Twentieth-Century Oceanic Carbon Uptake and Storage in CESM1(BGC)". In: Journal of Climate 26.18, pp. 6775-6800. ISSN: 0894-8755. DOI: 10.1175/JCLI-D-12-00184.1.

Longhurst, Alan R. (2006). Ecological Geography of the Sea, Second Edition. English. 2 edition. Oxford ; Boston: Academic Press. IsBn: 978-0-7506-1158-9.

Lukacs, Martin (2012). "World's Biggest Geoengineering Experiment 'violates' UN Rules". en-GB. In: The Guardian. ISSN: 0261-3077.

Lynas, Mark (2011). The God Species: Saving the Planet in the Age of Humans. English. Washington, D.C: National Geographic. ISBN: 978-1-4262-0891-1.

Mahadevan, Amala, Leif N. Thomas, and Amit Tandon (2008). "Comment on "Eddy/Wind Interactions Stimulate Extraordinary Mid-Ocean Plankton Blooms"". eng. In: Science (New York, N.Y.) 320.5875, 448, author reply 448. ISSN: 1095-9203. DOI: $10.1126 /$ science. 1152111.

Mangel, Marc (2016). "Whales, Science, and Scientific Whaling in the International Court of Justice". In: Proceedings of the National Academy of Sciences of the United States of America 113.51, pp. 14523-14527. ISSN: 0027-8424. DOI: 10.1073/pnas.1604988113.

Marchetti, Adrian, Nelson D. Sherry, Hiroshi Kiyosawa, Atsushi Tsuda, and Paul J. Harrison (2006). "Phytoplankton processes during a mesoscale iron enrichment in the NE subarctic Pacific: Part I-Biomass and assemblage". English (US). In: Deep-Sea Research Part II: Topical Studies in Oceanography 53.20-22, pp. 2095-2113. ISSN: 0967-0645. DOI: 10.1016/ j.dsr2.2006.05.038.

Marinov, Irina, Anand Gnanadesikan, Jorge L. Sarmiento, J. R. Toggweiler, Michael J. Follows, and B. K. Mignone (2008). "Impact of Oceanic Circulation on Biological Carbon Storage in the Ocean and Atmospheric pCO2". en. In: DOI: 10.1029/2007GB002958.

Markels, Michael and Richard T. Barber (2002). "Sequestration of Carbon Dioxide by Ocean Fertilization". en. In: Environmental Challenges and Greenhouse Gas Control for Fossil Fuel Utilization in the 21st Century. Springer, Boston, MA, pp. 119-131. ISBN: 978-14613-5232-7. DOI: 10.1007/978-1-4615-0773-4_9.

Martin, Adrian and Kelvin J. Richards (2001). "Mechanisms for Vertical Nutrient Transport within North Atlantic Mesoscale Eddy". In: Deep-sea Research Part Ii-topical Studies in Oceanography - DEEP-SEA RES PT II-TOP ST OCE 48, pp. 757-773. DOI: 10.1016/ S0967-0645(00)00096-5.

Martin, John H. (1990). "Glacial-Interglacial CO2 Change: The Iron Hypothesis". In: Paleoceanography 5, pp. 1-13. ISSN: 0883-8305. DOI: $10.1029 /$ PA005i001p00001. 
Martin, John H. and Steve E. Fitzwater (1988). "Iron Deficiency Limits Phytoplankton Growth in the North-East Pacific Subarctic". En. In: Nature 331.6154, p. 341. ISSN: 1476-4687. DOI: 10.1038/331341a0.

Martin, John H., Steve E. Fitzwater, and R. Michael Gordon (1990). "Iron Deficiency Limits Phytoplankton Growth in Antarctic Waters". en. In: Global Biogeochemical Cycles 4.1, pp. 5-12. ISSN: 1944-9224. DOI: 10.1029/GB004i001p00005.

Martin, John H., Michael Gordon, and Steve E. Fitzwater (1991). "The Case for Iron". en. In: Limnology and Oceanography 36.8, pp. 1793-1802. ISSN: 1939-5590. DOI: 10.4319/1o. 1991.36 .8 .1793$.

Mazloff, Matthew R., Patrick Heimbach, and Carl Wunsch (2010). "An Eddy-Permitting Southern Ocean State Estimate". In: Journal of Physical Oceanography 40.5, pp. 880899. ISSN: 0022-3670. DOI: 10.1175/2009JP04236.1.

McGillicuddy, D. J., L. A. Anderson, S. C. Doney, and M. E. Maltrud (2003). "Eddy-Driven Sources and Sinks of Nutrients in the Upper Ocean: Results from a $0.1^{\circ}$ Resolution Model of the North Atlantic". en. In: Global Biogeochemical Cycles 17.2. ISSN: 1944-9224. DOI: 10.1029/2002GB001987.

McGillicuddy, Dennis J. (2016). "Mechanisms of Physical-Biological-Biogeochemical Interaction at the Oceanic Mesoscale". In: Annual Review of Marine Science 8.1, pp. 125-159. DOI: $10.1146 /$ annurev-marine-010814-015606.

McGillicuddy, Dennis J., James R. Ledwell, and Laurence A. Anderson (2008). "Response to Comment on "Eddy/Wind Interactions Stimulate Extraordinary Mid-Ocean Plankton Blooms"'. en. In: Science 320.5875, pp. 448-448. ISSN: 0036-8075, 1095-9203. DOI: 10 . 1126/science. 1148974 .

McGillicuddy, Dennis J. et al. (1998). "Influence of Mesoscale Eddies on New Production in the Sargasso Sea". En. In: Nature 394.6690, p. 263. ISSN: 1476-4687. DOI: 10.1038/28367.

McGillicuddy, Dennis J. et al. (2007). "Eddy/Wind Interactions Stimulate Extraordinary MidOcean Plankton Blooms". eng. In: Science (New York, N. Y.) 316.5827, pp. 1021-1026. ISSN: 1095-9203. DOI: $10.1126 /$ science.1136256.

Meredith, Michael P. (2016). "Understanding the Structure of Changes in the Southern Ocean Eddy Field". In: Geophysical Research Letters 43.11, pp. 5829-5832. ISSN: 0094-8276. DOI: 10.1002/2016GL069677.

Michaels, Anthony F. and Mary W. Silver (1988). "Primary Production, Sinking Fluxes and the Microbial Food Web". In: Deep Sea Research Part A. Oceanographic Research Papers 35.4, pp. 473-490. ISSN: 0198-0149. DOI: 10.1016/0198-0149(88)90126-4.

Milutinović, Svetlana, Michael J. Behrenfeld, Johnny A. Johannessen, and Truls Johannessen (2009). "Sensitivity of Remote Sensing-Derived Phytoplankton Productivity to Mixed 
Layer Depth: Lessons from the Carbon-Based Productivity Model". en. In: Global Biogeochemical Cycles 23.4, GB4005. ISSN: 1944-9224. DOI: 10.1029/2008GB003431.

Misumi, Kazuhiro et al. (2014). "The Iron Budget in Ocean Surface Waters in the 20th and 21st Centuries : Projections by the Community Earth System Model Version 1". en. In: DOI: $10.5194 / \mathrm{bg}-11-33-2014$.

Monahan, Adam Hugh (2006). "The Probability Distribution of Sea Surface Wind Speeds. Part I: Theory and SeaWinds Observations". In: Journal of Climate 19.4, pp. 497-520. ISSN: 0894-8755. DOI: 10.1175/JCLI3640.1.

Montes-Hugo, Martin et al. (2009). "Recent Changes in Phytoplankton Communities Associated with Rapid Regional Climate Change along the Western Antarctic Peninsula". eng. In: Science (New York, N.Y.) 323.5920, pp. 1470-1473. ISSN: 1095-9203. DOI: 10.1126/ science.1164533.

Moore, C. Mark et al. (2007). "Iron-Light Interactions during the CROZet Natural Iron Bloom and EXport Experiment (CROZEX) I: Phytoplankton Growth and Photophysiology". In: Deep Sea Research Part II: Topical Studies in Oceanography. The Crozet Natural Iron Bloom and Export Experiment 54.18, pp. 2045-2065. ISSN: 0967-0645. DOI: $10.1016 / \mathrm{j}$. dsr2.2007.06.011.

Moore, J. Keith and Mark R. Abbott (2000). "Phytoplankton Chlorophyll Distributions and Primary Production in the Southern Ocean". en. In: Journal of Geophysical Research: Oceans 105.C12, pp. 28709-28722. ISSN: 2156-2202. DOI: 10.1029/1999JC000043.

Moore, J. Keith and Mark R Abbott (2002). "Surface Chlorophyll Concentrations in Relation to the Antarctic Polar Front: Seasonal and Spatial Patterns from Satellite Observations". In: Journal of Marine Systems. Physics and Biology of Ocean Fronts 37.1, pp. 69-86. ISSN: 0924-7963. DOI: 10.1016/S0924-7963(02)00196-3.

Moore, J. Keith and O. Braucher (2008). "Sedimentary and Mineral Dust Sources of Dissolved Iron to the World Ocean". In: Biogeosciences 5.3, pp. 631-656. ISSN: 1726-4189. DOI: 10. $5194 / \mathrm{bg}-5-631-2008$.

Moore, J. Keith, Scott C Doney, David M Glover, and Inez Y Fung (2001). "Iron Cycling and Nutrient-Limitation Patterns in Surface Waters of the World Ocean". In: Deep Sea Research Part II: Topical Studies in Oceanography. The US JGOFS Synthesis and Modeling Project: Phase 1 49.1, pp. 463-507. ISSN: 0967-0645. DOI: 10.1016/S0967-0645(01)00109-6.

Moore, J. Keith, Scott C. Doney, and Keith Lindsay (2004). "Upper Ocean Ecosystem Dynamics and Iron Cycling in a Global Three-Dimensional Model". en. In: Global Biogeochemical Cycles 18.4, GB4028. ISSN: 1944-9224. DOI: 10.1029/2004GB002220.

Moore, J. Keith, Keith Lindsay, Scott C. Doney, Matthew C. Long, and Kazuhiro Misumi (2013a). "Marine Ecosystem Dynamics and Biogeochemical Cycling in the Community Earth System Model [CESM1(BGC)]: Comparison of the 1990s with the 2090s under the 210 
RCP4.5 and RCP8.5 Scenarios". In: Journal of Climate 26.23, pp. 9291-9312. ISSN: 08948755. DOI: $10.1175 /$ JCLI-D-12-00566.1.

Moore, Mark C. et al. (2013b). "Processes and Patterns of Oceanic Nutrient Limitation". En. In: Nature Geoscience 6.9, p. 701. ISSN: 1752-0908. DOI: 10.1038/ngeo1765.

Morel, André and Jean-Francois Berthon (1989). "Surface Pigments, Algal Biomass Profiles, and Potential Production of the Euphotic Layer: Relationships Reinvestigated in View of Remote-Sensing Applications". en. In: Limnology and Oceanography 34.8, pp. 1545-1562. ISSN: 1939-5590. DOI: 10.4319/10.1989.34.8.1545.

Morrison, Adele K., Thomas L. Frölicher, and Jorge L. Sarmiento (2014). "Upwelling in the Southern Ocean". In: Physics Today 68.1, pp. 27-32. ISSN: 0031-9228. DOI: 10.1063/PT . 3.2654.

Nagao, Ippei et al. (2009). "Responses of DMS in the Seawater and Atmosphere to Iron Enrichment in the Subarctic Western North Pacific (SEEDS-II)". In: Deep Sea Research Part II: Topical Studies in Oceanography. SEEDS II: The Second Subarctic Pacific Iron Experiment for Ecosystem Dynamics Study 56.26, pp. 2899-2917. ISSN: 0967-0645. DOI: $10.1016 / j$.dsr2.2009.07.001.

Neeff, Till (2007). Market and Methodologies Meet Ocean Fertilization. en. Woods Hole, MA. USA.

Nelson, David M. and Walker 0. Smith (1991). "Sverdrup Revisited: Critical Depths, Maximum Chlorophyll Levels, and the Control of Southern Ocean Productivity by the IrradianceMixing Regime". en. In: Limnology and Oceanography 36.8, pp. 1650-1661. ISSN: 1939-5590. DOI: $10.4319 / 10.1991 .36 .8 .1650$.

"New Plankton-Seeding Venture Reaps \$3.5 Million" (2008). en. In: The New York Times.

Okubo, Akira (1970). "Horizontal Dispersion of Floatable Particles in the Vicinity of Velocity Singularities Such as Convergences". In: Deep Sea Research and Oceanographic Abstracts 17.3, pp. 445-454. ISSN: 0011-7471. DOI: 10.1016/0011-7471(70)90059-8.

O’Malley, Robert (2015). Ocean Productivity. science.oregonstate.edu/ocean.productivity.

O’Neill, Larry W., Dudley B. Chelton, and Steven K. Esbensen (2010). "The Effects of SSTInduced Surface Wind Speed and Direction Gradients on Midlatitude Surface Vorticity and Divergence". In: Journal of Climate 23.2, pp. 255-281. ISSN: 0894-8755. DOI: 10.1175/ 2009JCLI2613. 1.

Oschlies, Andreas, Woldgang Koeve, Wilfried Rickels, and Katrin Rehdanz (2010). "Side Effects and Accounting Aspects of Hypothetical Large-Scale Southern Ocean Iron Fertilization". In: Biogeosciences 7.12, pp. 4017-4035. ISSN: 1726-4189. DOI: 10.5194/bg-7-40172010. 
Pacala, Stephen and Robert Socolow (2004). "Stabilization Wedges: Solving the Climate Problem for the Next 50 Years with Current Technologies". en. In: Science 305.5686, pp. 968972. ISSN: 0036-8075, 1095-9203. DOI: 10.1126/science.1100103.

Park, Jisoo et al. (2017). "Light Availability Rather than Fe Controls the Magnitude of Massive Phytoplankton Bloom in the Amundsen Sea Polynyas, Antarctica". en. In: Limnology and Oceanography 62.5, pp. 2260-2276. ISSN: 1939-5590. DOI: 10.1002/lno.10565.

Pegau, W. Scott, Emmanuel Boss, and Antonio Martínez (2002). "Ocean Color Observations of Eddies during the Summer in the Gulf of California". en. In: Geophysical Research Letters 29.9. ISSN: 1944-8007. DOI: 10.1029/2001GL014076.

Pollard, Raymond T. et al. (2009). "Southern Ocean Deep-Water Carbon Export Enhanced by Natural Iron Fertilization". English. In: Nature 457.7229, pp. 577-580. ISSN: 0028-0836. DOI: $10.1038 /$ nature07716.

Prants, S. V., M. V. Budyansky, V. I. Ponomarev, and M. Yu. Uleysky (2011). "Lagrangian Study of Transport and Mixing in a Mesoscale Eddy Street". In: Ocean Modelling 38.1, pp. 114-125. ISSN: 1463-5003. DOI: 10.1016/j.ocemod.2011.02.008.

Prentice, I. Colin (2001). IPCC: Climate Change 2001: The Physical Science Basis. Contribution of Working Group I to the Third Assessment Report of the Intergovernmental Panel on Climate 3.2.3 Ocean Carbon Processes. Tech. rep., p. 197.

Pujol, Marie-Isabelle et al. (2016). "DUACS DT2014: The New Multi-Mission Altimeter Data Set Reprocessed over 20 Years". en. In: Ocean Science 12.5, pp. 1067-1090. ISSN: 1812-0792. DOI: $10.5194 /$ os-12-1067-2016.

Quéré, Corinne Le, Laurent Bopp, and Ina Tegen (2002). "Antarctic Circumpolar Wave Impact on Marine Biology: A Natural Laboratory for Climate Change Study". en. In: Geophysical Research Letters 29.10, pp. 45-1-45-4. ISSN: 1944-8007. DOI: 10.1029/2001GL014585.

Quéré, Corinne Le et al. (2016). "Role of Zooplankton Dynamics for Southern Ocean Phytoplankton Biomass and Global Biogeochemical Cycles". English. In: Biogeosciences 13.14, pp. 4111-4133. ISSN: 1726-4170. DOI: https://doi.org/10.5194/bg-13-4111-2016.

Quinn, Patricia K. and Timothy S. Bates (2011). "The Case against Climate Regulation via Oceanic Phytoplankton Sulphur Emissions". eng. In: Nature 480.7375, pp. 51-56. ISSN: 1476-4687. DOI: 10.1038/nature10580.

Racault, Marie-Fanny, Corinne Le Quéré, Erik Buitenhuis, Shubha Sathyendranath, and Trevor Platt (2012). "Phytoplankton Phenology in the Global Ocean". In: Ecological Indicators 14, pp. 152-163. DOI: $10.1016 / \mathrm{j}$.ecolind.2011.07.010.

Ramaswamy, Venkatachalam et al. (2001). "Radiative Forcing of Climate Change". en. In: Climate Change 2001: The Scientific Basis. Vol. 18. Cambridge, United Kingdom: Cambridge University Press, pp. 349-416. 
Reichle, Dave et al. (1999). WORKING PAPER ON CARBON SEQUESTRATION SCIENCE AND TECHNOLOGY. en. Tech. rep. Office of Science, Office of Fossil Energy, U.S. Department of Energy, p. 223.

LC-LP.1 (2008). Resolution LC-LP.1 on the Regulation of Ocean Iron Ferilization. London Convention and Protocol. Tech. rep.

LC-LP.4 (2013). Resolution LP.4 (8) on the Amendment to the London Protocol to Regulate the Placement of Matter for Ocean Fertilization and Other Marine Geoengineering Activities, LP.8, LC 35/15, Annex 4, Annex 5. Tech. rep.

Riebeek, Holli (2011). The Carbon Cycle : Feature Articles. www.earthobservatory.nasa.gov.

Riley, Gordon (1946). Factors Controlling Phytoplankton Populations on Georges Bank. en.

Rintoul, Stephen R., Chris W. Hughes, and Dirk Olbers (2001). "Chapter 4.6 The Antarctic Circumpolar Current System". In: International Geophysics. Ed. by Gerold Siedler, John Church, and John Gould. Vol. 77. Ocean Circulation and Climate. Academic Press, pp. 271XXXVI. DOI: 10.1016/S0074-6142(01)80124-8.

Robock, Alan, Kirsten Jerch, and Martin Bunzl (2008). "20 Reasons Why Geoengineering May Be a Bad Idea". In: Bulletin of the Atomic Scientists 64.2, pp. 14-59. ISSN: 0096-3402. DOI: 10.1080/00963402.2008.11461140.

Rohr, Tyler, Matthew Long, Maria T. Kavanaugh, Keith Lindsay, and Scott Doney (2017). "Variability in the Mechanisms Controlling Southern Ocean Phytoplankton Bloom Phenology in an Ocean Model and Satellite Observations". In: Global Biogeochemical Cycles 31. DOI: $10.1002 / 2016$ gb005615.

Ruddick, Kevin, Griet Neukermans, Quinten Vanhellemont, and Dominique Jolivet (2014). "Challenges and Opportunities for Geostationary Ocean Colour Remote Sensing of Regional Seas: A Review of Recent Results". In: Remote Sensing of Environment. Liege Colloquium Special Issue: Remote sensing of ocean colour, temperature and salinity 146, pp. 63-76. ISSN: 0034-4257. DOI: 10.1016/j.rse.2013.07.039.

Saba, Vincent S. et al. (2011). "An Evaluation of Ocean Color Model Estimates of Marine Primary Productivity in Coastal and Pelagic Regions across the Globe". English. In: Biogeosciences 8.2, pp. 489-503. ISSN: 1726-4170. DOI: https ://doi .org/10.5194/bg-8489-2011.

Sakshaug, Egil and Osmund Holm-Hansen (1986). "Photoadaptation in Antarctic Phytopfankton: Variations in Growth Rate, Chemical Composition and P versus I Curves". en. In: Journal of Plankton Research 8.3, pp. 459-473. ISSN: 0142-7873. DOI: 10.1093/plankt/8. 3.459 .

Sallée, J.-B., E. Shuckburgh, N. Bruneau, A. J. S. Meijers, T. J. Bracegirdle, and Z. Wang (2013). "Assessment of Southern Ocean Mixed-Layer Depths in CMIP5 Models: Histori- 
cal Bias and Forcing Response". en. In: Journal of Geophysical Research: Oceans 118.4, pp. 1845-1862. ISSN: 2169-9291. DOI: 10.1002/jgrc. 20157.

Salter, Ian, Alan E. S. Kemp, C. Mark Moore, Richard S. Lampitt, George A. Wolff, and Jens Holtvoeth (2012). "Diatom Resting Spore Ecology Drives Enhanced Carbon Export from a Naturally Iron-Fertilized Bloom in the Southern Ocean". en. In: Global Biogeochemical Cycles 26.1. ISSN: 1944-9224. DOI: 10.1029/2010GB003977.

Salter, Ian, Ralf Schiebel, Patrizia Ziveri, Aurore Movellan, Richard Lampitt, and George A. Wolff (2014). "Carbonate Counter Pump Stimulated by Natural Iron Fertilization in the Polar Frontal Zone". en. In: Nature Geoscience 7.12, pp. 885-889. ISSN: 1752-0908. DOI: $10.1038 /$ ngeo2285.

Samelson, R.m. (2013). "Lagrangian Motion, Coherent Structures, and Lines of Persistent Material Strain". In: Annual Review of Marine Science 5.1, pp. 137-163. ISSN: 1941-1405. DOI: $10.1146 /$ annurev-marine-120710-100819.

Sarmiento, Jorge L. and James C. Orr (1991). "Three-Dimensional Simulations of the Impact of Southern Ocean Nutrient Depletion on Atmospheric CO2 and Ocean Chemistry". en. In: Limnology and Oceanography 36.8, pp. 1928-1950. ISSN: 1939-5590. DOI: 10.4319/1o. 1991.36.8.1928.

Sarmiento, Jorge L. et al. (2004). "Response of Ocean Ecosystems to Climate Warming". en. In: Global Biogeochemical Cycles 18.3. ISSN: 1944-9224. DOI: 10.1029/2003GB002134.

Schoemann, Véronique, Sylvie Becquevort, Jacqueline Stefels, Véronique Rousseau, and Christiane Lancelot (2005). "Phaeocystis Blooms in the Global Ocean and Their Controlling Mechanisms: A Review". In: Journal of Sea Research. Iron Resources and Oceanic Nutrients - Advancement of Global Environmental Simulations 53.1, pp. 43-66. ISSN: 1385-1101. DOI: $10.1016 / j$.seares.2004.01.008.

Sedwick, Peter N. and Giacomo R. DiTullio (1997). "Regulation of Algal Blooms in Antarctic Shelf Waters by the Release of Iron from Melting Sea Ice". en. In: Geophysical Research Letters 24.20, pp. 2515-2518. ISSN: 1944-8007. DOI: 10.1029/97GL02596.

Shang, Shaoling L. et al. (2010). "Comparison of Primary Productivity Models in the Southern Ocean: Preliminary Results". In: Ocean Sensing and Monitoring II. Vol. 7678, p. 767808. DOI: $10.1117 / 12.853631$.

Shear, Michael D. (2018). "Trump Will Withdraw U.S. From Paris Climate Agreement". en. In: The New York Times. ISSN: 0362-4331.

Siegel, David A., Ken O. Buesseler, Scott C. Doney, Sarah F. Sailley, Michael J. Behrenfeld, and Philip W. Boyd (2014). "Global Assessment of Ocean Carbon Export by Combining Satellite Observations and Food-Web Models". en. In: Global Biogeochemical Cycles 28.3, 2013GB004743. ISSN: 1944-9224. DOI: 10.1002/2013GB004743. 
Siegel, David A., David B. Court, David W. Menzies, Pete Peterson, Stephane Maritorena, and Norman B. Nelson (2008). "Satellite and in Situ Observations of the Bio-Optical Signatures of Two Mesoscale Eddies in the Sargasso Sea". In: Deep Sea Research Part II: Topical Studies in Oceanography. Mesoscale Physical-Biological-Biogeochemical Linkages in the Open Ocean: Results from the E-FLUX and EDDIES Programs 55.10, pp. 12181230. ISSN: 0967-0645. DOI: 10.1016/j.dsr2.2008.01.012.

Siegel, David A., Scott C. Doney, and James A. Yoder (2002). "The North Atlantic Spring Phytoplankton Bloom and Sverdrup's Critical Depth Hypothesis". en. In: Science 296.5568, pp. 730-733. ISSN: 0036-8075, 1095-9203. DOI: 10.1126/science.1069174.

Siegel, David A., Dennis J. McGillicuddy, and Erik A. Fields (1999). "Mesoscale Eddies, Satellite Altimetry, and New Production in the Sargasso Sea". en. In: Journal of Geophysical Research: Oceans 104.C6, pp. 13359-13379. ISSN: 2156-2202. DOI: 10.1029/1999JC900051.

Siegel, David A., Pete Peterson, Dennis J. McGillicuddy, Stephane Maritorena, and Norman B. Nelson (2011). "Bio-Optical Footprints Created by Mesoscale Eddies in the Sargasso Sea". en. In: Geophysical Research Letters 38.13, p. L13608. ISSN: 1944-8007. DOI: 10.1029/ 2011 GL047660.

Sigman, Daniel M. and Edward A. Boyle (2000). "Glacial/Interglacial Variations in Atmospheric Carbon Dioxide". eng. In: Nature 407.6806, pp. 859-869. ISSN: 0028-0836. DOI: $10.1038 / 35038000$.

Silver, Mary W. et al. (2010). "Toxic Diatoms and Domoic Acid in Natural and Iron Enriched Waters of the Oceanic Pacific". en. In: Proceedings of the National Academy of Sciences 107.48, pp. 20762-20767. ISSN: 0027-8424, 1091-6490. DOI: 10.1073/pnas. 1006968107.

Smetacek, Victor et al. (2012). "Deep Carbon Export from a Southern Ocean Iron-Fertilized Diatom Bloom". eng. In: Nature 487.7407, pp. 313-319. ISSN: 1476-4687. DOI: $10.1038 /$ nature11229.

Smith, R. et al. (2010). "The Parallel Ocean Program (POP) Reference Manual: Ocean Component of the Community Climate System Model (CCSM)". en. In:

Smith, Walker O. and Josefino C. Comiso (2008). "Influence of Sea Ice on Primary Production in the Southern Ocean: A Satellite Perspective". en. In: Journal of Geophysical Research: Oceans 113.C5, C05S93. ISSN: 2156-2202. DOI: 10.1029/2007JC004251.

Smith, Walker O. and David M. Nelson (1985). "Phytoplankton Bloom Produced by a Receding Ice Edge in the Ross Sea: Spatial Coherence with the Density Field". eng. In: Science (New York, N.Y.) 227.4683, pp. 163-166. ISSN: 0036-8075. DOI: 10.1126/science.227.4683. 163.

Smith, Walker O., David M. Nelson, and Sylvie Mathot (1999). "Phytoplankton Growth Rates in the Ross Sea, Antarctica, Determined by Independent Methods: Temporal Variations". 
In: Journal of Plankton Research 21.8, pp. 1519-1536. ISSN: 0142-7873. DOI: 10 . $1093 /$ $\mathrm{plankt/21.8.1519.}$

Song, Hajoon, Matthew C. Long, Peter Gaube, Ivy Frenger, John Marshall, and Dennis J. McGillicuddy (2018). "Seasonal Variation in the Correlation Between Anomalies of Sea Level and Chlorophyll in the Antarctic Circumpolar Current". en. In: Geophysical Research Letters 45.10, pp. 5011-5019. ISSN: 1944-8007. DOI: 10.1029/2017GL076246.

Spies, Annette (1987). "Growth Rates of Antarctic Marine Phytoplankton in the Weddell Sea". In: Marine Ecology Progress Series 41.3, pp. 267-274. ISSN: 0171-8630.

Stammerjohn, Sharon, Robert Massom, David Rind, and Douglas Martinson (2012). "Regions of Rapid Sea Ice Change: An Inter-Hemispheric Seasonal Comparison". en. In: Geophysical Research Letters 39.6, p. L06501. ISSN: 1944-8007. DOI: 10.1029/2012GL050874.

Stammerjohn, Sharron E., Doug G. Martinson, Ray C. Smith, Xiaojun Yuan, and David Rind (2008). "Trends in Antarctic Annual Sea Ice Retreat and Advance and Their Relation to El Niño-Southern Oscillation and Southern Annular Mode Variability". en. In: Journal of Geophysical Research: Oceans 113.C3, C03S90. ISSN: 2156-2202. DOI: 10.1029/2007 JC004269.

Stevens, David P. and Peter D. Killworth (1992). "The Distribution of Kinetic Energy in the Southern Ocean: A Comparison between Observations and an Eddy Resolving General Circulation Model". en. In: Phil. Trans. R. Soc. Lond. B 338.1285, pp. 251-257. ISSN: 0962-8436, 1471-2970. DOI: 10.1098/rstb.1992.0145.

Stock, Charles A. et al. (2017). "Reconciling Fisheries Catch and Ocean Productivity". eng. In: 114.8, E1441-E1449. ISSN: 1091-6490. DOI: 10.1073/pnas.1610238114.

Stroeve, Julienne C., Stéphanie Jenouvrier, G. Garrett Campbell, Christophe Barbraud, and Delord Karine (2016). "Mapping and Assessing Variability in the Antarctic Marginal Ice Zone, Pack Ice and Coastal Polynyas in Two Sea Ice Algorithms with Implications on Breeding Success of Snow Petrels". In: The Cryosphere 10.4, pp. 1823-1843. ISSN: 19940424. DOI: $10.5194 /$ tc-10-1823-2016.

Sullivan, Cornelius W., Kevin R. Arrigo, Charles R. McClain, Josefino C. Comiso, and James Firestone (1993). "Distributions of Phytoplankton Blooms in the Southern Ocean". eng. In: Science (New York, N.Y.) 262.5141, pp. 1832-1837. ISSN: 0036-8075. DOI: 10.1126/ science.262.5141.1832.

Sunda, William G. and Susan A. Huntsman (1995). "Iron Uptake and Growth Limitation in Oceanic and Coastal Phytoplankton". en. In: Marine Chemistry 50.1-4, pp. 189-206. ISSN: 03044203. DOI: 10.1016/0304-4203(95)00035-P.

Sverdrup, Harald U. (1953). "On Conditions for the Vernal Blooming of Phytoplankton". en. In: ICES Journal of Marine Science 18.3, pp. 287-295. ISSN: 1054-3139. DOI: 10.1093/ icesjms/18.3.287. 
Tagliabue, Alessandro, Laurent Bopp, and Olivier Aumont (2009). "Evaluating the Importance of Atmospheric and Sedimentary Iron Sources to Southern Ocean Biogeochemistry". en. In: Geophysical Research Letters 36.13, p. L13601. ISSN: 1944-8007. DOI: 10 . 1029 / 2009 GL038914.

Tagliabue, Alessandro, Jean-Baptiste Sallée, Andrew R. Bowie, Marina Lévy, Sebastiaan Swart, and Philip W. Boyd (2014). "Surface-Water Iron Supplies in the Southern Ocean Sustained by Deep Winter Mixing". En. In: Nature Geoscience 7.4, p. 314. ISSN: 1752-0908. DOI: $10.1038 /$ ngeo2101.

Takeda, Shigenobu and Atsushi Tsuda (2005). "An in Situ Iron-Enrichment Experiment in the Western Subarctic Pacific (SEEDS): Introduction and Summary". In: Progress in Oceanography 64, pp. 95-109. ISSN: 0079-6611. DOI: 10.1016/j · pocean.2005.02.004.

Tamsitt, Veronica et al. (2017). "Spiraling Pathways of Global Deep Waters to the Surface of the Southern Ocean". En. In: Nature Communications 8.1, p. 172. ISSN: 2041-1723. DOI: 10.1038/s41467-017-00197-0.

Tang, Kam W., Walker O. Smith, Amy R. Shields, and David T. Elliott (2009). "Survival and Recovery of Phaeocystis Antarctica (Prymnesiophyceae) from Prolonged Darkness and Freezing". en. In: Proceedings of the Royal Society of London B: Biological Sciences 276.1654, pp. 81-90. ISSN: 0962-8452, 1471-2954. DOI: 10.1098/rspb.2008.0598.

Taylor, John R. and Raffaele Ferrari (2011). "Shutdown of Turbulent Convection as a New Criterion for the Onset of Spring Phytoplankton Blooms". en. In: Limnology and Oceanography 56.6, pp. 2293-2307. ISSN: 1939-5590. DOI: 10.4319/10.2011.56.6.2293.

Thomalla, S. J., N. Fauchereau, S. Swart, and P. M. S. Monteiro (2011). "Regional Scale Characteristics of the Seasonal Cycle of Chlorophyll in the Southern Ocean". In: Biogeosciences 8.10, pp. 2849-2866. ISSN: 1726-4189. DOI: 10.5194/bg-8-2849-2011.

Thompson, Alexander (2006). "Management Under Anarchy: The International Politics of Climate Change". en. In: Climatic Change 78.1, pp. 7-29. ISSN: 0165-0009, 1573-1480. DOI: 10.1007/s10584-006-9090-x.

Tollefson, Jeff (2017). "Iron-Dumping Ocean Experiment Sparks Controversy". en. In: Scientific American.

Townsend, David W., Maureen D. Keller, Michael E. Sieracki, and Steven G. Ackleson (1992). "Spring Phytoplankton Blooms in the Absence of Vertical Water Column Stratification". En. In: Nature 360.6399, p. 59. ISSN: 1476-4687. DOI: 10.1038/360059a0.

Tréguer, Paul and Philippe Pondaven (2002). "Preface: Climatic Changes and the Carbon Cycles in the Southern Ocean: A Step Forward". In: Deep Sea Research Part II Topical Studies in Oceanography 49, pp. 1597-1600. DOI: 10.1016/S0967-0645(02)00002-4.

Trick, Charles G., Brian D. Bill, William P. Cochlan, Mark L. Wells, Vera L. Trainer, and Lisa D. Pickell (2010). "Iron Enrichment Stimulates Toxic Diatom Production in High-Nitrate, 
Low-Chlorophyll Areas". en. In: Proceedings of the National Academy of Sciences 107.13, pp. 5887-5892. ISSN: 0027-8424, 1091-6490. DOI: 10.1073/pnas.0910579107.

Tsuda, Atsushi, Hiroaki Saito, Jun Nishioka, Tsuneo Ono, Yoshifumi Noiri, and Isao Kudo (2006). "Mesozooplankton Response to Iron Enrichment during the Diatom Bloom and Bloom Decline in SERIES (NE Pacific)". In: Deep Sea Research Part II Topical Studies in Oceanography 53.20-22, pp. 2281-2296. ISSN: 0967-0645. DOI: 10.1016/j.dsr2.2006.05. 041.

Tsuda, Atsushi, Hiroaki Saito, Shigenobu Takeda, and Naoki Yoshie (2007). "Evidence for the Grazing Hypothesis: Grazing Reduces Phytoplankton Responses of the HNLC Ecosystem to Iron Enrichment in the Western Subarctic Pacific (SEEDS II)". en. In: Journal of Oceanography 63(6), pp. 983-994. DOI: 10.1007/s10872-007-0082-x.

"Antarctic Climate Change and the Environment" (2010). In: Chemistry International - Newsmagazine for IUPAC 32.2. Ed. by J Turner and R.A. Bindschadler, pp. 25-26. ISSN: 13652192. DOI: $10.1515 / c i .2010 .32 .2 .25$.

Turner, Suzanne M., M. J. Harvey, Cliff S. Law, Philip D. Nightingale, and Peter S. Liss (2004). "Iron-Induced Changes in Oceanic Sulfur Biogeochemistry". en. In: Geophysical Research Letters 31.14. ISSN: 1944-8007. DOI: 10.1029/2004GL020296.

Turner, Suzanne M., Philip D. Nightingale, Lucinda J. Spokes, Malcolm I. Liddicoat, and Peter S. Liss (1996). "Increased Dimethyl Sulphide Concentrations in Sea Water from in Situ Iron Enrichment". en. In: Nature 383.6600, pp. 513-517. ISSN: 1476-4687. DOI: 10.1038/383513a0.

Uitz, Julia, Hervé Claustre, André Morel, and Stanford B. Hooker (2006). "Vertical Distribution of Phytoplankton Communities in Open Ocean: An Assessment Based on Surface Chlorophyll". en. In: Journal of Geophysical Research: Oceans 111.C8, p. C08005. ISSN: 2156-2202. DOI: 10.1029/2005JC003207.

van der Ploeg, Rienk R., W. Bohm, and M. B. Kirkham (1999). "On the Origin of the Theory of Mineral Nutrition of Plants and the Law of the Minimum". en. In: 63.5, pp. 1055-1062. ISSN: 1435-0661. DOI: 10.2136/sssaj1999.6351055x.

Varpe, Øystein (2012). "Fitness and Phenology: Annual Routines and Zooplankton Adaptations to Seasonal Cycles". en. In: Journal of Plankton Research 34.4, pp. 267-276. ISSN: 0142-7873. DOI: $10.1093 / \mathrm{plankt/fbr108.}$

Venables, Hugh J. and Michael P. Meredith (2009). "Theory and Observations of Ekman Flux in the Chlorophyll Distribution Downstream of South Georgia". en. In: Geophysical Research Letters 36.23. ISSN: 1944-8007. DOI: 10.1029/2009GL041371.

Walter, Sylvia, Ilka Peeken, Karin Lochte, Adrian Webb, and Hermann W. Bange (2005). "Nitrous Oxide Measurements during EIFEX, the European Iron Fertilization Experiment 
in the Subpolar South Atlantic Ocean". en. In: Geophysical Research Letters 32.23. ISSN: 1944-8007. DOI: 10.1029/2005GL024619.

Wang, Shanlin, D. Bailey, Keith Lindsay, J. Keith Moore, and M. Holland (2014). "Impact of Sea Ice on the Marine Iron Cycle and Phytoplankton Productivity". In: Biogeosciences 11.17, pp. 4713-4731. ISSN: 1726-4189. DOI: 10.5194/bg-11-4713-2014.

Wang, Shanlin and J. Keith Moore (2011). "Incorporating Phaeocystis into a Southern Ocean Ecosystem Model". en. In: Journal of Geophysical Research: Oceans 116.C1, p. C01019. ISSN: 2156-2202. DOI: 10.1029/2009JC005817.

Watson, Andrew J., Philip W. Boyd, Suzanne M. Turner, Timothy D. Jickells, and Peter S. Liss (2008). "Designing the next Generation of Ocean Iron Fertilization Experiments". en. In: Marine Ecology Progress Series 364, pp. 303-309. ISSN: 0171-8630, 1616-1599. DOI: $10.3354 /$ meps 07552 .

Watson, Reg, Dirk Zeller, and Daniel Pauly (2013). "Primary Production Demands of Global Fisheries". In: Fish and Fisheries 15. DOI: 10.1111/faf.12013.

Webb, Douglas C., Paul J. Simonetti, and Clayton P. Jones (2001). "SLOCUM: An Underwater Glider Propelled by Environmental Energy". In: IEEE Journal of Oceanic Engineering 26.4, pp. 447-452. ISSN: 0364-9059. DOI: 10.1109/48.972077.

Weijer, Wilbert et al. (2011). "The Southern Ocean and Its Climate in CCSM4". In: Journal of Climate 25.8, pp. 2652-2675. ISSN: 0894-8755. DOI: 10.1175/JCLI-D-11-00302.1.

Weiss, John (1991). "The Dynamics of Enstrophy Transfer in Two-Dimensional Hydrodynamics". In: Physica D: Nonlinear Phenomena 48.2, pp. 273-294. ISSN: 0167-2789. DOI: 10.1016/0167-2789 (91)90088-Q.

Westberry, Toby K., Michael J. Behrenfeld, David A. Siegel, and Emanuel S. Boss (2008). "Carbon-Based Primary Productivity Modeling with Vertically Resolved Photoacclimation". en. In: Global Biogeochemical Cycles 22.2, GB2024. ISSN: 1944-9224. DOI: 10.1029/ 2007 GB003078.

Williams, Sean et al. (2011). "Adaptive Extraction and Quantification of Geophysical Vortices". In: IEEE Transactions on Visualization and Computer Graphics 17.12, pp. 2088-2095. ISSN: 1077-2626. DOI: 10.1109/TVCG.2011.162.

Winckler, Gisela, Robert F. Anderson, Martin Q. Fleisher, David McGee, and Natalie Mahowald (2008). "Covariant Glacial-Interglacial Dust Fluxes in the Equatorial Pacific and Antarctica". en. In: Science 320.5872, pp. 93-96. ISSN: 0036-8075, 1095-9203. DOI: 10 . 1126/science.1150595.

Winckler, Gisela, Robert F. Anderson, Samuel L. Jaccard, and Franco Marcantonio (2016). "Ocean Dynamics, Not Dust, Have Controlled Equatorial Pacific Productivity over the Past 500,000 Years". en. In: Proceedings of the National Academy of Sciences 113.22, pp. 61196124. ISSN: 0027-8424, 1091-6490. DOI: 10.1073/pnas.1600616113. 
Worstall, Tim (2012). "The Cheap Way To Deal With Climate Change: Iron Fertilisation Of The Oceans". en. In: Forbes.

Xavier, José C. et al. (2016). "Future Challenges in Southern Ocean Ecology Research". English. In: Frontiers in Marine Science 3. ISSN: 2296-7745. DOI: 10.3389 /fmars . 2016 . 00094.

Xiu, Peng, Artur P. Palacz, Fei Chai, Eric G. Roy, and Mark L. Wells (2011). "Iron Flux Induced by Haida Eddies in the Gulf of Alaska". en. In: Geophysical Research Letters 38.13. ISSN: 1944-8007. DOI: 10.1029/2011GL047946.

Yoon, Joo-Eun et al. (2016). "Ocean Iron Fertilization Experiments: Past-Present-Future with Introduction to Korean Iron Fertilization Experiment in the Southern Ocean (KIFES) Project". In: Biogeosciences Discuss. 2016, pp. 1-41. ISSN: 1810-6285. DOI: 10.5194/bg$2016-472$.

Zahariev, Konstantin, James R. Christian, and Kenneth L. Denman (2008). "Preindustrial, Historical, and Fertilization Simulations Using a Global Ocean Carbon Model with New Parameterizations of Iron Limitation, Calcification, and N 2 Fixation". In: Progress in Oceanography 77, pp. 56-82. ISSN: 0079-6611. DOI: 10.1016/j · pocean.2008.01.007.

Zhang, Shuwen, Enrique N. Curchitser, Dujuan Kang, Charles A. Stock, and Raphael Dussin (2018). "Impacts of Mesoscale Eddies on the Vertical Nitrate Flux in the Gulf Stream Region". In: Journal of Geophysical Research: Oceans. DOI: 10.1002/2017JC013402. 